Environmental Contaminants in Food

December 1979

NTIS order \#PB80-153265

\title{
Environmental Contaminants in Food
}




\section{Library of Congress Catalog Card Number 79-600207}

For sale by the Superintendent of Documents, U.S. Government Printing Office Washington, D.C. 20402 Stock No. 052-003-00724-0 


\section{FOREWORD}

This report presents the major findings of an OTA assessment of Federal and State efforts to deal with the environmental contamination of food. Undertaken at the request of the House Committee on Interstate and Foreign Commerce, the study examines both regulatory approaches and monitoring strategies for coping with contaminated food.

The assessment is concerned with chemical and radioactive contaminants that inadvertently find their way into the human food supply. To bring the scope of inquiry within manageable bounds, we excluded naturally occurring toxins such as fungal and microbial toxins.

The Office of Technology Assessment was assisted by two advisory panels of scientists and representatives of public interest groups, agriculture, the chemical industry, fisheries, and State and foreign governments. The Food and Drug Administration, the Department of Agriculture, and the Environmental Protection Agency each designated staff members to attend panel meetings, provide background information, and review draft reports, Background papers were commissioned concerning the scientific aspects of detecting and regulating environmental contaminants in food. The Congressional Research Service provided five analyses of previous food contamination episodes, Reviews of the draft report were provided by the advisory panels, Federal agencies, and a number of interested individuals not previously involved with the assessment.

Because this assessment addresses concerns of American citizens as well as policy makers and scientists, the summary of the report is also being published as a separate document. The summary provides the interested citizen with an informative and clear overview of this complex problem. Copies of the summary can he obtained free of charge from the office of Technology Assessment, U.S. Congress, Washington, D.C. 20510.

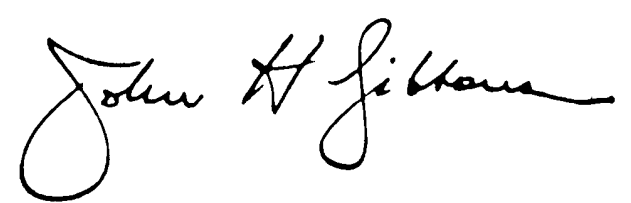

JOHN H. GIBBONS

Director 


\section{Tolerance Advisory Panel}

James Anderson

Manager for Material Safety

Allied Chemical Corporation

Louise Burke

Executive Director

Consumer Council of Virginia Foundation

Consumer Council of Virginia, Inc.

Kenneth Crump

Department of Mathematics and Statistics

Louisiana Tech University

Donald Epp

Department of Agricultural Economics and Rural Sociology

Pennsylvania Stale [University

Frederick Halbert

Dairy Farmer

Delton, Mich.

Joseph Highland

Chairman

Toxic Chemicals Program

Environmental Defense Fund

\section{Monitoring Advisory Panel}

Raymond Allred

Director

Environmental Conservation Research

Continental Oil Company

Richard Anderson

Chairman

Statistics Department

University of Kentucky

John Bourke

Director

Analytical Division

Food Science Department

Cornell University

Vaughan Bowen

Woods Hole Oceanographic Institute

Shirley Briggs

Executive Director

Rachel Carson Trust for the Living

Environment, Inc.

Leon Chesnin

Department of Agronomy

University of Nebraska
Robert Jackson

Assistant State Health Commissioner

Director, Office of Health Protection and Environmental Management

Virginia State Health Department

Kazuo K, Kimura

School of Medicine

Wright State University

Marvin Legator

Director

Division of Environmental Toxicology

Deportment of Preventive Medicine and Community Health

University of Texas Medical Branch

David Lindsay

Technical Secretary

Steering Group on Food Surveillance

Ministry of Agriculture, Fisheries, and Food

United Kingdom

Roy Mart in

Director

Science and Technology

National Fisheries Institute, Inc.
Richard Merrill

School of Law

University of Virginia

Joseph Rosen

Department of Food Science

Rutgers University

John Rust

Professor Emeritus

Pharmacology/Radiology

University of Chicago

Gary Van Gelder

College of Veterinary Medicine

University of Missouri

John Van Ryzin

RAND Corporation

George Whitehead

Deputy Director

Office for Consumer Affairs

Michigan Department of Agriculture
William Cooper

Professor

Department of Zoology

College of Natural Science

Michigan State University

John Davies

Chairman

Department of Epidemiology and Public Health

School of Medicine

Universitv of Miami

Blake Early

Environmental Action, Inc.

Edward Elkins

Director

Chemistry Division

Washington Research Laboratory

National Food Processors' Association

Ronald J. Hingle

Administrator

Food and Drug Control Unit

Bureau of Environmental Services

Louisiana Office of Health Services and Environmental Quality
S. Roy Koirtyohann

Environmental Trace Substances Center

University of Missouri

Charles Krister

Retired Manager

Biochemicals Department

E. I. DuPont de Nemours and Company

John Laseter

Director

Center for Bio-Organic Studies

University of New or leans

John Martin

Moss Landing Marine Laboratories

Rebecca Sharitz

Savannah River Ecology Laboratories

University of Georgia

Gerald Ward

Department of Animal Science

Colorado State University 


\section{Food and Renewable Resources Program Staff}

Joyce C. Lashof, Assistant Director

Health and Life Sciences Division

J. B. Cordaro, Program Manager*

Walter Parham, Program Manager

Catherine Woteki, Project Director

Robert Smith, Research Assistant

Chris Elf ring, OTA Congressional Fellow

Administrative Staff

Phyllis Balan Elizabeth Galloway Ogechee Koffler

\section{Contractors}

Lloyd W. Hazleton, Technical Advisor to Tolerance Panel

Robert Huggett, Technical Advisor to Monitoring Panel

Samuel Iker, Editor

\section{OTA Publishing Staff}

John C. Holmes, Publishing Officer

Kathie S. Boss Joanne Heming

\section{Food Advisory Committee}

\author{
Martin E. Abel, Chairmen \\ Senior Vice President \\ Schnittker Associates \\ Johanna Dwyer, Co-Chairman \\ Director \\ Frances Stern Nutrition Center \\ David Call \\ Dean, College of Agricultural \\ and Life Sciences \\ Cornell University \\ Cy Carpenter \\ President \\ Minnesota Farmers Union \\ Eliot Coleman \\ Director \\ Coolidge Center for the \\ Study of Agriculture \\ Almeta Edwards Fleming \\ Social Program Coordinator \\ Florence County, S.C.
}

Lorne Greene

Chairman of the Board

American Freedom From

Hunger Foundation

Richard L. Hall

Vice President, Science and Technology

McCormick \& Company, Inc.

Laura Heuser

Member, Board of Directors

Agricultural Council of America

Arnold Mayer

Legislative Representative

Amalgamated Meat Cutters and

Butcher Workmen of North

America

Max Milner

Executive Director

American Institute of Nutrition
Robert 0. Nesheim

Vice President, Science and Technology

The Quaker oats Company

Kathleen O'Reilly

Director

Consumer Federation of America

R. Dennis Rouse

Dean, School of Agriculture

Auburn University

Lauren Seth

Agricultural Consultant

Thomas Sporleder

Professor of Agricultural

Economics

Texas A\&M University

Sylvan Wittwer

Director and Assistant Dean

College of Agriculture and

Natural Resources

Michigan State University

\footnotetext{
*Resigned June 16,1979, and continued to serve under contract to assessment completion.
} 


\section{Contents}

I. Summary . . .

Health Impacts of Environmental Contamination. $\ldots \ldots \ldots \ldots \ldots \ldots \ldots$

Economic Impacts of Contamination. . . . . . . . . . . . . . . . . Major Problems in Identifying Environmental Contaminants . . . . . . . . . . . . . Problems of Regulating Environmental Contaminants. . . . . . . . . . . . Findings and Conclusions . . . . . . . . . ............... Congressional Options. . . . . . . . . . . . . . . . . . . . . . . . . . . .

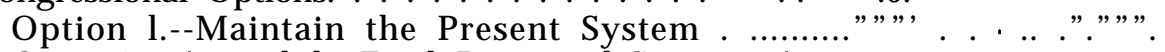
Option2.-Amend the Food, Drug, and Cosmetic Act . . . . . . . . . . . . . . Option 3.-Establish an Investigatory Monitoring System. . . . . . . . . . . . . Option 4.- Improve Federal Response to New Contamination Incidents . .

II. Environmental Contamination of Food." ",".. . . . . . . ..+00 "00"0 How Food Becomes Contaminated . ......." $", \|^{\prime \prime} \ldots \ldots \ldots \ldots \ldots \ldots \ldots$ Magnitude of the problem $\ldots \ldots \ldots \ldots \ldots \ldots \ldots \ldots \ldots \ldots$

Evidence of Human Illness Resulting From Consumption of

Contaminated Food. . . . . . . . . . . . . . . . . . " $\mathrm{o}^{\prime \prime} ", "$ "..

Polychlorinated Biphenyls. . . . . . . . . . . . . . . . . . . . . .

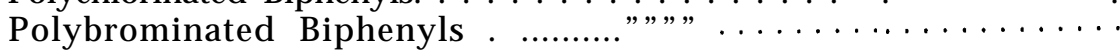
Mercury and Methylmercury . . . . . . . . . . . . . . . . . . . .

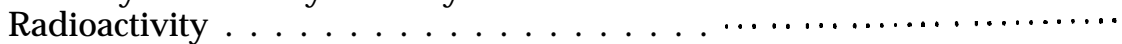

Number of Food Contamination Incidents. . . . . . . . . . . . . . . Economic Impact . . . . . . . . . .."'","'," $\ldots \ldots \ldots \ldots \ldots \ldots \ldots \ldots$

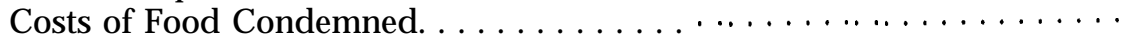

Health Costs... . . . . . . . . . . . . . . . . . . . . .

Distributional Effects and Costs . . . . . . . . . ......... . .

Potential Food Contamination problems. $\ldots \ldots \ldots \ldots \ldots \ldots \ldots$

Conclusions and Issues . . . . . . . . . . . . . . . . . . . . . . . . .

Chapter II References . . . . . . . . . . . . . . . . . . . . .

111. Federal Laws, Regulations, and Programs" ................... 35

Federal Laws. . . . . . . . . . . . ................ 35

Action Levels and Tolerances. . . . . .................. 37

Criteria forgetting Action Levels and Tolerances . . . . . . . . . . . . . 38

Determining Action Levels and Tolerances . . . . . . . . . . . ..... 39

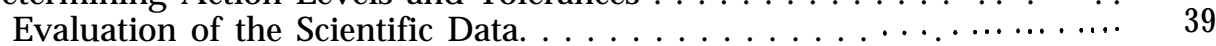

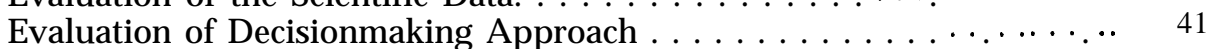

Federal Monitoring programs. . ..................... 42

Food and Drug Administration . . . . . . . . . . . . ........ ...... 42

U.S. Department of Agriculture . . . . . . . . . . . . . .......... 43

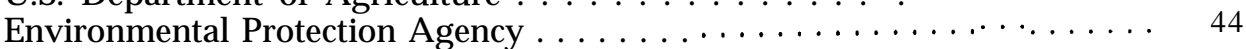

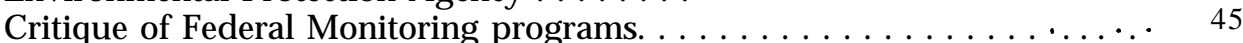

Chapter III References ............................. 46

IV. State Laws, Regulations, and Programs . . . . . . . . . . . . . . . . 49

State Laws . . . . . . . . . . . . . . . . . . . . . . . . . . . . 49

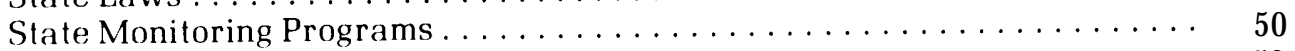

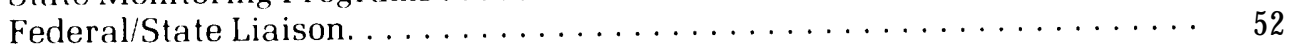

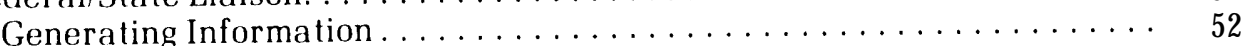

Chapter IV References. . . . . . . . . . . . . . . . . . . 55

V. Methods for Assessing Health Risks...."“","OCO”,OOOO ...... 59

Possible Toxic Effects . . . . . . . . . . . . . . . . . . . . . . . 59

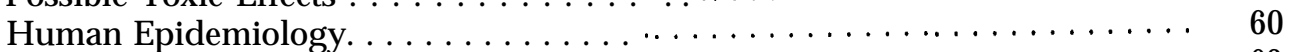

Animal Tests and Other Methods for Toxicological Testing . . . . . . . . . . 62

The Carcinogen Bioassay. . . . . . . . . . . . . . . . . . . . . . . . 62

Short-Term Tests for Mutagenesis and Potential Carcinogenesis. . . . . . . 63 
Interactions of Two or More Substances . . . . . . . . . . . . . . . 65

Extrapolating From High Doses to Low Doses. . . . . . . . . . . . . . . . . . . . . . 67

Chapter Preferences . . . . . . . . . . . . . . . . . . . . . . 69

VI. Methods of Estimating and Applying Costs to Regulatory Decisionmaking . . 73

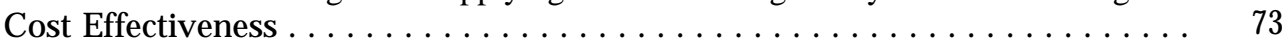

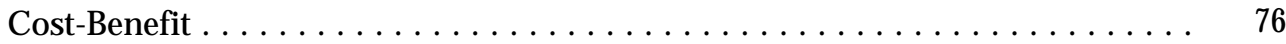

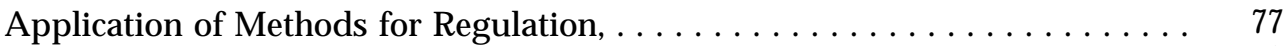

Chapter VI References. . . . . . . . . . . . . . . . . . . . . . 78

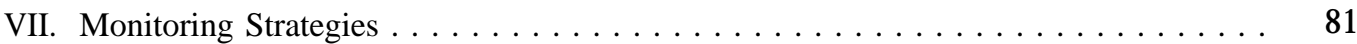

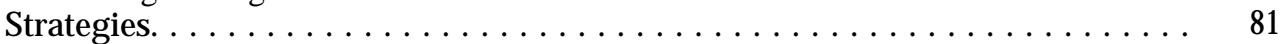

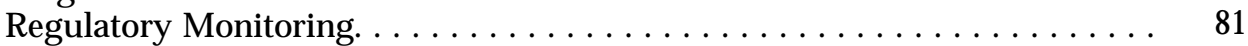

Investigatory Monitoring . . . . . . . . . . . . . . . . . . . . . . . . . 81

Monitoring for Suspected Environmental Contaminants . . . . . . . . . . 82

Monitoring for Uncharacterized Environmental Contaminants . . . . . . . 84

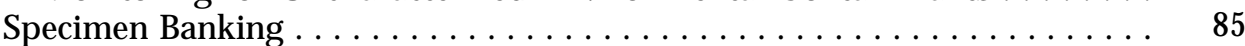

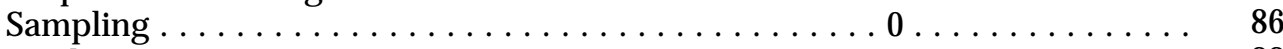

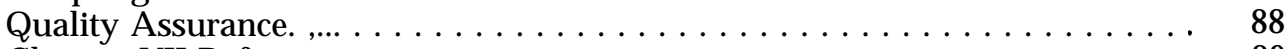

Chapter VII References. . . . . . . . . . . . . . . . . . . . . . . . 89

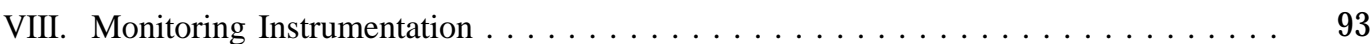

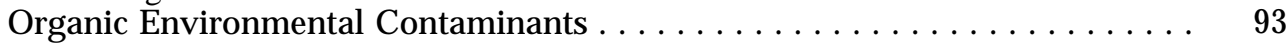

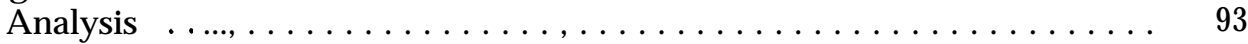

Application to Investigatory Monitoring . . . . . . . . . . . . . . . . . . 96

Detecting and Quantifying Trace Metal Contaminants . . . . . . . . . . . . . . . 97

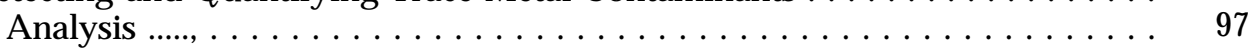

Application to Investigatory Monitoring . . . . . . . . . . . . . . . . 98

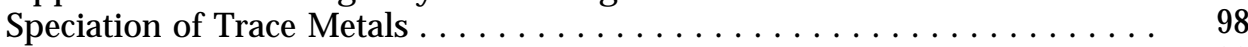

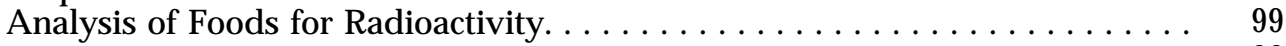

Analysis . . . . . . . . . . . . . . . . . . . . . . . . . . 99

Applications to Investigatory Monitoring. . . . . . . . . . . . . . . . . . . . 102

Estimated Cost to Equip a Laboratory to Conduct Investigatory Monitoring. . 103

Chapter VIII References . . . . . . . . . . . . . . . . . . . . . . 105

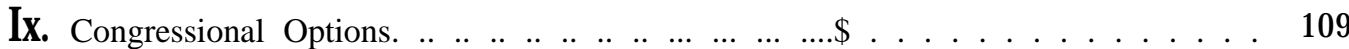

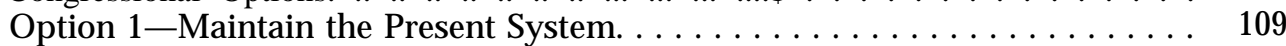

Option 2-Amend the Food, Drug, and Cosmetic Act. . . . . . . . . . . . . . . . 110

Option 2A--Simplify Administrative Procedures. . . . . . . . . . . . . . . . . . 110

Option 2B-Requiret reestablishment of Tolerances . . . . . . . . . . . . . . . . . . 110

Option 2C-Clarify the Role of Economic Criteria . . . . . . . . . . . . . . . . . . . . . . . . . . . . . 111

Option 2D-Establish Regional Tolerances . . . . . . . . . . . . . . . . . . . . 111

Option 3-Establish an Investigatory Monitoring System . . . . . . . . . . . . . 112

Option 3A-Establisha National Monitoring System for Suspected

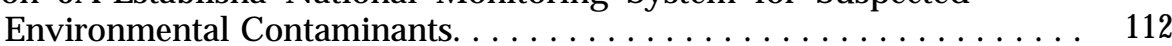

Option 3B-Establisha National Monitoring System for Uncharacterized

Environmental Contaminants. . . . . . . . . . . . . . . . . . . 113

Option 4-Improve Federal Response to New Contamination Incidents . . . . 113

Option 4A-Designate a Lead Agency . . . . . . . . . . . . . . . . . . . . 113

Option 4B-Establish a Center to Collect and Analyze Toxic Substances

Data . . . . . . . . . . . . . . . . . . . . . . . . . . . . . . 114

Appendixes

A. Substances Whose Production or Environmental Release Are Likely to Increase in the Next Ten Years $\ldots \ldots, \ldots, \ldots \ldots \ldots \ldots, \ldots, \ldots, \ldots, \ldots \ldots, \ldots, \ldots, \ldots \ldots$

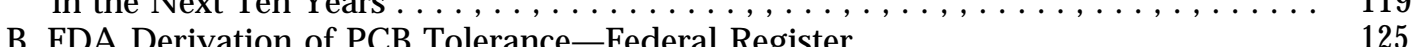


Appendix

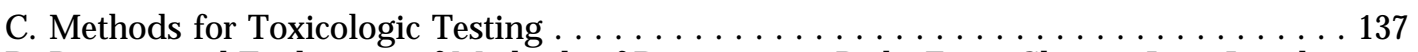

D. Review and Evaluation of Methods of Determining Risks From Chronic Low-Level

Carcinogenic Insult . . . . . . . . . . . . . . . . . . . . . . . . . . . . . . . . 154

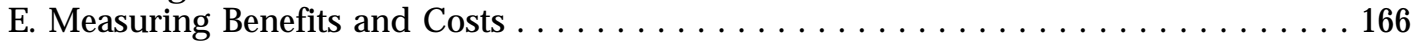

F. Priority Setting of Toxic Substances for Guiding Monitoring Programs . . . . . . . . 177

G. Approaches to Monitoring Organic Environmental Contaminants in Food . . . . . . 187

H. Analytical Systems for the Determination of Metals in Food and Water Supplies . 195

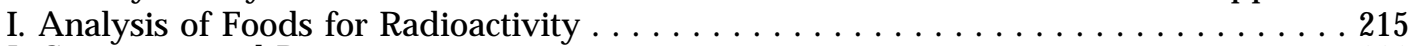

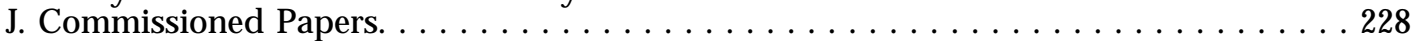

K. Executive Agency Observers at Advisory Panel Meetings. . . . . . . . . . . . . . . 229

\section{LIST OF TABLES}

Table No.

1. Reported Incidents of Food Contamination, 1968-78, by State and Class of

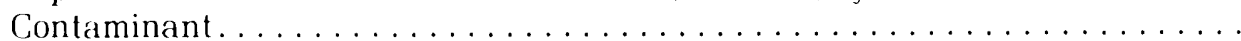

2. Reported Incidents of Food Contamination, 1968-78, by State and Food .......

3. Number of Incidents of Environmental Contaminants of Food Reported by

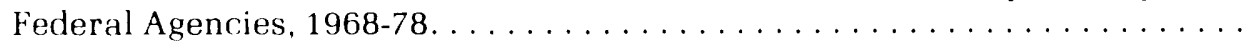

4. Radionuclide Contaminants of Significance for Foods . . . . . . . . . . . .

5. Methylmercury Concentrations in Normally Exposed Populations . . . . . . . . . .

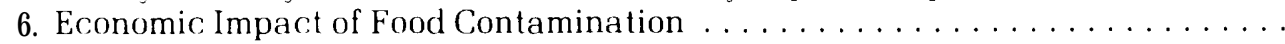

7, Federal Laws and Agencies Affecting Toxic Substances Control . . . . . . . . . .

8. Program Areas and Expenditures for States . . . . . . . . . . . . . .

9. Number of Employees by Category and Education Level in Various State Food and Drug Programs . . . . . . . . . . . . . . . . . . . . .

10. Salary Ranges of Various Chemical Personnel in State Food and Drug Programs

11. Physical Facilities and Key Equipment in 114 State Food and Drug Laboratories

12. Total Number of Samples, Sample Determinations, and Man-Hours by Food Commodity Categories With Number and Percentage of States Reporting

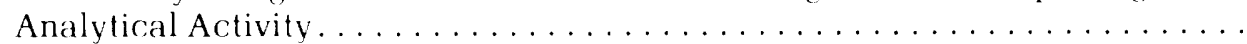

13. Combined Effects of Cadmium. Mercury, and Lead . . . . . . . . . . . . .

14. Virtually Safe Doses Computed From Three Different Data Sets and Three Different Models . . . . . . . . . . . . . . . . . . . . . .

15. Extra Lifetime Risks of Cancer Associated With Consumption of PCBs in Food .

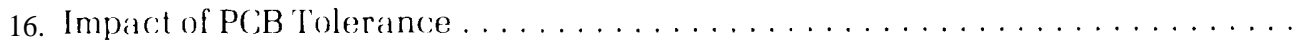

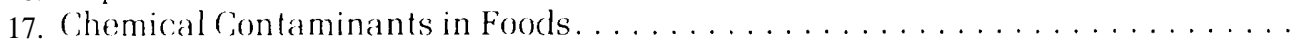

18. Techniques Available for Qualitative and Quantitative Organic: Analysis .....

19. Techniques Available for Qualitative and Quantitative Trace Metal Analysis .

20. Tvpical Limits of Detection for Radionuclide Measurement . . . . . . . . . . . .

21, Estimated Costs to Equip a Laboratory to Conduct Uncharacterized Monitoring

A-1. Trapped Organic Compounds and Aromatic Units in Coal . . . . . . . . . . . .

D-1. Comparison of Lower 97.5-Percent Confidence I,imit on Virtually Safe Doses From Gamma Multihit Model Given in to Virtually Safe Doses From Best Fitting Gamma Multihit Model With $k=1 \ldots \ldots \ldots \ldots \ldots \ldots \ldots \ldots$

G-1. Techniques Available for Qualitative and Quantitative Organic Analysis ....

G-2. Fstimated Space, Manpower, and Cost Associated With the Analysis of Priority Poilutants in Tissue and Water Samples Acoording to FPA Analytical

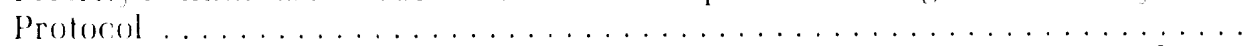

G-3. Estimated Space, Manpower, and Cost Associated With the Monitoring of Unknown Potentially Toxic Compounds in Addition to the EPA Priority

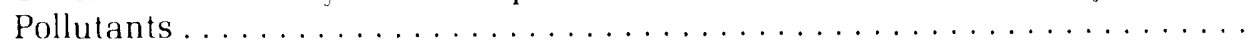

H-1. Determination Limits for Neutron Activation Analyses . . . . . . . . . . . . . .

H-2. Determination Limits for Flame Atomic Absorption and Emission and Furnace Atomic Absorption . . . . . . . . . . . . . . . . . . . . .

Page 
Table No.

H-3, Multielement Atomic Emission Determination Limits for Two Common Plasma

Excitation Systems. . . . . . . . . . . . . . . . . . . . . . . . . . . . . . . 204

H-4. Determination Limits for X-Ray Fluorescence Techniques. . . . . . . . . . . . . . . . 205

H-5. List of Sample Preparation Facilities Required . . . . . . . . . . . . . . . . . . . . . . 209

H-6, Summary of Cost-Productivity Estimates for Various Analytical Techniques . . . 210

I-1. Costs for Space, Furnishings, Equipment, and Supplies .,,............. 225

\section{LIST Of FIGURES}

Figure No. $\quad$ Page

1. Distribution of PCB Contamination $\ldots \ldots \ldots \ldots \ldots \ldots \ldots \ldots$

2. Distribution of States Responding to Questionnaire . . . . . . . . . . . . . . . 24

3. State Food Laws. . . . . . . . . . . . . . . . . . . . . . . . . . . . . 49

4. Scope of Investigation: Distribution of Materials $\ldots \ldots \ldots \ldots \ldots \ldots \ldots$

5. Steps in the Ames Spot Test. . . . . . . . . . . . . . . . . . . . . . 65

6. A General Scheme for the Qualitative and Quantitative Analysis of the Organic EPA Priority Pollutants in Semisolid Foods . . . . . . . . . . . . . . 94

7. Comparison of the Gas Chromatographic Analysis of a Standard Mixture of Polychlorinated Biphenyls on Two Types of Gas Chromatographic Columns . . . 96

D-I, Illustration of Why the Dose-Response Curve Should Be Linear at Low Dose in the Presence of Background Carcinogenesis ... ... . . . . . . . . . . . . . . 156

D-2. Typical Fit of Mantel-Bryan Curve to Experimental Data , . . . . . . . . . . . . . . . 158

D-3. Comparisons of "Safe" Doses Computed From the Mantel-Bryan Procedure and From a Procedure Based on the Multistage Model . . . . . . . . . . . . . . . , 161

D-4a, Example of Data Exhibiting Downward Curvature . . . . . . . . . . . . . . . . . . . . . . . . . . . . . . . . . .

D-4b, Example of Data Exhibiting Upward Curvature. . . . . . . . . . . . . . . . . . . . . . . . . . 162

E-1. Analysis of a Shift in Supply .-. , . . . . . . . . . . . , . . . . . . . , ., , ., . . . . . 0.170

G-I. A Simplified Diagram for the Qualitative and Quantitative Analysis of the Organic EPA Priority Pollutants . . . . . . . . . . . . . . . . . . . 189

G-2. A General Scheme for the Qualitative and Quantitative Analysis of the EPA Organic Pollutants in Semisolid Foods . . . . . . . . . . . . . . . . . . . . ., 189

G-3. General Analytical Scheme To Detect and Monitor New Trace Organics and Priority Pollutants in Food and Water Samples . . . . . . . . . . . . . . . . . . . . . 192

H-1. Flow Diagram of the General Operational Protocol of a Monitoring Laboratory 197

1-1. Comparison of Gamma Spectra Taken With a Sodium Iodide Detector and a Germanium Diode Detector.. 
Chapter I

\section{Summary}




\section{Summary}

The environmental contamination of food is a nationwide problem. A number of recent incidents dramatically illustrate the potential health hazards and economic harm that can be caused by such contamination-animal feeds in Michigan contaminated by polybrominated biphenyls (PBBs), the Hudson River contaminated by polychlorinated biphenyls (PCBs), and Virginia's James River contaminated by kepone.

These are some of the more serious of the 243 food contamination incidents identified in an OTA survey of the 50 States and 10 Federal agencies. These incidents have occurred in every region of the country. They have involved all categories of food. While the OTA survey clearly shows the national character of such contamination, the true extent of the problem is still unknown.

The latest major food contamination incident-one not included in the OTA survey-graphically points up the ominous dimensions of the problem. PCBs from a damaged transformer contaminated animal fats at a packing plant in Billings, Mont. The plant used the adulterated fats to produce meat and bone meal that were sold both to feed manufacturers and directly to farmers. The contaminated feed spread through at least 10 States-polluting poultry, eggs, pork products, and a variety of processed foods (including strawberry cake). The result: contaminated food found in 17 States, and hum dreds of thousands of pounds of food products seized or destroyed.

This assessment, undertaken at the request of the House Committee on Interstate and Foreign Commerce, examines the adequacy of current Federal and State efforts to deal with the environmental contamination of food. In particular, the study evaluates the effectiveness of 1) Federal and State monitoring systems in detecting contamination episodes before they reach crisis proportions, and 2) Federal efforts to regulate contaminations. The study explores alternative approaches to the problem and presents policy options for Congress.

Environmental contaminants in food fall into three categories - synthetic or natural organic chemicals, metals or their organic and inorganic derivatives, and natural or synthetic radioactive substances. Such contaminants are regulated under the Federal Food, Drug, and Cosmetic Act. To regulate them under the law, the Food and Drug Administration (FDA) defines environmental contaminants as "added, poisonous, or deleterious" substances that cannot be avoided by good manufacturing practices, and that may make food injurious to health.

Unlike food additives, environmental contaminants inadvertently find their way into the human food supply (including sports fish and game). They can enter food directly or indirectly as a result of such human activities as agriculture, mining, industrial operations, or energy production. In no instance is their presence in food ever intended. 


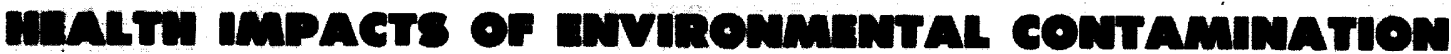

Four factors determine whether and how seriously the environmental contamination of food will affect human health: the toxicity of the contaminant, the amount of the substance in the food, the amount of the contaminated food eaten and the physiological vulnerability of the individual or individuals consuming the food.

Based on other countries' experiences, there is considerable evidence of human illness caused by the consumption of food containing various organic chemicals and metals. In such cases, the level of the contaminant in food exceeded the levels usually found in the U.S. food supply. The effects of mercury poisoning are well-documented. The best known case involved the consumption of mercury-contaminated fish from Japan's Minamata Bay. Some of the offspring of exposed mothers were born with birth defects, and many victims suffered central nervous system damage.

Another incident in Japan stemmed from the inadvertent contamination of rice oil by PCBs. The consumption of food cooked with this oil resulted in 1,291 cases of so-called "Yusho disease" - a condition marked by chloracne (a severe form of acne), eye discharges, skin discoloration, headaches, fa- tigue, abdominal pains, and liver and menstrual disturbances,

No such mass-poisoning episodes have occurred in the United States. But there are studies indicating that present levels of some environmental contaminants may cause physiological changes. For example, the accidental contamination of animal feed in 1973 exposed most of the population of Michigan to PBB in dairy products and other foods. Evidence on what impact this exposure has had on human health is conflicting, although some disparities in white blood cell function have been noted in farm families, The long-term significance of these physiological changes is not yet known.

The clinically obvious harmful health effects of radiation are usually associated with massive, high-level exposures. Past cases of radioactive contaminated foods have involved relatively small amounts of radioactive substances with low dose rates. Generally, the young are most sensitive to radiation exposure. However, since any amount of radiation is potentially harmful, prudent public policy must assume that any unnecessary exposure to high-energy radiation should be avoided.

\section{ECONOMLC IMPACTS OF contAMINATION}

The economic impacts of a contamination incident have traditionally been stated in terms of the estimated dollar value of the resulting food loss. Only limited data on such costs are available. Dollar value estimates for condemned food were available for less than 30 percent of the contamination episodes noted in the OTA survey. Thus, the real cost of environmental contamination of food during the 1968-78 decade is at least several times the $\$ 282$ million reported to OTA.

The loss of food only partially reflects the total economic impact of environmental contamination, Health and "distributional" costs are also involved. The health costs include medical expenses and lost workdays from illness resulting from food contamination incidents. Since the health effects may not immediately be evident, the expected illnesses or deaths from an episode are usually estimated on the basis of available toxicity data for a particular contaminant. In other words, estimated health costs are more likely to be projections than actual figures based on known cases of illness or death.

The "distributional" costs of environmental contamination disclose the expenses or losses incurred by affected businesses, individuals, and government bodies. These might include farmers, fishermen, food processors, 
animal feed suppliers, chemical companies, consumers, and local, State, and Federal agencies. Although the individual organizations suffering such losses are usually identi- fied, their actual dollar losses are not known. To understand who is bearing the major economic brunt of a contamination episode, actual cost data are required.

\section{MAJ OR PROBLEMS IN IDENTIFYING ENVIRONMENTAL CONTAMINANTS}

To determine whether an environmental contamination incident has occurred, it is necessary to establish the presence of the contaminant in food. In some instances, people or animals have become ill before the responsible contaminant was identified. No one knew or even suspected that the particular substance was present in food. This has been the pattern in many major contamination incidents - those involving PBBs, PCBs, and mercury.

Our regulatory monitoring system has failed to detect such environmental contaminants as they entered the food supply. Thus, this assessment identifies and evaluates other approaches for monitoring either food or the environment for toxic substances that may harm human health. The ultimate objective of monitoring is to prevent or minimize human exposure to environmental contaminants in food.

The only sure way to prevent this kind of contamination is to make certain that toxic substances are not released into the environment. There are various Federal environmental laws that are designed to limit such releases. But the laws and regulations are not likely to prevent the deliberate or accidental misuse or disposal of the thousands of toxic substances manufactured in the United States.

The problem is compounded by disposal and handling practices that was accepted in the past but are now recognized as posing serious environmental hazards-hazards that will persist for many years to come. The toxic chemical waste dump at the Love Canal near Niagara Falls, N. Y., clearly illustrates the threat. According to Environmental Protection Agency (EPA) estimates, there are 1,200 to 2,000 of these abandoned chemical and radioactive waste sites in the United States that pose an imminent danger to human health and will cost as much as $\$ 50$ billion to clean up. As long as these substances remain in the environment, the potential for food contamination exists.

\section{PROBLEMS OF REgulating ENVIRONMIENTAL ContaminaNTS}

Once an environmental contaminant is found in food, limits are established to control and restrict its presence. Such regulations are set up and enforced by FDA for food traded in interstate commerce, and by State agencies for food produced and sold within a State. In either case, the aim is to limit the public's exposure to a particular contaminant.

Key factors involved in such regulation are time and information. After a contaminant is identified. authorities must have information on its toxicity, the amount present in the food, and how much and what kinds of food are contaminated. By monitoring the food supply for the contaminant, regulators can determine the level and extent of the contamination. With this information and necessary toxicity data, they can establish regulatory limits for the contaminant in food.

However, this kind of information generally takes time to generate-usually longer than the public is willing to wait in the event of a food contamination incident. As a result, 
authorities are often pressed to set regulatory limits before they have enough time to develop information on the nature and extent of the contamination.

\section{FINDINGS AND conchusions}

This assessment has focused on two central problems: regulating environmental contaminants and identifying environmental contaminants. Following are major findings and conclusions growing out of this assessment.

- FDA relies on action levels rather than tolerances to regulate environmental contaminants in food.

Under the Food, Drug, and Cosmetic Act, FDA is given authority to set tolerances for the amount of an unavoidable contaminant permissible in food. However, the procedures required to set a tolerance are complex, cumbersome, and time-consuming. Therefore, FDA relies on action levels, informal judgments about the level of a food contaminant to which consumers may safely be exposed.

Action levels are administrative guidelines that can be developed and promulgated more easily and quickly than tolerances. Action levels are used when scientific data are incomplete. Public input is not required. They are used when new information is likely to be forthcoming that might alter the level. FDA is under no constraints to review action levels or to replace action levels with formal tolerances. FDA has sometimes lowered or raised action levels as new data became available. FDA is now in the process of lowering the PCB tolerance.

- No policy exists defining the relative weights to be given to the evidence when setting an action level or tolerance.

In setting an action level or tolerance, FDA takes into account short- and long-term toxicological data, available information on the levels of the contaminant in food, the amount of contaminated food consumed by various population groups, the level that can be measured, and the potential impact of various action levels or tolerances on the national food supply. Generally, the more information about a particular factor, the greater its in- fluence. Because the amount and quality of information available when FDA encounters an environmental food contamination problem are inevitably unpredictable, it does not predetermine the weighting of various factors. However, FDA maintains that the public health factor outweighs all others in its considerations.

The Food, Drug, and Cosmetic Act does not specify the role that the costs of a regulatory decision should play in setting a tolerance or action level. The Act does require that FDA take into account the extent to which a substance cannot be avoided in food production. FDA interprets this requirement as justification for weighing the costs of food condemned against the health benefits derived from a tolerance.

\section{- To assess human risk from exposure to chemicals, FDA and EPA rely on already- existing animal studies and epidemiologi- cal evidence derived from previous hu- man exposures,}

When a new environmental contaminant is discovered in food, regulatory agencies are under intense pressure to act to protect the public. FDA and EPA (if the contaminant is a pesticide) review the available literature on the contaminant and calculate an action level based on that evidence. Rarely are new studies commissioned-even when the data are inadequate.

New human epidemiological studies and conventional 2-year animal studies are of little immediate help because so much time is required to generate results. However, toxicologists have developed a variety of tests that can evaluate a substance's possible toxic effects in 90 days or less. Some of these shortterm tests measure the potential of a substance to produce mutations and possible cancer, 
Short-term tests could be used more widely in screening environmental contaminants to determine whether they are mutagens or potential carcinogens. Although the results of such tests do not provide the data needed to set an action level or tolerance, they still can alert regulators to latent dangers that require further investigation.

Conventional 2-year animal studies (which usually entail an additional year for data analysis) would continue to serve an important role in the setting of tolerances. If data from a carcinogen bioassay are available at the time an environmental contaminant is discovered in food, the information can prove crucial in reaching a regulatory decision. If data were nonexistent or inadequate, a newly commissioned carcinogen bioassay could be used to revise an initial action level.

Epidemiological studies would remain useful for confirming suspected chronic effects of a toxic substance to which a population has unknowingly been exposed over a period of time. They can also confirm retrospectively or refute the adequacy of regulatory actions.

No currently available toxicological testing methods or statistical interpretation techniques are adequate for evaluating the combined effects of low-level exposure to toxic substances. Indeed, there are no satisfactory techniques for testing the interactions of more than two substances.

- Current monitoring at both Federal and State levels is regulatory, designed to ensure that substances in food do not exceed prescribed limits. Little effort is made to detect and identify substances in the food supply for which no action levels or tolerances exist.

Technology now exists that would make possible a national investigatory monitoring system to detect unregulated chemicals as they enter the food chain. Such advanced technology is available in some Federal regulatory monitoring laboratories and in a limited number of State labs. It is not routinely employed in Federal or State regulatory monitoring.

The goal of food monitoring is to protect consumers by determining short- and longterm trends in the levels of various chemicals in food and the environment. Investigatory monitoring could be designed to complement already-existing regulatory monitoring. Each of these approaches could be complemented by specimen banking-the regular collection and storage of samples that could be later analyzed if a new contaminant is found in food. EPA and the National Bureau of Standards are now working towards developing such a specimen-banking program.

However, food sampling may not be the best approach to investigatory monitoring. To discover a substance as it enters the environment and before it gets into the human food supply, it is necessary to monitor water, soil, air, river sediments, and nonfood organisms.

- Management of food contamination incidents is hindered by the complexity of the food system, the rapidity with which food is moved through the system, and failures by State and Federal agencies to coordinate their information-gathering activities.

Many food contamination incidents initially fall under State jurisdiction. Technically, the Federal Government does not become involved unless requested by a State or until contaminated food enters interstate commerce. This country's food marketing system is complex. Most food produced or processed within a particular State is distributed for consumption in other States. Thus, most environmental contamination incidents are likely to become interstate problems. Figure 1 illustrates the extent of food contamination that can occur from a single source of contamination, in this case PCB-contaminated animal feed from a meatpacking plant in Billings, Mont.

The number of State and Federal agencies involved complicates the generation and dissemination of scientific information on the 


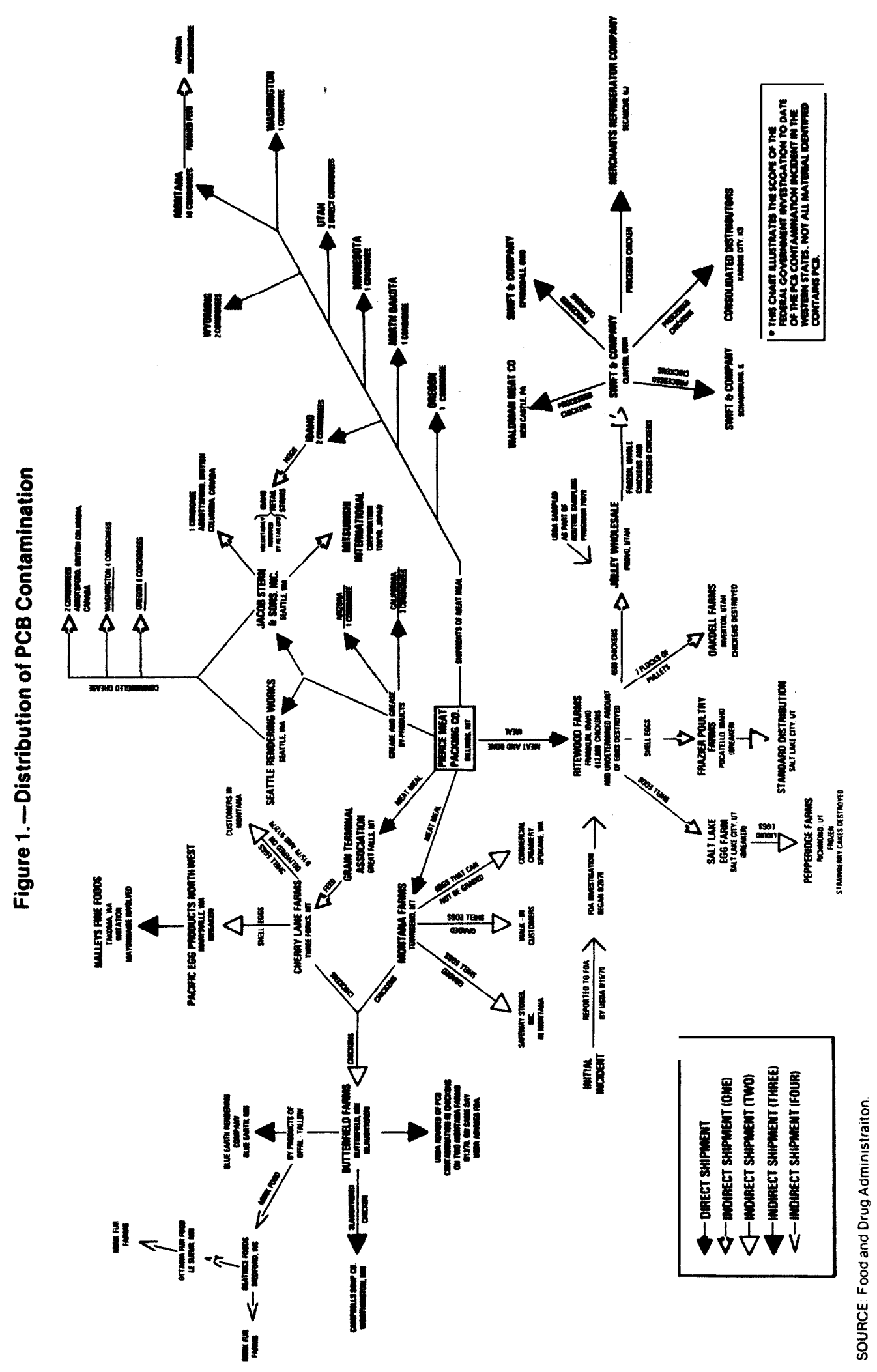


toxicological and chemical properties of the contaminant, the amount and type of food contaminated, and the concentration of the substance in food. At least three Federal agencies (EPA, FDA, and the U.S. Department of Agriculture (USDA)), each with different responsibilities, may provide technical assistance. At the State level, departments of health, agriculture, and the environment may share accountability for regulating environmental contaminants in food.

In the absence of a clear authority to coordinate activities of various agencies, the po- tential exists for breakdowns in communication. This was the case in the recent PCB contamination of animal feeds in 10 Western States. The Idaho Department of Agriculture did not inform the Idaho Department of Health and Welfare of the PCB contamination. USDA would report the results of its investigations only to the Idaho Department of Agriculture. EPA attempted to determine the source of the PCBs by analyzing air and water samples, but failed to report its negative results to the State.

\section{CONGRESSIONAL OPTIONS}

There are four basic options for Congress to consider regarding the Federal response to the environmental contamination of food. Each is discussed in greater detail in chapter IX. Congress can:

1. Allow the present system to continue by taking no action. The present system consists of regulatory monitoring and the establishment of action levels (and occasionally tolerances) for environmental contaminants in food.

2. Amend the Food, Drug, and Cosmetic Act to specifically address the unique problems posed by environmental contamination of food.

3. Establish a national investigatory monitoring system.

4. Improve the Federal response to new contamination problems by designating a lead agency or establishing a center to orchestrate the delivery of Federal assistance to affected States.

\section{Option 1 \\ Maintain the present System}

Pros: There are two principal advantages in maintaining this system. No additional appropriations or legislation are required. No changes in existing regulations are necessary.
Cons: The time needed to identify an environmental contaminant in food and take corrective action would not be shortened if the current system were retained. Moreover, action levels and tolerances permit a certain level of contaminant to be present in food. If tolerances or action levels are not reduced, little effort will be made to eliminate the contaminant. There is no requirement for review of an action level once it is established. Thus, FDA is under no pressure to actively seek out new data to verify the appropriateness of an existing action level. Finally, States have no clearly defined authority to turn to when they suspect environmental contamination of food.

\section{Option 2 \\ Amend the Food, Drug, and Cosmetic Act}

An amendment to the Food, Drug, and Cosmetic Act could contain one or more of the following changes. Each change is discussed in greater detail in chapter IX.

- Congress could amend the Food, Drug, and Cosmetic Act to simplify the administrative procedure for setting tolerances. The change could be modeled after section 553 of the Administrative Procedures Act. This would encourage FDA to move from action levels to toler- 
ances, thus bringing more public participation into the process,

- Congress could amend the Food, Drug, and Cosmetic Act to require the establishment of a tolerance within a specified time after the setting of an action level. This would encourage the FDA to gather additional information on a contaminant's toxicity and the public's exposure. It would result in a definitive tolerance that FDA could enforce with less concern over legal challenge.

- Congress could clarify to what extent economic criteria can be used in setting tolerances for environmental contaminants in food,

- FDA could be granted authority to set regional tolerances. This would provide FDA with flexibility to set different levels for different regions based on expected levels of exposure, regional levels of contamination, and local eating patterns.

Pros: Since its passage in 1938, the Food, Drug, and Cosmetic Act has been amended several times to deal with new problems of food regulation. Congress has never directly addressed the environmental contamination of food. There are several unique characteristics of this problem that could be clarified through an amendment dealing specifically with the environmental contamination of food.

Cons: Even though the Food, Drug, and Cosmetic Act does not contain provisions on environmental contaminants, FDA has been able to regulate them through interpretation of sections 402 and 406.

\section{Option 3 \\ Establish an Investigatory Monitoring System}

Congress could establish a national investigatory monitoring system based on monitoring for either suspected or uncharacterized environmental contaminants. Some chemicals are not regulated by action levels or tolerances but are suspected to be dangerous to humans if consumed in food. Uncharacterized environmental contaminants are substances that may have entered the food sup ply, but are not regulated or suspected food contaminants. A system that combines elements of both approaches could also be set up. Because any of these monitoring approaches would require some research and development before going into full operation, Congress could choose to establish a pilot program. Such a program would spur research and development and assess the feasibility and cost effectiveness of the various approaches.

Pros: Investigatory monitoring would increase the probability of detecting unregulated substances in food. Present food-monitoring efforts are not designed to detect unregulated environmental contaminants in food. The limited amount of investigatory monitoring that does exist is primarily concerned with trace metals. To identify new contaminants as they enter the food supply, more of this type of monitoring is needed.

Cons: The costs of setting up an investigatory monitoring program could be large, and there is no certainty that the sampling plan would identify all environmental contaminants before they enter the food chain. Furthermore, investigatory monitoring relies on sophisticated instrumentation that is generally not found in Federal or State monitoring laboratories,

\section{Option 4 \\ improve Federai Response to New Contamination Incidents}

The Federal response to new contamination problems has been hampered by the multiplicity of agencies with regulatory or monitoring responsibilities for the environment and for food. Congress could designate a lead agency or establish a center to orchestrate delivery of Federal assistance to affected States.

Pros: With a clearly delineated agency or center, States suspecting contamination of 
food would have one reliable Federal source for generating, evaluating, and disseminating technical information. Response time might be shortened, duplication of effort reduced, and effective management of the incident enhanced.

Cons: Better coordination among FDA, USDA, and EPA could accomplish the same goals without the expense of establishing a new research center. Historically, the major impediment to timely Federal response to chemical contamination of food was lack of awareness that food contamination had taken place. When contamination became apparent and one or more Federal agencies were alerted, response was rapid. Furthermore, establishment of a lead agency or a new cen- ter would not ensure that information would be generated more quickly than is now the case.

Options 2 through 4 are not mutually exclusive. If Congress wishes to put greater emphasis on protecting consumers from contaminated food, one or more could be chosen. For example, Congress could decide to simplify the administrative procedures for setting tolerances (Option 2), require the setting of a tolerance at some specified time after an action level is set (Option 2), establish a pilot program of investigatory monitoring for organic chemicals (Option 3), and designate FDA the lead agency to deal with new contamination problems (Option 4). 
Chapter II

Environmental Contamination of Food 


\section{Environmental Contamination of Food}

Maintaining an adequate, safe food supply has been a major goal of the Federal Government since 1906, when the first Federal food and drug law was signed into law. Historically, chemicals such as salt, sugar, and wood smoke have been used to preserve foods. Modern food technology relies extensively on the use of chemicals not only for preservation but also to produce appealing colors, flavors, aromas, and textures.

Most developed countries now have food laws designed to permit the use of such chemicals in food under conditions judged to be safe. These chemicals are not considered adulterants or contaminants and are classed as intentional additives. Other chemicals may enter food as a result of their use in food production, handling, or processing. Such substances maybe legally permitted if they are unavoidable under good manufacturing practices and if the amounts involved are considered safe. These chemicals are classed as incidental additives. The presence of both these classes of chemicals in food is controlled by regulation.

Environmental contaminants include substances from natural sources or from industry and agriculture. Many of the naturally occurring contaminants in food are of microbiological origin and consist of harmful bacteria, bacterial toxins, and fungal toxins. (Aflatoxin, a contaminant of peanuts and grains, is an example of a fungal toxin or mycotoxin. ) The second category of environmental contaminants includes organic chemicals, metals and their complexes, and radionuclides. Only those environmental contaminants introduced into food as a result of human activities such as agriculture, mining, and industry are considered in this assessment.

The environmental contamination of food is a result of our modern, high-technology society. We produce and consume large volumes of a wide variety of substances, some of which are toxic. It is estimated that 70,000 chemicals may currently be in commercial production in the United States and that 50 of these chemicals are manufactured in quantities greater than 1.3 billion lbs per year. Seven percent of this country's gross national product (GNP), $\$ 113$ billion per year, is generated by the manufacture and distribution of chemicals (1). During the production, use, and disposal of these substances, there are opportunities for losses into the environment. For example, the Environmental Protection Agency (EPA) estimates that there are more than 30,000 chemical and radioactive waste disposal sites. Of these, 1,200 to 2,000 are considered threats to human health (2).

Environmental contamination of food takes two forms: long-term, low-level contamination resulting from gradual diffusion of persistent chemicals through the environment, and relatively shorter term, higher level contamination stemming from industrial accidents and waste disposal.

An example of low-level contamination is polychlorinated biphenyls (PCBs). This group of substances was widely used in transformers and capacitors, as heat-transfer fluids, and as an additive in dyes, carbon paper, pesticides, and plastics (3). Although production was halted in 1977, PCBs remain an ubiquitous, low-level contaminant of many foods, especially freshwater fish.

An example of the second type of contamination is polybrominated biphenyls (PBBs) in 
dairy products and meat. PBBs, a fire retardant, were accidentally mixed into animal feed. Dairy cattle that were fed the contaminated feed produced contaminated milk. The distinctions between the two types of food contamination are not exclusive. For exampie, PBBs have now become a long-term, low- level contaminant in Michigan because they are very stable and resistant to decay. Animals raised on farms affected by the original feed contamination are now contaminated by the PBB residues remaining in the pastures and farm buildings.

\section{HOW FOOD BECOMES CONTAMINATED}

Chemicals contaminate foods through different routes depending on the chemical and its physical properties, its use, and the source or mechanism of contamination.

Organic substances that have contaminated food have been either industrial or agricultural chemicals. Pesticides are the only agricultural chemicals known to be environmental contaminants in food (see tables 1-3). A pesticide becomes an environmental contaminant when it is present in foods for which the application or use of the substance has not been approved. Livestock, poultry, and fish can be contaminated when application or manufacturing of pesticides occurs in the vicinity or when residues are transported through the environment. Improperly fumi- gated railroad cars, trucks, ships, or storage lbuildings used for transport or storage of lhuman food and animal feed are also sources of environmental contamination. The interiors are sprayed or fumigated with pesticides, iand if not sufficiently aired, contamination of the food or feed occurs.

The manufacture of organic chemicals proiduces sludges, gases, and liquid effluents of varying chemical complexities. The usual waste disposal methods (sewage systems, incineration, landfill) are unable to prevent organic residues from entering the environment in spite of Federal laws and corresponding regulations governing disposal. The routes include the atmosphere, soil, and surface or ground water.

Table 1.- Reported Incidents of Food Contamination, 1968-78, by State and Class of Contaminant

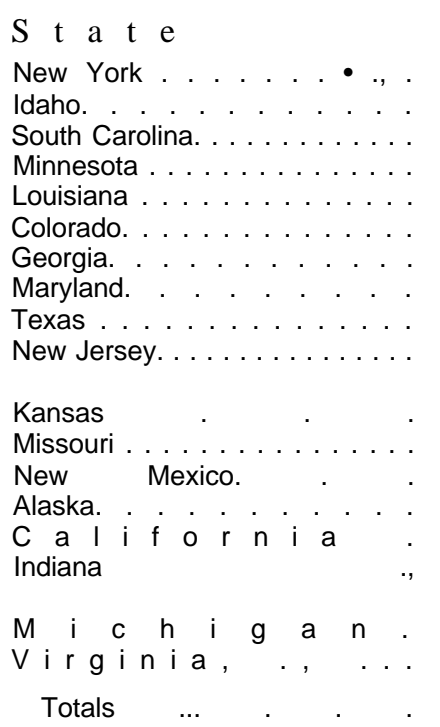

"Several conservatively estimated as one SOURCE Of fice of Technology Assessment

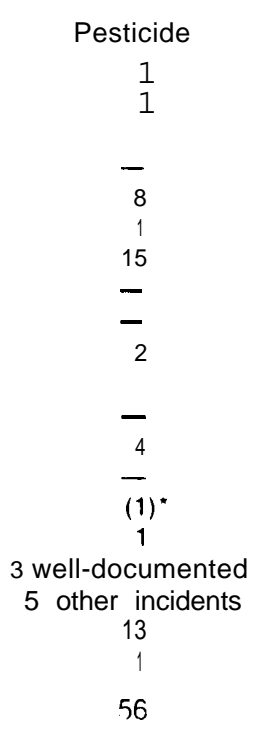

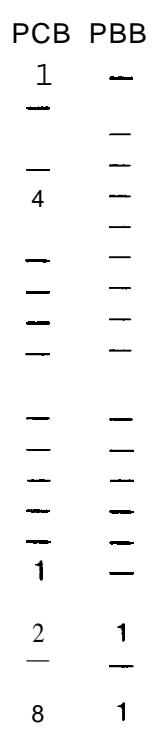

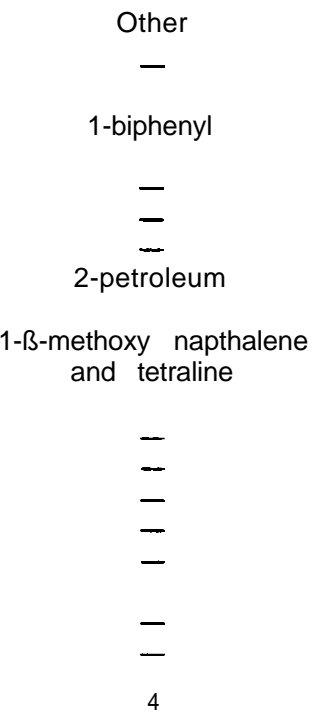


Table 2.- Reported Incidents of Food Contamination, 1968-78, by State and Food

\begin{tabular}{|c|c|c|c|c|c|c|c|}
\hline State & Dairy & Eggs & $\begin{array}{c}\text { Fruit/ } \\
\text { Vegetable }\end{array}$ & Fish/shellfish & Grain & $\begin{array}{c}\text { Game/meat/ } \\
\text { poultry }\end{array}$ & Incidents \\
\hline 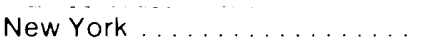 & - & - & - & 3 & - & - & 3 \\
\hline Idaho.................. & 1 & - & - & 1 & - & 1 & 3 \\
\hline South Carolina. . . . . . . . . . . . & - & - & - & - & 1 & & 1 \\
\hline Minnesota . . . . . . . . . . . & - & - & - & 1 & - & & 1 \\
\hline Lovisiana . . . . . . . . . . . . . . & 5 & 1 & 1 & 9 & 1 & & 17 \\
\hline Colorado................. & $1^{a}$ & - & $1^{a}$ & - & - & & $2^{a}$ \\
\hline Georgia. . . . . . . . . . . . & - & - & 15 & - & - & & 15 \\
\hline Maryland ................. & - & - & - & 3 & - & & 3 \\
\hline 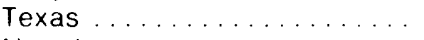 & - & - & - & 1 & - & - & 1 \\
\hline New Jersey. . . . . . . . . . . . . . . . & - & - & 2 & 2 & - & - & 4 \\
\hline Kansas . . . . . . . . . . . . & - & - & - & - & 1 & & 1 \\
\hline Missouri . . . . . . . . . . . . & 4 & - & - & - & & - & 4 \\
\hline New Mexico . . . . . . . . . . . . . . & - & - & - & - & - & 1 & 1 \\
\hline California . . . . . . . . . . & - & - & 1 & 2 & - & 1 & 4 \\
\hline 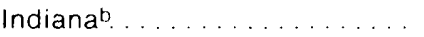 & 1 & 2 & - & - & - & 1 & 9 \\
\hline Michigan. . . . . . . . . . . . . & 7 & - & 2 & 4 & - & 4 & 17 \\
\hline Alaskac . . . . . . . . . . . & - & - & - & - & - & & 1 \\
\hline Virginia . . . . . . . . . . . & - & - & - & 1 & - & & 1 \\
\hline Totals . . . . . . . . . . . . & 19 & 3 & 22 & 27 & 3 & 8 & 88 \\
\hline
\end{tabular}

Table 3.- Number of Incidents of Environmental Contaminants of Food Reported by Federal Agencies, 1968-78

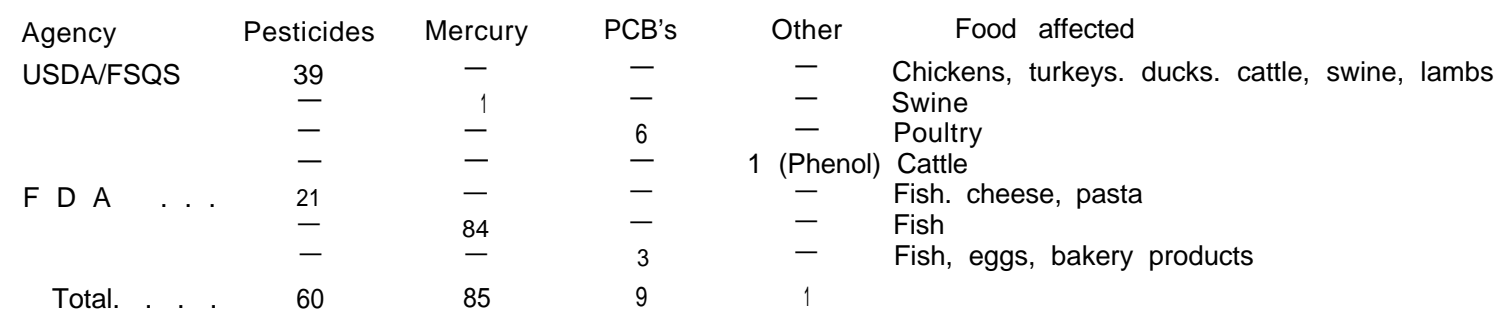

SOURCE Off Ice of Technology Assessment

Metals can be released into the environment in several ways. The mining and refining processes produce dust and gases which enter the atmosphere. Metallic salts formed during recovery and refining processes can escape as waste products into surface and ground water. Sewage sludge used as fertilizer on agricultural land also poses a potential food contamination problem. Trace metals present in the sludge can be taken up by crops grown on treated soil. Cadmium is the trace metal in sludge that currently generates the greatest concern.

Radioactivity in food stems from three sources: natural radioactivity. releases from operation of nuclear reactors and processing plants, and fallout from nuclear weapons tests. The primary route by which food becomes contaminated is the deposition of airborne material on vegetation or soil. The subsequent fate of the radionuclide is determined by its chemical and physical nature and whether it is absorbed and metabolized by plants or animals. Natural radioactivity may become a concern when ores containing radioactive substances are mined and processed. The products or wastes may concentrate the radionuclides. Examples of this are uranium tailings, phosphate rock waste, or slags from phosphorus production. Radium may enter the food chain when it dissolves in 
ground water and is taken up through plant roots.

Nuclear reactors normally release radioactive noble gases that do not contaminate foods. Reactors do contain large inventories of fission products, transuranics, and other activation products. Accidental releases can contaminate vegetation by deposition of particles on leaves and soil, or through water. Gaseous releases would most likely involve the volatile elements such as iodine and tritium, or those with volatile precursors, such as strontium-90 and cesium-137. Aqueous releases would follow failure of the onsite ion exchange cleanup system. Any of the watersoluble elements could be involved. Table 4 summarizes the radionuclide contaminants of significance for foods.

Nuclear waste-processing plants could also have either gaseous or aqueous releases. In this case, the fission products are aged before processing, and iodine and the gaseous precursor radionuclides are not released. Tritium and carbon-14 are the major airborne products, while the waterborne radionuclides are the same as for reactors.

Atmospheric nuclear weapons tests distribute their fission products globally. Local deposition depends on the size of the weapon and the conditions of firing (high altitude, surface, or underground).

Table 4.- Radionuclide Contaminants of Significance for Foods

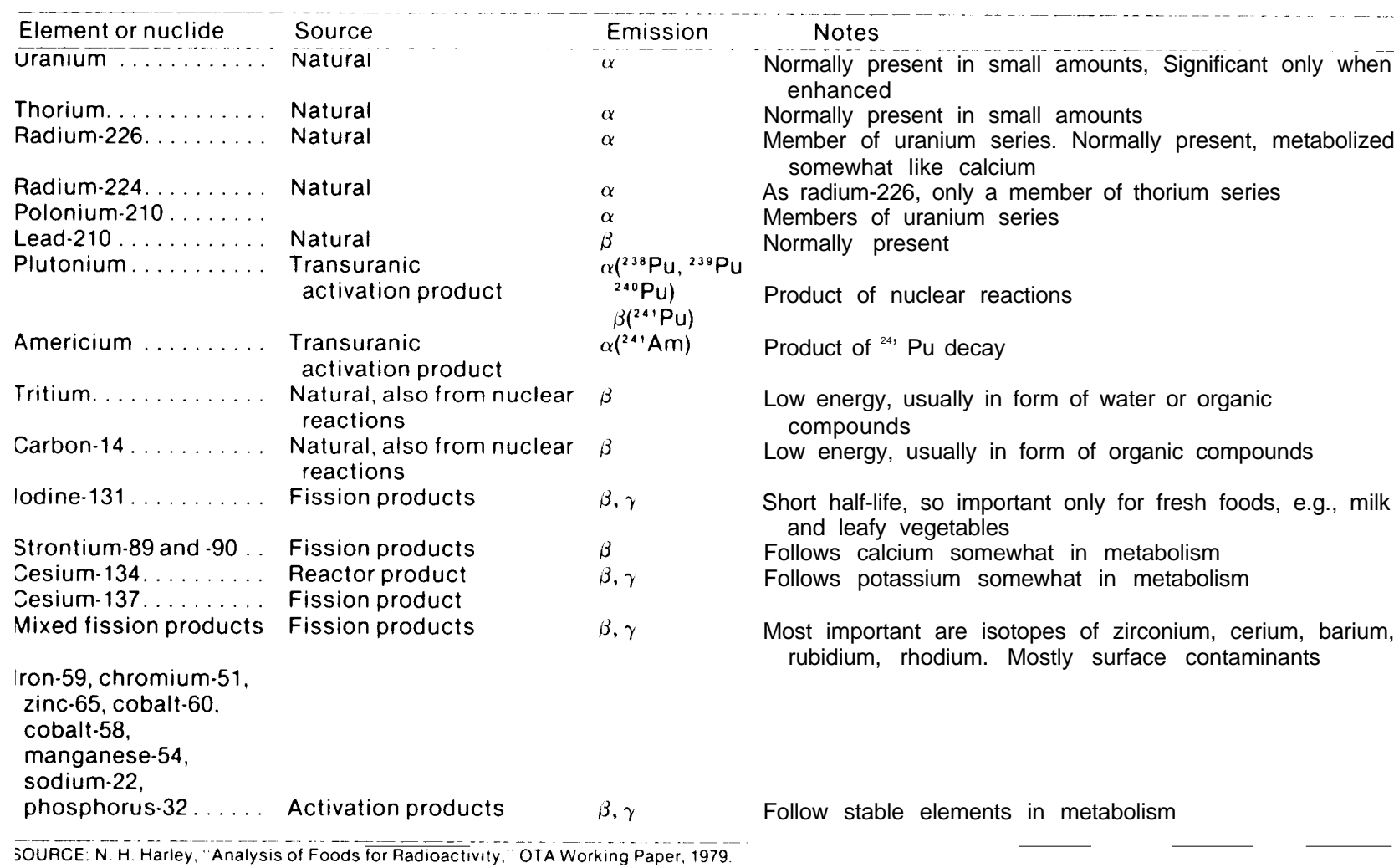




\section{MAGNITUDE OF THE PROBLEM}

There is little information available on the number of food contamination incidents, the amount and costs of food lost through regulatory actions, or the effects of consumption of contaminated food on health. To obtain information on the extent of the problem, OTA reviewed the literature and sought information from the States and Federal agencies.

\section{Evidence of Human IIIness Resulting From Consumption of Contaminated Food}

In evaluating the significance of environmental contaminants in food the key question is whether consumption of contaminated foods poses a health risk. Measurable health effects depend on the toxicity of the substance, the level at which it is present in food, the quantity of food consumed, and the vulnerability of the individual or population. In Japan, foods contaminated with substances such as PCBs, mercury, and cadmium have produced human illness and death. No such mass poisonings have occurred in the United States. However, in cases such as PBBs where a large populace has been exposed, some physiological changes have been noted. But no conclusions can as yet be drawn on the ultimate health effects.

It is known from limited surveys that the U.S. population is exposed to a wide variety of chemical contaminants through food, air, and water. The long-term health effects and the implications of possible interactions among these residues are unknown. A recent literature review of over 600 published studies (4) found that nonoccupationally exposed U.S. residents carry measurable residues of 94 chemical contaminants. Twentysix of these are organic substances, including twenty pesticides and pesticide metabolizes. The remainder are inorganic substances.

Americans also have been exposed to low levels of PCBs, PBBs, mercury, and ionizing radiation through their food. The following sections briefly summarize current knowl- edge and the extent of uncertainties on the health effects of these environmental contaminants.

\section{Polychlorinated Biphenyls}

PCBs occur in food as the result of environmental contamination leading to accumulation in the food chain, direct contact with food or animal feeds, or contact with foodpackaging materials made from recycled paper containing PCBs (5). Several comprehensive literature reviews have been published in the last 5 years detailing the acute and chronic toxic effects of PCBs in animals and humans (5-1 1).

Human illness has been caused by exposures to PCBs at much higher levels than those that occur in the United States. In the early part of 1968 the accidental contamination of edible rice-bran oil led to a poisoning epidemic among the Japanese families who consumed the oil. The disease became known as Yusho or rice-oil disease. Its chief symptoms were chloracne (a severe form of acne) and eye discharge; other symptoms included skin discoloration, headaches, fatigue, abdominal pain, menstrual changes, and liver disturbances. Babies born to mothers who consumed the rice oil were abnormally small and had temporary skin discoloration. The first symptoms of Yusho disease were registered on June 7,1968 , and 1,291 cases had been reported as of May 1975 (9).

Since the rice oil was also contaminated with polychlorinated dibenzofuran (PCDF), it is difficult to determine from the Yusho data exactly what effect(s) exposure to PCBs alone could have on humans. It has been calculated that the PCDF made the rice oil 2 to 3.5 times more toxic than would have been expected from its PCB content alone (1 1). Careful records of the 1,291 Yusho patients have been kept to determine possible long-term effects. At least 9 of 29 deaths that occurred as of May 1975 were attributed to cancer (malignant neoplasm), but a causal relationship be- 
tween PCBs and cancer cannot necessarily be inferred because of the high concentration of PCDF in the oil. The Yusho study, nevertheless, had two important results: first, the information established that PCBs can be transferred from mother to fetus and from mother to child through breast feeding, and second highly chlorinated PCB compounds are excreted more slowly from the body than less chlorinated ones (9).

More recent experiments in animals have demonstrated a variety of toxic effects. Cancers have been produced in mice and rats fed PCBs $(6,12)$. Monkeys fed levels of PCBs equivalent to the amounts consumed by $\mathrm{Yu}$ sho patients developed similar reproductive disorders (13-16). Young monkeys nursing on mothers consuming feed containing PCB developed toxic effects and behavioral abnormalities (15-1 7)

\section{Polybrominated Biphenyls}

Practically every Michigan resident has been exposed to PBB-contaminated food products. It is estimated that some 2,000 farm families who consumed products from their own PBB-contaminated farms have received the heaviest exposure (18).

Fries (19) studied the kinetics of PBB absorption in dairy cattle and its elimination in milk, If intake of contaminated milk alone is considered, those Michigan residents most severely exposed consumed from 5 to 15 grams of $\mathrm{PBB}$ over the initial 230 days of the exposure. Those residents that coincidentally consumed contaminated meat and/or eggs may have received higher total doses of PBB, but the number of such cases is probably small,

Geographically the residents of the lower peninsula, where the original accident occurred, were found to have the greatest levels of exposure. In 1976, the Michigan Department of Public Health conducted a study on PBB concentrations in breast milk. It was found that 96 percent of the 53 women selected from the lower peninsula and 43 percent of the 42 women selected from the upper peninsula excreted PBB in their breast milk (20).

Low concentrations of PBBs also have been detected in animal feed in Indiana and Illinois. Unconfirmed surveys of food throughout the country found extremely low levels below the Food and Drug Administration (FDA) action level in the following States (21):

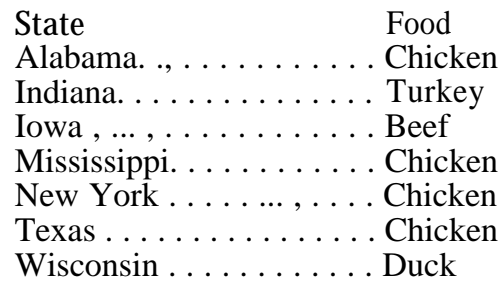

Wolff, et al. (22) reported that serum PBB was higher for males than females. It was suggested that the greater proportional body fat in women may account for this difference, but exposure may also be important. Males may consume more contaminated food or have more direct contact with $\mathrm{PBB}$ than females.

The same study found no consistent trends with respect to age. It was observed, however, that young males had greater concentrations of serum PBB than young females. Young females had greater concentrations than older males, and older males had greater concentrations than older females. It was also found that very young children and individuals who had lived on farms less than 1 year had lower serum PBB levels than other groups (22)

Serum PBB concentration is related to the intensity of exposure. Most studies indicate that consumers and residents of nonquarantined farms had significantly lower PBB levels than residents of quarantined farms; however, families on quarantined farms stopped consuming meat and milk from their own animals (20)

In late 1974, the Michigan Department of Public Health conducted a survey to determine if any adverse effects could be correlated with PBB levels in the body, A sample of 165 exposed persons (quarantined farms) 
and 133 nonexposed (nonquarantined farms) was studied. Medical history interviews and physical examinations were performed on each subject and blood specimens were taken, Blood PBB levels as high as 2.26 parts per million (ppm) were found in the exposed individuals; about half exhibited levels greater than $0.02 \mathrm{ppm}$. Of the nonexposed individuals, only two showed blood PBB levels greater than $0.02 \mathrm{ppm} ; 70$ percent of the adults and 97 percent of the children exhibited levels of 0.0002 to $0.019 \mathrm{ppm}$. Comparison of a list of selected conditions and complaints revealed no significant differences in the frequency of illness between the two groups. Physical examinations and clinical laboratory tests disclosed no effects attributable to "chronic" PBB exposure (24).

The effect of PBB exposure on white blood cell (lymphocyte) function of Michigan dairy farmers who consumed contaminated farm products was examined by Bekesi, et al. (25). Forty-five members of Michigan farm families who had eaten PBB-contaminated food for periods of 3 months up to 4 years after the original accident were compared for immunological function to 46 Wisconsin farmers and 79 New York residents. All of the exposed individuals showed reduced lymphocyte function, and 40 percent showed abnormal production of lymphocytes. There were also significant increases in lymphocytes with no detectable surface markers ("null" cells). However, the short- and long-term health implications of these differences are not now known.

Lillis (20) examined Michigan farmers and consumers of dairy products and found that the effect of PBB on humans was mainly neurological in nature. He found marked fatigue, hypersomnia, and decreased capacity for physical or mental work. Other symptoms included headache: dizziness: irritability; and musculoskeletal, arthritis-like complaintsswelling of the joints with deformity, pain, and limitation of movement. Less severe gastrointestinal and dermatological complaints were also encountered.

\section{Mercury and Methylmercury}

Foods are the major source of human exposure to mercury. The mercury concentration in food is dependent on the type of food, the environmental level of mercury in the area where the food is produced, and the use of mercury-containing compounds in the agricultural and industrial production of the food. All living organisms have the ability to concentrate mercury, Therefore, all animal and vegetable tissues contain at least trace amounts (26). Several recent reviews have examined the health effects associated with consumption of mercury (26-28). The results of these reviews indicate that the effects of methylmercury poisoning become detectable in the most sensitive adults at blood levels of mercury of 20 to $50 \mu \mathrm{g} / 100 \mathrm{ml}$, hair levels from 50 to $120 \mathrm{mg} / \mathrm{kg}$, and body burdens between 0.5 and $0.8 \mathrm{mg} / \mathrm{kg}$ body weight (26).

Since the Minamata Bay tragedy in Japan, the effects of chronic exposure to methylmercury have been well-documented. Mercury readily accumulates within the central nervous system (29-3 1), and clearance of mercury back into the bloodstream is slow (32). Consequently, the central nervous system is considered to be the critical target in chronic mercury exposure. The clinical symptoms of central nervous system involvement include headache, vertigo, vasomotor disturbance, ataxia, and pain and numbness in the extremities (30). The most prominent structural changes of the central nervous system resulting from chronic mercury exposure are diffuse cellular degeneration (30).

In evaluating the teratogenic hazards of mercury exposure to man, the placental transfer of mercury is particularly significant. Levels that are not toxic to pregnant women are sufficient to produce birth defects in their offspring (33-35). Transfer of methylmercury across the human placenta results in slightly higher blood levels in the infant at birth than in the mother (36). Table 5 compares fetal and maternal blood concentra- 
Table 5. - Methylmercury Concentrations in Normally Exposed Populations

\begin{tabular}{|c|c|c|c|c|}
\hline & & Concer & ntration $(\mu \mathrm{g}$ & $\mathrm{Hg} / \mathrm{g})$ \\
\hline Location & Maternal & blood & Placenta & Fetal blood \\
\hline Japan $\quad$...- & - ‘ 0.017 & & 0.072 & 0.020 \\
\hline Sweden ...... & . $\quad 0.00$ & & - & 0.008 \\
\hline Tennessee. . & 0.00 & & 0.021 & 0.011 \\
\hline lowa. . . . & 0.001 & & 0.002 & 0.001 \\
\hline
\end{tabular}

tions in normally exposed populations in Japan, Sweden, and the United States,

In humans, the most widely reported fetal risk associated with maternal exposure to mercury is brain damage. The placental transfer of mercury and its effects on the human fetus were first recognized in the 1950's with the well-known outbreak of congenital Minamata disease in the towns of Minamata and Niigata, Japan. By 1959, 23 infants suffering from mental retardation and motor disturbances had been born to mothers exposed to methylmercury during their pregnancies. The clinical symptoms of the infants resembled those of severe cerebral palsy or cerebral dysfunction syndrome. They included disturbance of coordination, speech, and hearing; constriction of visual field; impairment of chewing and swallowing; enhanced tendon reflex; pathological reflexes; involuntary movement; primitive reflexes; superficial sensation; salivation; and forced laughing (30). Only 1 of the 23 mothers exhibited any symptoms of mercury poisoning (32).

\section{Radioactivity}

Ionizing radiation (X-rays, gamma rays, or beta particles with sufficient energy to strip electrons from molecules and produce ions) can produce birth defects, mutations, and cancers (37). These adverse health effects are usually associated with high dose levels delivered at high dose rates.

Such a combination is not ordinarily encountered in food. Previous radioactive contamination of foods has involved relatively small quantities of radioactive elements which have delivered low dose rates (38).

In these situations, the effects of the radia- tion exposure on health are extremely difficult to evaluate. High dose rates (100 million to 1 billion times background) are estimated to produce 2,600 ionization events per second in cells. Background radiation levels are estimated to produce less than one ionization in the cell nucleus per day (37). Because cells have the capacity to repair damage to their genetic material, repair of ionization damage may occur at low radiation exposure. Higher exposures may overwhelm the cells' repair capacity. Whether any effects are observed in such cases depends on several factors. These include the dose delivered to the tissues, the nature of the emissions, and the metabolism of the cell. The following examples illustrate these points:

- Strontium-90 in food arouses most concern not only because of its long half-life but also because it behaves in the body in a manner somewhat similar to calcium. The replacement of bone calcium with strontium-go exposes tissues and cells covering the bone to radiation, In addition, bone marrow is subject to the ionizing radiation from the strontium-go. Thus, cancer of the bone-forming and bone-covering tissue as well as leukemias of the bone marrow blood-forming cells can possibly result.

- Iodine is concentrated by the thyroid gland. Radioiodines produced in atmospheric nuclear detonations or released from nuclear power stations are also taken up and concentrated by the thyroid, increasing the risk of thyroid cancer.

- Tritium, or radioactive hydrogen, combines chemically with oxygen to form water. Tritium derived from food would be widely distributed throughout the body exposing all tissues to radiation.

The uncertainties surrounding the repair capacities of cells and the irreversible nature of the possible health effects have led to the adoption in the United States of a prudent policy toward low-level ionizing radiation. Since any amount of radiation is potentially harmful, unnecessary exposure should be avoided. 


\section{Number of Food Contamination Incidents}

Questionnaires were mailed to the commissioners of health in each of the 50 States and the District of Columbia as well as to Federal agencies. For the 10-year period 1968-78, each was asked to report on the number of incidents of environmental contamination of food that resulted in regulatory action. This survey has limitations. Some States did not answer all questions. The questions were subject to interpretation and misunderstanding. The accuracy and completeness of the answers were dependent on the respondent. The results presented are therefore preliminary and do not necessarily represent complete and comprehensive information on all States responding. Nonetheless, these data are the first to be developed on the extent of environmental contamination of food.

Responses were received from 32 States. Seven of the top ten agricultural States and six of the top ten manufacturing States responded to the questionnaire, The agricultural States in the top 10 were California, Texas, Minnesota, Nebraska, Kansas, Indiana, and Missouri. The manufacturing States in the top 10 were California, New York, Michigan, New Jersey, Texas, and Indiana. Three of these States-California, Texas, and Indiana-are in the top ten for both agricultural and manufacturing production. A fairly representative distribution of States responded from each region of the United States (figure 2).

In the following discussions, an incident is defined as a case in which a Federal or State agency has taken regulatory action against contaminated food, The Michigan PBB episode is reported as one incident because the contamination stemmed from one source and was limited to one State. Mercury contamination is reported as separate incidents because the sources differed (environmental mercury v. industrial waste), the States involved are widely separated, and regulatory actions were taken at different times, Eighteen States reported at least one environmen- tal contaminant incident since 1968 for a total of 88 incidents. All food categories were involved and a variety of substances were implicated (see tables 1-3).

The data provided by States are complemented by the Federal responses. The two Federal agencies responsible for regulating the Nation's food supply reported the number of environmental contamination incidents that they had identified since 1968. FDA had 108 reported incidents, and the Food Safety and Quality Service of the U.S. Department of Agriculture (USDA) had 47 reported incidents (see table 3 ). The combined Federal and State total number of incidents comes to 243 .

Neither State nor Federal responses indicated any significant radionuclide contamination episodes during the 1968-78 period. Extensive Government programs for monitoring radionuclides in food exist. Thus far, radionuclide contamination of food has not been found to exceed the exposure limits recommended in the Federal Radiation Council Protective Action Guides. In most cases, the amount of food contamination in the continental United States has never even approached these limits (39), While atmospheric nuclear testing is less a threat today than before the signing of the 1963 Test Ban Treaty, radionuclide contamination of food is still a concern of both Federal and State governments.

The number of food contamination incidents reported to OTA does not represent the total number that has occurred in the United States, only those in which the Federal Government and 18 State governments have taken regulatory action. Many incidents never come to the attention of State or Federal authorities. This is because local government officials can and do handle environmental contaminant incidents by warning offenders or by condemning contaminated products without informing the appropriate State officials. Also, the farmer whose livestock or poultry has been environmentally contaminated may negotiate directly with the firm responsible for the contamination for financial 


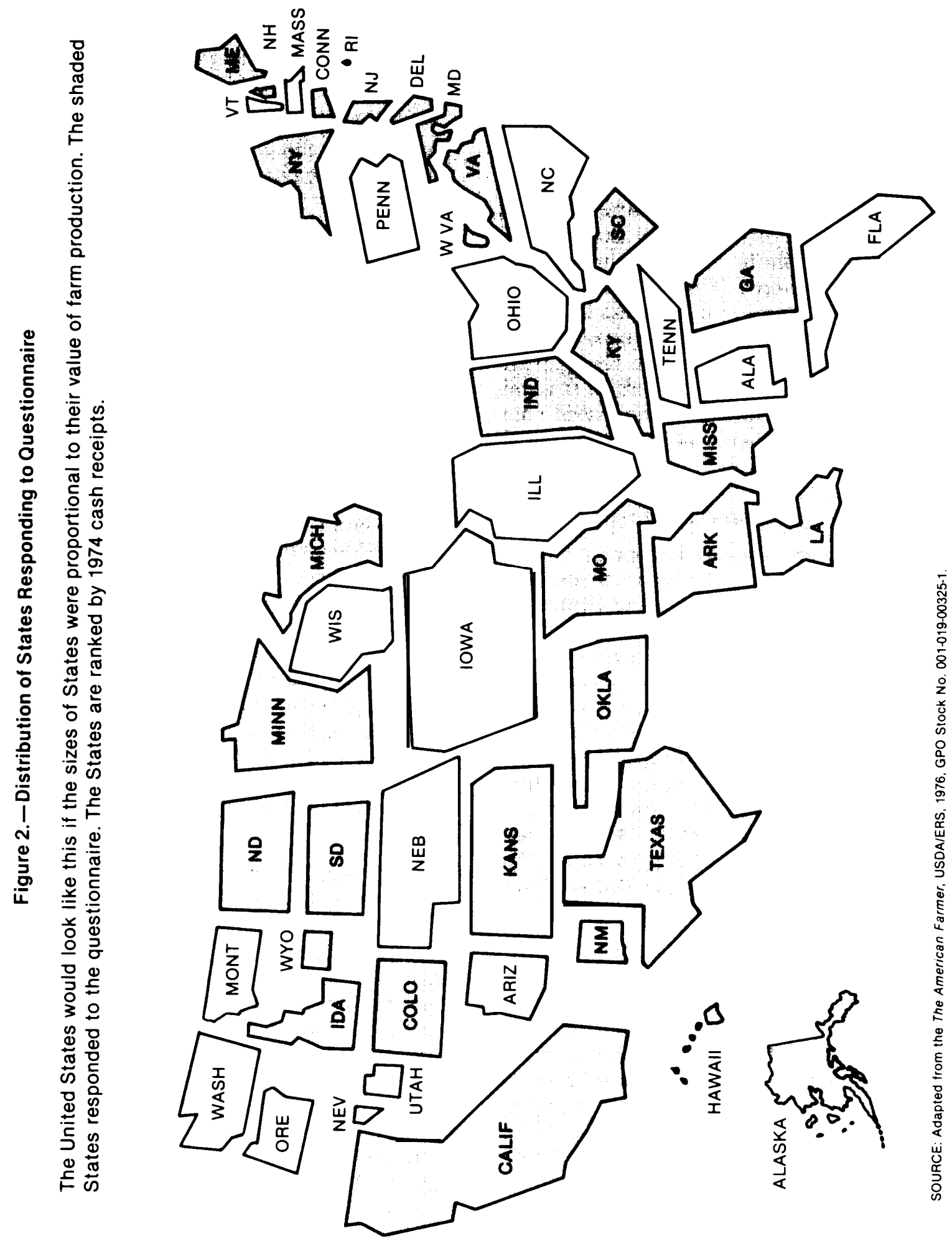


reimbursement without reporting the contamination to Federal or State officials (40).

\section{Economic Impact}

The economic impact of an incident involving the environmental contamination of food includes the cost of condemned food, health costs, and the corresponding distributional effects and costs. The magnitude of the economic impact is determined by:

- the amount of food contaminated,

- the concentration of the contaminant in food,

- the chemical and toxicological characteristics of the contaminant, and

- the corresponding regulatory action taken on the contaminated food.

The initial regulatory action taken by Federal and State authorities may be the issuance of a warning or the establishment of either an action level or a tolerance. A more detailed discussion of this regulatory action is presented in chapter III. Action levels and tolerances establish a permissible level for the contaminant in food. Any food found to contain concentrations of the substances above this level is condemned and either destroyed or restricted from being marketed.

\section{Costs of Food Condemned}

In addition to the four factors listed above, the cost of condemned food is also affected by its position in the food production and marketing process at the time of condemnation, An action level or tolerance for a contaminant is the most important of the five factors. If no action level or tolerance is set, no food would be condemned and thus there would be no costs incurred. The impact of such a regulation will depend on the exact level of a substance that is allowed to be present in food,

The chemical properties of a contaminant are also important because of the potential for long-term effects on the amount of food affected. Since many contaminants biologically and chemically degrade slowly, their pres- ence in the environment can mean food contamination above the action level or tolerance for many years after the source of the pollution has been stopped. The James River in Virginia, for example, is still closed to commercial fishing several years after kepone discharges into the river have been eliminated. The relative influence for each of these factors on the final cost will vary in each contamination incident.

Estimates of the cost of food condemned through regulatory action are most often expressed in dollars. Consequently, this cost is usually (and incorrectly) cited as representative of the total economic impact. Such costs were collected in OTA's State and Federal surveys. The data, however, only partially reflect the total economic impact for environmental contamination of food in the United States. This is because the cost of condemned food is only one component of the total economic impact of an incident. In addition, few of the incidents reported to OTA included data on the cost of food condemned. OTA estimates from the available data that the total cost of condemned food as a result of environmental contamination in the United States since 1968 is over $\$ 282$ million (table 6). The only cost estimates used were those clearly stated for an incident by the reporting States or Federal agencies.

State Estimates. - Of the 18 States reporting contamination incidents, only 6 provided data on the economic impact in dollar terms. Of those six, Michigan represents 99 percent of the total cost (\$255 million) while reporting only 19 percent of the number of incidents in the 18 States. Indeed, Michigan accounts for 90 percent of the total costs reported in the United States while reporting only 7 percent of the incidents that occurred during the 1968-78 period. It must be recognized, however, that 84 percent of Michigan's costs are attributed to the PBB incident. Many incidents reported by State and Federal agencies are considerably smaller than the PBB episode. Thus, the PBB episode is an indication of how severe a contamination incident can be. 
Table 6.-Economic Impact of Food Contamination

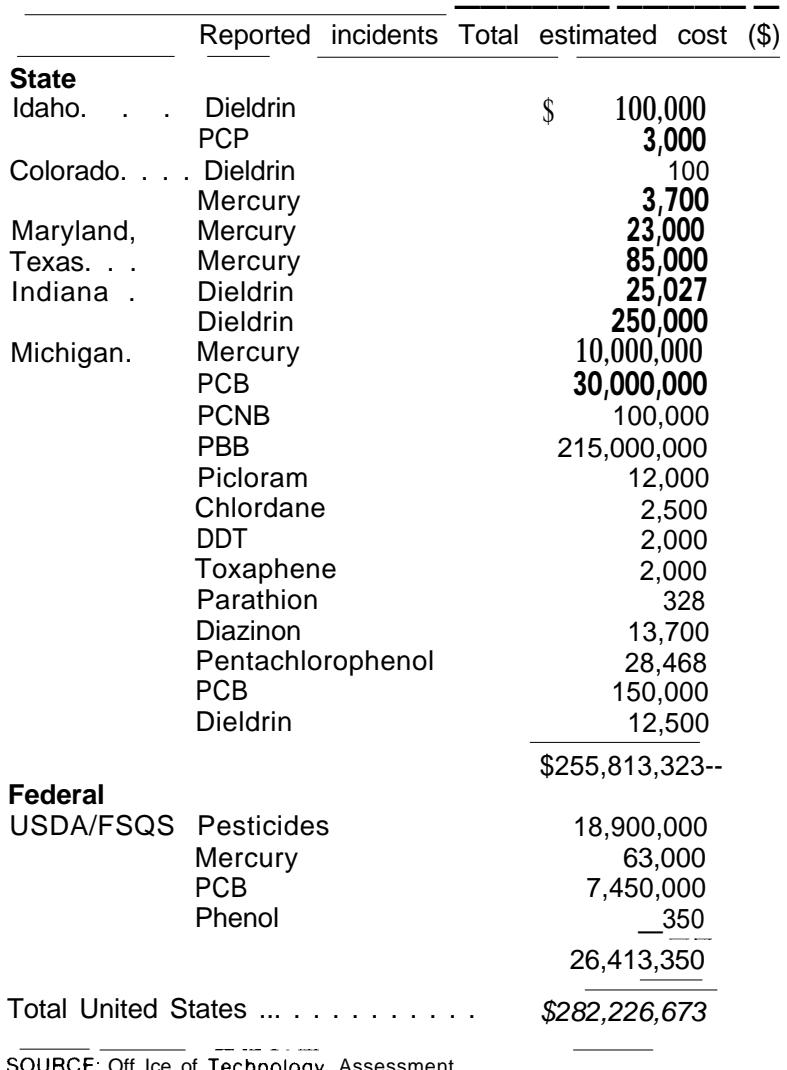

Some States reported the amount of food destroyed without estimating the cost. Kentucky, for example, reported the destruction of $\mathbf{4 0 0 , 0 0 0 ~} \mathrm{lbs}$ of milk since 1968 because of pesticide contamination. While such information can be converted into dollars, data on market position and price of product at time of confiscation are not readily available. Many States were unable to provide any estimates on either the cost or the amount of food condemned as a result of reported contamination incidents. New York (with PCBs) and Virginia (with kepone) are two States that could not provide cost estimates for food condemned as a result of environmental contamination. Virginia, however, has initiated a study to determine the economic impact of the kepone incident.

Federal Estimates.-Of the two Federal agencies reporting information to OTA on environmental contaminant incidents, USDA's
Food Safety and Quality Service (FSQS) reported food condemnation cost estimates. These estimates, however, only cover livestock and poultry - the food products over which FSQS has regulatory authority. FDA, which has regulatory authority over the remaining food commodities, did not estimate costs for reported environmental contamination incidents (70 percent of the Federal total). Thus, a significant proportion of the total costs for environmental contamination incidents requiring Federal action is unknown. Comparison of the two agency responses with the State responses reveals little duplication in the reporting of incidents.

FSQS cost estimates were determined by the number of animals or pounds destroyed multiplied by the market value at the time of confiscation. Since most of these animals were taken at the farm or wholesale level, the market value was the farm or wholesale price. Most of the losses resulting from FDA actions would be based on a wholesale or retail price because the seized products had advanced further in the marketing system. Therefore, their estimated costs would be greater than if they were seized at the production level (generally the case with FSQS seizures).

Summing up, the available data on the cost of condemned food is limited; consequently OTA's \$282 million condemned-food estimate is likely to be a gross underestimation of the actual costs. The true cost would be impossible to estimate from this limited sample.

\section{Health Costs}

Health costs are also an important component of economic impact. These costs are incurred by the consumer whose health has or potentially can be affected adversely by a contaminant present in food. These adverse effects can cause illness and death, and the range of effects will vary depending on the toxicity of the contaminant, the concentration of the contaminant in food, and the amount of food consumed. 
In this country, the concentration of contaminants has been at levels that have not produced immediate measurable and conclusive effects in exposed populations. Estimates are therefore made for the potential longterm effects on exposed populations from various contaminants in food.

Health costs can be estimated from such projected health effects. Costs would include health care costs for treating illness and burial expenses associated with death. Additional costs would include estimated value of productive days or years lost from work due to the projected illness or death associated with the contaminant in food. All of these health-related costs, however, do not and cannot include the emotional and psychological impacts on those afflicted and their friends and families.

Health costs are not available for previous U.S. food contamination incidents. Approaches and techniques for estimating health costs are discussed in chapter VI.

\section{Distributional Effects and Costs}

Distributional effects and costs involve the various people, groups, and organizations who are economically affected by an environmental contamination incident. Information on the extent and distribution of such effects and costs provides a clearer picture of the total economic impact on society. This information is usually couched in descriptive terms. Those who are economically affected are identified but the extent of the impact is seldom estimated in dollars. The exact distribution of costs from an incident through society is affected by the same five factors that influence the cost of condemned food.

Many of the distributional effects and costs for various types of environmental contaminant incidents are discussed in the following sections. The purpose of this discussion is not to identify all the distributional costs but rather to demonstrate the variety of effects and costs that can result from an incident.
Producers.- Food producers are affected economically in different ways by contamination episodes. But all are affected directly when the food they produce is condemned. For example, food found contaminated at the farm level is confiscated and destroyed. This was the case for over 500 Michigan farmers whose dairy herds were partially or entirely destroyed (41). In such cases, farmers either replace their livestock, plant a new crop, or go out of business.

Farmers can be faced with severe economic hardship, since they are not always reimbursed financially for the animals or commodities confiscated. While insurance programs such as the Federal Crop Insurance Corporation are available to cover natural hazards which might destroy crops or livestock, such Federal assistance is not available to farmers for losses from environmental contamination. An injured farmer can obtain a loan at commercial rates or sue the responsible firm for compensation. But the loan and the interest add to a farmer's financial difficulties, and suing for compensation can take time that the farmer may not have.

The commercial fisher is faced with a different situation. If a river, lake, or species of fish is restricted because of environmental contamination, the fisher whose source of income depends on this species or waterway may have few employment alternatives. The alternatives depend to some degree on the extent of the contamination. If the only waterway available in a section of a State or a whole State is closed to commercial fishing because of the contamination, the fisher's source of employment is eliminated until the restriction is ended. Since the restriction can last for years (depending on the chemical stability of the contaminant), the fisher either will have to move to other commercial fishing areas or seek other employment.

Food producers economically affected by the condemnation of contaminated food are likely to incur health costs. This is because many of the producers and their families regularly eat the food that they produce or har- 
vest. Consequently they are exposed to the contaminated food at greater concentrations than the average consumer. This was the case for several farm families in Michigan.

Firms Held Accountable for Environmental Contamination.-In most instances blame for a contamination incident can be established. Those accountable are subject to fines and lawsuits. Firms admitting responsibility often try to settle with producers out of court if possible. Most of the compensation is for the economic damages stemming from the destruction of food or loss of employment. Compensation for people whose health has been impaired as a result of eating contaminated food would be sought through civil litigation. Such litigation, however, is rare in this country, since the level of contamination in food is so low that demonstrating the necessary cause and effect is difficult.

Fines or compensation paid by the firms held accountable for the contamination are, in fact, poor indicators of the true costs incurred by the producers. This is because the settlement costs which are frequently negotiated or imposed bear little relationship to the actual costs incurred.

For example, compensation has been provided by Michigan Chemical Corporation and Farm Bureau Services, Inc., to many of the farmers whose livestock and poultry were destroyed following PBB contamination. Michigan Chemical and Farm Bureau Services have together paid more than $\$ 40$ million in compensation from a jointly established insurance pool (42). In another case involving PCB-contaminated fish meal sold to poultry producers, Ralston Purina Company negotiated compensation for the $\mathbf{4 0 0 , 0 0 0}$ chickens destroyed. The cost of the compensation has not been disclosed (43).

Governments.-Federal, State, and local governments also incur costs from an environmental contamination incident. Although the Federal Government and most State governments have agencies with programs to regulate or control food safety problems, these programs usually are not funded to handle the kind of long-term problems created by a PBB or kepone incident. The Michigan Department of Agriculture, for example, estimates it will spend $\$ \mathbf{4 0}$ million to $\$ 60$ million within the next 5 years to monitor and test for PBBs in animals and animal byproducts (44). This is money that could have been saved or spent for other programs if PBB contamination had not occurred. In order to recover its expenses from the PBB incident, the State of Michigan filed a lawsuit against both the Michigan Chemical Corporation and Farm Bureau Services, Inc., claiming more than $\$ 100$ million in damage (45).

Federal involvement is limited unless the contaminated food is part of interstate commerce, Many of these incidents are not considered by the Federal Government to involve interstate commerce, FDA may provide technical assistance at the request of the State government when a contamination incident is regarded to be a local problem (43). These technical facilities and experts are available to all States through the Federal and regional offices. Additional expenditures by the Federal Government for contamination incidents are limited. Additional State expenditures, however, can be substantial. Federal expenditures are made when Federal regulations are developed and promulgated for particular contaminants in food such as PCB.

Consumers.-Consumers can incur costs from an environmental contaminant incident in several ways. The removal of food from commerce could increase prices for that food product or other food products being sold. Thus, the consumer could pay more for food as a result of an environmental contamination incident. In order for this price increase in food to occur, however, a significant amount of a food product or food products would have to be taken off the market. Such prices of food might vary by State or region and affect certain socioeconomic classes differently.

Health costs could increase as a result of the consumption of contaminated food. This would not affect all consumers but rather those who received the most exposure and/or 
those most susceptible to a contaminant, such as children or senior citizens. While these costs would already be included in estimated total health costs, the distributional effects could indicate those consumers most likely to be affected.

Indirect Costs. - Most of the costs mentioned directly stem from an environmental contamination incident. However, indirect or secondary costs can and do occur. For exam- ple, a bait and tackle store on a lake that is closed to commercial and sport fishing because of an environmental contamination is likely to suffer economic hardship, Food processors whose normal supply of food has been condemned because of environmental contamination will also suffer economically unless they find new sources of supply. These are just two examples of the many indirect costs which might occur.

\section{POTENTIAL FOOD CONTAMINATION PROBLEMS}

Because a limited number of substances posing health problems already have been identified in food, concern exists that other toxic substances are likely to contaminate food in the future. This concern arises from the number of substances presently being manufactured, used, and disposed of in the United States, and the difficulties in preventing them from entering the environment. New substances developed to meet new needs or to replace known toxic substances may create unexpected environmental problems if not properly controlled. Byproducts of new technologies such as synthetic fuels are also potential environmental contaminants. These are described in appendix A.

There are two methods of objectively assessing possible future contaminants: 1 ) by sampling the food supply for chemical contaminants and ranking them according to po- tential hazard and 2) by surveying the universe of industrial chemicals and ranking them according to their potential for entering the food supply in toxic amounts. These methods are discussed in more detail in chapter VII, "Monitoring Strategies.

Of the three categories of environmental contaminants considered in this report, organic chemicals probably pose the greatest potential environmental and food contamination problems. This conclusion is based on the number, volume, and toxicity of the organics manufactured and used in this country (40). Both trace metals and radioactive substances continue to warrant concern, but not as great a concern as organic substances. The extent of food contamination from these substances depends on our success in preventing them from entering the environment.

\section{CONCLUSIONS AND ISSUES}

Data presented here indicate that environmental contamination of food is a nationwide problem of unknown magnitude. Long-term, low-level exposure to toxic substances in food poses health risks that are difficult to evaluate given present techniques. Incidents of high-level contamination of food that cause human illness have not occurred in the United States, However, regulatory actions have been taken to restrict consumption of contaminated food in cases where the potential health risks were considered unacceptable. These episodes have resulted in economic losses when contaminated food was removed from the market,

The following chapters analyze several issues related to the regulation of environmental contaminants in food. These are:

- Is our present regulatory system protecting the public health? (Chapters 111 and IV) 
- Are methods used by the regulatory agencies for estimating health impacts the most appropriate ones? (Chapters III and V)

- Should economic impacts be an explicit part of regulatory decisionmaking? If so, how should economic impacts be evaluated? (Chapters III and VI)

- Should regulatory monitoring be capable of detecting substances as they enter the food chain? (Chapters VII and VIII)

\section{CHAPTER II REFERENCES}

1. Storclz, William J. “C\&EN's Top Fifty Chemical Products and Producers, Chemical and Engineering News 56(18):33, 1978,

2. Murray, Chris, "Chemical Waste Disposal A Costly Problem," Chemical and Engineering News, p. 12, Mar, 12, 1979.

3. National Academy of Sciences, National Research Council, Commission on Natural Resources, Environmental Studies Board, Committee on the Assessment of Polychlorinated Biphenyls in the Environment. Polychlorinated Biphenyls (ISBN O-309-02885-X), 1979,

4. Hammons, A. "Levels of Chemical Contaminants in Nonoccupationally Exposed U.S. Residents," draft manuscript prepared for Health Effects Research Laboratory, Environmental Protection Agency, 1978.

5. U.S. Food and Drug Administration. "Pol chlorinated Biphenyls (PCBS), Federal Register 42(63):17487, 1977,

6. Kimbrough, R. D. "The Toxicity of Polychlorinated Polycyclic Compounds and Related Chemicals, "CRC Critical Reviews in Toxicology 2:445, 1974,

7, Fishbein, L, "Toxicity of Chlorinated Biphenyls," Ann. Rev. Pharmacol.14:139, 1974.

8. Peakall, D. B. "PCBs and Their Environmental Effects, CRC Critical Reviews on Environmental Contaminants 5:469, 1975.

9. National Institute of Occupational Safety and Health. Criteria for a Recommended Standard. Occupational Exposure to Polychlorinated Biphenyls (PCBs), 1977 (DHEW Publ. No, 77-225).

10. Kimbrough, R, D., et al. "Animal Toxicity," Environ, Health Persp. 24:173, 1978.

11. Cordle, F., et al. "Human Exposure to Polychlorinated Biphenyls and Polybrominated Biphenyls," Environ. Health Persp. 24:157, 1978.

12. Kimbrough, R. D., and R. E. Linder. "Induction of Adenofibrosis and Hepatomas of the Liver in BALB/oJ Mice by Polychlorinated Bi- phenyls (Aroclor 1254), "Journal of The National Cancer Institute 53:547, 1974.

13. Allen, J. R., et al. "Residual Effects of Short-Term, Low-Level Exposure of Non-Human Primates to Polychlorinated Biphenyls," Toxicology and Applied Pharmacology 30:440, 1974.

14. McNulty, W. P. "Primate Study," National Conference on Polychlorinated Biphenyls, Chicago, 1975.

15. Barsotti, D. A., et al. "Reproductive Dysfunction in Rhesus Monkeys Exposed to Low Leve $1 \mathrm{~s}$ of Polychlorinated Biphenyls (Aroclor 1248), " Food and Cosmetics Toxicology 14:99, 1976.

16. Allen, J. R., and D. A.Barsotti. "The Effects of Transplacental and Mammary Movement of PCBS on Infant Rhesus Monkey s," Toxicology 6:331, 1976.

17. Allen, J.R. "Response of the Non-Human Primate to Polychlorinated Biphenyl Exposure," Federation Proceedings 34:1675, 1975.

18. Meester, W. D. and D. J. McCoy, Sr. "Human Toxicology of Polybrominated Biphenyls, " Clinical Toxicology 10(4):474,1977.

19. Fries, G. F., G. S. Marrow, and R. M. Cook. "Distribution and Kinetics of PBB Residues in Cattle," Environmental Health Perspectives 23:43, 1978.

20, Lillis, R., H. A. Anderson, J. A. Valcinkas, S. Freedman, and I. J. Selikoff. "Comparison of Findings Among Residents of Michigan Dairy Farms and Consumers of Produce Purchased From These Farms," Environmental Health Perspectives 23:105, 1978.

21. Environmental Protection Agency, Office of Toxic Substances, Special Actions Group. "Assessment of the Hazards of Polybrominated Biphenyls (PBBs)" (draft), 1977.

22. Wolff, M. S., B. Aubrey, F. Camper, and N. Hames. "Relation of DDE and PBB Serum Levels in Farm Residents, Consumers, and Michi- 
gan Chemical Corporation Employ ees, "Environmental Health Perspectives 23: 177, 1978.

23. Cook, H. D., R. Helland, B. H. Vander Weele, and R. J.DeJong. "Histotoxic Effects of Polybrominated Biphenyls in Michigan Dairy Cattle,", Environmental Research 15:82, 1978.

24. Kay, K. "Polybrominated Biphenyls (PBB) Environmental Contaminants in Michigan, 19731976, "Environmental Research 13:74, 1977.

25. Bekesi, J. G., J. F. Holland, H. A. Anderson, A. S. Fischbein, W. Rem, M. S. Wolff, and I. J. Selikoff. "Lymphocyte Function of Michigan Dairy Farmers Exposed to Polybrominated Biphenyls," Science 199:1207, 1978.

26. United Nations Environment Program and the World Health organization. Environmental Health Criteria 1: Mercury, Geneva, p. 23, 1976 ,

27. National Academy of Sciences, National Research Council, An Assessment of Mercury in the Environment, Washington, D, C., 1978.

28. U.S. Environmental Protection Agency, Ambien t Water Quality Criteria: Mercury, 1979.

29. Lu, F. C. "'Mercury as a Food Contaminant, " WHO Chronicle 28:8, 1974.

30, D’Itri, F, M. “The Environmental Mercury Problem, " CRC Press, p. 73, 1972.

31. Friberg, L., and J. Vestal. "Mercury in the Environment, " Cl? Caress, 1972.

32. Gerstner, H.B., and J. E. Huff. '*Clinical Toxicology of Mercury, " Journal of Toxicology and Environmental Health 2:49 1,1977.

33. Olson, F. C., and E. J, Massaro. "Pharmacod namics and Tera tologic Effects of Transplacentally Transported Methyl Mercury in the Mouse," International Conference on Heavy Metals in the Environment, pp. 32 and 184, 1972.

34. Weiss, B., and R. H. Doherty. "Methylmercury Poisoning,"' Teratology 12(3):311, 1976.
35. Rizzo, A. M., and A. Furst. "Mercury Teratogenesis in the Rat, "Proceedings of the Western Pharmacology Society 15:52, 1972.

36. Evans, H. L., R. H. Garman, and B. Weiss. "Methylmercury: Exposure Duration and Regional Distribution as Determinants of Neurotoxicity in Nonhuman Primates, "Toxicology and Applied Pharmacology 41:15, 1977.

37. National Academy of Sciences, Advisory Committee on the Biological Effects of Ionizing Radiations. Effects on Populations of Exposure to Low Levels of Ionizing Radiation, Washington, D. C., 1972.

38. National Academy of Sciences, Food Protection Committee. Radionuclides in Foods, Washington, D. C., 1973.

39. Dodge, Chris. "Contamination of Food by Radioactive Nuclides," Congressional Research Service, OTA Working Paper, August 1978.

40. Monitoring Advisory Panel, Sept. 21, 1978.

41. Michigan Department of Agriculture, Information and Education Division. "A Brief Chronology of the PBB Incident" (no date).

42 Anonymous. "Two Firms Fined \$4,000 Each for PBB Contamination of Cattle Feed in Michigan, "Toxic Material News, p. 144, May 24, 1978,

43. Jaroslovsky, Rich. "Contamination of Fishmeal with PCBS Sparks FDA Study of Mishap at a Ralston Purina Plant, "The Wall Street Journal, p. 46, July 12, 1978.

44. Whitehead, George, Deputy Director for Consumer Affairs, Michigan Department of Agriculture, Jan. 16, 1978.

45 McNally, JoAnn. "Polybrominated Biphenyls: Environmental Contamination of Food, "Congressional Research Service, OTA Working Paper, Sept. 21, 1978. 
Chapter III

\section{Federal Laws, Regulations, and Programs}




\section{Federal Laws, Regulations, and Programs}

\section{FEDERAL LAWS}

Congress has enacted several laws that not only regulate but also attempt to limit or restrict the introduction of toxic substances into the environment. Table 7 summarizes the Federal laws affecting toxic substances control.

Some of these laws give Federal agencies authority to prevent unsafe food from reaching consumers. Most important in terms of this assessment is the Federal Food, Drug, and Cosmetic (FD\&C) Act (1). Broadly speaking, this statute prohibits the introduction of adulterated food into interstate commerce. The FD\&C Act allows the Food and Drug Administration (FDA) to establish tolerances for toxic substances whose occurrence in food cannot be avoided. The Poultry and Poultry Products Inspection Act (2) and the Federal Meat Inspection Act (3) give the U.S. Department of Agriculture (USDA) authority to inspect meat, poultry, and their byproducts. The adulteration provisions of these Acts govern all environmental contaminants except for pesticides that may occur in meat and poultry. Under these laws, section 408 of the FD\&C Act applies to such pesticide contamination. In practice, USDA uses the tolerances established under the FD\&C Act and consults with FDA and the Environmental Protection Agency (EPA) to determine an action level when no tolerance exists.

Following is a brief summary of the pertinent provisions of the FD\&C Act:

Section 402(a)(1) declares that any food that "bears or contains any poisonous or deleterious substance which may render it injurious to health" is adulterated. The single exception is if the substance is not, in the language of the Act, "added" to the food. In such cases, the presence of the substance does not imply that the food is adulterated unless it is present in sufficient quantity to "ordinarily render it injurious to health."

The FD\&C Act recognizes that certain "added" toxic substances in foods require special attention, Section 406 empowers FDA to establish tolerances for "added" poisonous substances whose occurrence in food cannot be avoided or whose use is "necessary" to produce the food. Thus, Congress authorized FDA to "license" the presence of certain potentially toxic substances in food, seeming- ly because of their economic utility or the inability of existing, commonly used production methods to eliminate them. The legislative history of section 406 is skimpy. But Congress' principal objective apparently was to permit continued use of pesticides on raw agricultural commodities while giving FDA an effective means of control-the power to declare illegal any food that contained any amount of an added substance that exceeds FDA tolerance. Congress left the distinction between "added"' and other constituents undefined, and did not attempt to clarify the concepts of "necessary" or "unavoidable"' in section 406(4).

Amendments to the 1938 FD\&C Act deal with specific categories within the broad class of substances "added"' to foods including pesticides, food additives, vitamins and minerals, and animal drugs. Each amendment in effect establishes a system under 
Table 7.-Federal Laws and Agencies Affecting Toxic Substances Control

Statute
Toxic Substances enacted

Clean Air Act. . . . . . . . . . . . . . . . . 1970, amended 1977

Federal Water Pollution Control Act

(now Clean Water Act)

1972, amended 1977

Safe Drinking Water Act ...........1974, amended 1977

Federal Insecticide, Fungicide, and 1947, amended 1972, 1975, EPA

Rodenticide Act . . . . . . . . . . . 1978

Act of July 22, 1954, (codified as

Section 346(a) of the Food, Drug, 1954, amended 1972

and Cosmetic Act)

Resource Conservation and

Recovery Act . ..............1976

Marine Protection, Research, and

Sanctuaries Act . . . . ..........1972

Food, Drug, and Cosmetic Act ......1938

Food additives amendment . .....1958

Color additives amendments .... 1960

New drug amendments . . . . . . . . . 1962

New animal drug amendments. .. .1968

Medical device amendments .. 1976

Federal Meat Inspection Act. . ......1967

Poultry Products Inspection Act .... .1957

Egg Products Inspection Act . ......1970

Fair Packaging and Labeling Act .. ...1976

Public Health Service Act ... . .....1944

Occupational Safety and Health Act .1970

Federal Hazardous Substances Act. 1960

Consumer Product Safety Act 1972

Poison Prevention Packaging Act .. 1970 $\begin{array}{lll}\begin{array}{l}\text { Lead-Based Paint } \\ \text { Prevention }\end{array} & \begin{array}{c}\text { Poisoning } \\ \text { Act }\end{array} & \text { 1973, amended } 1976 \\ \begin{array}{c}\text { Hazardous Materials Transportation } \\ \text { Act }\end{array} & 1975 \text {, amended } 1976\end{array}$

Federal Railroad Safety Act . . . . 1970

Ports and Waterways Safety Act 1972

Dangerous Cargo Act .. ...1952

Federal Mine Safety and Health Act. .1977

CPSC $=$ Consumer Product Safety Commission

DOT $=$ Department of Transportation

$\mathrm{EPA}=$ Environmental Protection Agency

FDA = Food and Drug Administration

$\mathrm{HEW}=$ Health Education and Welfare

EPA

EPA

EPA

EPA

EPA

EPA

FDA

FDA

FDA

FDA

FDA

FDA

USDA

USDA

USDA

FDA

FDA

OSHA, NIOSH

CPSC

CPSC

CPSC

CPSC, HEW, HUD

DOT (Materials

Transportation

Bureau)

DOT (Federal

Railroad Admin.)

DOT (Coast Guard)

Labor (Mine Safety .), physical agents in coal or other $\mathrm{NIOSH}$

HUD $=$ Housing and Urban Development

$\mathrm{NIOSH}=$ National Institute for Occupational Safety and Health

OSHA = Occupational Safety and Health Administration

USDA = United States Department of Agriculture

SOURCE Environmental Law Institute, An Analysis 01 PastFederalEfforts To ControlToxic Substances Washington D C 1978 
which FDA is empowered to license, and thereby limit, the use (or in the case of pesticide residues, the occurrence) of potentially toxic substances in or on food.

The Pesticide Chemicals Amendment of 1954, now section 408 of the FD\&C Act, provides that a raw agricultural commodity shall be deemed to be adulterated if it bears or contains any residue of a pesticide that does not conform to a tolerance established under section 408, Pesticides that are unintentionally present on commodities are usually considered environmental contaminants by FDA, and are regulated under section 406.

At no point, in 1938 or subsequently, has Congress specifically addressed the problem of environmental contaminants in food. FDA could have regulated them all under the "may render injurious" language of section 402(a) (1). But this provision would not have given FDA authority to determine administratively what levels of a contaminant could be tolerated. FDA would have been required to prove its claim of hazard each time it seized a contaminated product.

Accordingly, since the early 1970's FDA has classified environmental contaminants as "added poisonous or deleterious substances' whose occurrence cannot entirely be avoided, thus avoiding the less rigorous "ordinarily injurious" standard of section 402(a) (1). The tolerance-setting authority of section 406 can then be applied to environmental contaminants in food.

\section{ACTION LEVELS AND TOLERANCES}

Relying on section 406, FDA prescribes the level of a contaminant that, under section 402(a)(2)(A), will render a food adulterated. Before FDA can ascertain this level, sufficient scientific data must be accumulated to answer several questions implicitly posed by sections 402 and 406. FDA must be able to determine that the environmental contaminant in question is:

1. added,

2. poisonous or deleterious,

3. a substance unavoidable by good manufacturing practice, and

4. one which may make the food injurious to health.

Furthermore, an analytical method that can reliably detect, measure, and confirm the identity of the contaminant in the food under scrutiny must be available (5).

To determine whether these requirements can be met, FDA scientists explore and review the scientific literature, consult FDA files, and draw on information available in other agencies, in academia, or in private industry. Then, based on the best scientific data available (which are often incomplete), FDA will prescribe what level of contamination will trigger enforcement action (5).

Regulatory procedures employed to control environmental contaminants in food include the establishment of action levels or tolerances. A formal tolerance is a regulation having the force of law. Tolerances are adopted through formal rulemaking procedures and specify the level of a contaminant that will render a food adulterated. If supported by substantial evidence in the rulemaking record, FDA's tolerance cannot be questioned by any court. An action level is an in.formed judgment about the level of a food contaminant to which consumers may safely be exposed. It is a statement of FDA's professional judgment and represents a commitment to initiate regulatory enforcement action against any lots of food discovered containing excess levels. Essentially the same criteria are considered in establishing tolerances and setting action levels. The principal differences between the two approaches lie in the procedures for their adoption, the strength of the scientific data supporting them, and the differing weight they carry in court (4). 
FDA will set a tolerance when the following conditions exist:

1. The substance cannot be avoided by good manufacturing practice.

2. The tolerance established is sufficient for the protection of the public health, taking into account the extent to which the presence of the substance cannot be avoided and the other ways in which the consumer may be affected by the same or related poisonous or deleterious substances.

3. No technological or other changes are foreseeable in the near future that might affect the appropriateness of the tolerance established (6).

To establish a tolerance, FDA first publishes a proposal, accepts comments, and issues a "final"* regulation, Formal objections can be raised to this "final" tolerance. Such objections can, if they raise material issues of fact, require a lengthy trial-type hearing before an FDA administrative law judge, who then issues an initial decision based on the formal hearing record. That decision, in turn, can be appealed to the FDA Commissioner (who issued the original "final" tolerance). The Commissioner's ultimate decision is subject to review in a court of appeals (1).

Because the tolerance-setting procedure is cumbersome and time-consuming, FDA initially relies on an action level when it regulates an environmental contaminant. An action level is an administrative guideline and the functional, though not legal, equivalent of a section 406 tolerance. It is established when "technological or other changes that might affect the appropriateness of the tolerance are foreseeable in the near future" (6).

To set an action level, FDA simply announces in the Federal Register that it is establishing an action level for a contaminant, and states that the data supporting the designated level are available for public inspection. This announcement may briefly discuss the pertinent factors that went into the decision, but any discussion is not likely to be (nor is required to be) extensive. While the announcement also notes that public com- ments will be accepted, FDA makes no commitment to respond to any comments or, indeed, to reconsider the action level within any specified period (6). The process does not require a detailed public discussion of the selected levels, nor does it trigger a public debate about the correctness of FDA's premises or its balancing of relevant factors.

Finally, the Commissioner of FDA may exempt from regulatory action any contaminated food if he determines "based upon all available scientific evidence, that the food is safe for consumption and that destruction or diversion of the food involved would result in a substantial adverse impact on the national food supply" (6). This has only happened once, and the action did not involve human food,

If the environmental contaminant is a pesticide for which no tolerance has been established by EPA, FDA relies on EPA to recommend an action level (as it did in the case of kepone). In other respects, the procedures and criteria for regulating pesticides as environmental contaminants are the same as for other contaminants.

\section{Criteria for Setting Action Levels and Tolerances}

For the setting of action levels or tolerances, neither the law nor regulations require FDA or EPA to follow a standardized set of toxicologic protocols to evaluate risk. No policy exists defining the relative weight to be given to evidence. The burden of proving there is a health hazard lies with FDA. When setting an action level or tolerance, FDA considers the following types of data:

1. available acute and chronic toxicological data, including information on the biological half-life of the substance and its metabolic fate;

2. available data on the levels and incidence of the contaminant in the overall food supply and specifically in the food commodity or commodities that are being considered for an action level or tolerance; 
3. normal serving sizes of the concerned food(s) and frequency of ingestion;

4. susceptibility of certain population groups, such as infants and the aged, to adverse effects from anticipated dietary exposure to the contaminant:

5. the level at which available analytical techniques can detect, measure, and confirm the identity of the contaminant;

6. capability of manufacturers to monitor their food production to ensure that the products comply with the action level or tolerance; and

7. the anticipated impact of various possible levels of regulation on the national food supply.

In response to questions on how FDA evaluates these data, Commissioner Donald Kennedy wrote:

Each factor is assessed individually (assuming information on each is available) and then collectively brought into balance by a composite analysis in terms of the estimated risk to the public health versus both the extent to which the substance is unavoidable and the quantity of food that would be unlawful under levels being considered (5),

FDA has not fixed the weight to be given to each of the above factors. Each will, to some degree, influence the final decision; generally, the more information about a particular factor, the greater its influence, This is one reason that FDA offers for not prescribing a predetermined quantifiable set of criteria for each factor. The amount and quality of information available when FDA encounters an environmental food contamination problem are inevitably unpredictable, FDA maintains that because of this uncertainty, it is impractical to state in advance the precise weight of each factor in the final determination. However, FDA maintains that the public health factor outweighs all others in its considerations (5).

\section{Determining Action Levels and Tolerances}

In general, EPA and FDA follow similar procedures when evaluating the health risk associated with consumption of a toxic substance in food, Both agencies consider three areas when evaluating the scientific information: 1) existing animal toxicity data, 2) existing human toxicity data, and 3) exposure data based on the level of the contaminant in food and the average consumption of that food. Both agencies also consider what effect an action level or tolerance will have on the availability of food.

\section{Evaluation of the Scientific Data}

When a food contaminant is identified, the first step in establishing an action level or tolerance is to assemble and evaluate all available information on its toxicity. This information comes from articles published in the scientific literature, information provided by private industry, and data from other Government agencies. From animal toxicity data and whatever human toxicity data may be available, a no observed effect level (NOEL) is calculated and expressed in milligrams per kilogram of body weight per day. NOEL is the level at which the substance had no observed effects.

An acceptable daily intake (ADI) is then calculated by dividing the NOEL by a safety factor. The term "acceptable" does not imply absolute safety for all people in all cases, and the term "safety factor" implies more than it seems. The safety factor reflects the uncertainty of translating animal data to humans, the variability of the human population, the insufficiency of the data available, and the severity and reversibility of toxic effects.

When 2-year chronic toxicity studies in animals are available, the safety factor used is 100. When threshold levels have been observed in humans, the safety factor employed is 10. If long-term studies are available and show no irreversible effects, a much smaller safety factor might be selected. If evaluation of available toxicological data indicates that no threshold exists, a very large safety factor (on the order of 1,000 or more) maybe used. F. 'I'. Arnold, chairman of the Kepone Action Level Hearings, stated that "the determination of an appropriate safety factor is an art 
rather than a science and is dictated by the chemical in question, its toxicological properties and surrounding circumstances" (7).

The next step is to calculate the maximum permissible intake. The maximum permissible intake is the product of ADI and the average weight of an adult (this figure varies from 60 to $70 \mathrm{~kg}$ ). This figure is then compared to the maximum potential exposure through consumption of contaminated food. Tolerances are set so that the amount of the contaminant consumed in food is less than or equal to the ADI. Mathematically, this can be expressed as:

$$
\begin{aligned}
& \text { ADI } x \text { Average body weight } \\
& \text { Tolerance }= \\
& \text { of consumer } \\
& \text { Food factor } \times 1.5 \mathbf{~ k ~ g} \\
& \text { Where } A D I=\text { the NOEL divided by an appropriate }
\end{aligned}
$$

Appendix B provides a detailed example of how these concepts were applied by FDA in the development of tolerances for polychlorinated biphenyls (PCBs) in food,

These procedures have several limitations. The data on toxicity of an environmental contaminant for humans and animals may often be inadequate for setting a tolerance. Because of time constraints and the necessity to make a regulatory decision, action levels for environmental contaminants in food may be based on incomplete, scanty toxicological information. Once a level is set, however, no law requires FDA to collect new evidence on the toxicity of the substance in question even though that issue may well continue to be of critical importance to the population receiving the highest exposure and to food producers.

Because of the nature of the problem presented by environmental contaminants in food and because so little data are usually available, EPA and FDA cannot have formal requirements for toxicity data. Moreover, in most instances there are no petitioners or sponsors of commercial uses of the material to which FDA can look for the necessary additional tests. Available industry data are frequently used because of the lack of published or publicly available information on the substance. While known toxic effects of metabolic products of the substance in question are considered, unknown metabolizes cannot be. Finally, additive and synergistic effects between the contaminant and other toxic substances in food are not considered in the tolerance-setting procedures.

Questions are frequently raised about safety factors and the extrapolation from animal data to humans. Comparisons between animals and humans are based on milligrams of the toxic substance per kilogram of body weight, although many maintain that milligram of toxic substance per square centimeter of body surface area is a more appropriate comparison,

Little scientific evidence exists to support safety factors. Historically, a factor of 10 in extrapolating from animals to man and a factor of 10 in extrapolating from the least-sensitive human to the most-sensitive have been used although they have little theoretical or factual basis. Many believe that the use of different safety factors for different toxicological effects (a greater safety factor for irreversible effects, a smaller factor for reversible effects) or the use of mathematical models to extrapolate from animal to human risk would be more appropriate. Finally, the safety factor approach may not make allowances for vulnerable groups such as infants, except in those instances in which infants are considered the primary population at risk. Individuals with predisposing conditions or previous exposure may not be adequately covered by safety factors. In truth, it may be impossible to protect every individual with allergies or predisposing physiological conditions.

The methods used for estimating dietary exposure to toxic substances are limited by lack of sound data. FDA bases some tolerance decisions on their Total Diet Studies, Because 
these are based on the diet of a teenage male, they do not reflect the dietary patterns of vulnerable groups, nor do they reflect ethnic and regional preferences or vegetarian diets. EPA and FDA rely on USDA's Food Consumption Survey, which was completed in 1965-66. It is believed that some shifts have occurred in consumption patterns since then, and USDA is now conducting a new Food Consumption Survey.

\section{Evaluation of Decisionmaking Approach}

As discussed earlier, FDA regulates environmental contaminants in food under section 406 of the FD\&C Act. This section does not specifically address environmental contaminants, but authorizes FDA to regulate food for potentially toxic substances that are "added" and "unavoidable" in the production of food. When setting an action level or tolerance, FDA considers the impacts on the national food supply or, stated in another manner, the impacts on the availability of food to the American consumer. In quantifying this criterion, FDA estimates the amount of food that would be banned from commerce because of the action level or tolerance.

The final rule reducing РСB tolerances (8) illustrates FDA's interpretation of section 406 of the FD\&C Act for environmental contaminants in food. FDA clearly states that for PCBs "(i)t has had to decide, in effect, where the proper balance lies between providing an adequate degree of public health protection and avoiding excessive losses of food to American consumers."

FDA later goes on to state that:

(I)n establishing a tolerance for PCBs in fish, FDA must take into account the amount of fish a given tolerance would remove from commerce. Sect ion 406 of the Act, however, neither requires nor authorizes FDA to weigh secondary economic impacts when it considers the level at which a tolerance should he set. Consideration of such impacts would be inconsistent with the paramount concern of section 406, which is protection of the public health, and would complicate the decisionmaking process under section 406 in a way Congress did not intend. Obviously, consideration of the amount of food loss caused by a tolerance helps to ensure that the direct economic consequences of the tolerance (in this case, decreased sales and employment in the commercial fishing industry) will not be disproportionate to the increased degree of public health protection accomplished by the tolerance: but FDA considers secondary economic consequences, such as potential impact on the recreational fishing industry, totally beyond the scope of section 406 (8).

The decisionmaking process used by FDA is a form of cost-effectiveness analysis-a procedure to compare the change of health effects in biological terms with the change of the cost in dollars (a further analysis of this procedure appears in chapter VI). In the $\mathrm{PCB}$ decision, FDA compared change in human risk data for 5 parts per million (ppm), 2 ppm, and $1 \mathrm{ppm}$ levels in fish with the estimated amount of food that would be condemned in order to arrive at a 2-ppm tolerance level. In FDA's judgment, a 2-ppm tolerance was a proper balance between "providing an adequate degree of public health and avoiding excessive losses of food to American consumers." While other factors were considered by FDA in its decision, the estimated human risk data and loss of food are the two principal factors weighed in the decision.

The language of section 406 provides FDA with the flexibility to interpret the unavoidability requirement as it sees fit. FDA recognizes that its regulatory decision will have an economic impact, but FDA considers only a component of the total economic impact in its decision-i. e.. food condemned. FDA also realizes that the amount of food condemned will have an effect on employment and commercial sales associated with the contaminated food product. It must be recognized that even for such a widespread contaminant as PCBs, an action level for an environmental contaminant is likely to have a more severe impact on local employment and commercial sales than on the amount of food available to the American consumer. 
In addition, FDA's attempt to measure the impacts on availability of food by estimating cost of food condemned has several flaws. First, FDA estimates the amount of food expected to be condemned for one year from a proposed action level or tolerance. For substances such as PCBs in freshwater fish, estimates for the amount of food expected to be condemned should be for more than one year. This is because PCBs are ubiquitous in the environment and degrade very slowly. It is highly likely that freshwater fish will be contaminated with PCBs at levels above 2 ppm and consequently restricted from commerce for several years.

Second, estimating the cost of food banned by the tolerance does not necessarily reflect the impact on the availability of food. While this might occur for small amounts of food condemned-a herd of cattle or a few hundred gallons of milk-this would not be the case for incidents that condemn significant amounts of food. A more accurate estimate of the impact on the availability of food would be to consider the percentage or amount of a food product condemned out of the total amount of that particular product that is produced in this country, Then an estimate of the relative importance of the affected food product to the American diet would need to be made, FDA does not do this second step. This type of analysis would also attempt to estimate the impact such a tolerance would have on the supply and price of other foods available to and consumed by the American consumer.

While the latter analysis may be theoretically sound, given the time constraints for setting an action level for a newly discovered contaminant, it may not be practical for an initial regulatory decision. The more thorough analysis is more applicable to tolerancesetting.

\section{FEDERAL MONITORING PROGRAMS}

On the Federal level, the responsibility of monitoring foods for environmental contaminants falls mainly on two agencies, FDA and USDA. USDA limits its monitoring activities to meat and poultry products, while FDA analyzes samples of animal feeds, fruits, vegetables, grain, eggs, milk, processed dairy products, and seafood. Most monitoring activities of these two agencies could be classified as regulatory monitoring-analysis of food samples for known environmental contaminants and for some suspected environmental contaminants for enforcement purposes.

\section{Food and Drug Administration}

FDA collects approximately 8,000 food and feed samples a year and analyzes them for a variety of chemical residues, mainly pesticides. Domestic food commodities comprise about 70 percent of this total, imported commodities make up around 30 percent. In addition, approximately 1,300 seafood samples are collected for analysis annually. Most food and feed samples are collected at their point of origin or processing, and attempts are made to collect the seafoods as close to the point of origin as possible (9).

Some agricultural products are analyzed for the presence of trace metals-lead, zinc, and cadmium - as well as for synthetic organic chemicals. All fish samples collected for determination of chlorinated pesticides and PCBs are also analyzed for mercury. Some canned-tuna samples are analyzed for lead, cadmium, arsenic, selenium, and mercury. Because some containers have leadsoldered joints that may contaminate the food, an unspecified number of canned-food samples are analyzed to determine lead content (9).

FDA also determines the total dietary intake and exposure trends of some known and suspected environmental contaminants in its Total Diet Studies. The contaminants included in this program include some pesticides, PCBs, mercury, lead, cadmium, arsenic, selenium, and zinc, Other organics and metals are excluded (9). Because it involves 
not the analysis of individual raw food products but rather combinations of prepared cooked foods which approximate a total dietary intake, this program differs from the regulatory monitoring activities of FDA.

Monitoring for known environmental contaminants is carried out through the use of specified "accepted" methods for extraction, cleanup, and identification. The procedures, when applied to samples in which regulated synthetic organic chemicals such as pesticides are to be determined, will often indicate the presence of other compounds that are chemically similar to those under analysis. The FDA Compliance Program Guidance Manual (10) specifies that if, during the regulatory monitoring analyses, unidentified analytical responses appear (thus indicating an uncharacterized chemical) with "significant" intensity, data from the sample collection and analysis should be transmitted to the Bureau of Foods Laboratory in Washington, D. C., which presumably will identify the uncharacterized chemicals.

FDA may select a chemical for further study based on production volume, toxic byproducts, environmental stability, volubility, behavior, toxicity, uses, and methods of disposal. After an analytical method is developed, samples that have the highest probability of being contaminated with the particular (selected) compound are collected. These samples are often fish, since rivers, lakes, and estuaries receive chemicals not only from direct discharges from municipalities and industry but also from erosion and runoff. Further research and monitoring activities on a given chemical depend on the results of these initial analyses (9).

The present radionuclide-monitoring program is a joint undertaking by FDA, EPA, and the States, This program monitors: 1) foods grown near eight selected nuclear power facilities for tritium, gamma emitters, and strontium-90; 2) food samples from the total diet studies; 3) specified imported foods; and 4) milk, fruit, vegetables, and water collected near phosphate mines in Florida (11).
EPA monitors milk, air, water, and soil for radioactivity. The milk-monitoring program is a joint effort with State and local agencies, FDA advises and monitors the milk-sampling program, which is carried out by 63 State and local health agencies. Milk from each area is sampled once a month by the State and/or local inspectors and submitted for analysis to the EPA laboratory in Montgomery, Ala.

\section{U.S. Department of Agriculture}

USDA is responsible for evaluating the quality of meat and poultry products and providing the consumer with products that meet the criteria spelled out in the Meat and Poultry Inspection Acts. These criteria include monitoring for environmental contaminants. The majority of compounds evaluated are those that are approved for use in agriculture, either administered directly to food animals (such as growth promoters), or applied to agricultural crops to which food animals may eventually be exposed (such as pesticides).

Testing of meat and poultry products for residues by USDA falls into broad monitoring and surveillance categories. The monitoring activity, called the National Residue Monitoring Program, is designed to determine the frequency at which tolerance-exceeding amounts of monitored compounds are occurring in the national meat supply. In effect, the monitoring program is designed to evaluate how effectively users and/or manufacturers of the compounds are complying with the laws or use restrictions (12).

Under the monitoring program, animal tissues are collected from slaughterhouses under Federal inspection throughout the United States at a rate that will detect violations if they are occurring in at least 1 percent of the animal population [13). Based on statistical calculations, 300 samples per compound per species have to be collected annually to determine a 1-percent incidence with 95-percent assurance. In effect this means that the same sample of tissues may be analyzed for more than one compound. This level of testing re- 
quires sampling approximately 1 in 8,000 head of livestock and 1 in 700,000 poultry. These collections are stratified according to geographic areas, with more samples being collected from areas where meat or poultry are slaughtered.

USDA does not monitor all compounds for which tolerances have been established. There are no suitable methods to analyze some of them within existing regulatory monitoring laboratory capabilities. Available resources may also limit the number and variety of compounds tested, The selection of which compounds to monitor is based on factors such as frequency and patterns of use, toxicity, previous testing results, and public concern. Major groups of compounds in the monitoring program include synthetic organic chemicals (mainly chlorinated hydrocarbon insecticides), trace metals, antibiotics, sulfonamides, and certain hormones and drugs used for growth promotion and disease control. Organophosphate pesticides were monitored for several years but this monitoring was discontinued because residues of the parent compound were not found in animal tissue and suitable routine methods for detecting the metabolizes were not practical (12),

In 1978, about 150,000 tests were completed on approximately 20,000 domestic samples collected under the national residue monitoring program. An additional 2,000 samples were collected from imported products (12). Many of these samples are analyzed for potential contaminants other than trace metals and synthetic organics. In 1977, for instance, around 2,300 out of 22,000 samples were analyzed for chlorinated hydrocarbon pesticides and 1,300 for trace metals. The remainder were analyzed for antibiotics, hormones, and drugs (13). Data generated by this program are used not only for regulatory functions but also to help pinpoint problems, assist in trend analysis, and indicate areas that need more intensive sampling.

Surveillance samples are those collected to evaluate a problem. The area of sampling may be as small as a single farm or as large as a State or region, depending on the circumstances. Indications of problems come from many sources: the National Residue Monitoring Program, activities of USDA inspectors, information from State or other Federal officials, or from public news sources. Because these samples are biased (i.e., collected in response to a given need), they do not reflect the overall condition of the national meat supply. In most cases the samples are used to determine either the extent of a problem or to evaluate the acceptability of a herd or product. Any product found to be in violation may not be released into commerce until subsequent samples show that it is in compliance. These followup samples are considered surveillance samples,

Analyses of samples collected in USDA programs are performed in a manner similar to those in the FDA program-prescribed analytical methods are used. USDA, like FDA, may try to identify an "uncharacterized" substance when "unknown" peaks appear in the analysis of a sample. Depending on the identity of the compound, further investigation and sampling may be carried out,

\section{Environmental Protection Agency}

EPA has no mandate to regularly analyze food commodities for chemical contamination. Some of its programs designed to determine the ecological impact of pollutants may include certain types of foods for analysis. This is particularly true in the case of seafood. Samples from aquatic food chains are often selected for analysis to ascertain whether a pollutant is concentrated as it moves up the food chain.

In addition, the mobilization, degradation, and transfer of pollutants is often studied by EPA. For example, under the auspices of EPA's Chesapeake Bay program, trace metals and synthetic organic chemicals are being studied in water, living organisms, and sediments of the Chesapeake Bay by various State agencies and academic institutions. The results of this and similar studies are not designed to protect the public from consuming contaminated food and are seldom used as 
such. Rather, the results are used in protecting the environment from chemical insults and helping EPA better regulate the introduction of toxic substances into the environment.

Some data transfer between EPA and other Federal agencies exists. For example, in the EPA-funded kepone studies in the Chesapeake Bay region, kepone concentrations in edible fish, shellfish, and crabs are obtained which assist the Commonwealth of Virginia and FDA in their efforts to keep contaminated sea food off the market. This is true even though the study is not designed specifically for this purpose.

Another program administered by the EPA is the Mussel Watch (14). It is designed to analyze shellfish collected from strategic locations in the marine coastal zone of the United States for selected organic chemicals, trace metals, and radionuclides. This program was started in 1976 and now involves the collection of oysters and/or mussels from 29 stations on the west coast, 34 stations on the east coast, and 26 stations on the gulf coast, Collections from these stations make up a total of 107 samples,

The trace metals for which samples are analyzed in this program include lead, cadmium, silver, zinc, copper, and nickel. The radionuclides measured in the samples include plutonium-238 and -239, americum-241, cesium-137, curium-242 and -244, and lead-210. The synthetic organic chemicals included in the analysis are the halogenated hydrocarbons $p, p^{\prime}-D D E$ and p,p'-DDD-two of the principal breakdown products of the pesticide DDT-and PCBs. Samples are analyzed for these synthetic organic chemicals as well as petroleum hydrocarbons, whose presence may indicate oil pollution. Recently, a number of uncharacterized substances have been found by Mussel Watch scientists. They are now designing the program to encompass systems to track and identify them (15).

\section{Critique of Federal Monitoring Programs}

A chemical substance found in food becomes the subject of Federal regulatory moni- toring if it has caused a problem at some time in the past. In other words, a compound may enter the food supply and be undetected for years but categorized as a known contaminant when discovered. An example would be kepone in seafood of the James River in Virginia (16). This compound is an insecticide that had been entering the aquatic food chain for at least 7 years before discovery. Its presence in seafood was determined after workers in the facility that manufactured it became ill from industrial exposure to this toxicant (17)0

As soon as the sick workers were discovered, samples of fish and oysters from the adjacent river were analyzed and shown to contain kepone. Within a few months, action levels were established for kepone in seafood. Because existing monitoring programs analyze for known regulated environmental contaminants, the compound was not discovered because it was not sought. It is unlikely that the presence of kepone would be known and regulated today had the workers not become ill. In this case, kepone was placed in the known-environmental-contaminants category as a result of the illness of production workers, not chemical monitoring.

Another example of a toxic compound entering the food supply and going undetected is the fire-retardant polybrominated biphenyls (PBBs), PBBs entered the food supply in Michigan in the late spring or early summer of $1973(18,19)$, Bags of a fire retardant containing these compounds were shipped to farmers' cooperative units in Michigan in place of the intended livestock feed supplement, The fire retardant was unknowingly mixed with cattle feed and fed to herds throughout the southern part of the State. Even though farmers soon noted the symptoms of poisoning in the cattle and reported the problem to State and Federal officials, it was almost a year before the causative agents, PBBs, were identified. During this year dairy products and beef contaminated with these compounds were consumed by the citizens of Michigan. Soon after the compound was identified in food products, action levels were established to protect the consumer. In this case the trig- 
ger for designation of PBBs as known environmental contaminants was sick cattle, not chemical monitoring.

From these two case studies it appears that monitoring programs designed mainly to check for known environmental contaminants are insufficient to detect toxicants in food for which there have been no action or tolerance levels established.

\section{CHAPTER III REFERENCES}

1. Federal Food, Drug and Cosmetic Act, 21 U.S.C. 321 et seq.

2. Poultry and Poultry Products Inspection Act, 21 U.S.C. 451 et seq.

3. Federal Meat Inspection Act, 21 U.S.C. 601 et seq.

4. Merrill, R.A. FDA Regulation of Food Safety, unpublished manuscript, 1978.

5. Kennedy, D., Commissioner, Food and Drug Administration, to J. B. Cordaro, Jan. 22, 1979,

6. 21 CFR 109.

7. Arnold, F. T. Kepone Action Levels-Review, Analysis and Recommendations, Office of Special Pesticide Reviews, Environmental Protection Agency, Mar. 9, 1977.

8. "Polychlorinated Biphenyls (PCBs), Reduction of Tolerances," Federal Register 44(127): 38330, 1979.

9. Food and Drug Administration. Report in Response to the Office of Technology Assessment letter of June 5, 1978- "Request for Information on Federal Capabilities for Environmental Monitoring and Toxicity Testing, " 1978.

10. Food and Drug Administration. Compliance Program Guidance Manual, Compliance Program 7305.007 -' Pesticide and Metals in Fish Programs" (FY '78), Oct. 1, 1977.

11. Food and Drug Administration, Compliance Program Guidance Manual, Compliance Program 7305.006-'" Radionuclides in Foods" (FY “78), Oct. 1, 1977.
12. Clark, G. U.S. Department of Agriculture, 1979.

13. U.S. Department of Agriculture. The National Residue Program, USDA-Food Safety and Quality Service, May 1978.

14. Goldberg, E, D., V. T. Barrington, J. W. Harvey, G. H. Martin, J. H. Parker, P. L. Risebrough, R. W. Robertson, W. Schneider, E. S. and E. Gamble. "The Mussel Watch," Environmental Conservation, vol. 2, Summer 1978 ,

15. Risebrough, R. W. Bodega Marine Laboratory, University of California, Bodega Bay, Calif., personal communication, 1978.

16. Huggett, R. J., M. M. Nichols ${ }_{s}$ and M. E. Binder. "Kepone Contamination of the James River Estuary, " Proceedings of Symposium on Contaminants and Sediments: Fate and Transport, R. A. Baker (cd.), Honolulu, Hawaii, April 1979 (in press),

17. Food and Drug Administration. Compliance Program Evaluation (FY '77), Kepone and Mirex Contamination (7320.79A).

18. McNally, J. M, "Polybrominated Biphenvls: Environ-mental Conta-mination of Food," special paper for OTA, Library of Congress, Congressional Research Service, 1978.

19 Michigan Department of Agriculture, Information and Education Division. "A Brief Chronology of the PBB Incident" (no date). 
Chapter IV

\section{State Laws, Regulations, and Programs}




\title{
State Laws, Regulations, and Programs
}

\begin{abstract}
STATE LAWS
State food and drug laws, and the organizations that administer them, vary widely. Basic State food and drug statutes are based on the Federal food laws; however, not all States have adopted the model uniform State food, drug, and cosmetic bill of the Association of Food and Drug Officials. As shown in figure 3, 42 States have adopted the model statute, which is almost identical to the 1938 Federal Food Drug and Cosmetic Act. Consequently, these 42 States have the same legislative authority for regulating food contaminants within their borders as the Federal Government does for food in interstate commerce. The 1906 Act, still retained by eight States, does not contain the tolerance-setting provisions of the 1938 Act under which environmental contaminants are regulated (l).
\end{abstract}

Authority for regulating environmental contaminants in food rests with two or more agencies in most States. Usually the Department of Agriculture and the Department of Health share responsibilities for food regulation. In some States a variety of other agencies and bodies are also involved in regulatory or research activities: departments dealing with commerce, fish and game, consumer protection, environmental improvement, public administration, conservation, along with university divisions, independent laboratory agencies, and various independent boards and commissions (2).

The Food and Drug Administration (FDA) believes that this variability in laws and regulatory organizations makes it more difficult for the States to accomplish their goals:

The variability of organizational structure complicates the problems of many of the individual State agencies in accomplishing their program goals because of overlapping responsibilities and the lack of a clear delineation of responsibilities. For example, it is not uncommon to find authority granted to two agencies for some divided program segments of a single program category (e.g.,
Figure 3.- State Food Laws

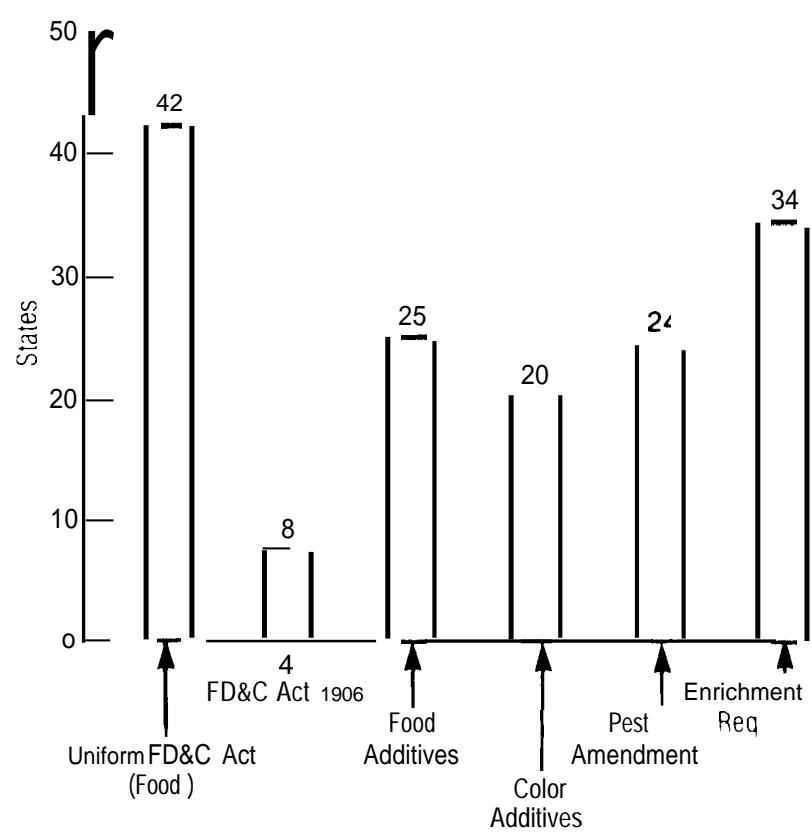

SOURCE Food and Drug Administration State Programs and Services in Food and Drug Control, 1978 
milk, shellfish). Frequently, two or more independent agencies of relatively equal rank are charged with enforcement of portions of the same general food and drug law. In still other States, there is no central State control over the food and drug program. In these in- stances, the State agency has an unclear role as an advisor or consultant to the local government. However, the local agency may not be legally bound to follow the advice and/or direction that may be suggested by the State agency (1).

\section{STATE Monitoring PROGRAMS}

Non-Federal monitoring is limited almost exclusively to programs originated and carried out by individual States. In some cases there is close coordination between State and Federal activities. Federal agencies may decrease their monitoring in a given area if State monitoring is considered sufficient. For example, FDA does not monitor seafood for kepone (3) even though the concentrations in finfish, crabs, or shellfish remain essentially unchanged since its discovery in 1975 (4). FDA feels that the ongoing monitoring programs in Virginia are sufficient to protect the consumer.

Analysis of the OTA State survey reveals that State food-monitoring laboratories are equipped to analyze for those substances that are regulated in foods through action levels or tolerances. For instance, most had instrumentation (atomic absorption spectrophotometers and gas chromatography) to analyze for mercury and chlorinated hydrocarbon pesticides.

FDA published a study entitled "State Programs and Services in Food and Drug Control" in September 1978 (1). The publication provides a compilation of States' analytical capabilities during the years 1975 and 1976, listing numbers and educational levels of chemical analysts, types of analytical equipment, and expenditures for food inspection.

In 1974 and 1975, the States spent annually about $\$ 64.9$ million for food inspection and analytical activities. This amounted to 72 percent of their total inspection and analytical expenditures (1). Table 8 shows program areas and expenditures for States. These
Table 8.-Program Areas and Expenditures for States

\begin{tabular}{|c|c|c|}
\hline \multirow[b]{2}{*}{ Program } & \multicolumn{2}{|c|}{ Expenditures } \\
\hline & $\begin{array}{l}\text { Millions } \\
\text { dollars }\end{array}$ & $\begin{array}{l}\text { of Percent of total } \\
\text { (food and drug) }\end{array}$ \\
\hline $\begin{array}{l}\text { Food } \ldots \ldots \ldots \\
\text { Drugs, devices, cosmetics } \ldots \\
\text { Feed } \ldots \ldots \ldots \\
\text { Weights and measures (food) } \\
\text { Pesticides } \ldots, \ldots, \ldots\end{array}$ & $\begin{array}{r}\$ 64.9 \\
9.7 \\
6.4 \\
5.5 \\
3.3\end{array}$ & $\begin{array}{r}72.3 \\
11.0 \\
7.0 \\
6.0 \\
3.7\end{array}$ \\
\hline Total & $\$ 89.8$ & 100.0 \\
\hline
\end{tabular}

figures do not include the estimated $\$ 75$ million expended by local governments.

The educational levels of the chief chemist, supervisory chemist, and chemist and laboratory technicians are presented in table 9 (1). The salary ranges for the chemical personnel are shown in table 10 (l). Only 1 percent of the chemists working in State food and drug programs earned more than $\$ 20,000$ per year in 1975 and 1976. Approximately 64 percent of them had annual salary ranges of $\$ 8,000$ to $\$ 15,000$.

The available analytical equipment and physical facilities are listed in table 11 (1). These data confirm the OTA survey findings, since gas chromatography and atomic absorption spectrophotometers rank first and third, respectively, in numbers. A breakdown of food commodities analyzed, samples collected, and analyses performed is given in table 12 (1). The FDA document urges caution in interpreting these data because ". . . some States do not maintain comprehensive analytical records on food analyses especially if food is not the major laboratory workload." 
Table 9.-Number of Employees by Category and Education Level in Various State Food and Drug Programs

\begin{tabular}{|c|c|c|c|c|c|}
\hline Personnel & Number of employees & Advanced degree & College degree & Some college & No college \\
\hline Chief chemist. . & 109 & 68 & '4 0 & 1 & 0 \\
\hline oervisory chemist & 232 & 62 & 167 & 3 & 0 \\
\hline Chemist. . . . . . . . . . & 633 & 66 & 561 & 6 & 0 \\
\hline oratory technicians & 833 & 0 & 127 & 375 & 332 \\
\hline
\end{tabular}

SOURCE Food-and-Drug Administration State Programs and Servicesin food and Drug Control 1978

Table 10.-Salary Ranges of Various Chemical Personnel in State Food and Drug Programs

\begin{tabular}{|c|c|c|c|}
\hline Salary-ranges & Number of chief chemists & $\mathrm{Nu} \mathrm{m}$ ber of $\mathrm{s} u$ pervisory chemists & Number of chemists \\
\hline$\$ 6,000-9,000 \ldots \ldots \ldots \ldots$ & 0 & 3 & 0 \\
\hline$\$ 7,000-10,000 \ldots \ldots \ldots \ldots$ & 0 & 0 & 26 \\
\hline$\$ 8,000-12,000 \ldots$ & 0 & 0 & 70 \\
\hline$\$ 8.000-14,000 \ldots \ldots \ldots$ & 2 & 17 & 153 \\
\hline$\$ 10,000-15,000$ & 2 & 143 & 139 \\
\hline$\$ 12,000-18000 \ldots \ldots \ldots \ldots \ldots$ & 17 & 53 & 5 \\
\hline$\$ 16,000-20,000 \ldots \ldots \ldots$ & 71 & 7 & 0 \\
\hline over $\$ 20,000 \ldots \ldots \ldots$ & 8 & 0 & 0 \\
\hline
\end{tabular}

SOURCE Food and Drug Administration State Programs and Services in Food and Drug Control 1978

Table 11 .-Physical Facilities and Key Equipment in $114^{\text {a }}$ State Food and Drug Laboratories

Key equipment items
Spectrophotometers . . . . . . . . . . . . . .

This probably accounts for the extensive analytical activity reported in the "other food" category, rather than in the categorical program areas"' (l). Even so, it is evident that milk and milk products are the most commonly sampled and analyzed food commodities.

The large number of samples and analyses indicate that the States perform extensive monitoring for regulated contaminants in food, But the low salary ranges, the lack of sophisticated analytical equipment such as mass spectrometers, the time spent per analysis, and the sample-type distribution indicate that State monitoring programs are as inadequate as Federal programs in detecting environmental contaminants in food for which no action or tolerance levels have been established, 
Table 12.-Total Number of Samples, Sample Determinations, and Man-Hours by Food Commodity Categories With Number and Percentage of States Reporting Analytical Activity

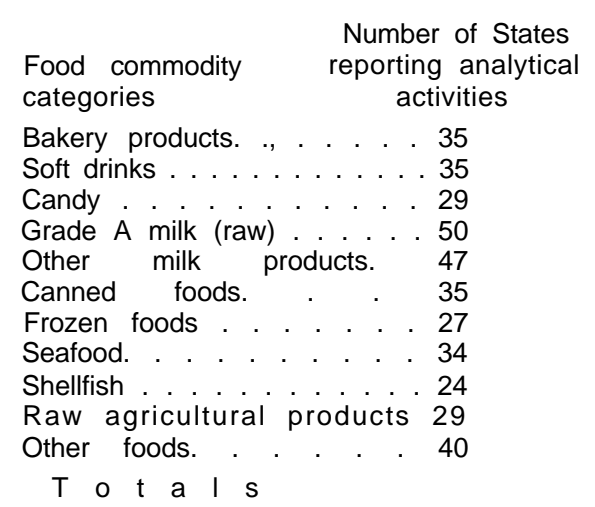

$\begin{array}{cc}\text { Percent of States } & \begin{array}{c}\text { Number of samples } \\ \text { (thousands) }\end{array} \\ 70 & 11.5 \\ 70 & 18.7 \\ 58 & 7.0 \\ 100 & 342.0 \\ 94 & 200,7 \\ 70 & 21.3 \\ 54 & 7.3 \\ 68 & 6.6 \\ 48 & 37.6 \\ 58 & 40,1 \\ 80 & 103,0 \\ & 7958\end{array}$

$\begin{array}{cc}\begin{array}{c}\text { Number of } \\ \text { determinations } \\ \text { (thousands) }\end{array} & \begin{array}{c}\text { Number of man- } \\ \text { hours }\end{array} \\ \text { (thousands) }\end{array}$

SOURCE Food and Drug Administration S/ate Programs and Servicesin Food and Drug Control 1978

\section{FEDERAL/STATE LIAISON}

Many environmental contamination incidents are initially State problems. Theoretically, the Federal Government does not become involved until a contamination incident is determined to be an interstate problem. Given the complexity of this country's foodmarketing system, most food produced or processed within a particular State is distributed for consumption in other States. Thus, most environmental contamination incidents are likely to become interstate concerns. Figure 4 reveals the extent of food contamination that can occur from a single source of contamination, in this instance polychlorinated biphenyls (PCBs) contaminated animal feed from a meatpacking plant in Billings, Mont. (5). This widespread contamination of food occurred during an estimated time period of 2 to 5 months.

The States and the Federal Government establish liaison when contamination crosses State lines. Liaison is also established at the request of States. States often require Federal assistance in investigating a contamination incident. The objective is to generate scientific information on the nature and extent of the contamination. This information would include the toxicological and chemical properties of the contaminating substance, the amount and type of food contaminated, and the concentration of the substances in food.
Such information is used by State and Federal authorities to: 1) determine the appropriate Government response for protecting the public health and 2) inform the general public about the incident and explain the Government response. To contain an incident, scientific information needs to be accurate and immediately available. This is as true for an episode involving a substance that has previously contaminated food (1979 PCB contamination of animal feed in Billings, Mont. ) as for a substance which has not (1973 polybrominated biphenyl (PBB) contamination of animal feed in Michigan).

The generation and dissemination of scientific information on an incident is hindered by the number of State and Federal agencies involved. As already noted, three Federal agencies (the Environmental Protection Agency (EPA), FDA, and the U.S. Department of Agriculture (USDA)), each with different responsibilities, can be involved along with various State agencies. The PBB incident in Michigan and the PCB incident in Montana reflect this particular problem.

\section{Generating Information}

Before the needed scientific information for developing regulations is generated, the 


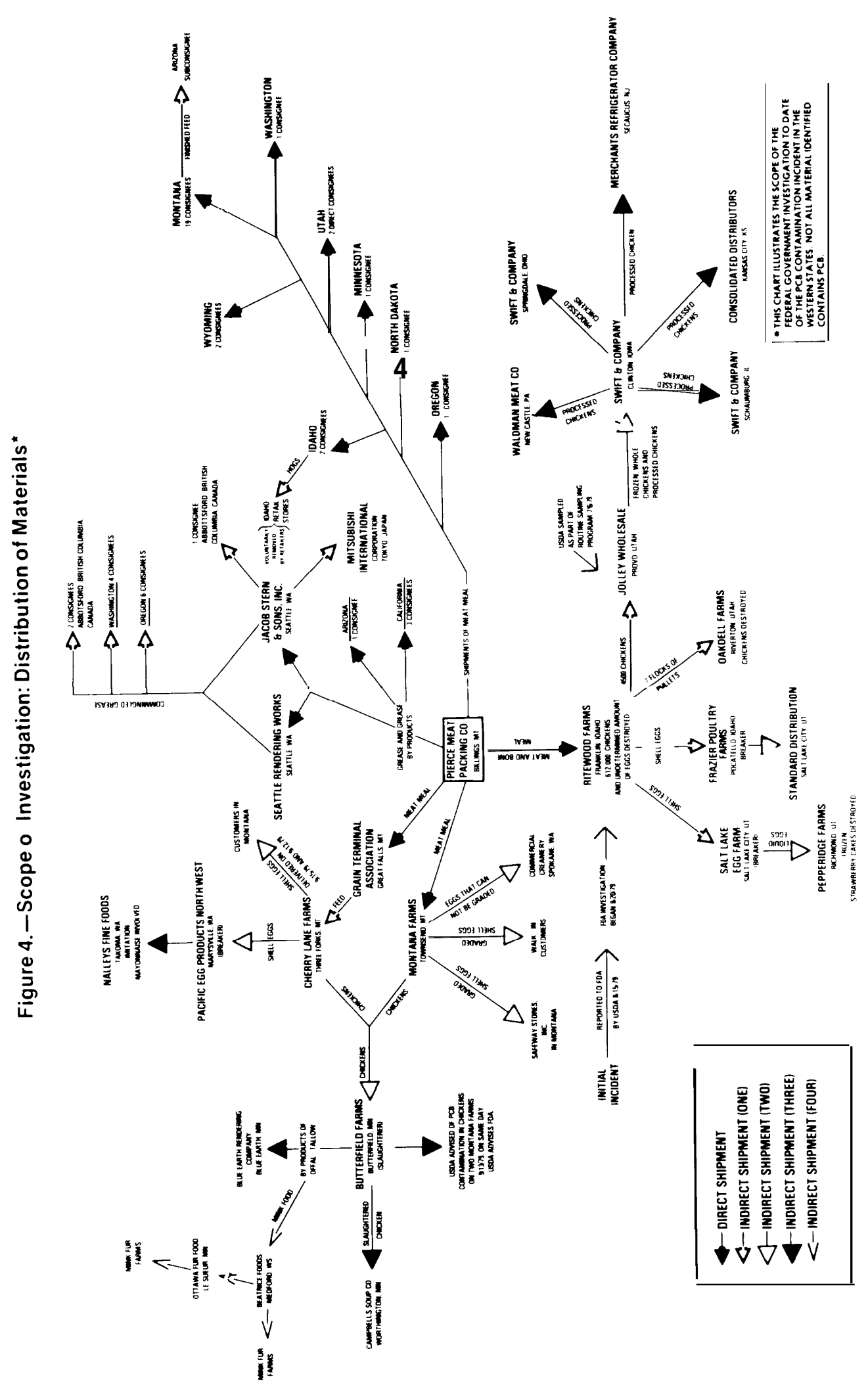


contaminating substance in food must be identified. This identification process may require lengthy investigative work which could be hindered by a multiplicity of involved Federal and State agencies. The PBB incident provides an example of the extensive investigations sometimes necessary.

The Michigan Department of Agriculture (MDA) initially analyzed blood and feed samples from a herd owned by Mr. Frederic Halbert (at his urging). These samples proved to be negative because MDA was not analyzing for PBB. Additional samples were analyzed by the USDA's National Animal Disease Center in Ames, Iowa. While the Disease Center detected an unusually high peak of an unidentified substance, it did not determine its identity. The substance was eventually identified by Dr. George Fries of USDA's Agriculture Research Center at Beltsville, Md., who had previous experience in chemical analysis of PBB (6). The discovery came nearly 8 months after adverse symptoms occurred in the herd at the Halbert farm.

The laboratories and agencies involved in identifying PBB are part of the agriculture system in the United States. They were the obvious institutions to which Mr. Halbert would go for assistance. If he had initially contacted the Michigan Department of Public Health (MDPH), it is likely that different Federal laboratories and agencies would have analyzed the samples. There was and still is no systematic procedure at the Federal level for assisting States in identifying a potential food contamination problem like PBB. Were it not for the perseverance of Mr. Halbert and the experience of Dr. Fries, the identity of PBB might have taken longer and the people of Michigan would have been exposed to PBB-contaminated food even longer.

Once a contaminant has been identified in food, the necessary scientific information can be generated for either regulating or controlling the contamination. In the PBB incident, FDA helped to develop and evaluate this information and worked with MDA in controlling the incident. FDA's involvement was based on the fact that it has the Federal authority for regulating contaminants present in food. Examples of actions taken by State and Federal authorities in such instances include removing food from the market, setting action levels or tolerances for the contaminant in food, and disposing of the contaminated food. Because little scientific information on PBBs was available, it took time to generate the information and establish final permissible levels (although contaminated herds and food were identified as quickly as possible and removed from the market). The PBB incident involved FDA, USDA, MDA, and MDPH. Even more State and Federal agencies were involved in the PCB contamination episode in the Western United States.

The PCB contamination began in Montana with animal feed and quickly spread to 16 other States. Idaho was particularly affected by the contamination of poultry and eggs. This incident involved all three relevant Federal agencies-FDA, EPA, and USDA-as well as the Idaho Departments of Agriculture and Health and Welfare, and district health departments. USDA made the initial analysis of poultry samples which proved positive for PCB; FDA was involved with the removal of PCB-contaminated food from the market; and EPA with the proper disposal of the contaminated food. At least 5 days elapsed from the time USDA was confident it had a PCB contamination incident to the time it notified FDA of its findings. It took FDA an additional 5 days to begin its investigation of the contamination incident (7). Such delays would be unlikely if only one Federal agency were involved or communications between the two agencies were better.

PCB is a substance whose chemical and toxicological properties are fairly well understood. It has contaminated food in the past. Nevertheless, there was confusion among the State agencies in Idaho as to the proper response to the contamination. The confusion resulted from two conditions. First, some of the State officials involved were not familiar with the chemical and toxicological properties of PCB. PCB was a new food con- 
taminant in Idaho, and the appropriate officials had little experience with this type of problem. Second, the involvement of three different Federal agencies obstructed efficient communication between the State agencies and the Federal Government.

While the Federal agencies had the most expertise on PCBs, the sharing of that experience was hindered by the fact that it was available in and distributed by three sources instead of one. Consequently, State agencies had to go to different Federal sources, depending on what information they needed. In addition, the Federal agencies did not always communicate with the various State agencies. EPA, for example, took air and water samples in the area surrounding Ritewood Farms to determine whether PCBs that contaminated Ritewood's eggs and poultry came from either of these two sources. This was before the PCB-contaminated animal feed was identified. EPA, however. did not report their negative findings to the State. In another instance, USDA initially would report its results only to the Idaho Department of Agriculture, the State agency with which USDA has had a long-standing association. The Idaho Depart- ment of Health and Welfare, which is concerned with protecting the public health, at first was not informed by either USDA or the State Department of Agriculture of the PCB contamination. Communication broke down at two levels, between the State and Federal Governments and within the State government (8). The fact that there are several different Federal and State agencies involved with different aspects of controlling and regulating a contamination incident further complicates an already complicated problem.

The major environmental contamination incidents that occurred in Idaho and Michigan continue to be major issues of concern among the residents of these States--a result of their fears over a potential health threat that cannot be seen, smelled. or tasted. In Michigan, for instance, the PBB episode remains a live and controversial political issue. Consequently, it becomes imperative that the information generated by the State and/or Federal Government on an incident is accurate and appropriately applied. This objective is hindered by the variety of State and Federal agencies that become involved.

\section{CHAPTER IV REFERENCES}

1. U.S. Department of Health. Education, and Welfare, Food and Drug Administration. State Programs and Services in Food and Drug Control, 1978.

2. OTA Survey of 31 States, 1978.

3. Food and Drug Administration. Compliance Program Evaluation (FY '77), Kepone and Mirex Contamination ( 7320. 79A).

4. Huggett, R. J., M. M. Nichols, and M. E. Binder. "Kepone Contamination of the James River Estuary, " Proceedings of Symposium on Contaminants and Sediments: Fete and Transport. R. A. Baker (cd.), Honolulu, Hawaii. April 1979 (in press).

5. Gardner, Sherwin, Acting Commissioner of Food and Drug Administration. Testimony be- fore Subcommittee on Oversight and Investigation, House Committee on Interstate and Foreign Commerce, Sept. 28, 1979.

6. Michigan Department of Agriculture, Information and Education Division. "A Brief Chronology oft he PBB Incident ," (no date).

7. Foreman, Carol Tucker, Assistant Secretary for Food and Consumer Services, U.S. Department of Agriculture. Testimony before the Subcommittee on Oversight and Investigation, House Committee on Interstate and Foreign Commerce, Sept. 28, 1979.

8. Gallagher, Ed, State Health Officer, Idaho Department of Health and Welfare, personal communication, Oct. 10, 1979. 
Chapter V

\section{Methods for Assessing \\ Health Risks}




\section{Methods for Assessing Health Risks}

The availability and quality of data on the toxicity of contaminants in large part determine the Food and Drug Administration's (FDA) ability to protect public health. Chapter III reviewed the strengths and weaknesses of FDA's procedures for setting action levels and tolerances. This chapter addresses four issues related to the methods used for assessing health risks:

- What is the role of human epidemiology in the setting of action levels and tolerances?

- What are the roles of animal and other toxicological tests?

- Are current testing procedures adequate for the assessment of interactions of toxic substances?

- Are methods for quantitative risk assessment sufficiently developed for application to environmental contaminants in food?

\section{POSSIBLE TOXIC EFFECTS}

The possible toxic effects of an environmental contaminant depend on its chemical nature, its concentration in food, and the type of toxic action involved. If the substance is highly toxic and/or is consumed in large quantity, acute toxic effects may occur and the onset of the symptoms would be rapid and severe. If a small amount of a highly toxic substance is consumed, or if the substance is of low toxicity, the health effects may not be seen for many months or years (or may not be observed at all). Potential effects of toxic environmental contaminants in food include systemic toxicity, mutations, cancer, birth defects, and reproductive disorders.

Systemic toxicity involves changes in the structure and function of organs and organ systems: weight change, structural alterations, and changes in organ system or whole animal function. Functional effects may include changes in the lungs, liver, and kidneys, in cardiovascular function, in brain and nervous system activity and behavior, and in resistance to disease (1). Systemic toxicity is studied in whole animal tests.

Some environmental contaminants have been shown in experiments to cause point mu- tations (which generally affect a single gene) or more extensive effects such as gross changes in chromosome structure or changes in chromosome number. Such genetic effects in humans often cannot be immediately detected. Indeed, damage to the human gene pool may not become apparent for many generations if the deleterious effect is due to a recessive gene (2). However, a chemical's mutagenic potential can be evaluated indirectly from various biological tests involving microorganisms, mammalian cell cultures, insects, and whole mammals (1).

Some environmental contaminants in food may cause cancer. Direct cause-and-effect associations between exposure to a specific chemical and human cancer are difficult to establish because of the complex nature of cancer and the vast number of potential carcinogens to which humans are exposed. In some cases, exposure to one toxic agent may trigger a sequence of events leading to cancer. In others, carcinogenesis may depend on interactions of several factors, combinations of noxious agents, co-factors, and natural or acquired metabolic peculiarities (2). The cancer-causing potential of a sub- 
stance is evaluated through animal tests and a variety of short-term tests employing bacteria, insects, or cell cultures (1).

Environmental contaminants may produce birth defects other than inherited mutations. These are called "teratogenic effects. " Malformations may include gross, histological, molecular, and sometimes behavioral anomalies. Human sensitivity to a teratogenic agent during pregnancy is determined by: 1) the time at which the insult is received during gestation, 2) the dose of the compound, 3) transfer of the teratogen to the developing fetus, 4) uterine factors, and 5) dietary factors. The teratogen may work its effect by producing mutations or chromosomal aberrations, interfering with cell division, altering nucleic acid synthesis, inhibiting specific enzymes, or altering membrane characteristics. A seemingly small change may have far-reaching effects, since the fetus is undergoing rapid biochemical, structural, and physiological growth and change (1).
Environmental contaminants can have other effects on the reproductive process in both males and females. Toxic effects may include reduced or altered sperm formation, inhibition of ovulation, increased spontaneous abortion, fetal resorption, and increased numbers of stillborn infants, Teratogenic and reproductive effects are evaluated through animal tests (1).

Given the range of possible adverse health impacts, it is clear that newly discovered environmental contaminants must be subjected to the best available toxicological testing techniques so that any harmful effects can be uncovered. Furthermore, regulators must have information on the possible toxic effects of ingesting small amounts of a substance in food over an extended period of time, perhaps over a lifetime. It would also be desirable to know what effects other toxic substances already present in our air, water, and food may have on the metabolism and toxicity of a new contaminant,

\section{HUMAN EPIDEMIOLOGY}

The science of epidemiology seeks to determine the distribution and causes of diseases and injury in humans, It focuses on groups rather than individuals (3).

There are several types of epidemiological studies. Each provides different levels of information on environmentally related disease or injury. The cohort-study method is the best way to develop information on potential toxic effects in a population exposed to an environmental contaminant in food. In a cohort study, individuals are classified into groups according to the levels of exposure, including a control group with no previous exposure to the suspect substance. The groups are then followed over a period of time and studied for differences in disease incidence (3). Such epidemiological studies have an advantage over animal tests. There are some agents known to cause disease in humans that do not produce similar adverse effects in animals (benzene, for example) (4). Human epidemiological data can also be used to directly estimate human risk, thus eliminating the need for extrapolating from animal experiments.

Epidemiological evidence has sometimes weighed heavily in the setting of tolerances or action levels. This was the case in FDA's decisions on mercury and polychlorinated biphenyls (PCBs). However, if epidemiological data are lacking when an environmental contaminant is discovered in food, the usefulness of further epidemiological studies is restricted by inherent limitations of the science.

Beyond the obvious ethical considerations, such studies are of little use when a rapid regulatory decision must be made. At the low levels that environmental contaminants usually occur in food, pathological effects may not occur for many years, Most cancers, for example, have a latency period of at least 5 to 10 years, As much as 40 years may elapse between the onset of exposure and the development of the disease, Thus epidemiological 
methods can only be used retrospectively to evaluate the health status of individuals who have been through this 5- to 40-year period. The findings are compared to the health histories of "control" individuals who presumably have not experienced comparable exposures.

Such retrospective studies are vulnerable to scientific criticism: a) it is very hard to find an adequate control group (unlike animals whose entire lives can be in a "controlled" environment) because of the variability in human susceptibility and personal behavior patterns, b) it is impossible to define potential synergisms and/or inhibitions by other substances to which the target group may have been exposed, and c) it is difficult to quantify previous levels of exposure (5).

Retrospective studies suffer other handicaps, They are extremely expensive, difficult, and time-consuming to carry out, A given type of cancer, for example, may occur in the "normal" population at a rate of 1 case for each 100,000 people. An exposure to a toxic substance that increases by a factor of 100 the likelihood of that cancer occurring would raise the incidence to 1 case for each 1,000 people. To ascertain in a statistically satisfactory and compelling way that such an increase had occurred, one would have to do comprehensive medical studies on at least 3,000 people. Assuming an average cost of $\$ 200$ for each person studied (which is a low estimate considering the costs of physicians" time, laboratory analysis, and the technological assessments necessary to show a person free of a given tumor or disease), the expense for such a study would be $\$ 600,000$. It would be necessary under most circumstances to find more than one new case of a disease to convince a nonstatistician and even most statisticians. This would double or triple the costs (5). Furthermore, demonstrating that the incidence had increased would still leave the vexing problem of correlating the increase with the exposure to the specific suspect substance.

Such limitations make cohort epidemiology studies inappropriate for determining the toxic potential of a given contaminant. Moreover, in a regulatory scheme designed to prevent serious illness from developing in humans, the time required to generate epidemiological data would preclude its use in initial regulatory decisions. The prospects for developing a human epidemiological method that would meet such regulatory demands are presently hard to imagine.

One area, however, where human epidemiological studies have and will continue to be useful is in the confirmation of suspected chronic-effect data when a population that has inadvertently been exposed to a substance or a hazard (such as radiation) over a long period of time can be identified. In this instance, carefully designed studies can be the "clincher"" which finally provides thorough documentation of the suspected toxicity. Such studies can confirm or refute the adequacy of regulatory actions (in retrospect) (5), Again, however, these kinds of studies are extremely expensive and consume limited resources which might be better spent supporting other types of evaluations using other techniques.

People who fail to understand these limitations criticize reliance on animal tests when epidemiological information is negative or lacking. This fundamental misunderstanding may delay health-related regulatory action and dilute its effectiveness in preventing illness. 


\section{ANIMAL TESTS AND OTHER METHODS FOR TOXICOLOGICAL TESTING}

Toxicologists have developed a number of techniques to assess the toxicity of a compound. Some tests can be conducted in 90 days or less. These include simple tests such as 2-hour "range finding" to determine the dose of a substance that is lethal to 50 percent of the animals (LD50). More complex tests include 90-day continuous exposure or paired feeding studies, and short-term tests for mutagenesis and potential carcinogenesis. Tests requiring more than 90 days, such as lifetime exposure studies, are generally considered long-term. In addition to the time necessary for exposure and data-gathering, analysis of the results may take up to an additional year, depending on the complexity of the experiment, the number of animals used, and the amount of data collected (1).

Testing methods can be categorized by "endpoint" as well as duration. Some experiments are based on expected results such as functional changes, birth defects, or cancer, By the use of an appropriate experimental design, several such endpoints can be assessed in the same experimental period (l). Appendix $C$ describes the range of toxicological tests available for assessing the health risks associated with consumption of contaminated food. Each section describes the tests for assessing a given endpoint, and includes a discussion of available long- and short-term tests,

Animal tests provide valuable information for the setting of action levels and tolerances. The animals serve as proxies for humans in cases where data are lacking on the human health effects of a contaminant and experiments involving humans would be unethical, Because of shorter animal lifespans, the effects on several generations can be studied. Furthermore, animals can be raised in controlled environments, thus eliminating many of the factors that complicate human epidemiological studies, In many cases, animal experiments are the only means by which needed information can be obtained. Using a carefully selected battery of 90-day tests, considerable data can be generated on a contaminant's potential toxic effects including its potential for causing mutations and cancer.

Despite widespread scientific agreement on the usefulness of animal tests, several controversies exist. These include the appropriateness of the carcinogen bioassay techniques, the potential of short-term tests for evaluating mutagenesis and carcinogenesis, the lack of emphasis on testing for potential toxic interactions, and the usefulness of methods for extrapolating from high-dose animal test results to low doses in humans.

\section{The Carcinogen Bioassay}

The carcinogen bioassay (6) is a chronic toxicity study requiring 2 years' exposure to the test substance and usually a third year for evaluation of the results. The carcinogen bioassay is used to determine the cancercausing potential of a compound in males and females of two mammalian species, usually the rat and mouse. The test animals are exposed to the test substance throughout their entire lifespan.

Different groups are exposed to different levels of the substance up to the maximum tolerated dose (MTD). The MTD is the highest dose given during a chronic study that predictively will not alter the animals' normal longevity from effects other than cancer. In practice, the MTD is considered to be the highest dose that causes no more than a 10percent loss in weight compared to control animals. Throughout the study, animals are examined weekly for signs of toxicity. Animals may be killed at prearranged times and their tissues examined for signs of cancer. At the completion of the study, all remaining animals are killed and detailed examinations are made of their tissues.

Animal tests for carcinogenicity have been questioned because the results are generalized to humans while the animals are fed 
much larger doses of the suspected substance than would be consumed by people. However, an earlier OTA report entitled Cancer Testing Technology and Saccharin (7) found that "animal tests are the best current methods for predicting the carcinogenic effect of substances in humans. All substances demonstrated to be carcinogenic in animals are regarded as potential human carcinogens; no clear distinctions exist between those that cause cancer in laboratory animals and those that cause it in humans. The empirical evidence overwhelmingly supports this hypothesis."

The report also found that the rationale for feeding high doses was sound.

The rationale for feeding large doses of a substance in animal tests is as follows. As the dose of a substance that causes cancer is increased, the number of exposed animals that develop cancer also increases. To conduct a valid experiment at high dose levels, only a small number of animals (perhaps several hundred) is required. However, to conduct a valid experiment at low dose levels, a very large number of animals is required. (The smallest incidence rate detectable with 10 animals is 10 percent or 1 animal. To detect a 1-percent incidence rate, several hundred animals would be required.) Another important variable is the strength of the carcinogen. The stronger the carcinogen, the greater will be the number of animals getting cancer at a particular dose (7).

If data from a carcinogen bioassay are available at the time an environmental contaminant is discovered in food, the results provide crucial information to guide the regulatory decision. However, if no data or inadequate data exist, a newly commissioned carcinogen bioassay would require 2 to 3 years for completion. In this case, the carcinogen bioassay could be used to revise an initial action level.

\section{Short-Term Tests for Mutagenesis and potential Carcinogenesis}

Chemicals are evaluated for mutagenicity not only to detect potential carcinogens but also to detect the serious hazard posed by mutagens. In the long run, chemical compounds causing mutations and teratogenic effects may create a greater burden on society by increasing the incidence of genetic disease and birth defects than by causing cancer $(2,11)$.

A number of short-term tests and batteries of tests have been developed to assay chemicals for mutagenicity $(1,2)$. Many of the same tests are also viewed as screens for carcinogens on the assumption that cancer arises following damage to the genetic material of cells (2). The ability of these tests to detect carcinogens is currently undergoing validation (8). The Environmental Protection Agency (EPA) currently uses some of these tests to identify mutagens $(9,10)$. Presently there is no universal agreement on a "best" set of tests.

Environmental contaminants in food are screened to identify those capable of causing genetic damage, to determine the types of damage they cause, and to evaluate the risks they pose to the general population and certain subpopulations.

The major difficulty in designing tests to detect mutagens and potential carcinogens is the need to reflect the metabolic capabilities of man as nearly as possible while remaining economically realistic. Whole animal tests more nearly reflect responses of man and are therefore useful in estimating risk to humans. Tests using lower organisms are less directly applicable to humans, but they are simpler and more economical to perform. As a consequence they are more popular for use in mutagen screening than animal tests. Risk estimates based on tests in lower organisms have been proposed (12) but are not accepted as sufficient evidence for setting action levels and tolerances.

Many chemicals are not directly mutagenic, but once ingested or absorbed are converted into mutagenically active derivatives. Activation therefore poses important practical and conceptual problems that must be dealt with in evaluating the mutagenic potential of environmental agents. The practical problem is one of designing tests to identify 
mutagens that adequately mimic the metabolic capabilities of an intact animal. The conceptual problems focus on the fact that evidence of an agent's mutagenicity is not sufficient to evaluate the actual risk posed to an individual or a population by exposure to the agent (2).

The ability of an agent to cause genetic damage in an intact animal depends not only on the mutagenic potential of the agent or its metabolizes but on a number of other factors as well. The fate of any chemical substance entering the body is determined by a dynamic process involving its absorption, distribution, biochemical alteration, and excretion. Many mutagenic agents are formed by the action of intestinal bacteria. Consequently, the route of exposure to an agent can play a significant role in determining its activity. Enzymatic processes in the body inactivate as well as create mutagens. Many active mutagens may exist in the body as intermediates in the metabolic pathways that process the parent compounds. As a result, the active mutagen may have a short lifespan and be distributed only in those tissues that possess high levels of activating enzymes (2).

There are four major factors determining the ability of an environmental agent to produce a genetically adverse effect in an organism, The first is the metabolic response of the organism to the agent. This response will determine the distribution, lifespan, and fate of the mutagen in the body tissue. The second is whether the mutagen damages the genetic material in cells. The third is the response to the genetic damage by the DNA repair machinery in the cell. Fourth is the type of lesion the agent is capable of producing in the genetic material.

Short-term tests for detection of mutagenesis and potential carcinogenesis fall into four categories:

- procedures that can be carried out directly in human populations,

- intact animal tests,

- tests employing cultured mammalian cells, and

a tests employing micro-organisms.
Several procedures that can be carried out directly in exposed human populations have been found to be indicators of the presence of mutagenic agents. These procedures include analysis of white blood cells for chromosome aberrations (cytogenetic analysis) (13), analysis of blood and/or urine of the presence of mutagenic agents (14), analysis of semen for abnormal sperm (15), and the detection of extra chromosomes in sperm (16).

Intact animal tests usually use as test animals either mice or fruit flies [Drosophila melanogaster). The dominant lethal test detects lethal damage resulting primarily from chromosomal aberrations by treating male mice with test agents and mating them. The uteri of the pregnant females are examined for fetal death and resorption. Because the endpoint is detected in the offspring of the treated animal, mutation in the germinal cells (the sperm) is detected by this method. The specific locus test involves the crossing of two genetically distinct strains of mice with visible markers, such as spotted coats. Mutations are detected by the appearance of recessive traits in the offspring. The utility of this testis restricted because of the number of animals needed. Cytogenetic screening of white blood cells or bone marrow cells from treated animals detects damage to chromosomes. The micronucleus test detects chromosome breaks in bone marrow cells of treated animals (17).

The use of cultured mammalian cells for mutagenesis testing is a great simplification over the use of intact animals. At the same time, the cells retain much of the mammalian metabolism and genetic characteristics. Moreover, human cell cultures can be used where exposure of people to toxic agents could not be allowed. The major weakness of cultured mammalian cells as test systems is that they are isolated from the metabolic activity of the intact animal (2).

Several strategies have been developed to try to approximate the metabolic capabilities of the intact animal in mammalian cells, The simplest and most widely used activation system employs homogenates of mammalian 
liver. Either the liver homogenate is incubated with the test substance before it is applied to the cell culture, or the test substance and the homogenate are included in the test plates. A major difficulty with the liver microsome activation system is that it cannot be prepared with reproducible enzyme levels from one batch to the next. Storage conditions also alter the activities of the preparations, Furthermore, not all the activation mechanisms present in the intact animal are represented in the liver homogenate. The greatest advantage of the liver homogenate method is that it preserves the simplicity of in vitro testing (18).

Methods for mutagen screening have been developed using many micro-organisms, including fungi, bacteria, and viruses present in bacteria. The most widely known system is the "Ames test" which employs specially bred strains of Salmonella bacteria. Figure 5 illustrates the steps in the Ames spot test. The Ames plate test allows quantitative comparisons to be made of mutagenic potential (19). Numerous other tests have been devised which are in varying stages of validation (20). Some of these are described in appendix C.
The great appeal of these techniques is that they are rapid, simple, and inexpensive. No animal colony is required, and the necessary technical skills are those required for conventional bacteriology.

These tests have been criticized because the cell structure of bacteria is very different from the cell structure of mammals. Validation studies now underway are attempting to evaluate 30 mutagenicity assay systems for their ability to predict chemical carcinogenicity (8).

Bacteria also lack the enzyme systems that are the principal mammalian mutagen-activating enzymes. This criticism is partially overcome by the use of liver homogenates described above. Finally, each test is sensitive to one particular type of genetic damage. Therefore, the best approach is to use a battery of short-term tests designed to test for the different types of genetic damage.

\section{Interactions of Two or More Substances}

Tests designed to detect effects from interactions of two or more substances are of lim-

Figure 5. - Steps in the Ames Spot Test

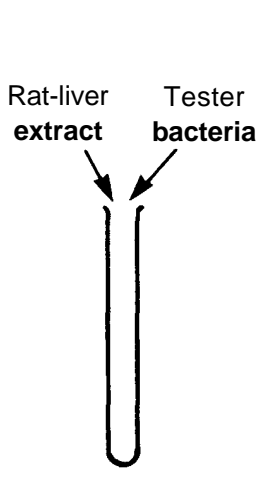

MUTAGENESIS is detected in the Ames test by mixing an extract of rat liver (which supplies mammalian metabolic functions) with tester bacteria (which cannot grow because a mutation makes them unable to manufacture histidine, a necessary nutrient) and plating the mixture on an agar medium so that a thin layer of bacteria covers the medium evenly, as is shown on a microscopic scale (1). In this "spot

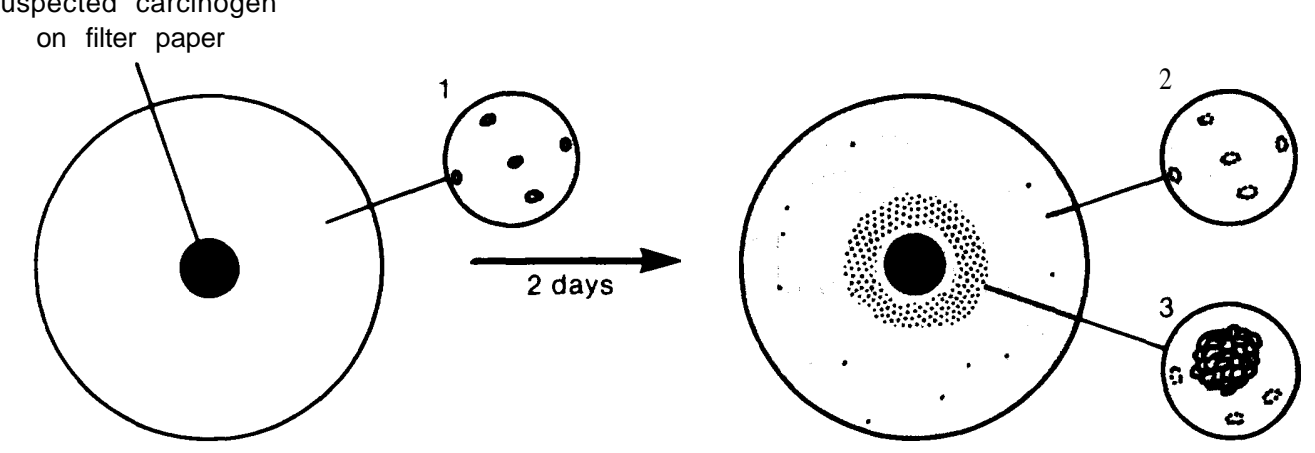

assay" a dose of the chemical to be tested is placed on a disk of filter paper on the tester bacteria. After 2 days most of the his bacteria have died for lack of histidine (2), but DNA damage caused by the chemical diffusing out from the disk has given rise to mutations, some of which result in reversion of the his ${ }^{-}$mutation. The histidine-making revertant bacteria proliferate, forming visible colonies (3). 
ited use for regulatory actions. This does not mean that such information is not important. It rather reflects the rudimentary nature of our present understanding of interactions, the complexities of the tests, and the difficulties in interpreting the results in a fashion meaningful to regulators.

Six different types of interactions may occur when two or more chemicals interact in a biological system. The effects produced may differ in magnitude from those caused by any one of the chemicals alone.

- If the interaction of two substances produces an effect equal to the sum of the individual effects, the response is called summation or addition.

- If the interaction of two substances produces an effect greater than would be anticipated from the sum of the individual effects, the response is known as potentiation or synergism.

- If the effect is less than the sum of the two would predict, the response is antagonism.

- When an inert substance, having no observed effect at a given dose, enhances the effect of another simultaneously administered chemically, the effect is usually referred to as activation.

- If an inert substance decreases the effect of another chemical administered simultaneously, the effect is called inhibition.

- Finally, there may be no interaction and each chemical would exert its own effect independently of the other (2 1).

Once ingested, a chemical may exert an action locally in or on the stomach or intestines. While such effects possibly could be produced by environmental contaminants, they are not likely to be observed at the low levels normally encountered in food. The more serious concern is the systemic effects that may occur after absorption from the gastrointestinal tract.

Following absorption from the gastrointestinal tract, additive chemicals may produce their effect by acting on a target organ, by acting on different target organs or systems, or by acting differently on different organs to produce the same effect. Most of the interactions are at the biochemical level and the mechanisms are still being studied.

The key consideration is whether present testing technologies are adequate to provide data that are useful in making regulatory decisions. Techniques have been developed to study the interactions of drugs, other chemicals, and physical agents such as radiation. Such techniques should be applicable to the study of the effects of environmental contaminants. However, their scope is usually limited to the study of two component interactions because of the large number of test animals required and the difficulties in interpreting the results. For example, a relatively simple test involving two agents in a factorial design would require about 100 animals. A more recent study (22) of the combined effects of cadmium, mercury, and lead used 50 to 60 animals in each of 15 different treatment groups. The results indicated that a particular combination of the three metals could be synergistic, antagonistic, or additive, depending on the relative doses employed (table 13).

Table 13. - Combined Effects of Cadmium (Cd), Mercury $(\mathrm{Hg})$, and Lead $(\mathrm{Pb})$

\begin{tabular}{|c|c|c|c|}
\hline \multirow{2}{*}{$\begin{array}{l}\text { Metal } \\
\text { combinationsa }\end{array}$} & \multicolumn{3}{|c|}{ Interaction } \\
\hline & Antagonism & Addition & Synergism \\
\hline $\begin{array}{l}\mathrm{Pb} / \mathrm{Hg} \ldots \ldots \\
\mathrm{Hg} / \mathrm{Pb} \ldots \ldots \\
\mathrm{Pb} / \mathrm{Cd} \ldots \ldots \\
\mathrm{Cd} / \mathrm{Pb} \ldots \ldots \\
\mathrm{Hg} / \mathrm{Cd} \ldots \ldots \\
\mathrm{Cd} / \mathrm{Hg} \ldots \ldots \\
(\mathrm{Pb}+\ldots \ldots \ldots \\
(\mathrm{Hg}+\ldots \ldots \\
(\mathrm{Pb}+\mathrm{Pb}) / \mathrm{Cd}) / \mathrm{Cd} \ldots \ldots \\
(\mathrm{Cd}+\mathrm{Pb}) / \mathrm{Hg} \ldots \ldots \\
(\mathrm{Hg}+\ldots \ldots \\
(\mathrm{Cd}+\mathrm{Cd}) / \mathrm{Pb} \ldots \ldots \\
(\mathrm{Hg}) / \mathrm{Pb} \ldots \ldots\end{array}$ & 0.64 & 1.0 & $\begin{array}{r}1.4 \\
18.4 \\
1.7\end{array}$ \\
\hline \multicolumn{4}{|c|}{$\begin{array}{l}\text { For each combination. the metal or metals in the numerator were held con- } \\
\text { stant at or near the essentially no effect level (LD } 1 \text { ). The dose of the metal in } \\
\text { the denominator was increased to obtain a dose-response curve. When a pair } \\
\text { of metals appear in the numerator, the first was fixed at its LD } 1 \text { and the sec. } \\
\text { ond was increased to obtain an LD } 1 \text { for the pair. } \\
\text { OInteractions are expressed as the ratio of the dose of the increased metal } \\
\text { alone needed to attain the LD } 50 \text { to the corresponding value for that agent in } \\
\text { the presence of a single metal or a metal pair. If the ratio is greater than } 1 \text {. syn. } \\
\text { ergism has occurred. If the ratio is equal to } 1 \text {, the interaction is additive. If the } \\
\text { ratio is less than } 1 \text {, the interaction is antagonistic. }\end{array}$} \\
\hline $\begin{array}{l}\text { SOURCE: Adapted from J. Sc } \\
\text { fects in Toxicolog } \\
\text { mium, Mercury, and } \\
\text { Health 4:763.1978. }\end{array}$ & $\begin{array}{l}\text { chubert. E. J. Riley } \\
y \text {-A Rapid Syste }\end{array}$ & $\begin{array}{l}\text { and S. A. Tyl } \\
\text { matic Testing }\end{array}$ & Procedure: Cad- \\
\hline
\end{tabular}


The testing for interactions among toxic substances is further complicated by the necessity to limit the number of substances studied, Because of the large number of animals required, and the difficulties in interpreting results, the number of substances tested are confined to those that may be most important, Deciding what is "important" to study out of the vast number of toxic substances to which we are exposed is a difficult problem, Perhaps a reasonable approach would be to focus on those to which people are known to be exposed (see chapter VII). The choice could also be based on structureactivity relationship or known mechanism of action, Chemical selection for testing is being reviewed and evaluated in an ongoing OTA study entitled "Assessment of Technologies for Determining the Carcinogenic Risk from the Environ merit."

The study of the interactions of two or more substances is an area in which far more basic research is required before meaningful information can be generated for regulatory decisions. At the present time, no satisfactory methods exist for testing the interactions of more than two chemicals.

\section{Extrapolating From High Doses to Low Doses}

Quantitative risk assessment. based on mathematical models, is often proposed as an alternative to the current use of safety factors (chapter 111) $(24,25)$. This approach is now most extensively employed in assessing carcinogenic risks (26). The technique is also being investigated as a means to evaluate other types of irreversible toxic effects (23). The following discussion of mathematical extrapolation of risk involves only its uses in determining risks from chronic low-level carcinogenic insults.

Mathematical models can be used to estimate the number of extra cancers that are likely to be caused by the presence of a carcinogen in the food supply. Models may be used to estimate a tolerance based on calculations of a "safe dose" for human consumption. They can also forecast the likely change in the number of extra cancers that would accompany some projected increase or decrease in the level of human exposure occurring either as a result of regulatory action or inaction, The technique is therefore used to calculate benefits to be derived from reducing human exposure to a substance.

The typical carcinogen bioassay uses around 100 animals at each experimental dose. If a particular experimental dose produces evidence of a lifetime increase in cancer of 1 in 10, this increase can be measured using 100 animals. But if the increased cancer risk is less than 1 in 100, this increase will often not be detected even with a 100animal feeding experiment. The extra human risk resulting from environmental exposure is usually much smaller than 1 in 100 (perhaps on the order of 1 in 100,000) for any given chemical over a lifetime exposure. It would not be practical to conduct an experiment with enough animals to directly measure this small an increase in risk (30).

For these reasons, carcinogen bioassays use (in addition to a control dose of zero) several doses at which the projected extra cancer incidence may be 1 in 10 or larger. These high-dose data are then used to estimate the extra risk at a very low dose where the extra risk may be no larger than 1 in $1,000,000$. These problems are often referred to collectively as the "low close extrapolation problem" (30).

A low-dose extrapolation involves the choice of a mathematical function to model the dose-response relationship and the choice of statistical procedures to apply to the mathematical function (24-29). The choice of mathematical function is crucial to the outcome of a low-dose risk estimate. If the assumed relationship between tumor occurrence and dose does not apply in the low-dose regions to which the extrapolation is being made, a serious overestimate or underestimate of the "safe dose'" may result. Even though different dose-response models may agree in the observable response range, they could differ by many orders of magnitude at low-dose levels. Furthermore, there is no experimental way to 
predict the shape of the dose-response curve at very low doses,

Because of the possible disparity of doseresponse functions when extrapolated to low doses, the dose-response function must be selected not only on the basis of how well it can be made to fit experimental data but also on the basis of known (or at least plausible) information on the biological mechanisms through which a chemical induces or promotes cancer. This is a major source of uncertainty in extrapolation procedures, since the exact biological mechanisms through which carcinogenesis may occur are unknown (30).

Several theories on the nature of the process by which cancer develops serve as the bases for different low-dose extrapolation models. Some "linear" models project that the relationship between the dose received and the cancer risk is a straight line at low doses and that there is no threshold. Other models level off at low doses and therefore predict that a threshold dose exists below which the agent has no carcinogenic effect (24-30).

Newer models take into account the effects of metabolic activation and detoxification upon carcinogenic dose response (26), These "kinetic" models encompass free toxic substances, metabolizes, deactivators, and the possible interactions of these substances, Only a "steady-state" situation is studied in that the variation over time of the concentrations of these agents is not considered. If deactivation of the carcinogen is 100-percent efficient, the model predicts a threshold dose below which there is no carcinogenic risk. However, in a naturally occurring process, it is likely that deactivation would be less than 100-percent effective. A number of modifications to the model allow for imperfect deactivation. These situations rule out a threshold and would lead directly to a model for which carcinogenic response varies linearly with dose at low dose,

The mechanisms through which most carcinogens produce cancer are not sufficiently understood so that the shape of the dose- response curve can be predicted with certainty, As pointed out earlier, experiments of sufficient size to permit direct experimental investigations of the dose-response curve at low dose cannot be conducted. There are plausible arguments that the dose-response curve is linear at low dose for many carcinogens, On the other hand, no mechanism that would lead to a more cautious dose-response relationship has been seriously proposed except for some dose-response relations in radiation-induced carcinogenesis.

In view of these uncertainties, it would seem reasonable to base estimates of added cancer risk on a mathematical model that encompasses low-dose linearity until the mechanisms through which the carcinogen operates are understood sufficiently to conclusively rule out low-dose linearity,

Caution must be used in interpreting the results of low-dose extrapolations, particularly when they are applied to humans, Table 14 demonstrates that different models applied to the same data sets yield differing estimates of virtually safe doses. Virtually safe doses based on the multistage model are identical with those based on the one-hit model when there is only one experimental dose. This is illustrated with Data Sets I and

\section{Table 14.-Virtually Safe Doses Computed From Three Different Data Sets and Three Different Models}

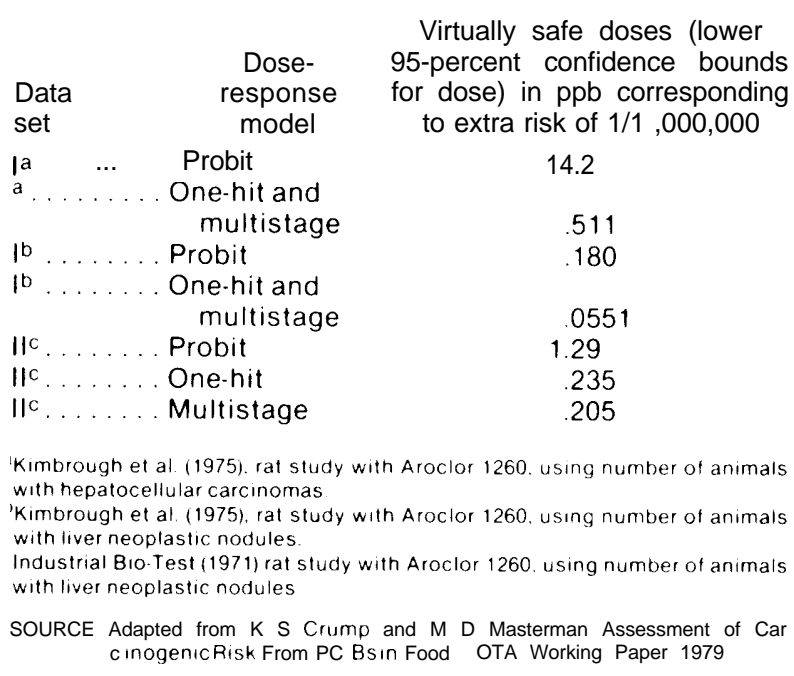


II. The multistage model also yields virtually the same value as the one-hit model for Data Set III. However, as explained in appendix D, the multistage model can yield higher values for the virtually safe dose than the one-hit model whenever the data exhibit upward curvature and are inconsistent with the one-hit model (30).

Caution also must be exercised when comparing calculations of extra lifetime risks. Table 15 summarizes the results of an FDA risk assessment of PCBs (31) with a similar risk assessment commissioned by OTA (30). Differences can arise in such calculations when different methods are used to extrapolate from animals to man. The extrapolation can be performed on the basis of milligrams per kilogram of body weight per day, parts per million of substance in the diet, milligrams of substance per square centimeter of body surface area per day, or milligrams of substance per kilogram of body weight per lifetime.

Table 15. - Extra Lifetime Risks of Cancer Associated With Consumption of PCBs in Food

\begin{tabular}{|c|c|c|}
\hline Dose (g/day) & $\begin{array}{l}\text { Extra lifetime } \\
\text { risk/100,000 }\end{array}$ & $\begin{array}{l}\text { Upper limit of new } \\
\text { cancers/year }\end{array}$ \\
\hline \multicolumn{3}{|l|}{ FDA' } \\
\hline 9 2' & 44 & 21 \\
\hline $149^{\prime}$ & 72 & 34 \\
\hline & \multicolumn{2}{|c|}{$\begin{array}{l}\mathrm{OTA}^{\mathrm{b}} \\
33(!\end{array}$} \\
\hline $33(!$ & 013 & 4 \\
\hline $3.7^{e}$ & 035 & 11 \\
\hline $127 f$ & 5 & \\
\hline \multicolumn{3}{|c|}{ 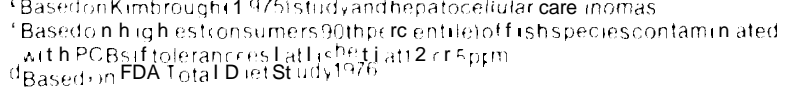 } \\
\hline \multicolumn{3}{|c|}{ 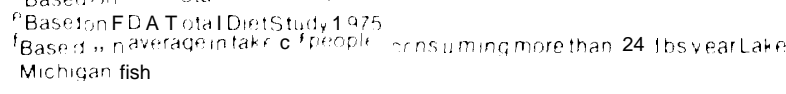 } \\
\hline
\end{tabular}

Differences also arise when varying animal data serve as the basis for the risk assessment, And, of course, the choice of the model also affects the final outcome. In this case, both extrapolations were based on milligrams of PCB per kilogram of body weight per day. Both FDA and OTA applied a linear model: however, different assumptions were made on the amount of PCBs that would be ingested and on the size of the exposed population. FDA's risk assessment therefore applies to the 15 percent of the total U.S. population expected to consume the fish species known to be most highly contaminated with PCBs. The OTA calculations are based on the average daily intake of PCBs based on FDA's total tiet study $(3.3$ and $8.7 \mu \mathrm{g} / \mathrm{day})$ and on estimates of the $\mathrm{PCB}$ intake of people who consumed more than $24 \mathrm{lbs}$ of Lake Michigan sport fish per year.

Although many different dose-response models exist and can be used for low-dose risk assessment, it appears prudent at this time to use a model that does not arbitrarily rule out low-dose linearity. Models are still being developed and when" appropriate could be used to guide regulatory decisions. Doseresponse models for low-dose risk assessment provide a useful technique for assessing the possible added risk attributable to environmental contaminants. Such models also might well be used in place of safety factors in future procedures adopted for food contaminant regulations. The major impediment to the widespread use of the models in environmental food contamination considerations is the lack of data on which to perform the analysis.

\section{CHAPTER V REFERENCES}

1. SRI International. "Assessment of Methods for Regulating "Unavoidable" Contaminants in the Food Supply," OTA Working Paper, 1978.

2. Legator. M. S. "Short Term Procedures for Determining Mutagenic/Carcinogenic Activitv," OTA Working Paper, 1979.

3. MacMahon, B., and T. F. Pugh. Epidemiology Principles and Methods. Little, Brown and
Company, Boston, Mass., 1970.

4. Ray, V. "Are Benzene Effects Limited to the Chromosomal Level?, " Banbury Report 1Assessing Chemical Mutagens: The Risk to Humans, Cold Spring Harbor Laboratory, 1979.

5. Jackson, R. S. "The Limitations of Applying Human Epidemiology to the Regulator Pro- 
cess for Toxic Substances, Particularly Carcinogens," OTA Working Paper, 1979.

6. Sontag, J. M., N. P. Page, and U. Saffiotti. Guidelines for Carcinogen Bioassay in Small Rodents, DHEW Publication No. (NIH) 76-801, Government Printing Office, Washington, D. C., 1976.

7. Office of Technology Assessment. Cancer Testing Technology and Saccharin, Government Printing Office, stock no. 052-00300471-2, Washington, D. C., 1977.

8. U.S. Department of Health, Education, and Welfare. National Toxicology Program Annual Plan Fiscal Year 1979.

9. U.S. Environmental Protection Agency. FIFRA Testing Guidelines, 1978.

10. U.S. Environmental Protection Agency. TSCA Test Standards, 1979.

11. Neel, J. V. "Mutation and Disease in $\mathrm{Hu}-$ mans, "Banbury Report 1-Assessing Chemical Mutagens: The Risk to Humans, Cold Spring Harbor Laboratory, 1979.

12. Committee 17, Council of the Environmental Mutagen Society, "Environmental Mutagen Hazards," Science 187:503, 1975.

13. Kilian, D. J., and D. Picciano. "Cytogenetic Surveillance of Industrial Populations," Chemical Mutagens, Principles and Methods for Their Detection, Vol.IV, A. Hollaender (cd.), Plenum Press, New York, 1976.

14. Legator, M. S., L. Troung, and T. H. Connor. "Analysis of Body Fluid Including Alyklation of Macromolecules for Detection of Mutagenic Agents," Chemical Mutagens, Principles and Methods for Their Detection, Vol. V, A. Hollaender and J. DeSerres (eds.), Plenum Press, New York, 1978.

15. Heddle, J. A., and W. R. Bruce. "On the Use of Multiple Assays for Mutagenicity, Especially the Micronucleus, Salmonella, and Sperm Abnormality, Assays in Progress, "Genetic Toxicology, P. Scott, B. A. Bridges, and F. H. Sobels (eds.), 1977.

16. Kapp, R. A. Mutation Research, in press, 1979.

17. Kilbey, B. J., M. Legator, W. Nichols, and C. Ramel. Handbook of Mutagenicity Test Procedures. Elsevier, Amsterdam, 1977.

18. Ames, B. N., W. E. Durston, E. Yamasaki, and F. O. Lee. "Carcinogens and Mutagens: A Simple Test Combining Liver Homogenates for Activation and Bacteria for Detection," Proceedings of the National Academy of
Sciences 70:2281, 1973

19. Ames, B. N. "A Bacterial System for Detecting Mutagens and Carcinogens, " Mutagenic Effects of Environmental Contaminants, H. E. Sutton and V. I. Harris (eds.), Academic Press, New York, 1972.

20. Devoret, R. "Bacterial Tests for Potential Carcinogens, "Scientific American 241(2):40, 1979.

21. Fingl, E., and D. M. Woodbury. "General Principles," The Pharmacological Basis of Therapeutics, L. S. Goodman and A. Gilman (eds.), 5th edition, MacMillan Publishing Co., Inc., New York, 1975.

22. Schubert, J., E. J. Riley, and S. A. Tyler. "Combined Effects in Toxicology-A Rapid Systematic Testing Procedure: Cadmium, Mercury, and Lead, "Journal of Toxicology and Environmental Health 4:763, 1978.

23. Van Ryzin, J., personal communication, 1979.

24. Interagency Regulatory Liaison Group, Work Group on Risk Assessment. "The Scientific Bases for Identification of Potential Carcinogens and Estimation of Risks," Journal National Cancer Institute 63:241, 1979.

25. Scientific Committee of the Food Safety Council. "A Proposed System for Food Safety Assessment ,“ Food and Cosmet. Toxicol. 16, Supplement 2, 1978.

26. Cornfield, J. "Carcinogenic Risk Assessmerit," Science 198:693, 1977.

27. Crump, K. S., D. G. Heel, C. H. Langley, and R. Pete. "Fundamental Carcinogenic Processes and Their Implications for Low-Dose Risk Assessment, " Cancer Research 36: 2973, 1976.

28. Guess, H. A., K. S. Crump, and R. Pete. "Uncertainty Estimates for Low-Dose-Rate Extrapolations of Animal Carcinogenicity Data," Cancer Research 37: 3475, 1977,

29. Rai, K., and J. Van Ryzin. "Risk Assessment of Toxic Environmental Substances Using a Generalized Multi-Hit Dose Response Model," Energy and Health, N. Breslow and A. Whittemore (eds. ) SIAM Press, Philadelphia, Pa., p. 94, 1979.

30. Crump, K. S., and M. D. Masterman. "Assessment of Carcinogenic Risk from PCBS in Food," OTA Working Paper, 1979.

31. U.S. Food and Drug Administration. An Assessment of Risk Associated with the Human Consumption of Some Species of Fish Contaminated With Polychlorinated Biphenyls (PCB's). 1979. 
Chapter VI

\section{Methods of Estimating and Applying Costs to Regulatory Decisionmaking}




\section{Methods of Estimating and Applying Costs to Regulatory Decisionmaking}

In the previous chapter, various testing methods for assessing health risks were evaluated for their usefulness in regulating environmental contaminantsi.e., setting an action level or tolerance. The primary issue involved in assessing health risks is not whether the potential risks from an environmental contaminant should be evaluated for purposes of regulation but rather what testing methods are most appropriate for assessing potential risks?

The situation is reversed, however, when the associated costs of an action level or tolerance for an environmental contaminant are assessed. The primary issue is whether the costs should be taken into account in the setting of a tolerance or action level. This is a policy issue which is addressed in chapter IX, "Congressional Captions." In this chapter, the various approaches and techniques for estimating the costs and benefits of a proposed tolerance or action level are assessed, along with two common methods for applying cost and human risk data in the regulation of environmental contaminants. A more detailed discussion of the approaches and techniques for estimating the costs and benefits is provided in appendix E.

Two methods, the cost-effectiveness method and the cost-benefit method, are analyzed for their strengths and weaknesses as regulatory decision-assisting tools. This analysis provides a basis for the discussion in chapter IX on the role of economics in regulating environmental contaminants in food.

\section{COST-EFFECTIVENESS}

Cost-effectiveness is a regulatory decisionassisting tool that compares the estimated net costs of the proposed regulation in dollar terms with estimated reduction of human risk expressed in scientific terms. In the regulation of environmental contaminants, the costeffectiveness method would compare the estimated change in costs of a proposed action level or tolerance for a contaminant in food with the associated reduction in human health risk-i.e., the benefits of the proposed tolerance or action level. The comparison of net costs and risk reduction is performed for several alternative levels in order to select a tolerance or action level. The principal cost of regulatory action is the cost of food held off the market because it exceeds the proposed action level or tolerance. There would also be a reduction in medical costs as a result in the reduction of human risk. These costs would need to be subtracted from the cost of restricted food.

The cost in lost food would also have associated distributional effects which would need to be identified to fully weigh the impact of a regulation. These effects would include identifying the consequences for producers, change in the cost of food to consumers, and indirect impacts on such businesses as food processors, animal feed manufacturers, or bait and tackle shops,

The cost of food losses and related effects would be compared with the estimated reduction of human risk for the contaminant at the proposed action level or tolerance. The hu- 
man risk data could include such information as the reduction in the estimated number of new cancer cases, the potential mutagenic and teratogenic effects, and other toxicological effects described in chapters II and V. The toxicological effects vary by substance and are stated in scientific terms. The estimated net cost and reduced human risk at various proposed action levels or tolerances are estimated and compared. The cost-effectiveness method then requires the regulatory agency to make its own judgment on what is the proper balance of net cost and reduced human risk when setting a tolerance or action level.

The Food and Drug Administration (FDA), for example, followed a similar procedure in its decision to reduce the tolerance for polychlorinated biphenyls (PCBs). In deciding on a 2 parts per million ( $\mathrm{ppm}$ ) tolerance for fish, FDA compared for $5 \mathrm{ppm}, 2 \mathrm{ppm}$, and $1 \mathrm{ppm}$ in fish the estimated reduction of human risk with the estimated increase in the amount and cost of food expected to be taken off the market. Table 16 shows the human risk from cancer and cost data on commercial fish for each of the three levels and the net changes in risk and cost for moving to a 2-ppm and 1ppm tolerance. It is these net changes that are evaluated in the cost-effectiveness method, Though FDA reviewed other available toxicological data, it was FDA's judgment that the reduction in risk (13.3 cancers) for a 1-ppm tolerance did not offset the increased cost (\$10, 3 million) and that a proper balance for the net changes was established at $2 \mathrm{ppm}$.
Thus, FDA has placed an implicit dollar value on life. A more thorough application of the cost-effectiveness method would attempt to identify all the net changes in the benefits (the reduction of all known risks) and all known costs,

Judgment is an important aspect to the cost-effectiveness method. It allows the decisionmaking body, in this case FDA, flexibility in interpreting health data and evaluating the implications of that data on the setting of a tolerance or action level. This flexibility can be particularly important in the assessment of insufficient or variable toxicological data. While an agency's interpretation of toxicological data might differ from others and generate disagreement about the final decision, the cost-effectiveness method does allow the complexity and shades of gray to be factored into the decisionmaking process,

The health data used in the cost-effectiveness calculations are generated by the toxicological and epidemiological procedures reviewed in the previous chapter. There are also various approaches and techniques used to estimate the costs of food likely to be taken off or restricted from the market as a result of a proposed tolerance or action level. The cost estimates will vary depending on the approaches or techniques employed. All these approaches require chemical analysis of a statistically significant number of food products known or thought to be contaminated, With such data, the amount of contaminated food that exceeds the proposed action level or tolerance can be estimated,

Table 16._- Impact of PCB Tolerance

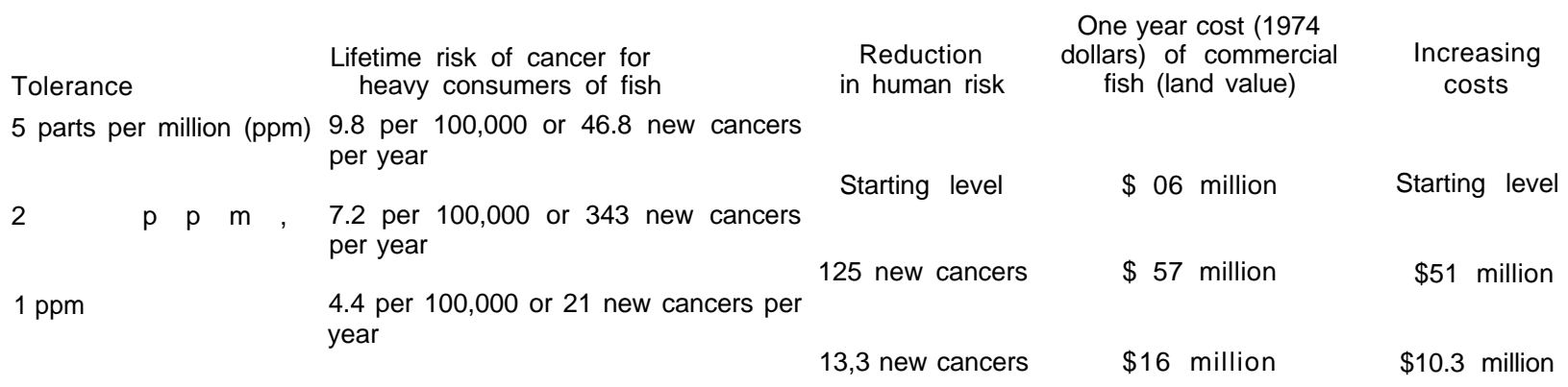


FDA multiplies the estimated amount of food removed from commerce by the appropriate price (production, wholesale, retail, etc. ) per unit (pound, bushel, ton, or animal, etc.). This method of estimating food costs is not the most accurate way of estimating costs and does not attempt to measure the indirect costs or distributional effects that result from an action level or tolerance.

There are two other approaches for estimating the costs from the reduction of the food supply: the alternative-cost approach and the opportunity-cost approach. The alternative-cost approach estimates the cost of the next best alternative method or methods, if available, for replacing food removed from the market.

For example, assume that $164,000 \mathrm{lbs}$ of lake trout in Lake Superior were restricted from commerce because of the 2-ppm tolerance for PCBs. The alternative-cost approach would require cost estimates of each available method as well as various combinations of methods for producing (and thus replacing) the entire $164,000 \mathrm{lbs}$ of lake trout, the amount condemned by the tolerance. In establishing the replacement cost, this approach does not consider price or supply shifts in the restricted food product nor shifts in the supply of other food products. Such shifts are likely to occur when large amounts of food in relation to the total supply are taken off the market. Consequently, this approach can lead in some instances to an overstatement of costs.

The opportunity-cost approach does include shifts in supply. But what information is included in the analysis depends on which techniques are used. The budgeting technique only includes shifts in supply for the contaminated commodity. This technique uses data on the production of the contaminated product to calculate the costs of producing and consequently replacing a particular amount of that food product that has been restricted from commerce. It is assumed that other commodities will be produced in the same amounts and at the same price that prevailed before the establishment of the tolerance or action level.

The modeling technique, however, includes not only supply shifts but also resulting price shifts in both contaminated food and other food products. With this technique, mathematical models of the relevant portion of the economy are employed to trace shifts in supply, changes in the amount and price of various commodities, and other factors affected by the restriction on the use of a contaminated product.

These models can, if properly programed, project changes in the production location of particular crops as well as changes in the use of various production factors in each region of the country. Thus modeling can more realistically describe likely reactions in food production to the change created by a tolerance level. The result is a better estimate of the potential cost of lost or restricted food.

In attempting to project replacement costs, the opportunity-cost approach (using either budgeting or modeling techniques) more accurately estimates the true costs of a tolerance or action level then either the alternative-cost or the FDA approach.

The distributional effects of a tolerance or action level can be incorporated in the costeffectiveness method. But the opportunitycost approach can better generate these distributional effects. With either method these effects are most easily identified when the data are initially being collected. The information is important in determining who will bear the brunt of the costs, both directly and indirectly. The distributional effects flowing from human health risks can also be identified. The available risk data can be assembled to determine who bears most of the potential and actual risks. Thus the distributional effects of the net cost and the reduction of risk from a tolerance or action level can be compared (1). 


\section{COST-BENEFIT}

The cost-benefit method compares the estimated dollar costs of a proposed regulation with the estimated dollar benefits of reducing human health risks. The cost-benefit method of setting tolerance or action levels also requires the evaluation of several alternative levels. The differences in benefits and costs as one moves from one proposed regulatory level to the next are then compared. The tolerance or action level would be lowered until the costs (impact from food condemned) are greater than the benefits (reduction of human risks). When this level is reached, the economically efficient tolerance or action level will be the next higher level at which the benefits exceed the costs.

By representing the costs and benefits for a proposed action level or tolerance in dollars, cost-benefit analysis attempts to make the two sides of the ratio more comparable. This method recognizes, as does cost-effectiveness, that any proposed action level or tolerance will incur costs in terms of food taken off the market and benefits reflected in the reduction of either actual or potential health risks. As the level is lowered, costs increase and human health risks decrease.

Techniques of estimating costs are similar in the cost-benefit and cost-effectiveness methods with the exception that the reduced medical costs are included with the benefits. There are some ways of valuing benefits, though, which are unique to the cost-benefit method.

Though both methods rely on the available toxicological data, cost-benefit requires the conversion of all data into dollar values. This economic conversion is best accomplished when the data are expressed as the number of premature deaths per year avoided, the number of person-days lost to illness avoided, or the probability that some percentage of the exposed population would die prematurely or would lose a specified number of days from normal activity due to illness.
Two approaches are available for converting the risks into dollars: forgone earnings and willingness to pay. In the forgone-earnings approach the analyst places an explicit economic value on life in attempting to estimate the productivity lost as a result of illness or premature death caused by a contaminant in food. This approach, however, does not include associated health costs such as medical expenses incurred from illness. Including such costs is required in order to more accurately represent the total reduction of health costs-i.e., the benefits. The willingness-to-pay approach allows people affected by the regulation (rather than the analyst) to estimate how much they would be willing to pay to avoid a risk.

The forgone-earnings approach attempts to estimate the lost earnings of those individuals who are estimated to become ill or die prematurely because of the contaminated food (1). The estimated dollar value of the benefit will be determined by various techniques chosen or assumptions made by the analyst in converting the risk data to a dollar value. Discounting and earnings are two areas in which the analyst can influence the value of the benefit.

Since many of the benefits of a tolerance are not likely to be realized until 10,20 , or 30 years later, discounting is used to convert future dollar benefits into present dollars. Discounting is also used for estimating future costs. Discounting is required even if the future dollars are adjusted for the rate of inflation because a dollar spent now can be invested productively to yield a larger number of real dollars-i.e., inflation adjusted-in the future, For example, $\$ 100$ invested at 5percent interest becomes $\$ 105$ in one year. Discounting is the reverse: $\$ 105$ next year has a present value of $\$ 100$ when the discount rate is 5 percent. This means that $\$ 10,000$ worth of benefits that will occur 10 years from now would actually have a present value of $\$ 6,135$ if a 7 -percent discount rate is used, 
While most economists agree that future costs or benefits need to be discounted, they do not agree on the value of the discount rate. The rate can vary from I percent to as high as 15 percent $(\mathrm{z})$. The rate, however, has to remain constant for both the costs and the benefits. Obviously, the value of the rate will affect the estimate of benefits or costs, The lower the discount rate, the greater the dollar estimate in present dollars. Consequently, more weight would be given to the benefits and costs accrued in the future.

The estimate of the benefits also depends on whether gross or net earnings are used. Gross earning estimates include an individual's or a group's total wages or salaries. Net earnings consist of total wages or salaries minus the individual's or group's consumption. Obviously the gross earnings estimate will be greater than the net earnings estimate.

The willingness-to-pay approach is conceptually a more correct approach in that it asks the individual to place a dollar value on the reduction of associated risks from an environmental contaminant. This value is then used for placing a value on life itself. While conceptually correct, this approach does have some inherent problems, such as: 1) the capability of the questioned person to accurately understand the ramifications of the risk and 2) the individual's economic position. For example, an economically disadvantaged person might place a small value on risk not because the person feels the risk is of little or no concern but because that person cannot afford an increase in food prices. This approach is affected by the assumptions made by the public being surveyed.

\section{APPLICATION OF METHODS FOR REGULATION}

The use of the cost-benefit and cost-effectiveness methods for regulatory purposes is affected by the following factors: 1) FDA's interpretation of the Food, Drug, and Cosmetic Act, 2) the approaches and techniques for estimating the economic value of the costs and benefits, and 3) the inherent difference between the two methods.

As noted earlier, FDA interprets the Act as requiring it to weigh only the impact of the cost and the amount of food removed from commerce in the setting of the proposed tolerance or action level. FDA's approach for estimating this cost can be applied in either the cost-effectiveness or the cost-benefit method, but both of these methods can rely on alternative-cost or opportunity-cost techniques which more accurately estimate the cost incurred from condemned food. These other techniques also more accurately estimate the tolerance or action level's impact on availability y of food than the approach used by FDA.

Whichever technique is employed to estimate the costs, adequate information is needed on the amount of food likely to be contaminated. Such information was available for PCBs in fish, but it is not available for PCBs in milk, poultry, and eggs. This is because contamination of milk, poultry, and eggs is likely to occur as a result of industrial accidents. Such accidents are sporadic and therefore difficult to predict (e.g., the July 1979 PCB contamination of poultry and eggs in Idaho) (3). Consequently, the estimates for costs incurred because of food removed from commerce cannot be determined for such contamination incidents, and thus neither method can be employed. Both methods could be used to set a tolerance or action level for mercury and kepone in fish.

The approach and techniques used by the cost-effectiveness methods for generating necessary data can take considerable resources and time, 2 months to over a year to gather the data just for the costs alone. The amount of time needed depends on the approaches and techniques used. The more accurate the information being generated, the more time and resources are required. 
FDA, however, often has to make an initial decision in the form of an action level in 2 months or less for a newly identified environmental contaminant. A sufficient amount of time is usually available for utilization of the various approaches or techniques if a followup decision is involved. For example, 6 years expired from the time an initial PCB action level was proposed until a final tolerance for PCB was proposed this year. Either method is more likely to be used in setting a formal tolerance than an initial action level for an environmental contaminant.

The substantive difference between the cost-benefit method and the cost-effectiveness method is that the cost-benefit method places an explicit value on life by converting the health data to dollars while the cost-effectiveness method places an implicit value on life by weighing the health data in its scientific form with the costs. As a result, a significant amount of judgment is exercised by the analyst using the cost-benefit method when selecting the different approaches and techniques for estimating the benefits. As discussed earlier, the selection of these approaches and techniques has a strong bearing on the outcome of the ratio and consequently the tolerance established by this method.

The cost-effectiveness method places a greater judgment burden on the agency and less on the analyst. While the analyst does affect the outcome, judgment is primarily exercised by the agency in weighing the net cost with the reduction in human risk (benefits). The agency exercises less judgment in the cost-benefit method, which only requires a comparison of the numbers for each side of the ratio to establish the appropriate tolerance.

The cost-effectiveness method has the potential to reveal more of an agency's thinking in the decision than cost-benefit does. This was demonstrated with the earlier discussion of FDA's setting of a 2-ppm tolerance for PCBs in fish. In addition, because it recognizes the uncertainties inherent in the estimates of the health risks, the cost-effectiveness method allows FDA the flexibility to adjust the weight given to the benefits or the costs in its decision. For these reasons, the cost-effectiveness method is the more appropriate method at this time for weighing the costs in the setting of a tolerance.

Neither method can evaluate all the information required by FDA in setting a tolerance. For example, FDA requires for enforcement purposes analytical methodologies that can detect, measure, and confirm the identity of the contaminant at the level being proposed in food. This means that the tolerance cannot be set at a level below the available analytical capabilities for detecting the contaminant in food. While the analytical capability is an important factor in setting a tolerance, it cannot be evaluated within the decisionmaking framework by either method. Consequently, these two methods should be viewed as decision-assisting aids that allow the regulator the means to weigh many of the relevant costs and benefits in the setting of a tolerance.

\section{CHAPTER VI REFERENCES}

1. Epp, Donald J. “Economic Analysis of Alternative Action Levels in the Regulation of Environmental Contaminants of Food, " OTA Working Paper, April 1979.

2. Weinstein, Milton C., and William B. Stason. "Foundations of Cost-Effectiveness Analysis for Health and Medical Practices, "New England Journal of Medicine, Mar. 31, 1977.

3. Foreman, Carol Tucker, Assistant Secretary for Food and Consumer Services, U.S. Department of Agriculture. Testimony before the Subcommittee on Oversight and Investigations, House Interstate and Foreign Commerce Committee, Sept. 28, 1979. 
Chapter VII

Monitoring Strategies 


\section{Monitoring Strategies}

Monitoring involves the systematic collection and chemical analysis of food samples or other samples from the environment. The aim is to protect consumers by determining short- and long-term trends in the levels of various chemicals in food and the environment.

\section{STRATEGIES}

Monitoring strategies can be shaped to achieve either of two objectives. The first is to identify food lots that violate established tolerances and action levels. The second is to identify new environmental contaminants as they enter the human food chain.

The first objective is met by regulatory monitoring: the second through investigatory monitoring. Each of these strategies could be complemented by specimen banking. Neither is incompatible or mutually exclusive.

\section{Regulatory Monitoring}

The Federal agencies responsible for limiting consumer exposure to contaminated food now conduct regulatory monitoring. Food samples are collected and analyzed for environmental contaminants for which action levels and tolerances have been established. Based on available information or agreements with States, not all samples are analyzed for all regulated substances. Regulatory monitoring employs standardized, accepted analytica1 techniques. Because the procedures are standard and can be verified by other laboratories, they generate data that can be presented in courts of law with little probability of being successfully contested.

Chapter III reviewed Federal monitoring programs and chapter IV reviewed State monitoring programs. It is unlikely that these monitoring programs will detect new environmental contaminants, since both are restricted to searching for regulated chemicals.
Therefore, investigatory monitoring approaches are vital.

\section{Investigatory Monitoring}

Investigatory monitoring attempts to detect unregulated chemicals as they enter the food chain. This strategy involves the collection and analysis of samples which may or may not be foods, The analytical techniques employed for the detection of unregulated chemicals may or may not be accepted as standard methods comparable to those used for regulatory monitoring.

Analytical methods for investigatory monitoring include broad-spectrum determinations that may sacrifice some quantitative information (i. e., exactly how much of a given substance is present in a sample) for more qualitative information (i. e., better assessment of what or how many foreign substances are in the sample). These analytical methods are not necessarily designed for use in litigation, They are designed primarily to indicate the presence of a potentially hazardous substance. If one is found, an accepted analytical method to detect the substance would have to be developed-a method compatible with instrumentation existing in regulatory-monitoring laboratories.

Investigatory monitoring includes two discrete types of monitoring: monitoring for suspected environmental contaminants, and monitoring for uncharacterized environmental contaminants. Each of these (as well as 
regulatory monitoring) can be complemented by specimen banking.

\section{Monitoring for Suspected Environmental Contaminants}

Some chemicals that are not regulated by action levels or tolerances are suspected to be dangerous to humans if consumed in foods. This group includes chemicals that may be present in food because of their use, toxicity, production volume, and persistence. Examples of these chemicals can be found on the Environmental Protection Agency (EPA)/ Natural Resources Defense Council (NRDC) priority pollutant list established in June 1977. These substances may be called "suspected" or "potential" environmental contaminants.

Monitoring for suspected environmental contaminants involves a different strategy than the one used in monitoring for regulated contaminants. Under the latter strategy, foods are analyzed for compounds with specified action levels and tolerances to provide information for regulatory enforcement. This is

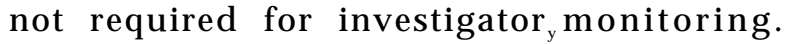
Thus, the monitoring program for suspected environmental contaminants is generally not as intensive (see chapter 111), Furthermore, the analytical methods for detecting suspected environmental contaminants may not be as prescribed as those for regulated contaminants.

Suspected environmental contaminants could be identified by surveying the universe of industrial chemicals and ranking them according to their potential for entering the food supply in toxic amounts (1). Such an approach has been recommended to FDA by an internal study group established by former Commissioner Donald Kennedy (7),

This method has been employed by the Food and Drug Administration (FDA) to develop a list of chemical contaminants in food, The criteria used in selecting the chemicals included: occurrence in food, volume of production, associated impurities or byproducts, predicted environmental stability, pattern of use, oil/water partition coefficients, bioaccumulation potential, known toxicity, and means of disposal. The FDA list of chemical contaminants in foods is shown in table 17.

This approach is limited by available analytical methods. Most of the chemicals recognized as food contaminants are those that are relatively easy to detect by gas chromatography or atomic absorption spectrometry. Chemicals that cannot be easily detected by these methods may, of course, remain unidentified and unrecognized as food contaminants (l).

The factors used to identify a chemical's potential for entering the food supply are based on knowledge of the properties and environmental behavior of other chemicals already known to be in food. This knowledge, in turn, is based on our information about the extent of contamination information that depends on our analytical capabilities. Thus, there is an inherent tendency to identify as potential food contaminants those chemicals that are similar to chemicals already identified in food. Such a tendency can only be offset by the use of good scientific judgment or the development of new data. This bias illustrates a general weakness in all systems for setting priorities: chemicals on which there is no information will automatically be given low priority unless some room is left for largely intuitive judgments (1). The scientific criteria and methods used in determining what priority various toxic substances receive in monitoring programs are discussed in more detail in appendix F.

Although these exercises in setting priorities suffer from many limitations (including lack of data, poor choices of criteria on which to set ranks, deficiencies in the scoring and ranking systems, and deficiencies in scientific judgment), they still can serve a valuable function in guiding monitoring systems. Setting priorities is a prescreening exercise in which a compromise is struck between the effort expended in preparing a priority list and the effort that would be wasted in identifying and quantifying all the chemicals present in a sample, 
Table 17. - Chemical Contaminants in Foods ${ }^{a}$

Jhemical contamınant

\section{Aromatic amines}

1.naphthylamine

V-ethyl-N-phenyl benzylamıne

$\checkmark$-ethyl-N-(m-tolyl) benzylamine

3.3'-dichlorobenzidene

3enzidene

\section{Triaryl phosphates}

Jydraul 50E

triphenyl phosphate

cumylphenyl diphenyl phosphate........

nonylphenyl diphenyl phosphate.

sopropylphenyl diphenyl phosphate.

rixylenyl phosphate

ricresyl phosphate

?-ethylhexyl diphenyl phosphate

shlorinated benzenes

nonochlorobenzene

1.4-dichlorobenzene.

1.2-dichlorobenzene

1.3.5-trichlorobenzene

1.2.4-trichlorobenzene

1.2,3-trichlorobenzene.

$1,2,3.5$ tetrachlorobenzene

1,2,4.5-tetrachlorobenzene

1,2,3.4-tetrachlorobenzene

pentachlorobenzene

hexachlorobenzene

\section{Chlorinated benzotrifluoride}

4-chlorobenzotrifluoride

3,4-dichlorobenzotrifluoride

2,4-dichlorobenzotrifluoride

trichlorobenzotrifluoride

tetrachlorobenzotrifluoride

\section{Chlorinated toluenes}

monochlorobenzylchloride*

dichlorobenzylchloride*

trichlorobenzylchloride ${ }^{*} \ldots \ldots \ldots \ldots \ldots \ldots . \ldots \ldots$

34-trichlorotoluene

\section{Other chlorinated aromatics}

p-chloronitrobenzene.

o-chloronitrobenzene.

3,4 dichloronitrobenzene.

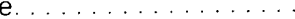

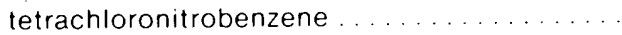

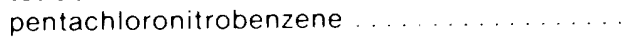

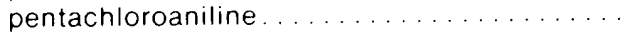

pentachlorophenyl methyl sulfide . . . . . . . . . .

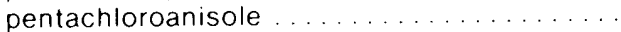

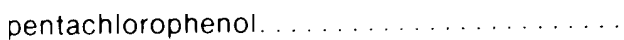

octachlorostyrene ..................

Chlorinated cyclics (non-aromatic)

$1,2,3,4,7,7$-hexachloronorbornadiene. . . . . .

1.2.3,4.5.7.7-heptachloronorbornene

5.6-epoxy-1,2.3,4,7,7-hexachloronorbornene. .
Zange in ppm

Locations

$$
\begin{gathered}
0.01-0.12 \\
0.001-0.17 \\
0.001-0.01 \\
0.1-0.3
\end{gathered}
$$

2.5

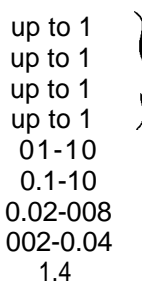

1.4

$\operatorname{tr}-67$

03-2.0

tr-0. 1

$0.2-0.8$

unknown

unknown

unknown

unknown

unknown

unknown

U.01-0.33

$0.01-0.33$

$0.01-0.33$

0.006-0.12

0.05

0.01

tr

0.03

0.26

0.08

tr. 0.15

0.1

tr 0.02

0.02-0.1

0.02-0.1

0.03-162

0.03-162

$0.03-16.2$
Delaware River. Del Buffalo River. N.Y.

Delaware River

Delaware River

Muskegon County. Mich. (water)

Muskegon County, Mich. (water)

Naukegan Haroor, III

jagınaw River. Micn. <ishacoquillas Creek, Pa. Jolumbia River. Wash.

Jolumbia River. Wash.

3UF-DO (animal fat)

I ombigbee Hiver. Ala

Jhio River. Onio

Viagara River, N.Y.

White Lake. Mich

Spring Creek. Pa.

Bald Eagle Creek. Pa.

Mississippi River. La

Kimmswick. Mo.

J.11 in peanut oll J.07 in peanut oil

Viagara Hiver, N.Y.

Niagara River. N Y

Kımmswick, Mo.

Cape Girardeau, Mo

St. Louis, Mo.

St. Louis, Mo.

Kimmswick. Mo.

Kimmswick, Mo.

peanut oil

peanut oil

peanut oil

peanut oil

many rivers and lakes

peanut oil

Mississippi River, La.

Lake Ontario. N.Y.

White Lake, Mich.

MississıppI Hiver, La. Mississippi River. La. Cape Girardeau, Mo. 
Table 17.-Chemical Contaminants in Foods ${ }^{\mathrm{a}}$-(cont.)

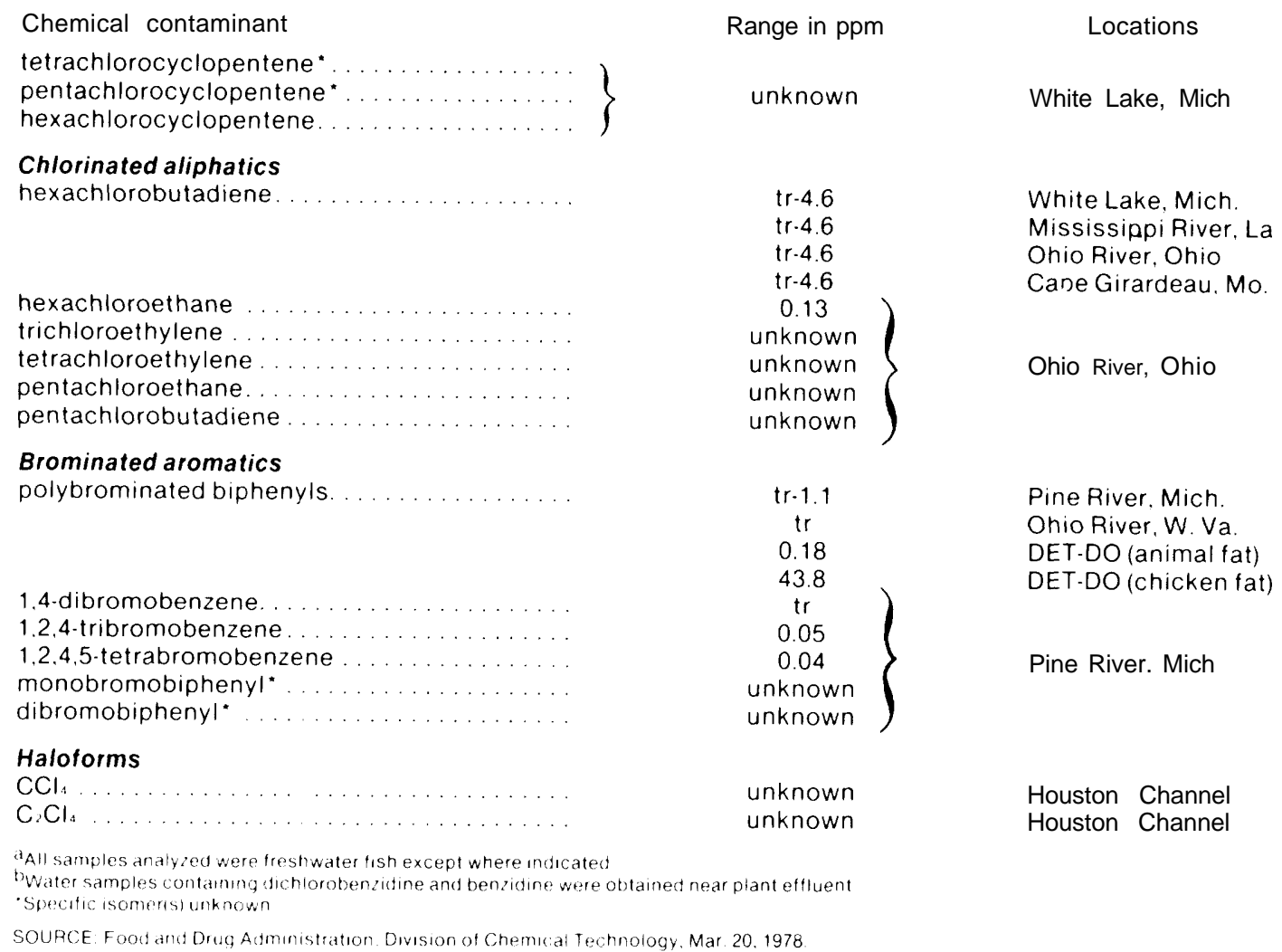

By applying appropriate criteria to the universe of industrial chemicals, it may be possible to detect potential environmental contaminants in food that have not been identified as significant by other methods. Although there is no truly independent way to verify the reliability $\mathrm{y}$ of priority lists, such lists could be generated for a pilot program designed to evaluate this approach vis-a-vis uncharacterized monitoring.

\section{Monitoring for Uncharacterized Environmental Contaminants}

Uncharacterized environmental contaminants are substances that may have entered the food supply but which have not been classified as regulated environmental contaminants or suspected environmental contaminants. Compounds may fall into this category because they are not known or suspected to occur in food. Because of a lack of toxicity data on compounds, they may not be recognized as threats to human health. This class of substances is similar to suspected environmental contaminants in that there are no stipulated analytical methods to detect them and no monitoring is mandated. Uncharacterized environmental contaminants are different from suspected contaminants in that none have been placed on lists of potentially harmful substances.

Although validated analytical methods for identifying uncharacterized environmental contaminants may be lacking, data on their presence or absence can be generated. Chemical analyses that are designed to show only the presence or absence of a compound in a sample are called qualitative analyses. In many cases an accepted analytical method for one class of compound will yield quantitative results for those compounds and qualitative results for others, Therefore, the pres- 
ence or absence of some suspected or uncharacterized environmental contaminants may be determined even though the chemist is not specifically looking for them.

In setting up a system of monitoring for uncharacterized environmental contaminants, some preliminary judgments would be made about the chemical nature of the target substances. Classes of compounds to be monitored would be selected on the basis of their structural characteristics, their use, and their suspected toxicity. Trace metals, halogenated hydrocarbons, or radioactive substances are examples of such classes. The class of compound determines the type of extraction required to separate the substance from other constituents of food, as well as the instrumentation needed to detect the presence of the substance.

The available analytical methods best suited for the class or classes of compounds under consideration would be selected. The question of whether analytical techniques are sufficiently advanced to support uncharacterized monitoring is explored in chapter VIII.

The establishment of an uncharacterized monitoring program would require the development of appropriate sampling and samplehandling guidelines. It would also be necessary to modify currently used analytical procedures. For example, a typical program for organic contaminants would involve preliminary screening to establish baseline levels of contamination in samples of food and water or selected indicator species over a given period of time. This information would then be used to develop an appropriate sampling plan to determine changes or trends over time. An increase in levels of an uncharacterized substance indicates its entry into food and water. This finding would trigger additional analytical efforts to characterize the new compound. Preliminary information on the substance's structure would be transmitted to toxicologists for evaluation and comparison with available information on known toxic compounds. If alarming trends or changes were observed, corrective regulatory actions could be taken.

\section{Specimen Banking}

It is difficult to detect environmental contaminants unless one is specifically looking for them, Monitoring methods for identifying suspected and uncharacterized environmental contaminants promise to partially alleviate the problems. Yet, even as new analytical instrumentation is developed and scientific knowledge expands, there will be new classes of compounds discovered in foods that have been present for years but were undetectable with then-existing instrumentation. Information on how long the compounds have been in food, what kinds of foods are affected, and from what areas the foods were derived would be of great help to epidemiologists and public health officials who must decide whether or not the chemicals have had (or will have) an adverse impact on the public.

One approach to this problem is the collection and storage of samples on a regular basis and in a manner that will protect their chemical integrity. In the future, when new instrumentation is developed or a toxic compound is discovered in foods, samples can be withdrawn from storage and analyzed. This in effect would be retrospective analysis. Investigators could go back in time, reconstruct events leading to a current situation, and estimate human exposures from the consumed foods.

There are examples of this kind of retrospective detective work. When high concentrations of mercury were discovered in tuna and swordfish, pollution was widely held responsible, But analysis of museum specimens that had been stored for decades indicated that the mercury levels were probably as high a century ago as now. Therefore, the mercury in fish may be from natural sources and may have always been so. This does not mean that the metal is not a potential health threat but rather that the potential exposure from eating the fish has not changed much over the years. 
There is a problem in utilizing most existing collections for retrospective chemical analysis. Since samples were not collected for use in chemical testing; they were not stored in a manner to maintain their chemical integrity. A 1975 survey of environmental specimen collections in the United States by Oak Ridge National Laboratories concluded that few of the existing collections were suitable for retrospective chemical analyses (2). Therefore, a need exists for a national program to collect, store, and maintain environmental samples (including food) to allow retrospective investigations,
EPA and the National Bureau of Standards are now working towards developing such a program by testing various methods of preserving samples for long periods of time without either adding unwanted chemicals or losing ones that are already in the sample. A number of scientists are encouraging this program and similar efforts (3-5). If continued funding is made available for specimen banking of environmental samples (including foods), future investigators will have an easier job of assessing what impact environmental contaminants in foods may have.

\section{SAMPLING}

Sampling involves the systematic collection of information from a portion of the environment. Sampling is done in such a way that the collected samples represent the whole in terms of the information desired. In regulatory monitoring the samples must be food commodities because the intent of the program is to determine the levels of regulated substances in the food supply. This information is the basis for enforcement actions.

Food samples may not be the best indicators if the monitoring is meant to serve as an early warning system-in other words, to detect a substance soon after it enters the environment and before it gets into foods. It may be better to analyze nonfood samples such as river sediments, water, or uneaten organs from food animals (the organs may concentrate the substance to analytically detectable levels before it can be seen in the flesh). The finding of an environmental contaminant in nonfood samples would trigger the examination of foods.

The following discussion outlines the primary considerations in selecting samples for investigatory monitoring systems. Constructing a sampling plan for such systems involves a number of decisions based on preliminary information about the nature and extent of environmental contamination. Such decisions include the number, sites, frequency, and types of samples.
The number of samples to be taken depends on how much risk of being wrong we are willing to accept. In other words, to what degree of certainty do we want to know that our food is free from environmental contaminants? One-hundred percent certainty would require analysis of every food item. Acceptance of a lesser degree of certainty allows the use of less costly sampling approaches.

Before preliminary data are collected, there is no way to calculate the exact number of samples needed to yield an answer of specified certainty. The most difficult factor to estimate is the variation specific to each contaminant and how it changes over time and space. This kind of information would have to be collected in pilot programs for suspected and uncharacterized monitoring before a national sampling plan could be developed. The number of samples taken will probably be constrained by the money, manpower, and available laboratory resources.

The density and location of sampling sites depend on the socially acceptable level of uncertainty, whether the contaminant stems from a point or nonpoint source, and how it is transported in the ecosystem.

If one is dealing with widely distributed nonpoint source environmental contaminants that are transported through water, the ideal sampling locations would be rivermouths that 
are discharge points of major watersheds. one could very effectively monitor the industrial portion of Michigan by sampling shellfish or fish from some two dozen major rivers just as they enter the Great Lakes. Baseline levels in these foods could be determined, but the origins of the contaminants would be difficult to determine (6).

The other extreme is to monitor food products on a production, site-specific basis. If the aim were to inventory all industries (including agriculture) that utilize and/or discharge a toxic substance, one could then routinely monitor food products, fish, and game at each identified site. Theoretically, the kepone and polybrominated biphenyls (PBB) situations would have been detected much earlier with such a system. The number of operations (large and small) that would need to be monitored is unknown, but the total appears to be so large that the costs would preclude consideration of this alternative (6).

A reasonable compromise approach is to use information generated under the Toxic Substances Control Act on the types of chemicals manufactured at various locations to guide in the development of a sampling plan for use in investigatory monitoring systems. The sampling plan would focus on some food organisms and some nonfood items. The data derived from analyses of such samples would yield the greatest information about environmental contamination trends in the region from which the samples were drawn.

The frequency of sampling depends on the rates a t which the contaminant moves through, accumulates in, and decomposes out of the food production system being monitored, Different food production systems have different genetic and environmental characteristics that determine the rates of material dynamics or transfer. For a beef feedlot, a range of 50 to 180 days would include the period involving a single-batch process. For an apple crop, a single sample per year would suffice. The frequency of sampling may be different for each type of production process. Once the species and the characteristics of the production systems have been identified, the appropriate sampling frequency can be determined (6),

The selection of the types of samples to be collected is also critical in identifying environmental contaminants as they enter the food chain. Although biological samples offer many advantages in monitoring systems, nonliving samples may be preferable in some instances. An example might be bottom sediments from rivers, lakes, or estuaries. Bottom sediments are derived, for the most part, from erosion of land and often bring with them to the aquatic environment substances that are used on land such as herbicides or pesticides. Moreover, once they are in the aqueous environment, they can "sorb" or concentrate many substances found in industrial discharges. The contaminated sediments then serve as a mechanism to expose the plants and animals that live in the waters to a particular chemical. Contaminated sediments may also be used to pinpoint the source of a chemical once it has entered the river, lake, or estuary (6).

Other types of nonliving samples might include air, river water, drinking water, or rain. All have certain advantages and disadvantages. For instance, the concentrations of many environmental contaminants in air and water are very low, causing problems for the analytical chemist. When a substance is found in air or water, it is sometimes difficult to determine where it entered the system. However, because we breathe the air and drink the water as well as eat the food from these environments, air and water cannot be eliminated as potential samples (6).

The most appropriate biological samples in an investigatory monitoring system should reflect key elements in the human food chain. Samples may include not only traditional agriculture products but also fish, game, shellfish, crustaceans, and wild fruits and nuts, Many of these wild foods also accumulate both point and nonpoint source environmental contaminants. Criteria for selection of 
the exact organisms should include the following characteristics:

- position in the food chain,

- lifespan,

- feeding behavior,

- understanding of the organism's physiology and biochemistry,

- body fat content,

- mobility,

- availability for sampling, and

- utility to humans (6).

Once a sampling plan is developed and samples collected and analyzed, the data must be presented in a form useful to the regulator. The data analysis should be rapid and provide information on trends as well as specific concentrations without significant distortion or deletion. Even with the bestdesigned computer retrieval system, the necessary data bank would become extremely large, complex, and expensive.
The supply of data must also be timely. If one wishes to regulate the level of an environmental contaminant in food when concentrations vary weekly, a monitoring system that reports data with a 6-month delay is not workable.

Finally, to develop information on exposure trends and determine the effectiveness of regulatory monitoring and enforcement, human tissues, blood, and urine can be analyzed for the presence of environmental contaminants. Human monitoring can be performed for either regulated, suspected, or uncharacterized substances. A sampling plan to detect trends in exposure among different population groups could be developed, but data generated from human monitoring would be unable in most cases to identify the source of exposure. At the present time, EPA houses the principal human monitoring program. This program is primarily concerned with pesticide residues.

\section{QUALITY ASSURANCE}

To ensure that data generated by any monitoring strategy are as accurate as possible, schemes have been developed to pinpoint errors. These schemes are called quality assurance programs. Such programs are mandatory in analytical laboratories because the possibility y of errors always exist.

Errors arise from a number of sources. For instance, impure chemical reagents, dirty glassware, or sample containers can impart contaminants to the sample that may interfere with the analysis and result in false readings. Instruments are not always stable and may give false readings. Some samples may contain substances that interfere with analyses, or a substance may be bound in a sample in such a way that normal extraction met hods will not extract it. Another factor is the potential for human error in the laboratory. These factors, singly or in combination, can lead to reported concentrations that are in error. Since commodities that violate standards could be marketed and consumed if the results were erroneously low, human health might be affected. If the results are erroneously high, undue economic hardship may be imposed on the food producer.

The Federal agencies that monitor foods for environmental contaminants are aware of these problems. Thus they use standardized analytical methods that have been tested and therefore offer some assurance that the results will be acceptable in court. When a violative sample is found, the product or batch is reanalyzed whenever possible to assure that results are valid.

These two practices are part of a quality assurance program. There are others as well. When a new chemical extraction or analysis technique is tested, it is important to analyze a sample $\mathrm{w}$ i $\mathrm{t} h$ known composition $\mathrm{t}$ o check the validity of the technique. Also, during routine determinations samples of known composition should be analyzed to check on the other types of potential errors. The samples of known composition are called "reference material" and may come from 
various sources including the National Bureau of Standards and EPA,

Available reference materials do not always satisfy the needs of current chemical monitoring programs, since such materials do not contain many known environmental contaminants. Moreover, the contaminants may not be stable under the storage method used. This is particularly true for synthetic organic chemicals. Another problem is that the type of reference material-i.e., beef liver-may not be similar enough to the food samples to be analyzed--i.e., fish-to be very helpful.

This points up an important gap in our ability to analyze accurately for environmental contaminants in foods. The variety of reference materials and the variety of compounds of known concentration in these materials are insufficient to satisfy the needs of the analysts. More effort must be expended to correct this problem.
Collaborative studies that involve more than one laboratory or group analyzing the same sample by the same or different methods are part of a quality assurance program. If all results are similar within acceptable limits there is some assurance that the method(s) are precise and perhaps accurate,

Often, more than one method can be used to measure a given contaminant. Confidence in accuracy can be increased if the methods agree. This is one of the reasons that a monitoring laboratory should have several methods available.

All of these aspects are important to assure that the proper answers are generated by a monitoring laboratory. All are timeconsuming and expensive. Therefore, any chemical monitoring program must allocate as much as $10 \mathrm{t}$ o 20 percent of its time for this increased workload.

\section{CHAPTER VII REFERENCES}

1. Clement Associates, Inc. Priority Setting of Toxic Substances for Guiding Monitoring Programs. O'TA Working Paper. 1979.

2. Becker. I). A., and F. J. Maienthal. Evaluation of the National Environmental Specimen Bank Survev. U.S. Environmental Protection Agency Report EPA-60011-77-015. Fnvironmental Health Fiffects Research Service. 1977.

3. "Proceedings of The Bi-State Conference on the Chesapeake Bay." Patuxent. Mo., The Chesapeake: Research Consortium, Inc., Annapolis. Md., CR(: Publication 61, 1977.

4. Goldberg. F. NOAA Workshop. Estes Park. colo. Julv 10)-14,1978.
5. International Workshop on Monitoring Environmental Materials and Specimen Banking, West Berlin, Oct. 23-28, 1978.

6. Comper. W. E.. and R. V. Farace. Toxic Substances in Food and Information Systems: Design and Management. O'TA Working Paper. 1979.

7. Food and Drug Administration. Study Group on FDA Residue Programs. FDA Monitoring Programs for Pesticide and Industrial Chemical Residues in Food. HEW Publication No. (FDA) 79-2116, 1979. 
Chapter VIII

Monitoring Instrumentation 


\section{Monitoring Instrumentation}

Various instruments have been designed to detect and quantify organic chemicals, trace metals, and radioactivity in foods. This chapter focuses on not only the technological state of this analytical art but also how such instrumentation could be applied in an investigatory monitoring program.

\section{ORGANIC ENVIRONMENTAL CONTAMINANTS}

\section{Analysis}

The vast number and wide diversity of natural and synthetic organic chemicals pose difficult analytical problems. Many methods and instruments have been developed to detect and quantify specific environmental contaminants in foods. There are generally three steps required for each type of analysis: extraction, cleanup, and detection and quantification. Figure 6 is a flow chart depicting how these steps would be applied to the analysis of the Environmental Protection Agency priority pollutants in food.

Extraction usually involves mixing the food sample with a selected solvent. In this step, the chemical is removed from the food and dissolved in the solvent. The time required for this step depends on the physical and chemical characteristics of the food sample and the substances. In some cases, the process may take 24 hours or more. The initial extract contains not only the substance of interest but possibly other organic compounds of similar volubility that must be removed before analysis.

The second step is known as the cleanup or isolation stage. The complexity and time required for this procedure depends on the food sample and the number of substances to be removed. In many cases multiple cleanup steps are necessary. The end products of cleanup procedures are fractions containing different classes of organic compounds.

The final step, detection and quantification, requires the use of highly sophisticated instruments and techniques. Among the most frequently used methods are mass spectrometry, gas chromatography, and liquid chromatography. Table 18 summarizes the techniques available for qualitative and quantitative organic analysis. More information can be found in appendix $G$.

The gas chromatography is an instrument designed to separate, identify, and quantify organic compounds. In simplified terms it consists of four components: an injection port, a column, a detector, and a recorder. There are many types of injection chambers, columns, and detectors, but the principles of operation are similar.

Gas chromatography involves vaporization of the sample to be analyzed. The gaseous sample then passes through a long tube or column packed with a solid matrix which is coated with an organic compound. This is called the "liquid phase." The rate at which various organic compounds in the sample pass through this column is a function of various chemical and physical properties, notably molecular weight. polarity, and geometric structure. The time of passage through the column is called the "retention time. Each compound has a characteristic retention time in the column.

To determine when and how much of each substance leaves the column, a detector is placed at the end of the column. The retention time can be used to identify each compound, while the strength of the electrical signal from the detector indicates the quantity. The 
Figure 6.- A General Scheme for the Qualitative and Quantitative Analysis of the Organic EPA Priority Pollutants in Semisolid Foods

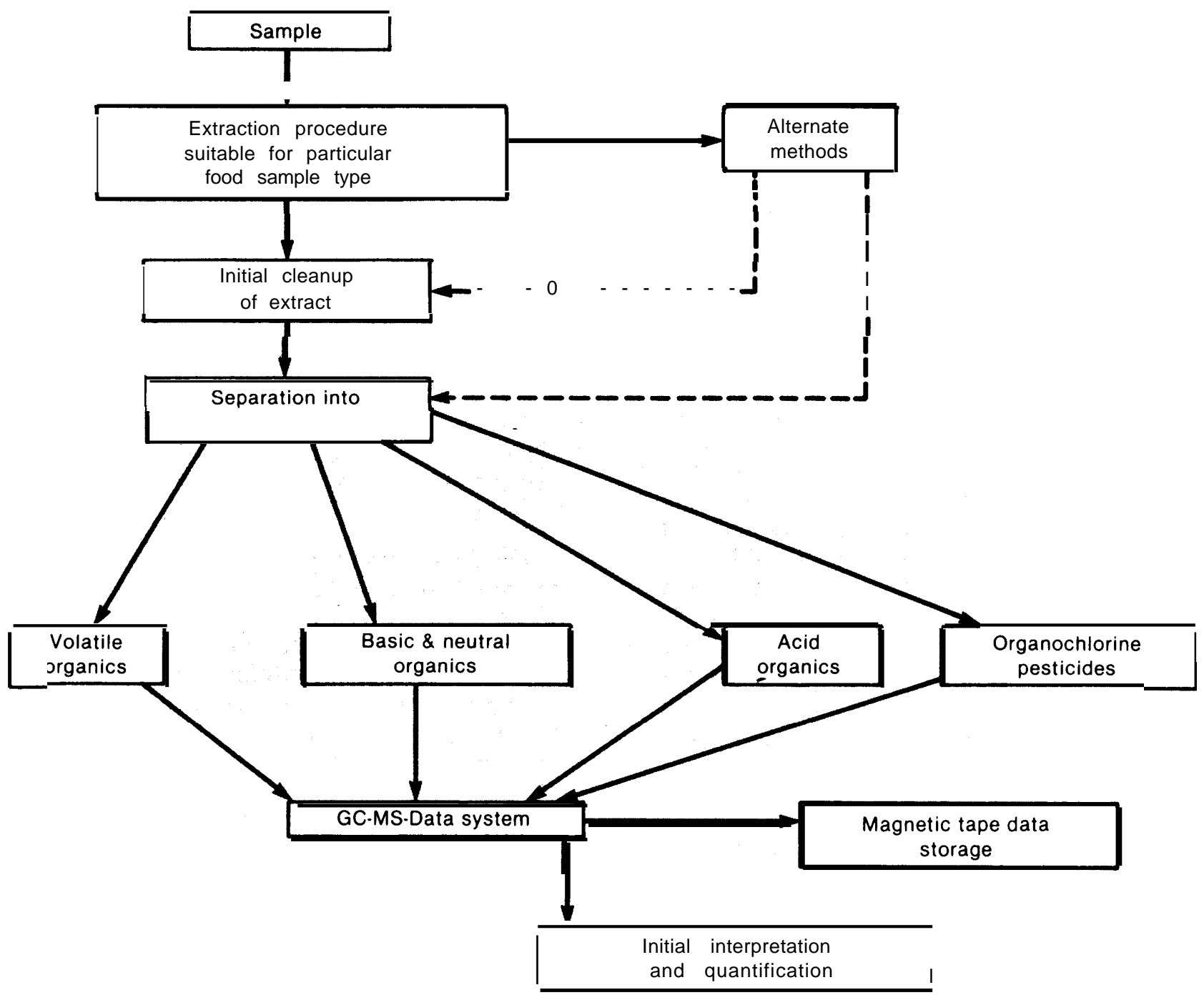

a Identification is based on the presence of characteristic ion fragments and associated chromatographic retention time data. Absolute identification would require a detailed interpretation of the complete mass spectrum of the organic compound of interest.

SOURCE John L Laseter Approaches to Monitoring Environmental Contaminants in Food OTA Working Paper 1978

identity of the compound is confirmed by comparing its retention time to that of a series of standard solutions (of known composition) injected into the gas chromatography under the same conditions. The areas under the graphed peaks of a sample readout or "fin- gerprint" are compared to a standard "fingerprint" to determine how much of each substance is present in the sample, If a peak in the sample fingerprint, for example, has the same retention time but twice the area as one in the standard fingerprint, it is assumed 
Table 18.-Techniques Available for Qualitative and Quantitative Organic Analysis

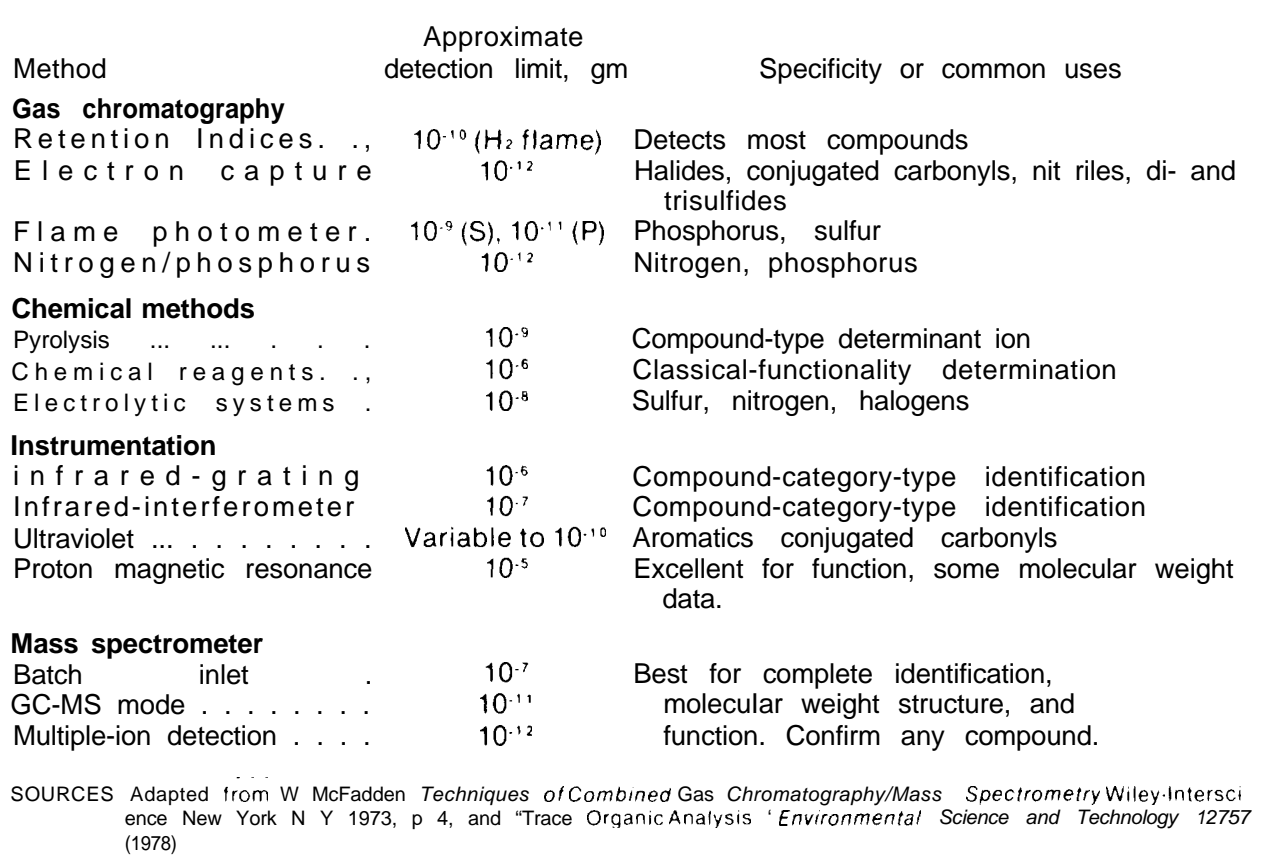

that the identity of the sample peak is the same as that of the known standard but that twice as much is present.

Recent developments in analytical organic chemistry promise new, more sensitive techniques for monitoring of synthetic organic chemicals in foods. These developments include high-resolution glass capillary columns for gas chromatography, and the linking of gas chromatography to mass spectrometers.

The glass capillary column is much more efficient in separating compounds than the older "packed" columns (figure 7). The gas chromatograph's packed column separates the individual components so that they exit the column and enter the detector one at a time. Some of the early data on the pesticide family DDT-DDE-DDD were incorrect because polychlorinated biphenyls (PCBs) had similar retention times in packed columns. As a result, reported concentrations of DDT, DDE, and DDD were often too high because the peak areas were really reflecting a combination of pesticides plus PCBs (l). Because of their superior resolution power, glass capillary columns avoid some of these problems.
Also because less cleanup is required, their use reduces the possibility that an important environmental contaminant may be removed in the process of preparing a sample for analysis.

Although the capillary column offers a number of advantages, it is more expensive and difficult to use than the standard packed column. More highly trained personnel are required to operate capillary columns and interpret results. However, many chemists feel that the advantages far outweigh any difficulties. Glass capillary columns are not now widely used for monitoring synthetic organics in foods. Packed columns continue to play an important role in the organic chemistry monitoring laboratory, but their future use may be restricted to more specific analyses.

Gas chromatography are currently the primary means by which laboratories monitor for organic chemicals. Some chemists, though, prefer mass spectrometers because of their high sensitivity and flexibility $(\mathrm{z})$. The coupling of a gas chromatography to a mass spectrometer introduces a new dimension that allows a chemist to identify compounds 
Figure 7. - Comparison of the Gas Chromatographic Analysis of a Standard Mixture of Polychlorinated Biphenyls (Aroclor 1254) on Two Types of Gas Chromatographic Columns

A: Analysis Using a Conventional Packed Column

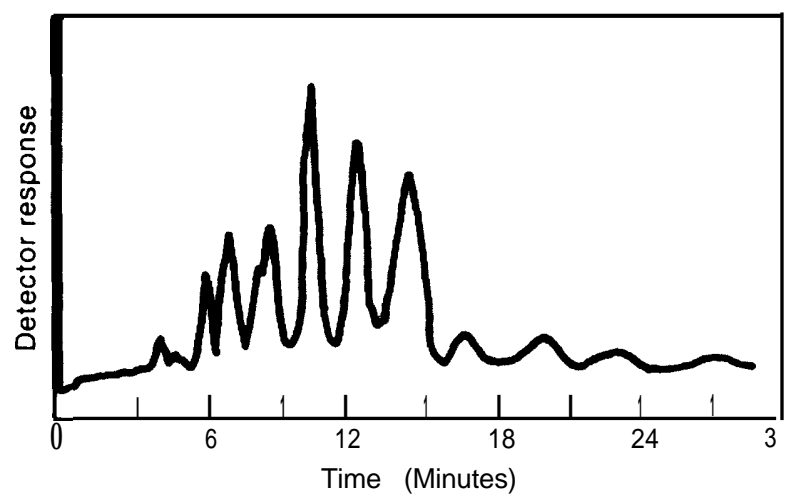

B: Analysis Using a High-Resolution Glass Capillary Column

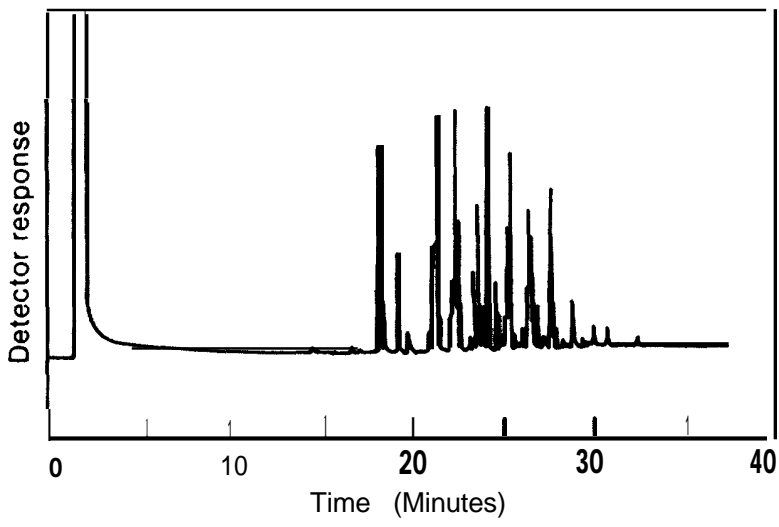

SOURCE John L Laset II Approaches to Monitoring Environmental Contami nants in Food OTA Working Paper 1978

classed as uncharacterized (3). Moreover, results from a mass spectrometer can confirm the identity of known chemicals whose presence (as indicated by gas chromatography alone) is in doubt because of sample contamination or other factors. Mass spectrometers are expensive and in addition require computerized data management systems.

Because of their physical and chemical characteristics. many organic compounds cannot be analyzed by gas chromatography.
Therefore, instruments such as infrared spectrophotometers and high-pressure liquid chromatography are necessary. These instruments add many thousands of dollars to the costs of setting up and equipping an up-todate organic chemical-monitoring laboratory.

\section{Application to Investigatory Monitoring}

Although the instrumentation described above has been available for several years, it has only recently been utilized for investigatory monitoring. Experimental projects incorporating computer analyses are now under study in at least three research facilities: the Bodega Marine Laboratory, the University of New Orleans, and the Virginia Institute of Marine Science, These projects employ highresolution glass capillary gas chromatographs coupled to mass spectrometers and sophisticated computer analyses, The resulting "fingerprints" or readouts from a sample extract are highly complex and show the presence of not only known or suspected environmental contaminants but also many organic compounds that must be classified as uncharacterized.

The three research groups are now testing an approach to determine which compounds deserve further testing. The gas chromatographic fingerprints are computerized so that information on retention times and peak intensities are stored. Subsequent samples are collected and analyzed and the data are computerized in the same manner. By comparing results from the same location over time (or with samples taken from other locations), one can determine whether peaks are increasing or decreasing, whether new peaks have appeared or old ones have disappeared, or whether some peaks occur only in some locations.

With appropriate computer programs or "soft ware," the data bank could be asked if there is a peak that occurs in samples from only one area or location. If so, the investigator can assume that the compound is anthropogenic, or man-induced, The compound could then be identified and its source con- 
trolled before the concentration increased to the point that a major contamination episode occurred. This type of monitoring could have detected kepone in the James River, Va., long before thousands of pounds of the pesticide entered the river.

Another question which could he asked of the data bank is whether there is a peak that is increasing with time. In other words, does sampling over time indicate that a compound is becoming more concentrated? Such a finding would flag the compound as one which merits attention, Had this type of monitoring program been in effect and this question been asked earlier, widespread pollutants such as PCBs could have been detected long before they were,

Other information from the chemical labora tory car-r draw attention to an uncharacterized substance deserving attention. For example, gas chromatographic detectors exist that respond mainly to organic compounds containing halogens such as chlorine or bromine. Because naturally occurring halogencontaining organic chemicals are rare and are far outnumbered by manmade ones, an uncharacterized peak from a halogen-specific detector may represent a manmade compound, Historically, halogen-containing organics such as DDT, PCBs, polybrominated biphenyls (PBBs), and kepone have caused major pollution crises. Therefore, such information may be sufficient to focus attention on a given peak from a halogen-specific detector.

Uncharacterized substances may or may not be dangerous to human health if consumed. But no assessment of toxicity can be made until the compounds are identified. To perform the chemical and physical tests necessary to identify even one uncharacterized peak may cost from $\$ 10,000$ to $\$ 100,000$, and in some cases even more.

\section{DETECTING AND QUANTIFYING TRACE METAL CONTAMINANTS}

Trace metals pose many of the same problems that plague the analyst monitoring for organic chemicals. However, the number of trace metals is much smaller. If only the total concentration of a metal is sought, more rigorous extraction procedures can be employed. But some metals, such as mercury, form organic complexes--for instance, methylmercury--which give rise to a subset called metallo-organics. These metallo-organics are not stable under harsh conditions and are often changed during rigorous extraction procedures.

\section{Analys is}

Analysis for trace metals usually requires extraction and cleanup before the quantitative analysis can be performed. Extraction often involves destruction of the sample's organic structure to release the metals from the solid or liquid food. There are a few analytical instruments such as X-ray emission spectrographs that can accept solid or liquid sam- ples, thus eliminating the extraction step (4). But these instruments cannot detect all metals at environmental concentrations.

Depending on what instrumentation is used in the final analysis, some cleanup of the extract may be required. The cleanup step is not nearly as frequent with trace metals, however, as with organic chemicals except if a metal lo-organic complex is sought. For metallo-organic compounds cleanup and separation procedures may be similar to those for organic compounds. After extraction and possibly cleanup, the sample is ready to be analyzed by an instrument to determine which metals, and how much, are present. There are a variety of instruments available for the determinations.

Probably the most commonly used instrument is the atomic absorption spectrophotometer (AA). The cost of this instrument is nominal $(\$ 15,000$ to $\$ 25,000)$, and $\mathrm{i} t$ can be operated by a well-trained, motivated technician 
with a high school chemistry and physics background working under the supervision of a chemist. One drawback of this instrument is that only one metal can be analyzed at a time.

In contrast, more sophisticated instrumentation such as X-ray emission, proton-induced X-ray emission, or plasma emission spectrometers can cost in excess of $\$ 100,000$. With these instruments, 20 to 60 elements can be determined simultaneously. These more sophisticated instruments require more highly trained personnel to operate and maintain. Table 19 summarizes the techniques available for qualitative and quantitative analysis of trace metals. More information can be found in appendix $\mathrm{H}$.

\section{Application to Investigatory Monitoring}

It is important to note that most AA units can detect only the specific element (metal) that they are set up to analyze. By contrast, the gas chromatography may sometimes detect the presence of organics other than those being sought. As a result, metals are more likely than synthetic organics to escape detection simply because they are not programed for analysis.

With the rapid development of lower cost techniques for detecting more than one element at a time, it would be possible to obtain data on trace metals in foods that were previously not sought and therefore not obtained.
This is analogous to uncharacterized organic monitoring where many unsought compounds can be found in the analysis of a food extract. Given the proper data systems, these "other" metals can be tracked, and perhaps pollution crises can be averted by detecting anomalous concentrations early enough.

\section{Speciation of Trace Metals}

Often the active or functioning forms of elements must be identified and measured. The need for the development of analytical methodology to identify and quantify the chemical form of metals found in the environmenti.e., chemical speciation-is pointed up by the following examples.

- Although arsenic is toxic, the plus three oxidation state, As (III), is more toxic than the plus five state, As (V). The compound arsine, $\mathrm{AsH}_{3}$, is perhaps the most toxic chemical form of arsenic.

- Alkyl (organic) mercury compounds pose greater propensities for bioaccumulation and the associated health effects than do the more common inorganic forms of mercury (but these also can vary greatly in toxicity).

Chemical forms or states are an important determinant of a trace metal's toxicity in biological systems. The selection of elemental analysis techniques capable of specifically measuring those chemical forms most important from a biological-effects viewpoint

\section{Table 19._Techniques Available for Qualitative and Quantitative Trace Metal Analysis}

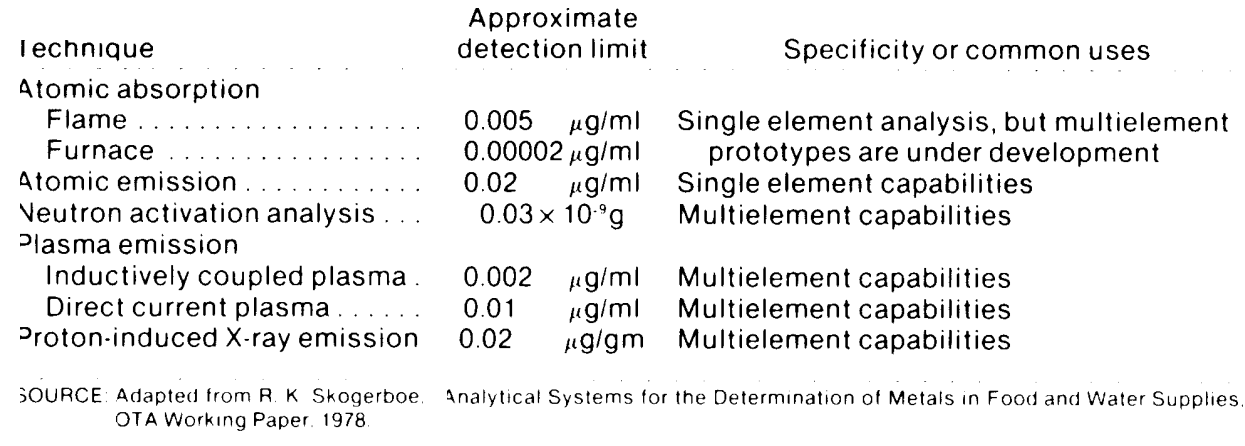


should be a primary objective of any environmental monitoring program.

The status of the present analytical technology is inadequate for this purpose in most routine monitoring programs. As a result, the functioning forms of most elements cannot be adequately studied. Thus the determination of the important (threshold) concentration levels for various elements typically has been rather crude. The most important current research need related to trace metal monitoring is the development of methods to measure the species rather than the total amount of trace metals.

\section{ANALYSIS OF FOODS FOR RADIOACTIVITY}

\section{Analysis}

Measuring radioactivity in foods is a physical process. It is most efficient when the radioactivity from a relatively large sample can be placed close to a detector. This means that direct measurements of radioactivity in bulk samples are only useful when levels of contamination are relatively high. Most measurements are preceded by preparation and possibly chemical separation to reduce the bulk of the material and to improve the efficiency of the measurement.

Foods generally have a high water content. The primary method of reducing bulk is freeze-drying or drying at room or at elevated temperatures. Most of the radionuclides of interest are not volatile under these conditions. Only elements such as tritium or iodine may experience losses. The dried materials can be reduced further by ashing at elevated temperatures, by cold-ashing with oxygen, or by wet-ashing with oxidizing acids. Dry-ashing is the simplest process, but it is most likely to lead to loss of volatile elements. With care even cesium, polonium, or lead can be retained. The other processes should not result in losses of elements of interest, except for iodine, tritium, and carbon.

Another way to reduce bulk is to treat either the original sample or the dried or ashed material with acids or other solvents and thus remove the desired elements from the bulk of the sample. This requires considerable testing beforehand to be certain that the process operates in the desired manner. The radionuclides of interest in ashed foods should be soluble in strong acids if ashing temperatures have not been excessive. If there is concern that insoluble particulate may be present, it is necessary to use more drastic methods such as fusion to bring the entire sample into solution.

In addition to bulk reduction, radiochemical separations are required to isolate the desired radionuclide both from the remaining bulk constituents and from other radionuclides that would interfere in the measurement. It is also necessary to convert the final product to a form suitable for presentation to the counter. This may involve electrodeposition, precipitation, or other processes.

The actual mass of radionuclide that is measured is almost always extremely small. As a result, many of the normal chemical reactions used in analytical chemistry to isolate an element are not appropriate. For instance, precipitates may not form. Therefore, it is common to add a few milligrams of a carrier material, preferably the inert form of the same element. When such an inert form does not exist, it is frequently possible to use similar elements as a carrier (for example, substituting barium for radium). The inert form then follows normal chemistry, carrying the radionuclide with it. It is also worth noting that even when the separation technique does not depend on the mass of element present, a carrier may still be useful in preventing unwanted coprecipitation or absorption on glassware.

In the chemical separations there is considerable use of classical analytical chemistry based on precipitation. In most cases, precipitation will be the final collection step in 
preparing the desired radionuclide for counting. In other cases the techniques of ion exchange, liquid extraction, distillation, and electrolysis may be required to prepare the sample for counting.

In certain cases, the total amount of a sample may be limited, and analysis for several radionuclides may be required. Procedures should be available for the sequential analysis of single samples, even though separate samples are used for routine work.

The measurement method used depends on the type of radiation, the form of the sample, and (to some extent) the amount of radioactivity. The selected analytical procedure should be designed so that the sample is brought to a suitable form for the equipment and conditions that exist.

The major emitted radiations are generally grouped as alpha, beta, and gamma. Alpha radiation is characteristic of the natural and artificial radionuclides of high atomic weight and consists of energetic particles with very low penetrating power. Its hazard is significant only within the body, where alpha-emitting nuclides can irradiate specific sensitive tissues. Beta radiation appears in both natural and manmade radionuclides, and consists of electrons possessing kinetic energy and modest penetrating power. Gamma radiation is pure electromagnetic radiation and is extremely penetrating. Thus, it is hazardous externally as well as when it is present in the body. Gamma radiation can be directly measured in foodstuffs, while alpha and beta emitters generally must be separated from the bulk constituents of the sample before they can be measured.

It is possible to measure the total gamma, total beta, or even the total alpha activity in a sample of food. Unfortunately, such data are valueless in estimating human exposure. The accuracy of such estimates is very poor because natural potassium usually interferes, and the chemical and radiation characteristics needed to evaluate possible hazards are not known.
It is possible, however, to set a particular total activity level as a screening level for a specific food, If the measured value is below the screening level, no analyses for individual radionuclides are performed. In such a case, the measurements should be considered internal data and the numerical results should not be published, Any report should merely list the samples as having activities below the stated screening level. Table 20 summarizes the typical limits of detection for radionuclide measurement.

Alpha Emitters.-The measurement of alpha activity is best carried out on a very thin sample to avoid self-absorption of the alpha particles. Most metals are electro-deposited onto small smooth discs of stainless steel, nickel, or platinum. Evaporation of pure solutions is used in some cases. The measurement of the total alpha activity can be conducted either in thin-window counters or by scintillation counting with zinc sulfide phosphor. Both techniques are highly efficient, but the scintillation method can give a considerably lower background with a consequently lower limit of detection.

A somewhat less-sensitive technique is the liquid scintillation spectrometer described in the next section.

Alpha spectroscopy provides two other alternatives: the Frisch grid ionization chamber or the silicon diode solid-state detector. The Frisch grid unit can handle large area samples but is slightly poorer in resolving closely separated energies. On the other hand, the silicon diodes available are all quite small and can only count samples of about $1-\mathrm{cm}$ diameter with high efficiency.

Beta Emitters.-Beta counting is a little more flexible in the mass of material that can be present at the time of counting. This is true for higher energy beta emitters but carbon-14 and tritium present a problem. Since each individual beta emitter gives off particles with a range of energies from zero to a characteristic maximum, beta spectrometry is not possible for food samples. 
Table 20.-Typical Limits of Detection for Radionuclide Measurement

\begin{tabular}{|c|c|c|c|c|c|c|c|c|}
\hline \multirow[b]{2}{*}{ Nuclide } & \multirow{2}{*}{$\begin{array}{c}\text { Counter } \\
\text { efficiency \% }\end{array}$} & \multirow{2}{*}{$\begin{array}{c}\text { Counter } \\
\text { background cpm }\end{array}$} & \multirow{2}{*}{$\begin{array}{l}\text { Chemical } \\
\text { yield \% }\end{array}$} & \multicolumn{5}{|c|}{ LLD (dpm) per sample for various counting times } \\
\hline & & & & $10 \min$ & $40 \mathrm{~min}$ & $400 \mathrm{~min}$ & $1,000 \mathrm{~min}$ & \\
\hline Amerlclum.241 & 25 & 0.001 & 60 & 03 & 015 & 0.05 & 003 & $\begin{array}{l}\text { Chemistry, } \alpha \\
\text { spectrometry }\end{array}$ \\
\hline Ceslum-144 & 22 & 04 & 75 & 6 & 3 & 09 & 06 & $\begin{array}{l}\text { Chemistry, } 3 \\
\text { counting }\end{array}$ \\
\hline \multirow[t]{2}{*}{ Cesium-137 } & 30 & 04 & 75 & 4 & 2 & 06 & 04 & $\begin{array}{l}\text { Chemistry, } \beta \\
\text { counting }\end{array}$ \\
\hline & 2 & 006 & 75 & 30 & 14 & 45 & 3 & $\begin{array}{l}\text { Chemistry, } \\
\text { Spectrometry (Ge) }\end{array}$ \\
\hline \multicolumn{9}{|l|}{$\begin{array}{l}\text { Cobalt- } 60 \text {, other } \\
\text { activated }\end{array}$} \\
\hline products & 1 & 005 & - & 100 & 50 & 15 & 10 & $\gamma$ Spectrometry $(\mathrm{Ge})$ \\
\hline Tritium & 30 & 5 & 75 & 15 & 6 & 2 & 1.5 & $\begin{array}{l}\text { BScintillation, can be } \\
\text { enriched electrolyt. } \\
\text { ically by } 10-25 \text { times }\end{array}$ \\
\hline \multirow[t]{2}{*}{ lodine- 131} & 25 & 0.4 & 80 & 5 & 2 & 0.7 & 0.5 & $\begin{array}{l}\text { Chemistry, } 8 \\
\text { counting }\end{array}$ \\
\hline & 4 & 03 & 80 & 25 & 13 & 4 & 25 & $\begin{array}{l}\text { Chemistry, } \\
\text { spectrometry(Nal) }\end{array}$ \\
\hline Phosphorus-32 & 45 & 03 & 60 & 3 & 1.5 & 0.5 & 0.3 & $\begin{array}{l}\text { Chemistry. i } \\
\text { counting }\end{array}$ \\
\hline Plutonium-239. -240 & $40 \quad 25$ & 0005 & 85 & 06 & 0.3 & 0.09 & 0.06 & $\begin{array}{l}\text { Chemistry, } \alpha \\
\text { spectrometry }\end{array}$ \\
\hline \multirow[t]{2}{*}{ Radium-226 } & 56 & 02 & 90 & 1 & 0.6 & 0.2 & 0.1 & $\begin{array}{l}\text { Chemistry, } \\
\text { emanation, }{ }^{222} \mathrm{Rn} \\
\text { counting }\end{array}$ \\
\hline & 40 & 0.001 & 85 & 1 & 0.5 & 0.15 & 0.1 & $\begin{array}{l}\text { Chemistry, } \alpha \\
\text { counting }\end{array}$ \\
\hline Strontium-90 & 45 & 0.3 & 80 & 2 & 1 & 0,4 & 0.2 & $\begin{array}{l}\text { Chemistry, is } \\
\text { counting }\end{array}$ \\
\hline Thor! urn-230, -232 & 3225 & 0001 & 70 & 0.3 & 0.1 & 0.04 & 0.03 & $\begin{array}{l}\text { Chemistry, a } \\
\text { spectrometry }\end{array}$ \\
\hline U-Isotopic & 25 & 0.005 & 75 & 0.7 & 0.3 & 0.1 & 0.07 & $\begin{array}{l}\text { Chemistry, } \alpha \\
\text { spectrometry }\end{array}$ \\
\hline
\end{tabular}

SOURCE N H Harley Analysts of Foods for Radioactivity OTA Work ing Paper 1979

The available counting equipment for quantitative measurement includes geiger counters, proportional counters, and scintillation counters. The geiger counter is relatively inexpensive and requires only simple electronics but is not popular and is generally not available as a counting system. The thinwindow proportional counter is used widely and has both reasonably high efficiency and low background. For low-level samples the background can be further reduced by anticoincidence techniques. These add to the complexity and cost of the system but are sometimes necessary.

Scintillation counting can be performed in two ways. Solid scintillators can be used for counting chemical precipitates collected on filter papers, and liquid scintillators can be used whenever the sample can be made miscible with the scintillating solution itself. This can even be done with solids by suspending them in a scintillating gel. The advantage of liquid scintillators is their high efficiency, even for the low-energy emitters carbon-14 and tritium. Scintillation systems for counting precipitates are not commercially available at present. There are, however, many liquid scintillation systems on the market, most of them with automatic sample changers. These use high levels of activity and short counting times. The better systems also have a provision for rather crude spectrometry. They can distinguish qualitatively and quantitatively among carbon-14, tritium, alpha emitters, and higher energy beta emitters.

Gamma Emitters.-Gamma rays are so penetrating that the detector must have a 
considerable mass to absorb enough energy to produce a response. Since energy absorption is required for spectrometry, solid detectors are most useful, Sodium iodide is a popular detector because crystals can be fabricated in large sizes and are transparent to the scintillations produced by radiation. Sodium iodide has high efficiency but poor energy resolution and is now applied to samples where some separation has taken place. The advantages are that samples of food can often be counted directly without chemical preparation. Milk is a good natural example, as the metabolism of the cow removes most gamma emitters other than isotopes of cesium, iodine, and naturally radioactive potassium.

More complex spectra can be resolved with the solid-state germanium diode detector. Interferences from radionuclide impurities are greatly reduced compared to spectra from sodium iodide detectors. The efficiency of the diode is low and, for many analyses, a spectrometer can only be used for one measurement a day, Another disadvantage is that the detector must be kept at liquid nitrogen temperature to maintain its detection capability.

Diode spectrometers may also be used to measure the low-energy gamma-rays that accompany alpha emission. This allows direct measurement in some environmental samples, but the levels in foods have not been high enough for this technique.

General Requirements.-The choice of a counting procedure depends on the precision required. The relative precision of a quantitative counting measurement, in turn, is inversely proportional to the square root of the number of counts obtained. Thus any improvement in precision must be obtained by increasing the number of counts. This can be done by using larger samples, by counting for longer times, or by using counters with higher efficiency, A secondary improvement is possible for low-activity samples by decreasing the background, Each of these improvements has some drawback, and selection of the optimum balance requires a weighing of cost, manpower, and quality.

\section{Applications to Investigatory Monitoring}

The monitoring of foods for radioactivity should not be considered a primary defense against human exposure. The first indication of hazards should always come from information on releases or from measurements of radioactivity in air or water. Once the existence of contamination has been established, foods can be analyzed to evaluate the potential hazard to man.

Knowledge of the source of radioactive contamination gives a good indication of the nuclides that can be expected in the sample. This information helps in planning the analysis, since requesting a complete analysis for all radionuclides or even for all types of radioactivity in a single sample would lead to a lengthy and expensive operation. Indeed, monitoring for suspected contaminants is more applicable than the uncharacterized monitoring.

The general groups of nuclides that maybe encountered include those that occur naturally such as radioactive potassium and members of the uranium and thorium series, artificial fission products such as transuranic elements, and other activation products that result from nuclear weapon explosions and nuclear reactor operations.

Fission products are a very complex mixture when they are formed, but the shortlived radionuclides die out rapidly and the mixture becomes simpler within a few days. The transuranics (plutonium, americium, etc. formed by activation of the basic fissionable material) are of some interest because of their high toxicity when incorporated into the body. Present evidence, however, indicates that their uptake through the gut is relatively small and that dietary intake is not a significant problem. The other activation products are frequently elements that make up steel or other metal containers or structural ele- 
ments. Radioactive manganese, chromium, cobalt, zinc, and iron are particularly common and result from interactions of the materials with neutrons released in the nuclear reaction, Contamination of foodstuffs with single nuclides is extremely unlikely, and more than one member of any group will probably be present in any sample.

In contrast to most other pollutants, the effects of radiation are considered to have a linear response regardless of the level, Thus there is no threshold and no absolutely safe limit. The analytical significance of this is that the lower limits of detection for radioactive substances have been brought down to very low levels. The simple yes-or-no testing for acceptability that satisfies regulators for many other pollutants in foods cannot be used.

\section{ESTIMATED COST TO EQUIP A LABORATORY T• CONDUCT INVESTIGATORY MONITORING}

Table 21 illustrates estimated costs, required space, and estimated downtime for a laboratory designed to conduct investigatory monitoring. In addition, the number of samples the system would be able to analyze in a year are estimated.

These figures do not reflect the total costs for establishing a national system of investigatory monitoring. Such a cost estimate would require information on the total num-
Almost all radionuclides of interest in cases of contaminating events now exist in foods in small but measurable quantities. Short-lived nuclides are the exception and the transuranic elements are only present at levels that require considerable effort in analysis. Since most of the radionuclides are already present in foods, measurements made for background information should produce a numerical answer, not merely an indication that the amount is less than some pre-set value. This accumulation of background data provides a valuable baseline for evaluating hazardous levels following a contaminating event. The natural activity data are equally valuable, since the amount of information on food concentrations is presently insufficient for valid comparisons with manmade radioactivity. ber of samples to be collected and analyzed. Information of this sort could be obtained through a pilot project to investigate the feasibility of the two investigatory monitoring approaches. This would determine the number of laboratories, the number of people and their salaries, and costs of equipment maintenance, supplies, and training. Once the system is setup and running, some savings might be realized through automation. 
Table 21 .-Estimated Costs to Equip a Laboratory to Conduct Uncharacterized Monitoring

Analytical instrumentation

\section{Synthetic organics}

1 . Small, high throughput, GC-MSdata system with automated liquid Injection device.

2. Electron impact/chemical Ionlzatlon-equipped highresolution mass spectrometer data system with automated

i $n$ je c t i o $n$ d e vice

3. Gas chromotographic flame

ionization detector, electron capture detectors (additional chromotographic systems may be $r$ e $q$ u i $r$ e $d$ ).

4 Liquid chromotograph interfaced to mass spectrometer system

5. Central data management system

6. Cold storage and processing facilities

Synthetic organics subtotal

Trace metals

1. Inductively coupled plasma

Multielement atomic emission

$\begin{array}{llllll}s & y & s & t & e & m\end{array}$

2. Flame and furnace atomic absorption spectrophotometer ( $s$ ingle element mode)

3. Electrochemical instrumentation

4. Central data management system.

Trace metals subtotal.

\section{Radionuclides}

1. Four position alpha spectrometer and detectors, multichannel analyzer and output

2. Germanium diode gamma spectrometer wit $\mathrm{h}$ detector, shield, electronics, PDP-11

computer and output

3. Four general purpose proportional c o u n t e r s,

4. Liquid scintillation spectrometer, automatic sample. or alphacounting capabilities

Radionuclides subtotal

Total. . . . . . . . . . . . . . . . . . . Approximate
capital cost

$\$ 100,000$

$\$ 200,000$ -

$\$ 300.000$

$\$ 15,000$

$\$ 75,000$

$\$ 1,000,000$

$\$ 500$ ! 000

$\$ 1,585,000$ -

$\$ 1,935,000$

$\$ 100,000$

$\$ 25.000$

$\$ 25,000$

$\$ 50.000$ -

$\$ 75.000$

$\$ 200.000$.

$\$ 225,000$

$\$ 21,000$

$\$ 100,000$ laboratories equipped with supporting

facilities and equipment are required

Approximately 2,500 ft.' with supporting facilities and equipment are required for items 1-4
Approximately $1,500 \mathrm{ft}$. '

Estimated

down

time

20- $50 \%$

uncharacterized environmental contaminants as well as known environmental contaminants

20- $50 \%$

$20-50 \%$

$20-50 \%$

20- $50 \%$

$3,000-5,000$

$3-15 \%$

SOURCES Adapted from J L Laseter Approaches to Monitoring Environmental Contaminants in Food OTA working Paper 1978 R K Skogerboe Analytical Sys terns for the Determination of Metals in Food and Water Supplies OTA Working Paper 1978 and N H Harley Analysis of Foods for Radioactivity OTA Working Paper 1979 


\section{CHAPTER VIII REFERENCES}

1. Butler, P. A. Environmental Protection Agency. Gulf Breeze Environmental Research Laboratory, personal communication, 1978.

2. Anonvmous. "Trace Organic Analysis," Environmental Science and Technology, $12(7)$ : 757, 1978.

3. Laseter, J. L. "Approaches to Monitoring En- vironmental Contaminants in Food, OTA Working paper, 1978.

4. Skogerboe, R. K. "Analytical System for the Determination of Metals in Food and Water Supplies, " OTA Working Paper, 1978.

5. Harley, N. H. "Analysis of Foods for Radioactivity," OTA Working Paper. 1979. 
Chapter IX

\section{Congressional Options}




\section{Congressional Options}

The present system of controlling environmental contaminants in food consists of two parts: regulatory procedures to set and enforce limits for environmental contaminants, and monitoring procedures to detect lots of food in violation of established limits. Each State has authority for regulating food grown and consumed within its boundaries. The Federal Government is responsible for regulating food in interstate commerce.

Congress can choose to maintain this system. But if it wishes to put greater emphasis on protecting consumers from contaminated food, one or more of the options discussed below could be adopted. None of these options (except for the first) are mutually exclusive.

\section{OPTION I-MAINTAIN THE PRESENT SYSTEM}

The current regulatory approach to controlling environmental contaminants in food involves the setting of action levels (and occasionally tolerances), coupled with regulatory monitoring for known [and a few suspected) contaminants. Food containing an amount of a contaminant that exceeds the action level or tolerance can be identified through such monitoring. This food is then removed from the marketplace. Public exposure is thus theoretically limited to those foods containing quantities of contaminants that fall under prescribed action levels or tolerances.

Pros: There are two principal advantages to maintaining this system. No additional appropriations or legislation are required. No changes in existing regulations are necessary.

Cons: There are a number of disadvantages in retaining the current system. The time needed to identify an environmental contaminant in food and take corrective action would not be shortened, The people would continue to be exposed until a contaminant was detected and identified, and an action level put into effect,

Moreover, a c t ion levels and tolerances tend to institutionalize rather than protect people against exposure, In other words, action levels and tolerances permit a certain level of contaminant to be present in food. Unless action levels or tolerances are reduced, little effort will be made to eliminate the contaminant, The threshold concept on which action levels and tolerances are based-that there are exposure levels to toxic substances below which there are no effects on health -is being increasingly challenged (especially when carcinogens are involved).

If high action levels or tolerances are established, exposure is not reduced. Lowered action levels and tolerances may reduce public exposure. But even low limits are set on the basis (among other things) of nationwide per capita consumption of a particular food. Contamination problems, however, may be localized and further influenced by regional food consumption patterns. Thus, a local population may be highly exposed to a toxic substance although the tolerance, based on national consumption, may be low.

Moreover, there is no requirement for review once an action level has been established. An agency is under no pressure to actively seek out new data that might alter a prescribed level, 
Tolerances and action levels have other weaknesses. They are often not easily applied when the environmental contaminant involved is a suspected carcinogen (such as polychlorinated biphenyls (PCBs)). Furthermore, the time required to perform a complete chemical analysis for contaminants in nonprocessed foods such as fish makes its dif- ficult to prevent some shipments from reaching the marketplace.

Finally, there are procedural problems in the present system. States have no clearly defined authority to which they can turn when they suspect environmental contamination of food.

\section{OPTION 2-AMEND THE FOOD, DRUG, AND COSMETIC ACT}

The Food, Drug, and Cosmetic Act has been amended several times since its passage in 1938 to deal with new food regulatory problems. Environmental contamination of food is now a national problem which Congress has never directly addressed through legislation. Thus, Congress could choose to give regulatory agencies more guidance by clarifying its position on environmental contaminants in food.

An amendment to the Food, Drug, and Cosmetic Act could include one or all of the following points. None are mutually exclusive.

\section{Option 2A-Simplify Administrative Procedures}

Under current law and regulations, the Food and Drug Administration (FDA) sets an action level for a contaminant soon after an interstate shipment of contaminated food is discovered. FDA will then presumably launch the elaborate rulemaking proceedings that culminate in the establishment of a tolerance (under section 701(e) of the Food, Drug, and Cosmetic Act). In reality, the costs and delays involved in the complex rulemaking procedures now required for the adoption of tolerances have discouraged FDA from moving from the first (action level) to the second (tolerance) state of the process.

Congress could amend the Food, Drug, and Cosmetic Act to simplify the administrative procedure through which tolerances are set. The changes could be modeled after section 553 of the Administrative Procedures Act. This process involves publication of a toler- ance proposal in the Federal Register, along with (as required by recent court rulings) the rationale and factual data underlying the proposal. The public can then respond to the proposal with written comments. FDA may also hold legislative-style public hearings to allow presentation of oral arguments and evidence.

After considering all of the comments, FDA publishes a final rule (in this case, a tolerance). This final rule includes explanation of any changes from the original proposal and responses to factual points raised by the public. Most agencies now use this model for rulemaking. It is a process that can be carried out expeditiously with modest investment of an agency's resources.

Pros: Adoption of this streamlined rulemaking procedure would reduce the time and expense now involved in setting a formal tolerance. It may encourage FDA to move from action levels to tolerances, thus bringing more public participation into the process.

Cons: Because action levels are administrative guidelines, they can easily be changed when new scientific information becomes available. Even if FDA comes to use a simplified procedure to set tolerances, it may still be slow to revise them in the light of new data.

$$
\begin{aligned}
& \text { Option 2B--Require the } \\
& \text { Establishment of Tolerances }
\end{aligned}
$$

Congress could amend the Food, Drug, and Cosmetic Act to require the establishment of 
a tolerance within a specific time after the setting of an action level.

Pros: This change would encourage FDA to gather additional information on a contaminant's toxicity and the public's exposure. It would speed up a process. that now operates under no deadlines. And it would result in definitive tolerances that FDA could enforce with less concern about judicial questioning.

Cons: This option, however, would substantially increase FDA's workload unless tolerance-setting procedures were simplified. Indeed, it has been the costs and delays of the current rulemaking process that have deterred FDA from moving from action levels to tolerances. Thus, tolerances should not be required without also simplifying present rulemaking procedures,

\section{Option 2C--Clarify the Role of Economic Criteria}

Congress could amend the Food, Drug, and Cosmetic Act to clarify to what extent economic criteria can be used in setting tolerances. The Act does not specify that costs (the adverse economic effects) of a proposed tolerance be considered when setting a tolerance. FDA, in practice, does weigh the cost of food lost when establishing a tolerance.

The Food, Drug, and Cosmetic Act could be amended to prohibit FDA from considering costs when setting a tolerance. Prohibiting any economic assessment would ensure that public health would be the first priority in setting a tolerance.

Conversely, the Act could be amended to require $\mathrm{FDA}$ to weigh the costs against the benefits of a proposed tolerance. Requiring FDA to evaluate the economic consequences of a tolerance would give FDA clear authority to weigh such estimated effects together with the potential health risks when establishing a tolerance. Congress could require FDA to gauge only the primary costs (as is now done with food lost) or all associated costs (food lost, employment impacts, distributional and indirect effects) for a proposed tolerance. The techniques available for estimating costs require up to a year for generating the necessary data. Thus, weighing the costs is best suited for setting tolerances. not action levels.

The advantage in including costs in tolerance-setting decisions is that adverse economic impacts are likely to be reduced. The disadvantage is that tolerance levels are likely to be higher than would be the case if costs were not considered.

Pros: By clearly defining to what extent costs can enter into tolerance-setting decisions for environmental contaminants in food, Congress would eliminate ambiguities of interpretation and provide clear guidance to FDA.

Cons: FDA has interpreted the Food, Drug, and Cosmetic Act as allowing cost considerations, Legislation requiring economic assessment could limit FDA's discretion to weigh the costs of food lost if it judges the situation warrants such a treatment.

\section{Option 2D--Establish Regional Tolerances}

This option would give FDA the flexibility to set different action levels or tolerances for different regions, based on expected levels of exposure, regional levels of contamination, and eating patterns.

Pros: Action levels and tolerances may not be set low enough to protect those populations that are most highly exposed, previously exposed, or most vulnerable. States may not exercise their authority to set tolerances which are more restrictive than the Federal tolerance because of budget limitations, inadequate information, or political pressures. FDA can provide guidance to States and suggest more restrictive tolerances or warnings to the public, but FDA has no authority to intervene if the contaminated food does not enter into interstate commerce.

Cons: Regional tolerances would complicate monitoring and enforcement programs. Regional tolerances might also be viewed as Federal infringement on State authority. 


\section{OPTION 3-ESTABLISH AN INVESTIGATORY MONITORING SYSTEM}

Environmental contaminants could be detected earlier in the food chain by improving present environmental monitoring capabilities - establishing an investigatory monitoring system while maintaining current regulatory monitoring programs.

Congress could set up a national investigatory monitoring system that monitors for either suspected or uncharacterized environmental contaminants. A system combining elements of both approaches could also be established. Since any of these monitoring approaches would require some research and development before a fully operational system could be devised, Congress could choose to create a pilot program. Such a program would spur research and development and assess the feasibility and cost-effectiveness of the various approaches.

The investigatory monitoring systems discussed in this assessment would call for different sampling and quality control procedures. The development of these procedures is as important as the development of monitoring technology (some of it still in the experimental stage). Indeed, there is no comprehensive investigatory monitoring system for toxic substances in food and the environment at any level of government.

Consequently, Congress might opt for a pilot project to assess the capabilities and resource requirements of various national monitoring systems instead of mandating a particular monitoring approach. Such a pilot project would focus on the monitoring of organic chemicals, inorganic, and radioactive substances. It would determine the technology, sampling, and quality control needs for monitoring these three toxic substance categories in food, air, water, and soil. The broader purpose of the project would be to develop a comprehensive monitoring program that would meet the Government's regulatory needs, provide data to make cost-effectiveness assessments of the alternative strate- gies, and reduce public exposures to environmental contaminants as much as possible.

\section{Option 3A-Establish a National Monitoring System for Suspected Environmental Contaminants}

Suspected environmental contaminants are substances that are most likely to enter the food supply and pose potential health hazards, Lists of such substances could be drawn up for organic chemicals, trace metals, and radioactive substances in order that they be monitored in the food, Various criteria such as toxicity, volume of production, occurrence in the environment, persistence, and biodegradability could be used in putting together the lists.

Pros: Present food monitoring efforts are not designed to detect new environmental contaminants in food. The limited amount of monitoring for suspected contaminants that does exist is primarily concerned with trace metals. But far more of this type of monitoring is needed to anticipate new contaminants in food.

Cons: To draw up such lists successfully, considerable information is needed. Substances for which there is little or no data would automatically be given low priority. In large part, the makeup of the lists would depend on scientific judgments. However, scientific judgments often vary (or even conflict). Thus, the reliability of the lists may be in question,

Priority lists of trace metals and radioactive substances, which are limited in number and already well-investigated, would be more reliable than a list of organic compounds. There are thousands of such organic agents manufactured, And there is little toxicological or environmental information available on a great many of them.

If such lists were developed, the number of substances monitored would necessarily be 
limited. Standards would be set up for determining which substances would get priority. Of course, there would be no certainty that unlisted substances might not get into the food supply and threaten human health. The cost of such a monitoring system would depend on how many substances were being traced as well as the expenses of equipping and staffing laboratories.

\section{Option 3B--Establish a National Monitoring System for Uncharacterized Environmental Contaminants}

Uncharacterized monitoring would be designed to detect substances that are: lacking toxicity data for potential human risk, not known to be present in food, and not even known to exist in the environment. This kind of monitoring is most needed for synthetic organic chemicals. The purpose is to detect changes in the levels of various synthetic organics in environmental or food samples over time,

Scientists would not necessarily know the identity of individual substances they were monitoring. But if the concentration of a particular compound substantially increased, they could analyze it further to establish its identity, The literature would be reviewed on the substances toxicological properties. Or perhaps the substance would undergo toxicological testing. Depending on what informa- tion is developed, regulatory agencies could then take appropriate action.

This approach tries to create a mechanism for quantitatively measuring uncharacterized substances in food. Proper guidelines are necessary since the cost of quantitatively identifying one substance can range from $\$ 10,000$ to $\$ 100,000$. Moreover, this monitoring approach requires sophisticated equipment for analyzing food samples. Also, the information generated by the analyses has to be computerized. Computer technology makes it possible to correlate and interpret chemical data to provide a continuous surveillance of the levels in food.

Pros: The uncharacterized monitoring approach is in the research stage at several laboratories in the country. If it is successfully developed, this type of monitoring would reduce the time during which the public is exposed to high concentrations of uncharacterized environmental contaminants in food. Kepone, for example, was polluting the James River, and people were eating kepone-contaminated fish for several years before the chemicals presence was discovered. With an uncharacterized monitoring system, kepone may have been detected years earlier.

Cons: This combination of sophisticated instruments, dependence on computers, and highly trained personnel is expensive. And the "hardware" needed is generally not found in Federal or State monitoring laboratories.

\section{OPTION 4--IMPROVE FEDERAL RESPONSE TO NEW CONTAMINATION INCIDENTS}

All of the major food contamination incidents have been marked by confusion. This stems from the involvement of three Federal agencies-FDA, the U.S. Department of Agriculture (USDA), and the Environmental Protection Agency (EPA) - in the monitoring and regulation of environmental contaminants in food. To cut down on confusion and to improve delivery of Federal technical assist- ance, Congress could choose to designate a lead agency or establish a center for the collection and analysis of data.

\section{Option 4A-Designate a Lead Agency}

The problem of conflicting Federal assistance efforts was dramatized by several recent incidents including the most recent PCB 
contamination. This sort of situation is not unique, Official reactions following the polybrominated biphenyl (PBB) episode in Michigan and the kepone incident in Virginia were similar.

The lead agency would serve as a clearinghouse for all information coming from and going to States. FDA would be the most likely candidate for lead agency when food contamination is suspected.

Pros: With a clearly delineated lead agency, States suspecting contamination of food would have one reliable source of technical assistance. Conflicts of opinion would be settled internally, and public statements and technical assistance provided with less ambiguity.

Cons: Designation of a lead agency might decrease the amount of technical expertise made available to the States. It would require the lead agency to develop new agreements with the other two agencies, During a food contamination crisis the lead agency would need to coordinate responses from the other agencies.

\section{Option 4B--Establish a Center to Collect and Analyze Toxic Substances Data}

Major delays in protecting the public from environmental contaminants in food now result from the time-consuming process of generating sufficient data on a substance's toxicity and dispersal in the food supply. Congress could overcome this problem by setting up a new technical center.

Such a center would be able to rapidly assemble technical teams skilled in the identification and analysis of organic, inorganic, and radioactive substances. A team would consist of a multidisciplinary group of experts, including chemists, toxicologists, food and animal scientists, epidemiologists, biochemists, biostatisticians, medical doctors, and others. Its mission would be to identify the cause of an actual or potential contamination incident, and assess the possible environmental and human health impacts. It would have no regulatory function.

The group would be able to mobilize within 24 hours. It would be the lead Federal organization that affected States could initially contact. In the wake of an episode, the team could be responsible for followup scientific research that would lay the groundwork for epidemiological studies of the exposed population. It would also be able to conduct a range of short-term toxicological tests to determine the mutagenicity and potential carcinogenicity of uncharacterized substances. It would be able to examine an exposed population for any adverse health effects from ingesting a particular contaminant in food.

The new technical center, in essence, would be similar to the Center for Disease Control (CDC). It would have the same capabilities in chemical epidemiology as CDC has in infectious disease. The new center could be given responsibility for investigating all toxic substance problems in the United States, not just those limited to food. If it were placed under CDC, it could build on work now underway in that organization's Environmental Hazards Unit. Furthermore, the center would be able to capitalize on the longstanding relationships between CDC and the States. Since the center's sole mission would be the development of information on environmental contaminants, it would not be linked to regulatory decisions.

As an alternative, the center might be located within FDA's National Center for Toxicological Research or another Federal laboratory with existing analytical capabilities. It could then use the laboratory's equipment and trained personnel for investigative analytical chemistry and toxicology.

It would be better not to locate the center and the investigation teams in a regulatory agency with direct responsibilities for food. This would ensure that there is no possible conflict of interest between its job of factfinding and the agency's responsibility for regulating, By distancing itself from the regulatory process, the new center team would 
strengthen its credibility with the media and the public.

Pros: Accurate scientific information on the nature and extent of food contamination has to be generated quickly when an incident occurs. Individual States often lack the scientific expertise to develop such data. The Federal Government does have the necessary capabilities, but the resources are scattered in several agencies with differing areas of authority. Such a dispersal of expertise hinders the gathering of urgently needed information following a contamination episode. Jurisdictional problems crop up, and States find they have to deal with more than one agency. The resulting delays slow the making of necessary health, environmental, and regulatory decisions.
Reaction time would be shortened and duplication of effort reduced by a technical or toxic substances investigation team which could react quickly in emergencies.

Cons: There is no assurance that a special team could generate information more quickly than is now the case. If the center were not part of a regulatory agency, it might not only grow out of touch with the needs but also could duplicate the investigatory work of the agency. Furthermore, the potential for an adversary relationship exists.

It could be argued that better coordination among FDA, USDA, and EPA would accomplish the same goals without the expense of establishing a new research center. 
Appendixes 


\section{Substances Whose Production or Environmental Release Are Likely to Increase in the Next 10 Years*}

by Clement Associates, Inc.

OTA requested Clement Associates to develop a list identifying new chemical substances likely to be manufactured in the near future and known chemicals likely to pose an increased burden to the environment because of increased production, new applications, or new technological developments, including new energy technology. For these projections, several factors were considered, including market trends, Federal regulatory activity, available substitutes for recently banned or restricted chemical substances, and consumer needs.

During the development of the approach to this phase of the project, certain problems and limitations became apparent. The nature of chemical substances under research and development but not yet introduced to the market is usually closely guarded proprietary information and therefore not available. In addition, there are no data systems which bring together chemical information to facilitate the retrieval of necessary data. An approach was developed to obtain a maximum amount of information in a limited amount of time.

Sources, including personal contacts, were identified for information on new chemicals or chemicals whose production, use, and release to the envirommenlikely to increase sharply because of future needs. These sources of information are listed in the reference list.

Following are the list of chemicals and a brief indication of why these chemicals were selected.

\section{CHEMICALS LIKELY TO HAVE SIGNIFICANTLY GREATER ENVIRONMENTAL RELEASE IN THE NEAR FUTURE}

Chemicals Whose Production and Use Are Likely to Increase Sharply

Because of Future Needs

\section{Soluble Polymers}

Soluble polymer "completing" agents are being developed to remove toxic metals from waste waters or remove radioactive metals from nuclear waste fluids. Polyethylene glycol and its

\footnotetext{
*Excerpt from OTA Working Paper entitled "Priority Setting of Toxic Substances for Guiding Monitoring Programs." A complete copy of the paper can be obtained through the National Technical Information Service. (See app. I.)
}

derivatives are the most versatile of the soluble polymers. Others are polyethyleneimine, polyvinyl sulfonic acid, polyacrylic acid with such chelating groups as thiourea, 8-hydroxyquinoline, iminodiacetic acid and hydroxyciniline, and polymers based on acrylic acid. (C\&EN, Mar. 27, 1978, p. 24)

\section{Organic Floceulants}

The principal organic flocculants are polyacrylamides, polyamides, and polyepichlorohydrins. Stiffening waste treatment regulations to reduce sludge are making flocculants more attractive. The chemicals make up a $\$ 100$ million per year 
market that may double within the next 5 years. These polymers may also be used for enhanced oil recovery if the price of oil would rise to make enhanced recovery economical. (C\&EN, Jan. 23, 1978, p. 9)

\section{Multifunctional Acryiates}

The use of radiation curing (ultraviolet or electron beam) is rapidly increasing. Radiation curing contributes significantly to critical energy savings and pollution abatement. Demand for the following multifunctional acrylates used for ultraviolet inks and coatings is expected to increase: pentaerythritol triacrylate, 1,6-hexanedioldiacrylate, trimethylolpropane triacrylate, and tetraethylene glycol. (C\&En, Jan. 23, 1978, p. 12, Celanese Chemical Co. Advertisement)

\section{Polyester Resin}

Concern about energy will augment the growth of lightweight polyester resin in the United States through the 1980's. The need for lighter weight materials in the automotive market, as well as the demand for corrosion-resistant products in many areas, will increase the demand for polyester resin at an average of 9.2 percent a year through 1987. Polybutylene terephthalate (PBT) and polyethylene terephthalate (PET) will be among the fastest growing polyester resins. PBT and PET are expected to grow from a combined demand of 38 million lbs in 1977 to 322 million lbs by 1987-an average annual growth rate of 24 percent. PBT is currently the most widely used thermoplastic polyester resin. However, it is expected that PET production will increase more rapidly, overtaking PBT production. The total polyester resin market is expected to rise 140 percent in the next 10 years. other uses and average annual projected production increases from 1977 to 1987 include unsaturated reinforced polyester (9. 1 percent), thermoset surface coatings (5 percent), cultured marble and other unsaturated thermoses (4.0 percent), strapping (mostly scrap, 28 percent), and fibers (4.0 percent). (C\&EN Jan. 30, 1978, p. 11)

\section{Zeolites}

Zeolites (aluminosilicates) hold promise as detergent builders, A 25-million-lb market in 1977 has a prospect of a possible 400-million-lb market in 1982. Zeolites containing detergents perform "equivalent or roughly equivalent" to the phosphate-silicate formulations that now command 75 percent of the current heavy-duty powder market. Environmental problems will continue to push phosphate out of the heavy-duty home laundry market. Zeolite $4 \mathrm{~A}$ is a cubic crystalline sodium aluminosilicate with the formula

\section{$\mathrm{Na}_{2} \cdot \mathrm{Al}_{2} \mathrm{O}_{3} \cdot 2 \mathrm{SiO}_{2} \cdot 45 \mathrm{H}_{2} \mathrm{O}$.}

\section{Textile Chemicals}

The market for textile chemicals is expected to top the billion-dollar market by 1980. Examples are:

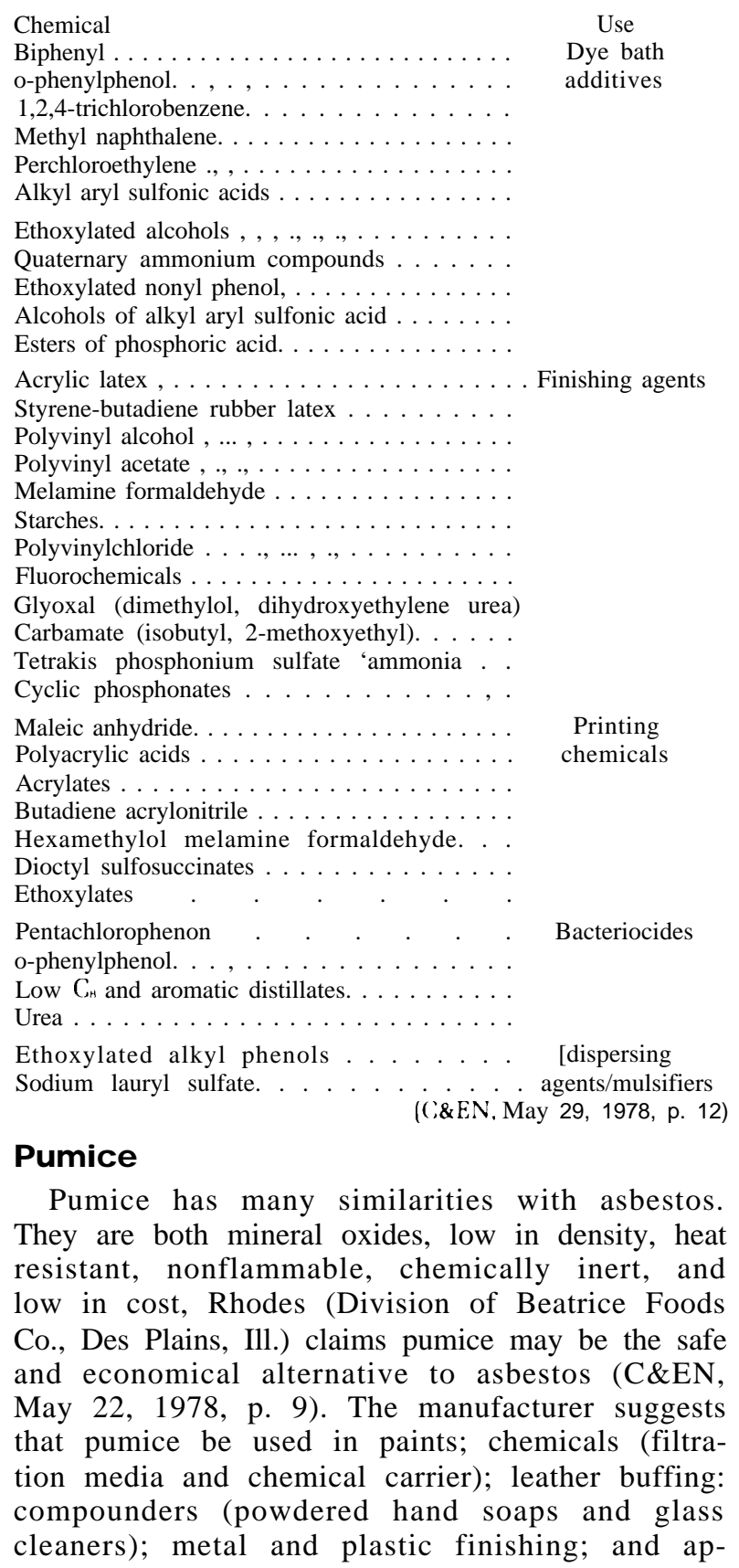


placations in the dental, rubber, glass, furniture, electronics, and pottery industries.

\section{Polyvinylacetates}

Acrylic and acetate resins compete in the market as textile and binder emulsions for nonwoven fabrics. Currently, acrylics dominate both of these markets by two-thirds of the total, Polyvinylacetates make up only 14 percent of the market, with the remainder going to other resins. New technical developments may change the status quo. By 1987, acetates could surpass acrylics in many quality paint and textile markets. (C\&EN, Mar, 20, 1978, p. 11)

\section{Hydrazine and Its Derivatives}

Originally used in rocket fuels, today hydrazine and its derivatives are commercially used in herbicides, pesticides, blowing agents for plastics, and water treatments. Its consumption is growing by 15 percent per year according to Olin Chemicals' advertisement in C\&EN.

\section{Olefinic Thermoplastic Elastomers}

Olefinic thermoplastic elastomers are also called TPO rubbers and were introduced in 1973, having a market volume of 1.5 million lbs. It is anticipated that the demand for TPO rubber will reach 44 million lbs by 1980 and that uses in automobiles will account for more than half of it. Mechanical goods and wire and cable uses account for 12.7 and 18,2 percent of TPO demand, respectively. Olefinic thermoplastic elastomers are also used by carmakers in electrostatically paintable body filler panels, air deflectors, and stone shields. Recently they have been used as sound deadening material on diesel-powered vehicles, jacketing and insulating material for wire and cable coating, and many custom-molded and extruded mechanical goods. (C\&EN, Oct. 23, 1978, p. 9)

\section{Silicones}

New markets for silicones as PCB replacements in electrical transformers, in brake fluids, and as elastomers are developing. Methylchloride is used as an intermediate in the production of silicones, Other methylating agents could be used to replace methyl chloride in most applications, but the potential substitutes (e.g., dimethyl sulfate, methyl bromide, methyl iodide) are much more expensive and have toxicity and handling problems which make them less desirable.

\section{Chlorobromination}

Bromine chloride has been shown to be more effective as a disinfectant than chlorine in field trials. It is a heavy red liquid and 12 times more soluble in water than chlorine. Its vapor pressure is one-third as great. Bromine chloride completely hydrolyzes almost immediately to hypobromous acid and reacts with ammonia in sewage to form bromoamines. It is believed that chlorobromination is the best alternative to current chlorination practices

\section{Polyethyleneterephthalate}

DuPont is continuing a vigorous effort to develop a market for molded plastics made from conventional forms of thermoplastic polyethylene terephthalate. (See also Polyester resin, ) This resin has a multibillion-pound demand as polyester fibers and films and is finding a large market in plastic beverage bottles. DuPont's trade name for PET is Rynite. Rynite's prime targets will be metals replacement, especially in automobiles.

\section{Chemicals Produced or Released Due to Energy Development Technology}

\section{Coal Liquefication and Gasification Program}

The conversion of coal to liquid to gaseous hydrocarbons for fuel technologies will result in the release of many chemicals to the environment. The contaminants may enter the environment via two pathways: 1) emission into the atmosphere with the consequent potential for long-range transport and 2) direct discharge via runoff and leaching into the aquatic and terrestrial domain where impact might be expected to be more localized. Contaminants from coal combustion can be classified into three groups: 1) organic chemicals, 2) inorganic chemicals, and 3) radionuclides.

\section{Organic Contaminants}

Organic contaminants from coal-derived process can be placed into several categories as shown below.

\begin{tabular}{|c|c|}
\hline Cintegory & Exomple \\
\hline Acids and anbydrides & Maleir anhydride \\
\hline & Benzoic acid \\
\hline Amines. ... & Aniline \\
\hline & Alpha and beta naphthylamines \\
\hline & Methylaniline \\
\hline & Benzidine \\
\hline arbonyl compoumeds & Formaldehude \\
\hline & Acetaldebyde \\
\hline
\end{tabular}




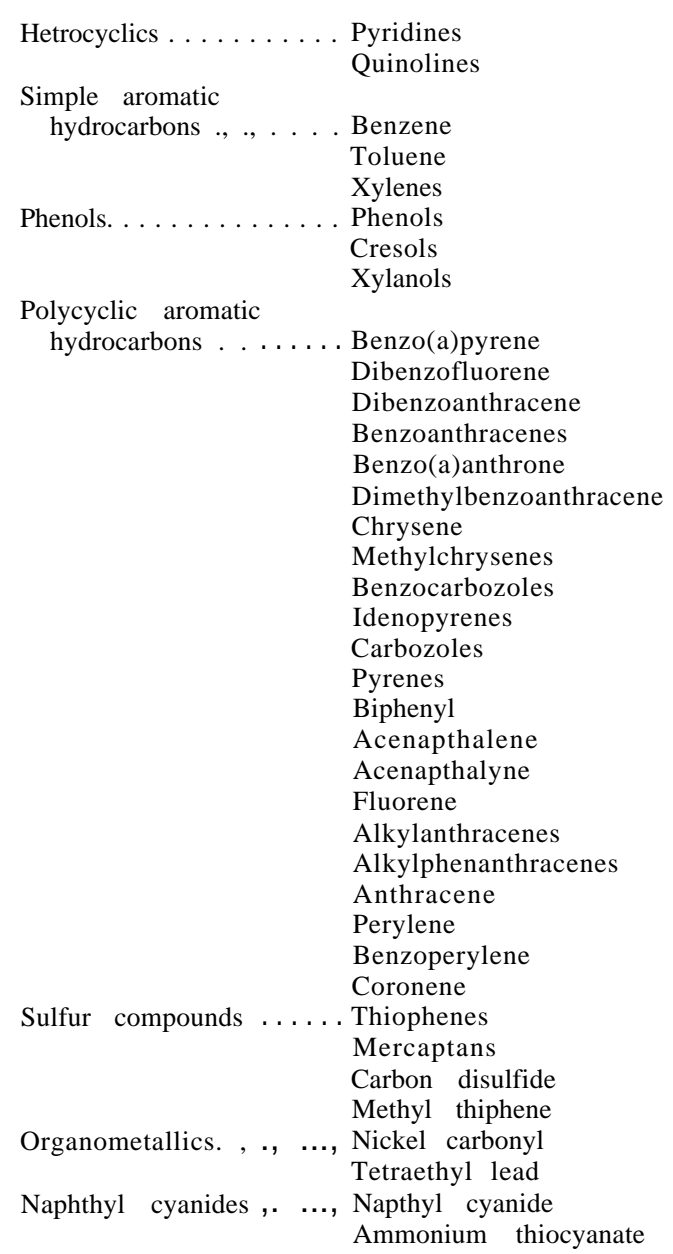

Trapped organic compounds and aromatic units in coals that were isolated, separated, and identified by gas chromatography and mass spectrometry are shown in table A-1.

\section{Inorganic Chemicals}

\begin{tabular}{|c|c|c|}
\hline \multirow{4}{*}{$\begin{array}{l}\text { Category } \\
\text { Acids . . }\end{array}$} & \multicolumn{2}{|l|}{ Examples } \\
\hline & \multicolumn{2}{|c|}{ Sulfuric acid } \\
\hline & \multicolumn{2}{|c|}{ Nitric acid } \\
\hline & \multicolumn{2}{|c|}{ Hydrochloric acid } \\
\hline \multirow[t]{2}{*}{ Chromium salts } & \multicolumn{2}{|c|}{ Chromium chloride } \\
\hline & \multicolumn{2}{|c|}{ Chromium sulfide } \\
\hline Sulfur compounds & Hydrogen sulfide & Carbon disulfide \\
\hline \multirow[t]{12}{*}{ Trace elements. . ., } & Antimony & Manganese \\
\hline & Arsenic & Mercury \\
\hline & Barium & Molybdenum \\
\hline & Beryllium & Nickel \\
\hline & Bismuth & Selenium \\
\hline & Cadmium & Silver \\
\hline & Chromium & Tellurium \\
\hline & Cobalt & Thallium \\
\hline & Copper & Tin \\
\hline & Fluorine & Uranium \\
\hline & Gallium & Vanadium \\
\hline & Lead & Zinc \\
\hline
\end{tabular}

\begin{tabular}{|c|c|}
\hline Fine $p$ & Sulfur particulate \\
\hline & Respirable coal dusts \\
\hline & \\
\hline & soots \\
\hline Gasses & Carbon monoxide \\
\hline & Sulfur dioxide \\
\hline & Sulfur trioxide \\
\hline & Sulfur tetraoxide \\
\hline & Nitrous oxide \\
\hline
\end{tabular}

\section{Radionuciides}

Emissions from a 100-MW electricity generation powerplant that burns coal at a rate of approximately 100 tons per hour are estimated to contain $1 \mathrm{ppm}$ of uranium and $2 \mathrm{ppm}$ of thorium, A plant of this size is expected to release 1 percent of its fly ash to the atmosphere. Under these conditions thorium-228 and -232, radium-224, and lead-212 each contribute approximately $5 \times 10^{-3}$ $\mathrm{Ci}$ per year; uranium-234 and -238 , thorium-230 and -234 , radium-226, lead-210, polonium-210, and bismuth-2 10 each contribute approximately 8 $x 10^{-3} \mathrm{Ci}$ per year; uranium-235 and praseodymium-231 both contribute approximately $3.5 \mathrm{x}$ $10^{-4} \mathrm{Ci}$ per year; and radon-220 and -222 together account for approximately $1.2 \mathrm{Ci}$ per year. (ERDA report 77-64, August 1976)

Radioactive emissions of bituminous coals have a high uranium content (75 ppm).

\section{Solar Heating and Cooling of Buildings}

Water contamination can occur in solar-heated domestic hot water systems at heat exchanger interfaces. Serious health consequences could be expected if the contaminated water is ingested. Water contamination could result from the heat exchange fluids themselves, or in water-based systems from such additives as:

Corrosion inhibitors - Chromates, berates, nitrates, nitrites, sulfates, sulfites, arsenates, benzoate salts, various triazoles, silicates, and phosphate compounds.

Freeze protestants-Glycols.

Heat transfer fluids-Paraffins, aromatic and other synthetic hydrocarbons.

Bacteriacides-Chlorinated phenols.

Solar collectors used in heating and cooling systems utilize organic chemical compounds as insulators that can emit highly toxic substances under overheat or fire conditions. Fumes usually consist of simple starches and phenolic compounds: ammonia, hydrochloric acid, hydrofluoric acid, toluene diisocyanate (TOI), and hydrogen cyanide. 
Table A-1 .-Trapped Organic Compounds and Aromatic Units in Coal

1. Straight.chain hexane

2. 2. hexene

3. Dimethylbutane, methyl. cyclopentane

4. cyclohexane

5. Cr-alkene (B)

6. Benzene

7. Thiophene

8. Cr-alkane (B), C-alkene (B)

9. $C$-alkadiene $(B)$ or $C$. alkyne (B)

10. Cyclohexene

11. C.-alkane (B)

12. Dimethylcyclopentane

13. 2-and 3-methylhexanes

14. Heptene

15. 2.3-dimethyl-2-pentene

16. Methylcyclohexane

17. Dimethylhexane

18. Heptyne

19. Trimethyipentane

20. Methylheptane

21. Methylheptene

22. Trimethylcyclopentane

23. 1-methylcyclohexene

24. Toluene

25. Dimethylcyclohexane

26. Methylthiophene

27. $\mathrm{C}_{9}$-aikene $(\mathrm{B})$

28. Ethylcyclohexane

29. Trimethylcyclohexane

30. n-propyl andior isopropylcyclohexane

31. C:-alkylcyclopentane (?)

32. $C_{9}$-alkane (B). $C_{4}$-alkene

33. C.alkyne (?) andior $C_{4}$. alkadiene (?)

34. Ethylbenzene

35. Dimethylthiophene

36. $m$, and $\rho \cdot x y l e n e$

37. $o \cdot x y l e n e$

38. $\mathrm{C}_{9} \cdot$ alkene $(\mathrm{B})$

39. tetramethylcyclohexane

40. $C_{1 n}$-alkene (B)

41. C:-alkyicyclohexane

42. Diethylcyclohexane (?)

43. C ,alkane (B). C , alkene (B)

44. C 10.alkene
45 Ethyloctane (?)

46 Trimethylthiophene

47 Propylbenzene

48 Methyl ethylbenzene

49 Trimethylbenzene

50 C r-alkene (B)

$51 \mathrm{C}_{\text {-alkylbenzene }}$

52. $C_{1}$.alkene $(B)$ and $C_{1}$. alkylbenzene

53 Tetramethylbenzene

54 1-methyl-4-isopropyl-3.

cyclohexene (?)

55 Methylindan

56 C,-alkene (B)

57 Dimethylindan

58 C.-alkylbenzene

59 Tetralin

$60 \mathrm{C}$,-alkylbenzene

61. C., alkene,

$\mathrm{C}_{6}$-alkylbenzene

62 Naphthalene

$63 C_{1}$-alkane (B). $C_{*}$-alkene (B)

64 2-methylnaphthalene

65 1.methylnaphthalene

66 C-alkyldecalin

$67 \mathrm{C}$ :-alkyldecalin

68 Trimethylindan

69 Tetramethylindan and/or trimethyltetralin

$70 \mathrm{C}_{4}$-alkylcyclohexane

71 Biphenylene

72 2-ethylnaphthalene

73 1.ethyinaphthalene

74 Dimethylnaphthalene

75 Cadinane (4.10-dimethyl.7. isopropyldecalin)

76 Dihydrocadinene ( $\mathrm{T}$ ) $\mathrm{C}_{9}$-alkylcyclohexane

77 Selinane and eremophilane hydronaphthalenes)

78 Dihydroselinene (T) and/or dihydroeremophilene (T)

79 Dihydrocadinene ( $\mathrm{T}$ )

$80 \mathrm{C}^{-}$-alkylindan

81 Methylcenaphthene. 2(?). isopropylnaphthalene

82 Diphenylmethane
833 or 4-methylbiphenyl

84 Cr-alkylbenzene

85 Tetramethylindan

$86 \mathrm{C}_{13} \mathrm{H}_{2 \mu}$-sesquiterpenoid nydrocarbon (?)

$87 \mathrm{C}_{\text {s-alkyltetralin }}$

88 Methyl.ethylnaphthalene and/or

trimethylnaphthalene

89 Trimethylnaphthalene

90 Fluorene

91 1.6-dimethyl-4-isopropyl-1. 2-dihydronaphthalene $(T)$

92 Iso-butylnaphthalene. trimethylnaphthalene

93 1-methyl-4-isopropyl naphthalene

94 Eudalene (1-methyl-7. isopropyl-naphthalene)

$95 \mathrm{C}_{\text {s-alkyltetralin (?) }}$

96 1-methyl-2. propylnaphthalene $(T)$

97 Cadalene (1,6-dimethyl.4 isopropyl-naphthalene)

98 Tetramethylnaphthalene

99. 1.4-dimethyl-6 (?).

isopropyl-naphthalene. $\mathrm{C}_{6}$ alkyltetralin ( $T$ )

$100 \mathrm{C}_{t}$-alkyltetralin (T)

101 1,2.5.7-tetramethyl. naphthalene

102 Pristane

103 Methylfiuorene

104 Pentamethylnaphthalene

105 Dibenzothiophene

106 Trimethyloctahydro phenanthrene

107 Vethyltetrahydrophenan :hrene ( $T$ )

108 Thenanthrene

10y. $\mathrm{C}, \mathrm{H}:$ (m/e 191 , base peak), $\mathrm{C} \cdot \mathrm{H}_{\mathrm{o}}\left(\mathrm{M}^{+}\right)$tricyclic terpenoid (?)

110. Dimethyltetrahydrophe nanthrene ( $T$ )

111. Ethyltetrahydrophenan. threne (T)

112. Anthracene

113. Naphthofuran
114. Methyldibenzothiophene

115. $\mathrm{C}_{9}$-alkyltetralin (?)

116. $\mathrm{C}_{20} \mathrm{H}_{32}$ (abietadiene (?))

117. $\mathrm{C}_{10} \mathrm{H}_{10}$ (tricyclicditerpenoid (?))

118. 2-and/or 3-methylphenan. threne

119. 1. and/or 9-methylphenanthrene

120. 1.7-dimethylphenanthrene

121. Dimethyldibenzothiophene

122. C.o.alkyltetralin (or $\mathrm{C} .$. alkylindan) (?)

123. Dehydroabietene $(4.20$. dimethyl-13-isopropyl-8H. phenanthrene)

124. Dimethylphenanthrene andlor dimethylanthracene

125. Dehydroabietane

126. $\mathrm{C}_{20} \mathrm{H}_{12}$ (tricyclic diterpenoid) (?)

127. Fluoranthene

128. C, ,alkyltetralin (?)

129. Abietatetraene (T) (trimethylisopropyl.6 H. phenanthrene)

130. 1,2,3,4-tetrahydroretene (T)

131. Methyl-ethylphenanthrene and/or trimethylphenan. threne

132. Pyrene

133. Simonellite

134. Retene (1-methyl-7. isopropylphenanthrene)

135. 1.2-benzofluorene

136. 2.3-benzofluorene

137. 3,4-benzofluorene

138. Methylpropylphenanthrene andior dimethylethylphenan threne

139. Methylbenzofluorene

140. Tetramethylphenanthrene andior tetramethylan. thracene

141. Chrysene and/or triphenylene

$B=$ branched $T=$ identification tentative $?=$ identification uncertain

\section{Fuels From Biomass}

Thermochemical biomass conversion can produce gases, tars, oils, and unconverted residue (char) and ash, depending on the particular conversion process. Thermochemical reactions generated sulfur-containing $\left(\mathrm{H}_{2} \mathrm{~S}, \mathrm{COS}, \mathrm{CS}_{2}, \mathrm{SOX}\right)$ and nitrogen-containing ( $\left.\mathrm{HCN}, \mathrm{NOX}, \mathrm{NH}_{3}\right)$ gases.

Water can be affected by the residuals produced from thermochemical conversion. Low-molecular weight oils, phenols, leachates from char and ash residues, and scrubber solution runoff may enter water bodies by direct discharge or by percolation to subsurface waters from evaporation points. 


\section{REFERENCE LIST FOR SUBSTANCES WHOSE PRODUCTION OR ENVIRONMENTAL RELEASE ARE LIKELY TO INCREASE IN THE NEXT 10 YEARS}

Chemical Marketing and Economics Abstracts. The Division of Chemical Marketing and Economics of the American Chemical Society (ACS) presents papers at ACS national meetings on subjects related to the responses of the chemical industry to economic changes as well as responses of the financial community to changes in the chemical industry. Abstracts of these papers are published by ACS.

A Study of Industrial Data on Candidates for Testing. This document, published by the U.S. Environmental Protection Agency, Office of Toxic Substances (EPA Contract No. 68-01-4109, November 1976) contains market forecasts for 10 major classes of chemicals (109 individual chemicals) with an annual production greater than or equal to 1 million lbs. Chemicals that are used exclusively as drugs or pesticides are not included. The market forecasts include: 1) a discussion of production and trade statistics, 2) consumption patterns, whenever possible, 3) growth trends, 4) a brief summary of current uses as well as potential new applications, and 5) growth trends in endmarket consumption. A discussion of possible substitutes for some of the chemicals is also included.

Environmental Development Plans. The Environmental Development Plans (EDPs) published by the U.S. Department of Energy (March 1978) were conceived and prepared as basic documents to assist in planning and managing environmental programs of energy technology development. Approximately 30 EDPs covering major developing energy technologies were prepared.

A Review of Current Information on Some Ecological and Health-Related Aspects to the Release of Trace Metals Into the Environment Associated With the Combustion of Coal. This document by Merrill Heit is a technical report (HASL-320) from the Health and Safety Laboratory, Energy Research and Development Administration, New York, N.Y. 10014, reviewing the literature on one class of pollutants. Information on the environmental levels, ecological effects, and potential toxicity to man of 35 elements that may be released into the environment by coal combustion or gasification is presented.

Fossil Energy Update. This monthly journal compiled by the Department of Energy lists abstracts of current scientific and technical reports, journal articles, conference proceedings, theses, and monographs on all aspects of fossil energy, including factors involving the environment, health, and safety.

Chemical Engineering News. The Chemical Engineering News is a weekly publication of the American Chemical Society that contains relevant information in such sections as "Chemical World," "This Week," "Business Concentrates, " and "Science/Technology," and in profiles on selected chemicals.

Chemical Marketing Reporter. This weekly, published by Schnell Publishing Company, Inc., contains information on chemical market reports and profiles on selected chemicals.

Trapped Organic Compounds and Aromatic Units in Coal. This article, published by Tyoichi Hayatsu et al. in Fuel 57:541 (1978), contains a detailed analysis of the organic constituents of three coals: a lignite, a bituminous, and an anthracite. Organic compounds trapped in the coal matrix, residuals, and products of the original coalification process were described.

Personal Communications With Several Organizations, Including the Chemical Development Association and Chemical Marketing Research Association. 


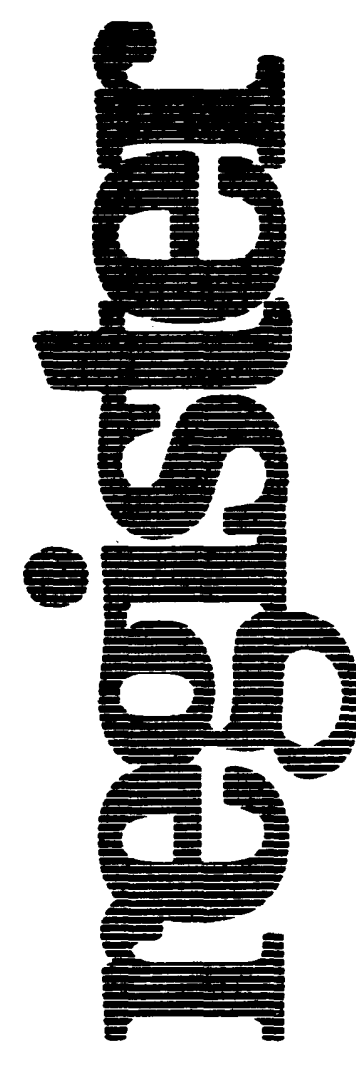

Friday

June 29, 1979

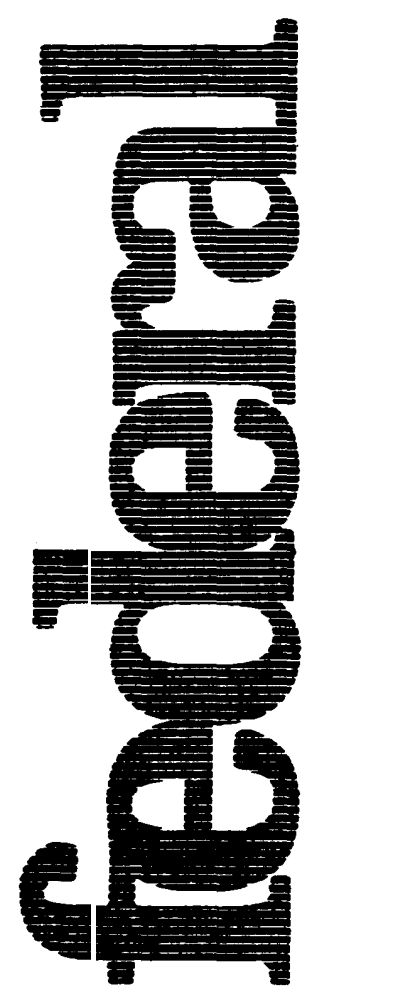

Department of Health, Education, and Welfare

Food and Drug Administration

Polychlorinated Biphenyls (PCB's);

Reduction of Tolerances 
DEPARTMENT OF HEALTH, EDUCATION, AND WELFARE

Food and Drug Administration

\section{CFR Part 109}

[Docket No. 77N-0080]

Polychlorinated Biphenyls (PCB's); Reduction of Tolerances

agency: Food and Drug Administration. Action: Final Rule.

summary: The Food and Drug

Administration [FDA) is reducing the tolerances for unavoidable residues of the industrial chemicals polychlorinated biphenyls (PCB's) in several classes of food. Specifically, the agency is reducing the tolerances in milk and dairy products from 2.5 parts per million (ppm) to $1.5 \mathrm{ppm}$ (fat basis), in poultry from 5 ppm to 3 ppm (fat basis), in eggs from 0.5 $\mathrm{ppm}$ to $0.3 \mathrm{ppm}$, and in fish and shellfish from $5 \mathrm{ppm}$ to $2 \mathrm{ppm}$.

DATES: Effective August 28, 1979; objections on or before July 30, 1979. ADDRESS: Written objections to the Hearing Clerk (HFA-305), Food and Drug Administration, Rm. 4-85, 5000 Fishers Lane, Rockville, MD 20857. FOR FURTHER INFORMATION CONTACT: Howard N. Pippin, Bureau of Foods (HFF-312), Food and Drug

Administration, Department of Health, Education, and Welfare, 200 C St. SW., Washington, DC 20204,202-24\$3092.

SUPPlementary information: In the Federal Register of April 1,1977 [42 FR 17487), FDA proposed to reduce the temporary tolerances for unavoidable residues of PCB's in several classes of food. The agency received over 100 comments on the proposal from interested individuals, consumer groups, businesses, trade associations, State government agencies, and others. The agency has considered these comments and is now issuing a final order reducing the PCB tolerances as originally proposed. Following a brief discussion of the background, this document will respond to the comments the agency received and explain the agency's reasons for adopting the reduced tolerance levels.

\section{Background}

PCB's are a class of toxic industrial chemicals that have become persistent and ubiquitous environmental contaminants as a result of past widespread, uncontrolled industrial use, As explained in the preamble to FDA's proposal initiating this rulemaking proceeding (see 42 FR 17489), one result of PCB contamination of the environment has been contamination of certain foods. In the Federal Register of July 6,1973 (38 FR 18098), FDA issued regulations to deal with the problem of PCB contamination of food. Among those regulations was one establishing temporary tolerances for unavoidable PCB residues in various categories of food. Those original tolerances are now codified in $\$ 109.30$ (21 CFR 109,30). The order FDA is issuing in this document reduces certain of those tolerances.

FDA's authority to issue tolerances for unavoidable food contaminants is derived from sections 402(a)(2)(A) and 408 of the Federal Food, Drug, and Cosmetic Act (the act) (21 U.S.C. $342(a)(2)(A)$ and 346). Section 402(a)(2)(A) deems food adulterated, and thus prohibited from interstate commerce, if it contains "any added poisonous or added deleterious substance" that is unsafe within the meaning of section 408 , Section 408 deems any added poisonous or deleterious substance to be unsafe unless its presence in the food is required in the production thereof or cannot be avoided by good manufacturing practice. Section 408 also authorizes the agency to promulgate regulations limiting the quantity of such a required or unavoidable substance that can be present legally in food. Such limits, called tolerances, are to be set by FDA at the level found necessary to protect the public health, taking into account the extent to which the . substance is required or unavoidable and the other ways the consumer may be affected by the same or other poisonous or deleterious substances, Once a regulation establishing a tolerance has been promulgated for a particular poisonous or deleterious substance, food containing that substance in an amount exceeding the tolerance is deemed adulterated under section 402(a) (2)( A]

One of the primary purposes of section $\mathbf{4 0 6}$ of the act is to enable FDA to deal effectively with environmental contaminants such as PCB's, These substances often enter food as a result of events beyond the reasonable control of the food manufacturer or processor and, once in the food, usually cannot be removed by good manufacturing practice. For example, in the case of PCB's, some species of fish have become contaminated to varying degrees as a result of the dumping of PCB-containing industrial waste into the nation's waters (see the Federal Register of March 18, 1972 (37 FR 5705)]. Once the contamination occurs, there is little that can be done to remove the PCB's from the water or from the fish; their presence is, in that sense, unavoidable. Because the initial contamination of fish with PCB's cannot be avoided (nor the PCB's processed out), the only way to avoid PCB's in fish is to remove fish from commerce if it contains PCB's above a given tolerance level. The degree of avoidance accomplished by this method is, of course, a function of the level at which the tolerance is set, In this way, it is theoretically possible to avoid PCB's in fish absolutely by removing from commerce all fish that contain any amount of PCB's.

Section $\mathbf{4 0 6}$ of the act authorizes FDA to make a practical judgment in dealing with such environmental contaminants: Based on an assessment of the degree to which the contaminant poses a threat to consumers, the agency can decide to. tolerate the contaminant's presence in food up to a level the agency considers appropriate to protect the public health, taking into account, among other factors, the extent to which the presence of the contaminant is unavoidable, In making this judgment, the agency's paramount concern is protection of the public health: The tolerance cannot be set above the level. the agency finds necessary to protect the public health adequately. But in determining what tolerance level provides an adequate degree of public health protection, FDA is required by section $\mathbf{4 0 6}$ to consider the extent of unavoidability-in the case of PCB contamination of fish, the amount of PCB-contaminated fish that must be disposed of to reduce human exposure to PCB's to a tolerable level, As a practical matter, of course, a tolerance, if it is to be enforceable, cannot be set below the level at which the contaminant can be reliably measured for enforcement purposes by available analytical methods.

The toxicological data available on PCB's make it clear that, in an ideal situation, it would be preferable not to have PCB's in food at any level. As discussed more fully below, the data do not permit the identification of any level of PCB exposure that can be said to provide an absolute assurance of safety, It is equally clear, however, that the reduction of PCB exposure from food sources to zero, or to a level approaching zero, would require elimination of large amounts of food, especially fish. Hence, in deciding the appropriate levels for PCB tolerances under section 408, FDA has had to make some extraordinarily difficult judgments. It has had to decide, in effect, where the proper balance lies between providing an adequate degree of public health 
protection and avoiding excessive losses of food to American consumers.

The comments received on the proposal reveal that by far the most controversial aspect of this rulemaking proceeding is the balancing judgment FDA made in proposing to reduce the PCB tolerance in fish from 5 to $2 \mathrm{ppm}$. Some comments argued that the proposed reduction would cause an excessive loss of food and significant adverse economic impact without providing any significant increase in public health protection. Other comments argued the converse, i.e., that the proposed reduction to $2 \mathrm{ppm}$ would not adequately protect the public health and that the tolerance should be reduced to $1 \mathrm{ppm}$ (the lowest level at which PCB residues in fish can be reliably measured fop enforcement purposes), despite the additional losses of food that a reduction to $1 \mathrm{ppm}$ would cause. In each case, the comments bolstered their arguments by contending that FDA has either overestimated or underestimated the toxicity of PCB's and the impact of the proposed reduction of the tolerance in terms of food loss and adverse economic consequences.

The comments criticizing the proposed reduction of the fish tolerance highlight the difficulty of the judgment FDA must sometimes make in establishing tolerances. Not only must FDA make a qualitative judgment about the proper balance between adequate public health protection and excessive loss of food, it also must often make that judgment on the basis of data that are incomplete, or even in dispute, and that can easily lead reasonable people to differing conclusions. As the comments illustrate, it is nearly always possible to conduct additional studies and investigations to refine further the knowledge of a substance's toxicological profile, the incidence and-degree of human exposure to it, and the impact a given tolerance reduction will have on the food supply. As an agency whose first responsibility is to protect the public health, however, FDA must act on the basis of the information available to it, even when the information is incomplete. Neither the agency nor the public can afford to wait until every uncertainty is resolved. See Ethyl Corp. v. Environmental Protection Agency, 541 F. 2d 1, 24-29 (D.C, Cir,) (en banc), cert. denied, 426 U.S. 941 (1976].

In the case of PCB's, even though there are obvious shortcomings in the available data, which are discussed below, FDA considers the data to provide a more than adequate basis for the exercise of its judgment in reducing the PCB tolerances. There would be no advantage in delaying this action because it will take years to resolve certain of the shortcomings in the data on PCB's, if they can be resolved at all. For example, no chronic toxicity studies have been performed on the specific, chemically distinct composition of PCB's found in fish residues. Even if such studies were begun immediately, it would be 3 to 4 years before results could be available. That plainly is too long to wait to take action necessary for the protection of the public health.

Because of the emphasis the comments placed on the proposed reduction of the fish tolerance, this document reviews the basis on which the reduction was proposed and explains why, after considering the comments, the agency has decided to promulgate the reduction as proposed. After discussing the fish tolerance and the major points raised about it in the comments, this document responds to the remaining comments received on other aspects of the proposal.

\section{The Tolerance for Fish and Shellfish}

In the preamble to the April 1, 1977 proposal, the agency discussed new toxicity data that had become available after the original PCB tolerances were promulgated in 1973 [42 FR 17488-9). In contrast to the data underlying the original tolerances, which consisted primarily of data from retrospective studies of humans in Japan who were exposed to high doses of PCB's and showed acute toxic effects from the exposure (42FR 17487-8), the new toxicity data consist primarily of animal studies showing an association between PCB exposure and serious subchronic and chronic toxicities, including adverse reproductive effects, tumor production, and, possibly, carcinogenicity, as well as effects on numerous biochemical systems [42 FR 17488-9). Although the data do not fully resolve such important questions as the carcinogenicity of PCB's, they lead to the conclusion that neither "no effect" nor "allowable daily intake" levels for PCB's can be established with any confidence and that, from a toxicological point of view, human exposure to PCB's should be reduced.

The preamble to the proposal also discussed data FDA had gathered on human exposure to PCB's, especially from dietary sources (42 FR 17489-90), These data show that the current incidence of PCB contamination of food has declined significantly in comparison to that on which the original PCB tolerances were based (see 37 FR 5705), Indeed, the new data show that fish are the only food group in which detectable levels of PCB contamination are now routinely found.

Based on the declining incidence of PCB contamination, which means that PCB's are avoidable in food to a greater degree now than they were earlier, as well as the new toxicity data suggesting chronic toxic effects, FDA decided the PCB tolerances should be reduced.

In the preamble to the proposal, the agency analyzed the new toxicity and exposure data as they bore specifically on the tolerance for PCB's in fish(42 FR 17492-3). The agency concluded that reduction of the tolerance from 5 to 2 ppm was necessary to protect the public health adequately, even though that reduction would result in the estimated loss of a minor percentage of marine fish (approximately 0.2 percent) and up to 25 percent of freshwater fish shipped interstate (the loss of marine and freshwater fish having a combined landed value of approximately $\$ 8$ million per year). The agency concluded that the increment of public health protection afforded at least theoretically by a further reduction of the tolerance to $1 \mathrm{ppm}$ did not justify such a reduction in light of the substantially greater loss of food that would result (a combined landed value, marine and freshwater. of approximately $\$ 18$ million per year).

As noted, a large majority of the comments on the proposal dealt with some aspect of the agency's proposal to reduce the fish tolerance to $2 \mathrm{ppm}$. Some of the comments agreed that the proposal struck a proper balance between the need to protect the public health and the need to avoid excessive loss of food. Other comments argued that the tolerance should be reduced to 1 ppm in light of the new toxicity data on PCB's, despite any additional loss of food that might result.

Most of the comments on the fish tolerance, however, were submitted by members of the fishing industry, by trade associations, and by agencies of State governments involved in commercial fishing matters, who argued that reduction of the tolerance to $2 \mathrm{ppm}$ is not justified. Some of these comments contended that the health hazard presented by occasional consumption of fish containing 5 ppm PCB's is not significant and that any reduction in risk to consumers accomplished by reducing the tolerance to $2 \mathrm{ppm}$ would be minor. These comments also argued that any such risk reduction would be outweighed by the resulting adverse economic consequences, which some argued would be far in excess of those cited by the agency in its proposal. In support of the latter argument, some of these comments estimated the impact a 
$2 \mathrm{ppm}$ tolerance would have not only on the commercial fish catch, but also on employment and income in the fishing and related industries and on recreational fishing. Arguing that the States would curtail recreational fishing in certain areas if the tolerance were reduced to $2 \mathrm{ppm}$, the comments projected large losses of sales among those supplying boats, licenses, tackle, and bait to sport fishers.

Due to the large volume of comments challenging the proposed reduction of the fish tolerance, the agency has carefully reassessed the justification for lowering the tolerance from 5 to $2 \mathrm{ppm}$. It has reviewed the toxicological data and has attempted to estimate in quantitative terms the degree to which lowering the tolerance would reduce risk to consumers. In addition, it has reexamined the question of how much additional loss of fish would occur as a result of the proposed reduction. Based on its reassessment, the agency concludes that reduction of the tolerance for PCB's in fish to $2 \mathrm{ppm}$ strikes the proper balance between the need to protect the public health and the need to avoid unnecessary loss of food. Hence, the reduced tolerance is being promulgated as proposed.

\section{A. Risk Reduction}

As noted earlier in this preamble, the proposal to reduce the fish tolerance was based in part on new toxicity data showing a relation between PCB exposure and an increased incidence of various subchronic and chronic toxic effects, including adverse reproductive effects, tumor production, and, possibly, carcinogenicity (42 FR 17487-9). The proposal itself noted certain factors that complicate the evaluation of PCB toxicity (e.g., varying degrees of toxicity among the several forms of PCB's, the presence of toxic impurities such as chlorinated dibenzofurans in commercial preparations of PCB's, the differences in chemical composition between commercial PCB's and PCB residues in fish, and varying susceptibilities of different animal species to the toxic effects of PCB's); these complicating factors were also pointed out in some of the comments received on the proposal,

Notwithstanding these factors, however, there is little genuine dispute over the fact that exposure to PCB's must be considered to pose a risk of serious, chronic toxic effects in humans. The toxicological judgment that flows from this fact-i.e., that a reduction in human exposure to PCB's will reduce this risk-was an important part of the agency's rationale for proposing to reduce the fish tolerance. Nothing in the comments and nothing discovered during FDA's reassessment of the toxicity data alters the validity of that fundamental judgment. The agency therefore concludes that it is important as a matter of public health protection to minimize human exposure to PCB's.

The real question raised by the comments is whether the degree of risk reduction accomplished by lowering the fish tolerance to $2 \mathrm{ppm}$ is sufficient to justify the increased loss of food that the lower tolerance will cause. This is an extremely difficult question because it is not now possible for toxicologists to quantify precisely, on the basis of toxicity data derived from animal studies, the risks posed to humans. Using classical toxicological methods, the most that can be done reliably is to make qualitative judgments about risks: A statistically significant increased incidence of adverse effects in animals is good evidence of a risk to humans, and, generally, the greater the incidence of effects in animals, the greater the risk to humans (Ref. 43). Having identified the risk of a chronic toxic effect from exposure to a substance, classical toxicological principles lead to the conclusion that reduction in exposure will reduce the risk (Ref. 44). Again, there is no evidence that these principles do not apply to PCB's.

Scientists have recently developed methods, incorporating mathematical extrapolation models, for making quantitative estimates of risks to humans based on toxicity data from animal studies. These risk assessment methods do not purport to quantify precisely the expected human risk, but rather attempt to estimate in quantitative terms an upper limit on the risk to humans that can be expected from a given level of exposure to a toxic substance, assuming humans are no more susceptible to the effects of the substance than are the most susceptible members of the animal species for which toxicity data are available. These risk assessments can be useful as a means of comparing risks at various exposure levels and illustrating the toxicological judgment that a reduction in exposure will reduce risk. Because of all the problems inherent in extrapolating from animal data to the expected human experience, however, the numbers produced by a risk assessment must be interpreted cautiously: They are estimates of upper limits on risk and, though potentially useful for comparative purposes, cannot be said to quantify actual human risk precisely. These assessments attempt to avoid underestimating human risk, but even that cannot be guaranteed. The Work Group on Risk Assessment of the Interagency Regulatory Liaison Group (IRLG) has recently prepared a report that discussers many of the principles involved in risk assessment.

As part of its review of the toxicological justification for reducing the fish tolerance, the agency has performed a risk assessment aimed at comparing the estimated risks associated with PCB exposure at the various levels of exposure that would result from different tolerance levels The written report on this risk assessment has been made a part of the record of this proceeding as Reference 45.

As explained in that report, the risk assessment involved the use of the most recent available data on the incidence of PCB contamination of fish to calculate the level of exposure to PCB's that could be expected to result from tolerance levels of 5,2, and $1 \mathrm{ppm}$ (Table 4, Ref. 45). These calculations were based on the assumption that under a given tolerance level, no fish containing PCB's in an amount above that level would be consumed, It is true, of course, that an FDA tolerance level directly affects only fish shipped in interstate commerce, but States often adopt FDA's tolerance levels for application to intrastate and recreational fishing. Thus, even if the exposure calculations used in the risk assessment (Table 4, Ref. 45) do somewhat overstate the absolute amounts of exposure reduction, they nevertheless demonstrate that a reduction of the PCB tolerance for fish would result in a significant reduction of PCB exposure [e.g., for heavy consumers of the affected species, reduction of the fish tolerance from 5 to $2 \mathrm{ppm}$ reduces exposure from an estimated 20.1 micrograms $(\mu \mathrm{g})$ per day to an estimated $14.9 \mu \mathrm{g}$ per day). Such significant reductions in PCB exposure from fish are especially important in terms of risk reduction because fish are the only food group in which detectable levels of PCB's are still regularly found.

Based on the calculations of exposure at various tolerance levels and toxicity data from animal studies, the agency used a linear extrapolation method to estimate the upper limits on certain risks posed by exposure to PCB's, This analysis resulted in estimates of significant potential risk to humans who consume PCB-contaminated fish on a continuing basis, especially fish contaminated at or above the $5 \mathrm{ppm}$ level (Tables 6 and 7, Ref. 45). For example, using the total malignancy data from the National Cancer Institute (NCI] Bioassay (Ref. 19), it is estimated 
Federal Register / Vol. 44, No. 127 / Friday, June 29, 1979 / Rules and Regulations

that the upper limit on the lifetime risk of cancer for heavy consumers of fish most affected by the tolerances is 9.8 incidence of cancer per 100,000 of the population, assuming the tolerance is 5 ppm; 7.2 per 100,000, assuming the tolerance is $2 \mathrm{ppm}$; and 4.4 per 100,000 , assuming the tolerance is $1 \mathrm{ppm}$ (Table 6 , Ref. 45). Stated another way, it is estimated that the upper limit on the number of new cancers per year among heavy consumers of fish most affected by the tolerances is 46,8 , assuming a tolerance of $5 \mathrm{ppm} ; 34.3$, assuming a tolerance of $2 \mathrm{ppm}$; and 21, assuming a tolerance of $1 \mathrm{ppm}$ (Table 7, Ref. 45).

As explained in the report (Ref. 45), the utility of this risk assessment for evaluating actual risk to humans from exposure to PCB's is extremely limited. This is due both to difficulties inherent in making such extrapolations from animals to humans and, perhaps more importantly in this instance, to gaps and uncertainties in the data available for this particular risk assessment. For example, the toxicity studies on which the risk assessment is based used commercial preparations of PCB's, which are chemically different from the PCB residues found in fish and which contain small amounts of highly toxic impurities (e.g., dibenzofurans) not known to be present in fish residues, Also, in making the exposure estimates required for the risk assessment, it was necessary to use existing data on the numerical distribution of PCB levels in fish and rely on the assumption that the effect of a given tolerance level is to remove from commerce all fish containing PCB's exceeding the tolerances. It is possible that neither the assumption nor the data precisely reflect what actually occurs.

For these reasons and others discussed in the report (Ref. 45), the risk assessment does not provide a basis for precise quantification of the amount of risk reduction accomplished by reducing the fish tolerance, Despite the limitations inherent in the risk assessment, however, the agency regards it as illustrative of the basic validity of the toxicological rationale for reducing the tolerance for PCB's in fish: Reduction of the tolerance will result in a significant reduction in risk among those who consume PCB-contaminated fish. FDA considers this risk reduction to be of significant public health value, even though it cannot be precisely quantified.

\section{B. Loss Of Food}

In the preamble to the proposal, the agency estimated that the loss of food from commercial channels resulting from a 2 ppm tolerance for PCB's in fish would be approximately $\$ 8$ million in landed value, compared to approximately $\$ 1$ million for the $5 \mathrm{ppm}$ tolerance and $\$ 18$ million for a $1 \mathrm{ppm}$ tolerance, The estimated $\$ 8$ million loss resulting from a $2 \mathrm{ppm}$ tolerance encompassed a negligible percentage of the marine-fish catch (about 0.2 percent) and about 25 percent of the freshwater catch (42 FR 17492),

The agency arrived at these figures by assuming that all fish containing PCB' above the tolerance would be removed from both interstate and intrastate commerce ("Economic Impact Assessment for Proposed Reduction of Temporary Tolerances for Polychlorinated Biphenyls in Food," Ref. 39). There are several difficulties inherent in this assumption. On the one hand, it may tend to overstate the loss because (a) some states may not apply FDA's reduced tolerance to intrastate fish, (b) some violative fish will be part of nonviolative lots, and (c) some violative lots may enter commerce undetected. On the other hand, it may tend to understate the loss because once the violative percentage of a given species reaches a certain level, commercial fishers may stop fishing that species altogether. Some of the comments cited these difficulties in support of arguments that FDA had either overestimated or underestimated the amount of fish that would be lost as a result of a $2 \mathrm{ppm}$ tolerance. Despite its acknowledged limitations, adoption of the assumption is a necessary and reasonable method for dealing with the uncertainties inherent in predicting the impact of a tolerance reduction. None of the comments suggested an alternative method for estimating the amount of fish that would be removed from commerce as a result of the proposed tolerance reduction.

Because of the comments it received questioning the justification for the proposed reduction in the fish tolerance, the agency has re-examined its projections of the food loss expected to result from such a reduction. The projections made in the preamble to the proposal were based on data obtained in 1974 on the levels of PCB's in commercial fish, primarily from the Great Lakes (Ref. 39), In making those original projections, the agency was forced to rely on the assumption that PCB levels in freshwater fish nationwide were as high as those found in the Great Lakes. FDA now has more recent and more representative data on PCB levels in commercial fish, which it obtained through a nationwide sampling program conducted in 1978 and 1979, Based on these more recent data, the value of the fish projected to be lost at tolerance levels of $5 \mathrm{ppm}$ and $2 \mathrm{ppm}$ is substantially less than was projected in the proposal. The loss projected under a $1 \mathrm{ppm}$ tolerance would remain about the same (Table B, "Regulatory Analysis for Final Regulation for Reduction of

Temporary Tolerances for Polychlorinated Biphenyls in Food," Ref. 46). Specifically, the amount of commercial fish now projected to be lost as a result of a 2 ppm tolerance is about $\$ 5.7$ million (expressed in 1974 dollars) compared to the previously estimated $\$ 8$ million; the current estimated loss of fish under a 5 ppm tolerance is about $\$ 0.6$ million (compared to the previously estimated \$1.1 million). Under a $1 \mathrm{ppm}$ tolerance, however, the projected fish loss, using the new sampling data on PCB levels, is about $\$ 16$ million (compared to the previously estimated $\$ 18$ million). The percentage of the freshwater fish catch now estimated to be lost under a 2 ppm tolerance is 14 percent (compared to the 25 percent that had been estimated from the 1974 data); under a $1 \mathrm{ppm}$ tolerance, the currently estimated loss of freshwater fish is 35 percent (compared to the previously estimated 43 percent) (Ref. 46),

As noted earlier in this preamble, many of the comments argued that the impact of the proposed tolerance reduction must be measured not only by the amount of the resulting fish loss but also by other economic impacts, such as potential unemployment and loss of income in the fishing industry and postulated disruption of the recreational fishing industry (e.g., reductions in boat, tackle, and bait sales]. The comments provided figures ranging into the hundreds of millions of dollars on the total economic value of these industries and, without offering any further analysis, contended that the impact on them would be "severe" or "major." The predicted impact on recreational fishing was premised on the possibility that State governments would severely curtail recreational fishing if the tolerance were reduced to $2 \mathrm{ppm}$.

In establishing a tolerance for PCB's in fish, FDA must take into account the amount of fish a given tolerance would remove from commerce. Section 406 of the act, however, neither requires nor authorizes FDA to weigh secondary economic impacts when it considers the level at which a tolerance should be set. Consideration of such impacts would be inconsistent with the paramount concern of section 406, which is protection of the public health, and would complicate the decisionmaking process under section 406 in a way 
Congress did not intend. Obviously, consideration of the amount of food loss caused by a tolerance helps to ensure that the direct economic consequences of the tolerance (in this case, decreased sales and employment in the commercial fishing industry) will not be disproportionate to the increased degree of public health protection accomplished by the tolerance; but the agency considers secondary economic consequences, such as potential impact on the recreational fishing industry, totally beyond the scope of section $\mathbf{4 0 6}$.

None of this should suggest that the agency is unaware of, or unconcerned about, the economic consequences of its actions. It is keenly aware that actions it takes to protect the public health can have adverse economic consequences, both direct and indirect, and that these consequences can sometimes be felt with particular severity in certain narrow segments of the economy. For example, some of the comments on the proposal argue that the impact of a 2 ppm PCB tolerance for fish will be especially severe for small-scale, freshwater fishers who specialize in certain species that happen to be heavily contaminated. The agency acknowledged this possibility in the preamble to the proposal (42 FR 17492).

in the present case, however, the agency has reason to believe that the claims of adverse economic impact are exaggerated. Based on the 1078/1979 data on PCB levels in freshwater fish, a $2 \mathrm{ppm}$ tolerance will remove from commerce about \$5.7 million worth of commercial fish. Although it is possible that fishing for certain heavily contaminated freshwater species may cease entirely in locations where PCB contamination is concentrated, at least some affected fishers-both commercial and sport-can be expected to adjust to the reduced tolerance by increasing their catch of other species or transferring their activities to other, less contaminated locations within their current area of operation.

In evaluating claims of economic impact, it is theoretically and pragmatically sound to take into account the motives and opportunities for adaptive behavior by affected individuals and fins. If the public demand for commercially caught fish remains stable or increases, and if the attractions of sport fishing remain strong, it can be expected that some fishing activity will shift to species that are not contaminated above the tolerance. Over time, the shifts will become easier as the levels of PCB contamination decline because more and more species will have average PCB levels well below $2 \mathrm{ppm}$. Over the long term, the adjustments will help to minimize the net economic impact of the tolerance reduction on both individual fishers and the overall commercial freshwater fishing industry.

None of the comments attempted to quantify in dollar terms the impact of the tolerance reduction on the recreational fishing industry, but several postulated a "severe" or "major" impact premised on voluntary decisions by individuals not to fish and mandatory curtailments of recreational fishing by State authorities. FDA is in no better position than were those submitting the comments to make precise predictions about the future behavior of individuals and State agencies. However, the agency considers the premises underlying the projections of "major" or "severe" impact to be somewhat speculative and of questionable validity. As noted to the extent that the behavior of individual recreational fishers is affected by the tolerance reduction at all, they, as much as commercial fishers, can be expected to adjust to the tolerance by shifting their activities to the less contaminated species and locations. Also, even if State agencies decide that some curtailment of recreational fishing is necessary in light of the reduced tolerance, it is reasonable to expect that their actions will be tailored by species and location. In the past, the most common response of State agencies to FDA's PCB tolerance for fish has not been the mandatory curtailment of recreational fishing. Instead, they have issued warnings concerning particular species and locations and made suggestions regarding both limitations on consumption of particular species and methods of preparing and cooking fish that minimize the amount of PCB's actually consumed from contaminated fish. Thus, there is little reason to believe that a $2 \mathrm{ppm}$ tolerance will lead to widespread, mandatory curtailment of recreational fishing and the resulting drastic economic impact the comments postulate.

\section{c. Conclusion}

Based on the data now before it, the agency concludes that a reduction of the fish tolerance from 5 to $2 \mathrm{ppm}$ will result in a meaningful decrease in the risk experienced by consumers from exposure to PCB's. Some reduction of the tolerance is clearly in order because the toxic effects associated with exposure to PCB's are serious and irreversible; and, due to declining levels of PCB contamination the current $5 \mathrm{ppm}$ tolerance permits contamination that can fairly be termed "avoidable" -even among the-more highly contaminated commercial species most likely to be affected by a reduced tolerance, only a minor percentage (about 1.5 percent) contain PCB's at levels as high as 5 ppm (Table A, Ref. 46). The agency's judgment is that the balance between public health protection and loss of food is properly struck by a $2 \mathrm{ppm}$ tolerance. As noted, as 2 ppm tolerance effects a meaningful decrease in risk to consumers while still excluding from commerce only a relatively small amount of food (about $\$ 5.7$ million landed value in 1974 dollars).

Several comments argued that an adequate degree of public health protection can be provided only by lowering the fish tolerance to $1 \mathrm{ppm}$, the lowest level at which PCB's can be reliably measured in fish for enforcement purposes. Indeed, as one would expect, the risk assessment performed by the agency, and discussed above, indicates that the estimated risks that might be experienced by consumers of contaminated fish would be reduced even further by a reduction of the tolerance to $1 \mathrm{ppm}$ (Tables 6 and 7, Ref. 45 ). Based on the evidence now before it, however, the agency does not consider a reduction to $1 \mathrm{ppm}$ necessary or appropriate in light of the policy of section 408 of the act.

The risk assessment the agency made incorporated several conservative assumptions that were designed to avoid understatement of the human risk. Thus, it is expected that the actual risk experienced by consumers of the 12 more heavily contaminated species covered by the risk assessment is less than that estimated. Moreover, the average consumer, who eats fish from a variety of freshwater and marine sources, will actually experience a far lower level of PCB exposure and a correspondingly lower degree of risk than those whose fish consumption is concentrated among the more heavily contaminated (predominantly freshwater) species. For these reasons, notwithstanding the quantified risk estimates produced by the risk assessment, the agency reaffirms the conclusion it expressed in the preamble to the proposal: The $2 \mathrm{ppm}$ tolerance provides an adequate degree of protection for all but those who consume above-average amounts of freshwater fish taken from contaminated waters (42 FR 17493).

In the agency's judgment, the additional increment of public health protection that might be provided by reducing the tolerance to $1 \mathrm{ppm}$ does not justify the additional loss of food that 
would resuIt. First, as discussed abov\&,CB's are forthcoming, the agency might the agency estimates that under a consider establishing a $1 \mathrm{ppm}$ tolerance tolerance of $1 \mathrm{ppm}$, approximately $\$ 16$ despite the effect that would have on the million worth of the commercial fish availability of food.

catch would be vioIative and thus,

For these reasons, the agency

presumably, removed from commerce.concludes that at this time a $1 \mathrm{ppm}$

This is nearly triple the $\$ 5.7$ million tolerance would not strike the proper

worth estimated to be violative under balance between protection of the

$2 \mathrm{ppm}$ tolerance. It is far more likely public health and the need to avoid under a $1 \mathrm{ppm}$ tolerance than under a excessive loss of food.

$\mathrm{ppm}$ tolerance that the more heavily Though FDA considers $2 \mathrm{ppm}$ to be contaminated species of freshwater fish the appropriate tolerance level for PCB' would be violative in percentages high enough to put an end to their commercial exploitation and, possibly, force some segments of the freshwater fishing industry to cease operations completely. Thus, the actual loss of food resulting from the $1 \mathrm{ppm}$ tolerance could greatly exceed even the $\$ 16$ million landed value (1974 dollars) estimated above.

Second, for the average consumer, current exposure to PCB's in fish is at a tolerably low level, when considered in light of the criteria of section 406 of the act, without a $1 \mathrm{ppm}$ tolerance, The average consumer eats a modest amount of fish from a variety of sources, both freshwater and marine, most of which yield fish with PCB levels below $1 \mathrm{ppm}$. Because their exposure is thus low to begin with, they are adequately protected by a $2 \mathrm{ppm}$ tolerance, which ensures that they will not be exposed to the unusually high levels of PCB's found in some species of fish. The slight additional protection these average consumers might gain from a $1 \mathrm{ppm}$ tolerance does not justify the

significantly greater impact such a tolerance would have on the availability of food. On the other hand, atypical heavy consumers (e.g., the Great Lakes sport fisher who catches and consumes large quantities of the contaminated species) would likely not be adequately protected by even a $1 \mathrm{ppm}$ tolerance because of the amount of fish they eat and because those fish are seldom affected by FDA tolerances (either because they are sport fish or are from intrastate commercial channels and, in either case, are outside FDA's jurisdiction). Protection of these consumers depends on actions by State authorities.

Finally, though the new toxicity data on PCB's clearly support the need to reduce exposure to this contaminant, the uncertainties in the data (discussed above) cast some doubt on the degree to which consumers are at risk from extremely low levels of PCB exposure, and therefore weigh against lowering the tolerance to $1 \mathrm{ppm}$. If, for example, more definitive and incriminating data on the reproductive risks posed by in fish under the criteria imposed by section $\mathbf{4 0 6}$ of the act, the agency is concerned about the health of certain groups that may not be adequately protected by a $2 \mathrm{ppm}$, or even a $1 \mathrm{ppm}$, tolerance. As noted, sport fishers and others who consume abnormally large amounts of the more highly from PCB's regardless of any tolerance FDA establishes. (The agency's risk assessment, using data from a study of Lake Michigan sport fish eaters, estimated that the upper limit on the lifetime risk of cancer for heavy eaters of sport fish from Lake Michigan is about 12 to 14 times greater than the corresponding risk for heavy eaters of those commercial fish most affected by a PCB tolerance, even assuming the tolerance remained at $5 \mathrm{ppm}$ (Table 6 , Ref. 45).] Those individuals, whose high exposures to PCB's tend to result from localized conditions and fishing practices beyond the control of FDA, should take steps to reduce their exposure to PCB's. FDA urges State and local health officials to evaluate the situation in their own localities and determine what steps, if any, they can take to address these special situations. In the past, some State and local agencies have made FDA's tolerance level for PCB' $s$ applicable to fish in intrastate commerce and have issued advisories to sport fishers warning that consumption of certain species of fish should be minimized and suggesting other ways in which PCB exposure could be reduced. These agencies should review their past actions in light of the current state of knowledge about PCB's and make the changes or take the additional steps that may now be appropriate, FDA will cooperate with these agencies, as it has in the past, by providing technical advice and assistance. FDA is sending letters to the governors of States most affected by PCB's in fish, discussing the agency's concerns about aspects of the PCB problem that may require an up-to-date review in their States.

The agency is advising that State health departments be particularly concerned about women of childbearing contaminated species may be at risk age, especially pregnant and lactating women, who may have consumed, or are consuming, higher than normal amounts of PCB-contaminated fish. Data that were discussed in the preamble to the proposal (42 FR 17468-9) suggest an association between PCB exposure and reproductive disjunction in rats and monkeys. They also show acute toxic effects in the nursing offspring of maternal monkeys that had been exposed to toxic levels of PCB's. Data gathered by FDA since it issued the proposal in 1977, and discussed in the report on FDA's risk assessment on PCB's (Ref. 45), establish more clearly the link between PCB exposure and adverse reproductive effects in the rhesus monkey. They also confirm the earlier data showing acute toxic effects in the nursing offspring of PCB-exposed maternal monkeys. As explained in the risk assessment report (Ref. 45), it is not possible at this time to determine with confidence the significance of these data in terms of human risk. There have been no reports of human reproductive abnormalities or overt toxic effects in nursing human infants that can be attributed to PCB's. That fact is of only limited significance, however, because epidemiological studies adequate to detect such adverse effects in humans have not been conducted.

An additional reason for concern in this area is that PCB's ingested by human mothers are found, and to some extent are concentrated, in human breast milk (see the discussions in the preamble to the proposal and in the risk assessment report (Ref. 45)). Ina recent nationwide survey, consisting of 1,038 samples of human breast milk collected in 44 States, the mean concentration of PCB's was estimated to be in the range of 1.00 to $1.10 \mathrm{ppm}$ (on a fat basis) (Ref. 45). Though the data are scanty, it is reasonable to assume that among women who consume above-average amounts of PCB-contaminated fish, or who are exposed to PCB's from other sources, the levels of PCB's in breast milk are significantly higher, As noted it is not now possible to determine the significance of these facts in terms of increased risk to the nursing infant.

In sum, although the agency concludes that a 2 ppm tolerance for PCB's adequately protects most consumers, women of childbearing age, especially pregnant and lactating women, are among those who should be careful to avoid abnormally high exposure to PCB's in fish, They can avoid such exposure by minimizing consumption of both commercial and noncommercial fish from waters known to be contaminated with PCB's and avoiding 
entirely those species of sport fish known to contain high levels of PCB's (e.g., coho and chinook salmon from the Great Lakes, and freshwater trout, striped bass, and catfish from some locations). State and local governments have the important role of advising consumers about conditions in particular localities.

The agency is aware that its decision to set the fish tolerance for PCB's at 2 $\mathrm{ppm}$, rather than leaving it at $5 \mathrm{ppm}$ or reducing it further to $1 \mathrm{ppm}$, is inherently judgmental in character. Section $\mathbf{4 0 6}$ of the act provides no formula for balancing public health protection against loss of food, and, hence, there is no way for the agency's decisions under section $\mathbf{4 0 6}$ to be arrived at mechanically or quantitatively or to appear clear-cut in every case. In this case, for example, forceful arguments have been made in the comments in support of both a $5 \mathrm{ppm}$ and a $1 \mathrm{ppm}$ tolerance, but those arguments all reflect the subjective" judgments of those who made them. In the end, the agency has been mandated by the Congress to make its own informed judgment about what is necessary to protect the public health. It has done that herein setting the fish tolerance at $2 \mathrm{ppm}$.

The statute provides an opportunity for a public hearing on the agency's order lowering the PCB tolerance for fish. Such a hearing would provide persons adversely affected by the order an opportunity to present any additional evidence they may have bearing on the matters that influenced the agency's judgment. As always, the agency is prepared to reevaluate its position in light of evidence adduced at a hearing.

\section{$D$. Other Comments on the Fish and Shellfish Tolerance}

In addition to the points addressed above, the comments raised several other points relating to the tolerance for fish and shellfish:

1. One comment recommended that FDA review its entire mechanism for handling recurrent problems of environmental contaminants in fish. The comment stated that the PCB tolerance should remain at $5 \mathrm{ppm}$ for marine fish because the levels in those fish are low enough that a reduction to $2 \mathrm{ppm}$ would have no increased protective effect, but would result in economic problems that are unnecessary for species with only occasional high PCB levels. The comment stated further that tolerances should be set for freshwater fish based on their individual place in the markettheir tonnage, distribution patterns, and consumption patterns. When such factors combine to present a risk, it was argued, the tolerance should be applied selectively to both the species and the body of water.

The individualized approach to establishing and enforcing tolerances for environmental contaminants suggested by this comment is not feasible because the necessary species-by-species, location-by-location data on PCB occurrence do not exist. Furthermore, many lots of fish, as currently packaged and shipped, do not bear the water-oforigin information required for the recommended regulatory approach. These limitations make it necessary for the agency to establish tolerances for fish on a generic basis. The result is a uniform regulatory approach for all species, which provides clear and fair rules for all segments of the fishing industry and is necessary to ensure that uncertainties and limitations in data will not result in increasing human exposure to PCB's. To the extent that certain species only occasionally have PCB levels above $2 \mathrm{ppm}$, the economic impact of the reduced tolerance will be slight.

2. One comment stated that any FDA regulatory action regarding PCB's in fish should apply to sport fish as well as commercial fish.

FDA's regulatory authority extends only to foods shipped in interstate commerce and clearly does not extend to fish caught and consumed by individual sport fishers. FDA cooperates with the State agencies who have authority over sport fishing by sharing data and views regarding toxicological, analytical, and compliance matters, but FDA has no direct control over the regulatory approaches adopted by the States. As noted, however, the agency urges State and local health officials to look closely at the PCB problem in their areas and take whatever steps they find necessary to address those aspects of the PCB problem, such as the exposure of sport fishers, that are beyond FDA's authority.

3. One comment requested reconsideration of the proposal to reduce the fish tolerance on the ground that overall ingestion of PCB's is reportedly declining. Because levels in other foods have already decreased considerably, it was argued, there is less need to lower the fish tolerance,

The agency is aware that PCB levels in foods other than fish have declined and that overall PCB intake is lower than it was in 1973, when the original temporary tolerances were established. However, as discussed in the preamble to the proposal and in section II of this preamble, toxicological considerations now make it desirable to reduce dietary exposure to PCB's even further. Reduction of the tolerance for PCB's in fish will bean especially effective step toward accomplishing that goal, because fish are the one remaining significant source of dietary exposure to PCB's.

4. One comment contended that fish products are being subjected to an entirely different regulatory standard than are poultry products, with no reasonable basis for the different treatment. The comment stated that the emphasis in establishing $2 \mathrm{ppm}$ as the tolerance for fish appears to have been safety to the consumer despite a considerable economic impact. Yet, it argued, the higher level of $3 \mathrm{ppm}$ for poultry is based on economic considerations relating to feed contamination, apparently without public health considerations. The comment went on to state that the average per capita consumption of fish is 19 grams $(\mathrm{g})$ per day compared to $63 \mathrm{~g}$ per day for poultry products. According to the comment, this means that under the proposed tolerances, and assuming maximum permissible levels in all foods, the average person will receive five times as much PCB's from poultry as from fish.

The agency does not agree with the comment's contention that the considerations involved in establishing the tolerances for poultry and fish result in different or conflicting regulatory approaches for these products, First, the $3 \mathrm{ppm}$ tolerance for poultry is based on PCB residues in the fat of the bird, not in all the edible tissue as it is for fish, Poultry generally averages about 10 percent fat; hence, the $3 \mathrm{ppm}$ tolerance is comparable to a level of about 0.3 $\mathrm{ppm}$ for the entire edible portion. Thus, even taking into account the higher average level of chicken consumption and assuming all foods contain maximum permissible amounts of PCB's, poultry will actually be regulated at a level that will result in a substantially lower intake of PCB's from poultry than from fish. Second, data show that detectable PCB residues occur so infrequently in poultry that exposure to PCB's from that source is already at an insignificant level. Hence, further reduction of that tolerance would not significantly reduce dietary exposure to PCB's and would not enhance protection of the public health. Fish data, on the other hand, show frequent occurrence of PCB residues at significant levels, so that reduction of the tolerance will result in increased protection for consumers of fish.

As explained in the preamble to the proposal (42 FR 17491-2), the agency selected 3 ppm (fat basis) as the 
tolerance for PCB's in poultry to allow for the regular use of poultry feed contaminated up to, but not exceeding, the $0.2 \mathrm{ppm}$ tolerance for PCB's in poultry feed. $(0.2 \mathrm{ppm}$ is the lowest feasible tolerance for PCB's in poultry feed because of limitations on analytical cap ability.) The $3 \mathrm{ppm}$ level took into account the biomagnification of PCB's in poultry that results from regular feeding with poultry feed contaminated up to, but not above, $0.2 \mathrm{ppm}$. The agency reasoned that it would be inconsistent to set tolerances on two products at levels such that the use of one product that complies with the applicable tolerance causes the second product to be illegal and, thus, that it would be inappropriate to do so in the absence of other overriding considerations [e.g., safety). For the reasons stated in the preceding paragraph, the $3 \mathrm{ppm}$ tolerance for poultry adequately protects the public health and is thus consistent as a matter of public health protection with FDA's other tolerances for PCB's.

5. One comment stated that any decision to lower the fish tolerance made in reliance on the regulation of point source discharges and manufacture of PCB's should not fail to consider the fact that PCB levels in contaminated waters are not expected to decline for many years.

The agency is aware that, despite Environmental Protection Agency (EPA) antipollution activities and the resulting gradual decline in PCB levels in at least some contaminated waters, there will continue to be a significant occurrence of PCB's in fish for at least the next several years because of the stability and persistence of the PCB's now contaminating the environment. That fact was taken into account in deciding to reduce the tolerances.

6. One comment stated that, because pollution of water with PCB's is expected to continue, PCB levels in fish will continue to rise, and susceptible fish should be harvested now before the increased contamination makes them all inedible.

Although the levels of PCB's in waters currently contaminated may not decrease substantially in the near future, the agency does not expect those levels to increase, nor does it expect the levels of PCB's in fish to increase. Better control of PCB levels should result from efforts by the EPA and industry to control discharge of additional PCB's into the environment. Hence, even if it were possible to harvest whole species of fish now, that step would not have the effect of preventing increased future exposure to PCB's. Finally, FDA has no authority to regulate the pace at which particular species of fish are exploited commercially.

7. Several comments stated that the decision to reduce the tolerance for fish should be reconsidered and the current 5 ppm level reaffirmed because PCB's are being steadily eliminated from the environment and may be expected to disappear as a significant problem within the next decade.

The agency does not agree that PCB's can be expected to be an insignificant problem within 10 years. Although EPA's continuing activities have resulted in a significant decrease in the amount of PCB's being introduced into the environment especially into water, the stability and persistence of these chemicals and the likelihood that some amount of additional contamination will continue to occur from waste disposal sites ensures that PCB contamination will remain a problem for the foreseeable future. Moreover, that PCB levels are declining (i.e., that PCB's are becoming more avoidable) is a reason to consider lowering the tolerance. not a justification for leaving it unchanged.

8. One comment argued that the decision to reduce the fish tolerance should be reconsidered because by lowering the fish tolerance, thereby preventing consumption of contaminated fish, some might be led to believe that the problem of exposure to PCB's had been solved. This misconception could in turn reduce the pressure to attack the real problempollution, However, the comment argued, if the environmental contamination itself is viewed as the "real" PCB problem of importance, changing the fish tolerance is almost irrelevant, given the small quantity of PCB's affected.

PCB contamination of the environment is itself an important part of the PCB problem because, as discussed in the preamble to the proposal (42 FR 17469-90), some human exposure to PCB's comes from the air and water, though the amount is probably minimal. EPA is addressing that part of the problem. However, FDA disagrees with the view that exposure to PCB's from dietary sources is insignificant in comparison to the amount of exposure from the air and water. The agency has based the proposed tolerance reductions on its conclusion that dietary exposures to PCB's pose significant risks to consumers, which can be reduced by reducing exposure. That there is some exposure to PCB's from other sources is not a good reason for withholding action that can significantly reduce dietary exposure.

9. Two comments requested FDA to hold a public hearing before finalizing reduction of the fish tolerance.

The agency does not consider a public hearing on the fish tolerance to be necessary or appropriate at this time. Tolerances are established under section 406 of the act under the formal rulemaking procedures set forth in section 701(e) of the act (21 U.S.C. 371(e)]. Under those procedures, any person adversely affected by this order may file objection within 30 days and request an evidentiary hearing on the issues raised by those objections. The opportunity for a hearing ensures that all genuine, material issues relating to the PCB tolerances will be fully aired.

Holding a hearing before issuing this order would only duplicate the opportunity for a public hearing already available in formal rulemaking and unnecessarily delay the proceedings.

10. One comment stated that the 5 ppm tolerance for PCB's in fish should be retained but requested that FDA provide guidance to State agencies regarding use or implementation of the 2 $\mathrm{ppm}$ tolerance if it is adopted.

As noted, FDA provides data and views to the States on a range of matters related to implementation of tolerances for PCB's in food and will continue to do so.

11. One comment asked whether procedures other than reducing the fish tolerance have been evaluated as alternative means of reducing intake of PCB-contaminated fish.

The agency has considered the use of general public warnings and/or labeling as ways to limit consumption of contaminated fish. Such approaches have been rejected, except as they apply to certain heavy consumers of contaminated sport fish (discussed above]. A public warning about fish generally, or even about particular species of fish, would not be effective in protecting the general public from commercial fish because, assuming no changes are made in labeling, consumers have no way to determine the species or waters of origin of most commercially prepared fish products. In addition, general public warnings might unduly discourage consumption of fish, most of which is safe to eat and nutritious. Similarly, the requirement of warning labels on fish products in lieu of a tolerance, even on a species-specific basis, is not a sufficientl precise $_{\mathrm{y}}$ regulatory approach because not all fish from even the most heavily contaminated species contain levels of PCB's above the 2 ppm tolerance level. 
Thus, as with general public warnings, warning statements on labels are likely to discourage consumption of safe fish.

12. Some comments contended that most Americans would probably prefer to be warned of the potential danger from PCB residues and retain the option of eating freshwater fish, rather than be deprived of any choice in the matter by having the fish removed from the market.

The agency acknowledges that some people would probably prefer to be left with the choice of whether to consume fish contaminated with PCB's above the $2 \mathrm{ppm}$ level. As noted, however, the consumer of commercially marketed fish generally lacks the information on water-of-origin, size, and sometimes even species that is needed to control his or her intake of PCB's. Under these circumstances, there is no genuine opportunity to exercise informed choice. Moreover, the agency believes that as a general matter it is obligated under section $\mathbf{4 0 6}$ of the act to exercise its scientific judgment and determine what level of exposure, and thus what tolerance level, will provide an adequate degree of public health protection,

13. One comment referred to the agency's decision not to reduce the temporary tolerances for infant and junior foods and for animal feeds on the ground that the current tolerances are "at the lowest level at which PCB's can be reliably determined for enforcement purposes" and argued that lowering the tolerance for fish would probably create much greater economic hardship than would developing and using more sensitive analytical methods so that other tolerances could be lowered,

The agency acknowledges that the fish losses resulting from a $2 \mathrm{ppm}$ fish tolerance would probably be greater than the costs of developing and using the more sensitive enforcement analyses that would be necessary for a reduction of the other tolerances. The occurrence of PCB residues in infant and junior foods and animal feeds is now so infrequent, however, that those foods do not contribute significantly to dietary exposure to PCB's. Thus, spending the resources to develop more sensitive methodology and thereafter reducing the tolerances for these foods would not significantly increase the protection of consumers, and it still would be necessary to reduce the fish tolerance, Because PCB's do occur consistently at significant levels in some fish, the reduction of the fish tolerance can provide increased protection for consumers.
14. Some comments included requests for compensation for commercial fishers and processors whose livelihoods are destroyed by reduction of the fish tolerance. One comment asked, in effect, that the effective date of the tolerance reduction be delayed for 10 years so fishers would have time to adjust economically.

For reasons discussed earlier in this document, the agency considers it unlikely that the reduction of the fish tolerance to $2 \mathrm{ppm}$ will have the dire consequences on which these comments are premised. Moreover, FDA has neither the authority nor the resources to provide compensation for economic losses that might be suffered as a result of regulatory actions it takes. The proposed 10-year postponement of the effective date would be inconsistent with the agency's conclusion that a reduction of the tolerance is necessary to protect the public health.

15. A number of comments were concerned that if the $2 \mathrm{ppm}$ tolerance for fish is adopted, FDA will close certain waters to fishing or prohibit fishing of certain affected species in certain waters. They requested that more studies be carried out before determining whether such steps should be taken.

The concern underlying these comments is misdirected, FDA does not have authority either to close waters to fishing or to prohibit harvesting or possession of fish. Any actions to close waters to fishing would have to be instituted by State agencies.

16. One comment suggested that if the $2 \mathrm{ppm}$ tolerance is adopted, the counties affected should be allowed to conduct more comprehensive testing of residue levels in the fish before any ban or impoundment of fish in interstate commerce is imposed.

In enforcing the fish tolerance, FDA will sample and analyze individual lots of fish in interstate commerce and take regulatory action against lots, or the shippers of lots, that exceed the tolerance, There is nothing to prohibit any interested party, including local and State authorities, from conducting comprehensive testing of fish before shipment in interstate commerce and from withholding from commerce fish that exceed the tolerance.

17 , One comment suggested that the proposed $2 \mathrm{ppm}$ tolerance for PCB's in fish is inadequate for protection of public health, The comment stated that the tolerance levels must be based on the "no-effect" level observed in-the most sensitive animal species for which toxicological data are available, and it suggested that the rhesus monkey is more sensitive to PCB's than the dog or rat.

This comment is based on an apparent misunderstanding of the toxicological rationale underlying the 2 ppm fish tolerance. In evaluating the safety of substances in food, FDA ordinarily attempts to determine the "no-effect" level for the substance, i.e., the highest level of exposure at which no adverse effect is observed in appropriate animal studies, It then uses appropriate safety factors to extrapolate the results of the animal studies to the human situation and determine safe levels of human exposure. In this case, however, the reduction of the fish tolerance is not based on any "noaffect" level, It is based instead on a body of data that associate PCB exposure with several serious chronic effects but that do not permit the establishment of "no-effect" levels for those effects, Thus, the comment's argument that one species is more sensitive to PCB's than another and that the tolerance should be based on the "no-effect" level observed in the most sensitive animal species is not relevant to the toxicological rationale the agency relies on for reducing the PCB tolerance to $2 \mathrm{ppm}$.

18. One comment disagreed with the proposal to establish a $2 \mathrm{ppm}$ tolerance for fish instead of a $1 \mathrm{ppm}$ tolerance. One ppm is the lowest level of PCB residues in fish for which there is analytical methodology suitable for enforcement purposes. The comment stated that toxicological information, especially that suggesting the carcinogenicity if PCB's coupled with the presence of PCB residues in human milk, requires the lowest possible tolerance.

With respect to the carcinogenic potential of PCB's, NCI has concluded that PCB's (specifically, Aroclor 1254, the commercial PCB most similar chemically to the PCB residues in fish) are not carcinogenic in Fischer 344 rats under the conditions of the bioassay (Ref. 47). After thoroughly reviewing NCI's report, the Data Evaluation/Risk Assessment Subgroup of the Clearinghouse on Environmental Carcinogens accepted the report's conclusion that PCB's were not demonstrated to be carcinogenic in that study, but suggested that PCB's might act as a tumor promoter. For the reasons discussed in the preamble to the proposal (42 FR 17489), FDA considers the question of the carcinogenicity of the PCB's unresolved. For the purposes of its risk assessment on PCB's (Ref. 44), however, the agency treated the various PCB's as though they were carcinogenic, 
and it considers the carcinogenicity of PCB's to be a matter worthy of further serious inquiry.

The agency has long been concerned with the exposure of nursing infants to PCB's in human breast milk. This, too, is an area in which more must be learned before definitive statements can be made about the incremental risks posed by this particular avenue of exposure. For reasons discussed earlier in this document, however, the agency considers a $2 \mathrm{ppm}$ tolerance adequate to protect all but those who consume above-average amounts of the more heavily contaminated species.

111. Response to Comments on Other Aspects of PCB's

Following are the agency's responses to the comments that did not specifically address the reduction of the fish tolerance:

1. Some comments recommended that the government regulate PCB's only in the environment rather than in food products. Another comment suggested that the limits on PCB's in foods not be reduced until PCB levels have been reduced in the environment, where the foods are produced.

EPA has the authority to control environmental pollution and has already taken important steps to prevent further pollution by PCB's. Some environmental contamination with PCB's already exists, however, and will undoubtedly persist for some years. FDA would be failing in its duty to protect the public health if it withheld the actions necessary to minimize human exposure to PCB's from dietary sources until the long-term problem of environmental contamination has been solved.

2. One comment asserted that the primary toxicological basis upon which FDA established the temporary tolerances for PCB's in 1973 (38 FR 18096) consisted of two long-terms feeding studies in rats and dogs that were performed by the same testing laboratory and that demonstrated a "noeffect" level for PCB's at $10 \mathrm{ppm}$. This comment also suggested that these same two studies serve as the primary basis for the current proposal to reduce those original temporary tolerances: The comment stated that discrepancies and inconsistencies have recently been found in these two feeding studies, as well as in other unrelated studies from the same testing laboratory, which would indicate that toxic effects might actually have been produced in both rats and dogs, at dietary levels as low as $1 \mathrm{ppm}$. The comment requests that FDA extend its audit of the testing laboratory in question to include a review of the data obtained in the two toxicity tests of PCB's in rats and dogs. The comment suggested that FDA reconsider the proposed temporary tolerances on the basis of a reevaluation of the data from the two long-term studies and, if judged necessary, propose new tolerances or reopen the matter for public comment.

Though the data from the two longterm toxicity studies of PCB's in rats and dogs referred to in the comment were considered, the human toxicological data formed the primary basis for developing the original temporary tolerances for PCB's. This fact was stated in the July 6, 1973 document establishing the tolerances and in the preamble to the April 1,1977 proposal to reduce some of the tolerances.

The agency is aware that doubt has been cast on the validity of the two long-term toxicity tests of PCB's in rats and dogs referred to in the comment, as well as on the validity of numerous unrelated toxicity tests performed by the laboratory facility in question (Ref. 48). Therefore, the results from these two tests are no longer considered worthy of reliance and, as explained earlier in this document, these studies played no part in the agency's decision to lower the PCB tolerances.

3. One comment asserted that the agency's statement in the preamble to the proposal that it was unaware of any consumers who had suffered deleterious effects caused by PCB ingestion (42 FR 17491 ) is misleading, in that the statement actually reflects a lack of knowledge rather than awareness of the results of properly designed epidemiological studies.

In making this statement the agency relied on, the results of an epidemiological study carried out with sport fishers in Michigan that failed to establish a correlation in humans between the ingestion of PCB's and the occurrence of deleterious effects (Ref. 40). The study is discussed in the preamble to the proposal (42 FR 17492$3)$. The only purpose of the statement was to cite an instance in which relatively high exposure to PCB's in fish had not resulted in overt, acute toxic effects, such as occurred in the Yusho incident in Japan (42 FR 17488). The Michigan example was intended to illustrate the observation the agency made in the preamble to the proposal that the amount of PCB's in environmental samples required to cause Yusho-type effects is not known. This study has no direct bearing on the agency's conclusion that the chronic effects of PCB's require a reduction of the tolerances,
4. One comment opposed reduction of the temporary tolerance for PCB's in eggs on the ground that there are no substantial data that suggest that the current temporary tolerance is not sufficient to protect consumers of eggs. The comment contends that lacking such evidence, there is not justification for reducing the tolerance.

The agency acknowledges that the data indicate that eggs do not contribute measurably to dietary PCB exposure and that reduction of the egg tolerance will not significantly affect PCB intakes. However, tolerances established under section $\mathbf{4 0 6}$ of the act are intended to permit only those residues that are unavoidable. Because the available data indicate that residues above the analytical limits in eggs are avoidable and because no evidence was presented to the contrary, it is appropriate to reduce the temporary tolerance for PCB's in eggs as proposed.

5. One comment requested that the temporary tolerances for PCB's in animal feed be reduced, but it did not present a rationale to justify reduction.

The presence of PCB's in animal feed is of concern because PCB's transfer and accumulate in human food products derived from animals that consume contaminated feed. The tolerance for finished animal feed is currently set at $0.2 \mathrm{ppm}$ - the lowest level at which available analytical methodology can measure PCB's in animal feed for enforcement purposes. It would serve no useful purpose to reduce the tolerance below this level in the absence of analytical methodology for enforcing a reduced tolerance. Moreover, in light of the rare occurrence of PCB's in animal feeds, the agency considers the $0.2 \mathrm{ppm}$ level to provide an adequate degree of public health protection, For these reasons, the agency declines to reduce the tolerance for PCB's in finished animal feed.

IV. "Temporary" Status of the Tolerances

As currently codified in 109.30 , the tolerances for PCB's are designated as "temporary," The term "temporary" was used to reflect the fact that the tolerances are subject to revision as new data become available. In the preamble to the proposal for reducing the tolerances, the agency stated that it would retain the "temporary" designation because of the possibility that further downward revisions of the tolerances might be necessary (42 FR 17493). The agency has now reconsidered this use of the term "temporary" and has decided to abandon it. The term has never had any 
legal significance as applied to tolerances established under section 406 of the act, and its use is not provided for in FDA's procedural regulations governing tolerance setting in Part 109 (21 CFR Part 109). When circumstances are changing so rapidly that a particular tolerance level is likely to be rendered inappropriate in the near future, the agency establishes an action level rather than a tolerance (see $\$ 109.6(\mathrm{c})$ ) (21 CFR 109.6(c))). In the case of PCB's, however, the agency has concluded that formal tolerances are appropriate. The term "temporary" is being abandoned to avoid the suggestion that the legal status of the PCB tolerances is something other than that of a formal section 406 tolerance.

Any FDA tolerance, just like any other regulation, is "temporary" in the sense that it is subject to reevaluation and, if necessary, revision as new data become available. The agency will continue to monitor the PCB problem and, if appropriate in light of changing circumstances or new data, will propose revisions in the PCB tolerances.

\section{Analytical Methodology}

Section 109.30(b) has been revised to refer to FDA's updated compilation of analytical methodology for PCB's "Analytical Methodology for Polychlorinated Biphenyls, June 1979." There have been improvements in the analytical methodology for measuring PCB residues since 1973, and most of the revised procedures have now been published in scientific journals. A copy of each procedure or a reference to the appropriate journal is provided in the updated compilation. As stated in $\$ 109.30$ (b), the compilation is available from the Hearing Clerk, Food and Drug Administration, Room 4-65, 5600 Fishers Lane, Rockville, MD 20857.

\section{References}

The preamble to the proposal cited 42 references the agency relied on in developing the proposal and stated that those reference documents had been placed on file with the Hearing Clerk, FDA (42 FR 17493-4). The following additional references, which are cited in the foregoing preamble, have also been placed on file with the Hearing Clerk, FDA, Rm. 4-85, 5800 Fishers Lane, Rockville, MD 20857, and may be seen between 9 a.m. and 4 p.m., Monday through Friday.

43.DuBois, K. P. and E. M. K. Ceiling,

"Textbook of Toxicology," Oxford

University Press, 1959, pp. 24-28.

44. Ariens, E. J., A. M. Simonis, and J.

Offermeier, '*Introduction to General
Toxicology,” Academic Press, 1976, pp. 124-31.

45. An Assessment of Risk Associated with the Human Consumption of Some Species of Fish Contaminated with Polychlorinated Biphenyls(PCB's), 1979, FDA document.

46. Regulatory Analysis for Final Regulation

for Reduction of Temporary Tolerances for

Polychlorinated Biphenyls in Food, 1979

FDA document,

47. National Cancer Institute Carcinogenesis

Technical Report Series No. 38, 1978.

48. Letter from Donald Kennedy,

Commissioner of Food and Drugs, to

various clients of Industrial Bio-Test

Laboratories, Inc., June 1977.

Therefore, under the Federal Food,

Drug, and Cosmetic Act (sees. 306, 402(a), 406, 701(a), 701(e), 52 Stat. 10451046 as amended, 1049 as amended, 1055, 70 Stat. 919 as amended (21 U.S.C. 336, 342(a), 346, 371(a), 371(e))) and under authority delegated to the

Commissioner of Food and Drugs (21

CFR 5,1), Part 109 is amended in $\$ 109,30$ by revising the section heading and paragraphs (a)(1), (2), (3), (4), and (7] and (b) to read as follows:

$\$ 109.30$ Tolerances for polychlorinated biphenyls (PCS'S).

(a) $\bullet \bullet$

(1) 1.5 parts per million in milk (fat basis).

(2) 1.5 parts per million in

manufactured dairy products [fat basis).

(3) 3 parts per million in poultry (fat basis).

$$
\text { (4) } 0.3 \text { part per million in eggs. }
$$

(7) 2 parts per million in fish and shellfish (edible portion), The edible portion of fish excludes head, scales, viscera; and inedible bones.

(b) For determining compliance with the tolerances established in this section, a compilation entitled "Analytical Methodology for Polychlorinated Biphenyls, June 1979" is available from the Hearing Clerk, Food and Drug Administration, Department of Health, Education, and Welfare, Room 1-65, 5600 Fishers Lane, Rockville, MD 20857 ,

Any person who will be adversely affected by the foregoing regulation may at any time on or before July 30, 1979, submit to the Hearing Clerk (HFA-305), Food and Drug Administration, Rm. 465, 5600 Fishers Lane, Rockville, MD 20857, written objections thereto and may make a written request for a public hearing on the stated objections. Each objection shall be separately numbered and each numbered objection shall specify with particularity the provision of the regulation to which objection is made. Each numbered objection on which a hearing is requested shall specifically so state; failure to request a hearing for any particular objection shall constitute a waiver of the right to a hearing on that objection. Each numbered objection for which a hearing is requested shall include a detailed description and analysis of the specific factual information intended to be presented in support of the objection in the event that a hearing is held; failure to include such a description and analysis for any particular objection shall constitute a waiver of the right to a hearing on the objection. Four copies of all documents shall be submitted and shall be identified with the Hearing Clerk docket number found in brackets in the heading of this regulation,

Received objections may be seen in the above office between the hours of 9 a.m. and 4 p.m., Monday through Friday.

Effective date. This regulation will become effective for foods initially introduced into interstate commerce after August 28, 1979 except as to any provisions that may be stayed by the filing of proper objections. Notice of the filing of objections or lack thereof will be announced in the Federal Register,

[Sees. 306, 402(a), 408, 701(a), 701(e), 52 Stall 1045-1046 as amended, 1049 as amended, 1055, 70 Stat. 919 (21 U.S.C. 336, 342(a), 346, 371(a], 371(e)])

Dated: June 26, 1979.

Donald Kennedy,

Commissioner of Food and Drugs.

[FR Dec. 79-20266 Filed 6-29-79; 8:45 am]

BILLING COOE 411 O-O3-M 


\section{Methods for Toxicologic Testing *}

by SRI International

\section{METHODS FOR TOXICOLOGIC TESTING}

In evaluating substances for their toxicologic effects. several essentials must be a part of each test protocol so that the toxicologic profile is as accurate as possible within the limitations of each test. This is true whether the test material is used for in vitro assays or administered in vivo by inhalation, gavage, dietary, dermal, subcutaneous, intravenous. or any other route to experimental animals. Both the testing and subsequent evaluation of data must be done under the direction of individuals having the necessarveducation, training, and experience to conduct the testing and interpretation of results in accordance with sound scientific principles and good laboratory practices. These principles and practices have been addressed and elaborated on in the FDA proposed guidelines (1) for " Good Laboratory Practices in Non-Clinical Laboratory Studies." It is important to realize that, at present, extrapolations from animal test systems to humans to predict effects of exposure to hazardous materials can be made only tentatively.

The data used for the evaluation of potential risk should be derived from tests conducted with the form of the toxicant that poses the health hazard. In some cases, this will be the lechnical grade of the active ingredient. In other cases, it could be the manufactured end product, the pure grade of the material, an inert ingredient of the lest substance, or a metabolite or degradation product of the substance so that the study can be completed using the same lot sample. Within the limits of analytical detectability, the chemical composition of the test material must be determined. If the test substance is to be mixed with the diet or another vehicle, the concentration and homogeneity must be ascertained prior to beginning the study. During the course of the investigation, random samples should be taken to ensure

*Excerpt from OTA Working Paper entitled "Assessment of Methods for Regulating 'Lnavoidable' Contaminants in the Food Supply." A romplete copy of the paper can be obtained from the National Technical Information Service. (See app. J.) that the required concentration of the substance is maintained.

Healthy animals must be used and maintained under conditions conforming to good husbandry practices (1). Animals must be assigned to test groups in a way that minimizes bias and assures that the number of animals in each dose group and the number of dose groups are sufficient to yield statistically valid results. The animals used in the study should be of uniform weight and age and should be an adequate representation of the sex, species, and strain under consideration. In addition to the treated groups, negative control groups (both historic and concurrent) meeting the above specifications must also be used to evaluate any toxic manifestations that may have occurred independent of the test substance. In cerlain situations, positive control groups may be required. This is particularly necessary for many short-term in vitro assays. Concurrent controls must be handled in the same way as the treated animals so that the treated and control groups will be validly comparable. Control groups take on added importance if a carrier is used in administering the test material to confirm that the carrier selected for use is nontoxic, induces no independent physiological effects, and does not change the chemical characteristics or toxicity of the test material.

Toxicology testing methods can be categorized by duration and endpoint. Short-term tests are usually considered to require 90 days or less for data gathering. These may include simple tests. such as 2-hour LD50 range finding, or more complex ones, such as 90-day continuous exposure or paired feeding studies. Tests requiring more than 90 days, such as lifetime exposure studies, are generally considered long-term tests. In addition to the time necessary for exposure and data gathering, analysis of the results may take up to an additional year depending on the complexity of the experiment, the number of animals used in the experiment, and the volume of data collected. Methods may also be categorized by endpoint. In this 
scheme, experiments are designed and data are collected based on expected results such as functional systemic changes, teratogenicity, or carcinogenicity. By the use of an appropriate experimental design, several endpoints can be assessed in the same experimental period such as is done in FDA's three-generation studies (2).

For ease of presentation, this appendix has been subdivided by endpoint into sections on Systemic Toxicity, Carcinogenicity, Mutagenicity, Teratology and Effects on Reproduction, Metabolism, and Structure-Activity Relationships. This appendix is not intended as an exhaustive survey of all testing methods used, but is meant to give an overview of those methods most commonly used today by toxicologists.

\section{Local and Systemic Toxicity}

Some of the fastest and simplest methods for determining the toxicities of substances involve the observation of changes in the structure and function of organs and organ systems. These methods generally involve absolute and relative weight changes, gross and microscopic structural alterations, and primary and secondary tests for organ, system, or whole animal function. With advances in the chemical, physiological, and behavioral sciences, modifications for testing systemic toxicity have been proposed that make these procedures more sophisticated and relatively complicated. Several good texts are available which review systemic toxicity $[3,4)$.

\section{Range Finding}

The classic determinations of toxicity involve percent lethal or effective dose, concentration, or time. These tests may employ any route of exposure, the ones chosen usually being based on factors such as chemical and physical properties of the agent and potential routes of exposure from the environment. The results obtained from these determinations are usually specific for the species, sex, age, and condition of the organism, and for the route of exposure and environmental conditions before, during, and after exposure. The endpoints of these tests may be either structural or functional changes, but they are usually limited to gross effects such as death or narcosis. These tests are primarily used to determine relative toxicities of various agents and for range finding for maximum tolerated dosage preliminary to beginning a subacute study. They are not usually used to directly evaluate the hazard.

In general, these tests will employ young adult rats and another mammalian nonrodent species.
Selection of this other species " . . should consider such factors as comparative metabolism of the chemical and species sensitivity to the toxic effects of the test substance ...."(1) The route of administration chosen is that most nearly identical to the potential human exposure. Doses are usually chosen to give results in the 20- to 80percent lethal or effective range and are usually separated by $0.5 \mathrm{log}$ units (5). Many modifications of this basic procedure are accepted.

\section{Irritation}

The irritation potential of substances is tested by observation of the reflex behavior of the animal and by direct observation of the site of contact with the agent, Attempts to quantitate reflex behavioral responses, i.e., eye rubbing, regurgitation, or shallow breathing, have met with little success. The simplest protocols for evaluating irritation involve the skin and eyes. Semiquantitative systems for scoring skin and eye irritation have been proposed by several authors (6-12) and involve placing the suspected irritant in contact with the skin or eye of New Zealand White rabbits. Protocols for skin irritation involve contact with both intact and abraded skin to differentiate the agent's ability to penetrate the skin barrier, and occlusion of the contact site to maximize the response. The severity of erythema and edema is scored as the endpoint. Eye irritation studies are carried out without washing and with washing at various intervals to determine the effectiveness of removal of the agent to reducing the adverse effect, Opacity, area affected, iris reaction to light, hemorrhage, swelling, and discharge are scored. Results from these tests can vary greatly depending on the method of application of the substance, whether dry or premoistened, etc.

Other potential sites of irritation such as the sensory nerves, respiratory system, urinary system, and gastrointestinal tract are usually evaluated secondarily or through necropsy. Secondary effects include shallow breathing, regurgitation, agitation on urination, and eye rubbing, which are broadly categorized as reflex behavior, and blood in feces, urine, and sputum, or nasal discharge, which are more indicative of the primary irritant effect. These results are not quantifiable by present methodologies. Primary evaluation through necropsy is also a qualitative procedure and has not undergone the extent of standardization and validation that the skin and eye tests have. Other methods for testing irritation such as resistance/compliance tests of the pulmonary system, direct observation by scope of the esophagus 
and gastrointestinal tract, and roentgenographic examination with and without radio-opaque dyes, have not received wide use as testing techniques for Government regulatory purposes.

\section{Sensitization}

Some substances, although not necessarily primary irritants, elicit an irritant-type response after repeated contact with the organism. Tests for this sensitization potential involve exposing the animal to an agent at doses below those necessary to produce signs of primary irritation, waiting an appropriate interval, and then challenging the animal with the substance again at a different site (11). If the response on retest is substantially higher than the initial response, the agent can be classified as a sensitizer. Various test methods and modifications have been proposed for testing sensitizing potential $(11,13-21)$. Perhaps the most common direct test for sensitization is the guinea pig maximization test. In this test, the agent is presented in Freund's complete adjuvant which increases the response. Studies on the mechanism of sensitization have shown that the agent or a metabolize of it (antigen) may induce the lymphocytes of the body to form a complex molecule (antibody) which reacts with the antigen to form an antigen-antibody complex. This reaction may be with circulating free antibody or with lymphocyte-bound antibody, The formation of the antigen-antibody complex induces the production and release of histamine and other compounds which cause the erythema and edema at the site of antigen attack, or may cause anaphylaxis if the antigen reaches the blood stream $(22,23)$. The problem with testing methods based on this mechanism, such as immunoelectrophoresis, radioimmunoassay, ring test, hemagglutination tests, or microphage migration, is that they do not measure the actual adverse effect [dermatitis or shock) but measure an indicator response. The methods are valuable, however, in demonstrating the presence of antibody capable of producing these health effects. Several comparative tests have demonstrated that these in vitro techniques are often more sensitive indicators of the hazard than the classic in vivo ones $(22,23)$.

\section{Structural Effects}

The basic determination of structural effects on organs and systems begins with the determination of absolute and relative weight changes. Decreases in absolute body weight or rates of weight gain for a test with a substance incorporated into the food or water may show either that the agent is unpalatable or that it is interfering with the energy balance, the central nervous system (CNS) regulation of food or water consumption, or the motivation of the animal. Although substances administered by other routes of exposure may also interfere with palatability of food, through direct or indirect effects on the sensory nerves, this is less common. Increases in weight may be caused by a proliferating tumor mass. The evaluations of structural changes can be obtained from animals exposed during subacute experiments.

In general, subchronic or subacute experiments are designed to last approximately 10 percent of the animals' lifespan (90 days for rats). Immediately preceding and during the experimental period, observations on animals should include rate of growth, food and water consumption, demeanor, and reflex behavior; blood, urine, and feces should be collected. During the experimental period, tissue biopsies may be taken for observation of structural changes. These techniques may be unreliable, however, if a structural change is localized and not included in the biopsy material, and such manipulation is often not allowed by regulatory testing guidelines. If biopsies are done, additional animals are required to maintain the statistical validity of the experiment. At the end of the experimental period, the animals are sacrificed and the organs are inspected for gross changes, removed and weighed, and preserved for histologic treatment and microscopic examination (5). The specific organs and tissues removed and examined will depend somewhat on the expected action of the agent administered (usually perceived from preliminary testing) but should include at least the brain, liver, kidneys, spleen, heart, testes (and epididymis) or ovaries (and uterus), thyroid, and adrenals.

Changes in organ weights may signal a functional change in this organ or in other organs; for example, an increase in heart weight could be due to a decrease in oxygen diffusion from the lungs, an increase in adrenal weight could signal a blockage of steroid synthesis within it, etc. The weights of organs can be directly compared with those from control animals; however, this often introduces an artifact since experimental and control body weights are usually different. It is common practice therefore to determine the relative weights of the organs in relation to the total body weight of the animal. Recently it has been proposed that the relative weights should be taken as a function of the animal's brain weight, the postulation being that the brain's growth curve devi- 
ates the least of any tissue in the body. While normalization based on this procedure would tend to emphasize changes more than other techniques currently in use, it is not yet widely accepted. Tissue dry weight, after desiccation or ashing, has also been used as a tool for determining mechanisms of growth and metabolic balances (24). This method has a major drawback, however, because it removes the organ from further studies such as microscopic examination.

After gross observation and weighing, the organs and tissues are preserved for histological preparation and microscopic examination. The most common methods for the preparation and staining of individual tissues involve fixing with 10-percent buffered formalin solution, embedding in parafin, and staining with hemotoxylin/eosin, Many pathologists prefer other fixing and embedding media, and certain tissues require different procedures. There are also special stains for highlighting different cellular components. There is no one best method for preparation and observation of the tissues. The most valuable procedure from the pathologists' viewpoint is to prepare the tissues in a number of ways, which allows comparison of various aspects such as specific cellular components, nuclei, cell membranes, etc. (25-27).

Special consideration can also be given to techniques in histochemistry and electron microscopy. These methods are not used routinely in toxicological evaluation and depend on a knowledge of the mode of action of the toxic agent. They can, however, indicate changes in cellular metabolism or structure before those changes become manifest by the conventional histological procedures, and therefore they may be more suitable for observing changes from agents whose toxicities are low or develop slowly. The equipment necessary for these techniques is generally more expensive than that needed for the more conventional microtechnique methods. They are also more time consuming and less standardized than conventional methods. The histochemical methods, although they might be more appropriately classified as tests of organ function, are becoming more widely accepted with investigators studying mechanisms of toxic: action.

\section{Functional Effects}

Frequently, changes in organ or system function are observable before any change in structure becomes apparent. The test methods discussed below have generally been adapted from human to animal use, and results are ordinarily compared with animal control values and are not necessarily comparable between species, Methods for evaluation of pulmonary function (28), cardiovascular function (29), and brain and neural activity (30) have been modified for human and animal use. These methods include testing ventilator flow, resistance, compliance, and gas diffusion capacity for the pulmonary system; electrical activity of the heart, and blood flow and pressure for the cardiovascular system; electrical activity of the brain (field and single unit) and muscles; perception threshold, reflexes, and chronaxy for neural function. The significance of changes in brain activity as a determinant of toxicity is under question at present, however.

Generally, in these evaluations, each animal serves as its own control. Baseline data for each procedure is determined prior to administration of the agent, and any changes in function are noted during and after administration, since it is important to determine whether the agent causes reversible or irreversible changes in function.

Various other methods of testing for organ or system function rely on both primary and secondary parameters. For example, liver function may be assessed by dye clearance studies (primary) or by analysis of serum enzyme concentrations (secondary). Most of the secondary procedures are now automated and available through various clinical laboratories at a reasonable cost, Many investigators, however, still prefer to perform the tests manually, and standard procedures are well-defined and available in several texts (31-33). Various modifications of these tests for specific animal systems have been developed and published, Tests usually considered appropriate include total and differential blood counts, serum enzyme and ion analysis, urinalysis (especially for metabolizes of the agent), and liver and kidney function tests (dye clearance). The value of these tests is that abnormal results will often precede obvious structural damage of the organ system in question and will be apparent at lower dose levels.

The study of hematologic effects encompasses changes in the bone marrow as well as those in the cells of the circulating blood. Observations are made of the cells and of their absolute and relative numbers. Specific tests such as dye dilution for blood volume, specific gravity, sedimentation rates, osmotic fragility, hematocrits, or clotting time, are not routinely performed but may be indicated. Serial bone marrow biopsies may also be performed for hematologic effects; the results 
give both structural and functional information, but these techniques are also not widely used.

The functions of the liver may be tested for biliary obstruction (icterus index, alkaline phosphatase), liver damage (thymol turbidity, plasma protein ratios, cholesterol ratio, glucose level, transaminase level, and cholinesterase level), excretory function (bromsulphalein clearance, bilirubin tolerance), and metabolic function (glucose tolerance, galactose clearance), The most common tests used in toxicology are the serum alkaline phosphatase and serum transaminases, and in some cases a dye clearance (bromsulphalein) or glucose tolerance.

The kidneys are responsible for excretion of certain substances, e.g., urea, and for concentration and dilution of urine. Tests for excretion involve dyes like phenolsulphonphthalein and also measure such substances as urea and creatinine, Concentration and dilution tests involve measurement of urine specific gravity after fasting for various periods. These tests are generally not used in toxicology screening studies unless there is reason to believe the toxicant acts on the kidneys.

The evaluation of these tests may proceed with or without modification of the tissue metabolism. That is, promotors and inhibitors of enzyme systems, e.g., SKF-525A for mixed function oxidase, may be used to enhance the susceptibility of a particular organ or system to damage from a toxicant. This in effect maximizes the response so that the toxic action can be more readily observed.

Many other specific tests are available for evaluating various organs and systems such as sperm motility, specific gravity of cerebrospinal fluid. calcium-phosphorus ratios for the skeletal system along with tensile strength and compaction, epinephrine sensitivity of heart muscle, acetylcholine test of lungs, metabolism of excised tissue, work and strain measurements of the various muscle systems, etc.

\section{Behavioral Effects}

A recent addition to the field of toxicology has been behavioral testing. Testing methods have been devised for everything from simple perception to complex tasks involving perception, learning, judgment, motivation, and motor activity. The value of the behavioral methods lies in the ability of the nervous system to respond to toxic agents a $\mathrm{t}$ doses much lower than those necessary to produce "classic" signs of toxicity in the organism. Therefore, these methods are a potential sensitive indicator of hazard and can be used as an "early warning system. "Several good reviews of behavioral toxicology are available (34-37).

Some of these methods rely on newer methods of analysis such as contingent negative variation (CNV). Some are really an application of preexisting principles such as dorsal evoked potentials and neuromuscular transmission time that have been widely used by experimenters in the field of neurophysiology. Most of these techniques have only recently been turned to the evaluation of toxicity.

At the present time, standardization and validation of behavioral techniques has not been accomplished. The question often raised by regulatory agencies is how do you relate an observed behavioral decrement to an adverse health effect, especially if there is no concurrent structural change apparent in the nervous system. Because of these factors, behavioral studies are often derogated by these agencies when setting exposure limits for toxicants. Current research is being conducted, however, under Government contracts to answer some of these questions.

\section{Comparison of Short- and Long-Term Methods for Systemic Toxicity}

Most of the procedures noted in this section are equally applicable to short- and long-term testing, obvious exceptions being irritation and sensitization tests. The value of long-term testing for systemic toxicity lies in the ability to use low doses that do not produce detectable adverse effects in a short time period to see whether bioaccumulation and cumulative effects occur. Predictions of the effect of bioaccumulation can be made knowing the effects of short-term high doses, but final evaluation of the toxicity depends on the longterm effects observed, As will be pointed out in the metabolism section, toxicants can be potentiated or inhibited by the metabolism and relative accumulation of the toxic moiety. Without definitely knowing the various metabolic reactions, rates, and probabilities, it is impossible to accurately predict toxic effects. High short-term doses may induce a toxic reaction, such as death from pulmonary edema, that might mask longterm, low-level exposure effects such as liver cancer, The differences in short-term and longterm tests for systemic toxicity include the number of interim measurements allowed, the ability to ascertain the types of effects which might develop only over a long period and the progression or time course of toxic manifestations, and 
the ability to evaluate mechanisms of bioaccumulation or adaptation in the organism.

\section{Mutagenicity}

Rapid identification of a food contaminant as a possible mutagen is necessary to reduce the potential genetic risk to humans who might contact the contaminant. Mutagenic effects on humans often cannot be directly detected, and deleterious effects on the human gene pool may not become apparent for many generations if, for instance, the deleterious effect is due to a recessive gene. Heritable genetic damage in humans may result from any of several types of effects on the genetic material, The two major classes of effects are point mutations, which generally affect a single gene or part of a gene, and more extensive chromosomal effects such as gross changes in structure or changes in number.

Only a few tests are available that directly evaluate genetic effects of exposure of mammals to chemicals: however, the potential of a chemical to produce heritable genetic alterations in man can be evaluated indirectly from its effects on genetic material in various biologic test systems, including micro-organisms, mammalian cell cultures, insects, and intact mammals.

For substances that cannot feasibly be eliminated from the human environment, it is not sufficient to identify the existence of a genetic hazard; quantitative assessment of the risk involved is necessary for appropriate regulatory activity, such as establishing action levels or tolerances for food contaminants.

Mutagenicity testing is also used to prescreen chemicals as an indicator of carcinogenic potential and, less frequently, other toxic effects such as teratogenicity. This application is based on empirical demonstration or correlation between mutagenicity and carcinogenicity of chemicals (38) and does not depend on the assumption that the same mechanism is involved in both types of effect.

\section{Approaches to Testing}

As in all toxicological tests, mutagenicity tests may produce false negatives (a negative result when the substance is actually mutagenic) and false positives (a positive result when it is not mutagenic), and correlation between the results from two test systems may be poor. Ideally, a mutagenicity test system should be sensitive enough to detect any chemical that may cause heritable genetic damage and its results should be repro- ducible. Finally, the test results should be quantitatively applicable to mutagenesis in humans. Since no single test can fulfill these requirements and none is reliable enough to stand alone as an indicator of mutagenic potential, mutagenicity 'testing should include a variety of systems selected to show whether the test substance or its metabolize produce any of a range of genetic effects. The test battery approach includes systems that will detect several types of gene mutations, chromosomal aberrations, and DNA repair; thus, this approach offers the greatest reliability for determining mutagenic potential, Tests that evaluate effects in intact mammals are essential for predicting mutagenicity in humans.

Since screening large numbers of chemicals by the test battery approach $(39,40)$ may be prohibitively costly, a hierarchical approach to mutagenicity testing, known as tier testing (41), has been suggested. Tier 1 consists of relatively inexpensive short-term prescreening tests. These use micro-organisms or other in vitro systems to determine priorities for indepth testing. Substances that produce positive results in these tests, as well as those that are negative but are structurally similar to known mutagens or to which there is a substantial risk of exposure for humans during or preceding their reproductive years, should continue into Tier 2 .

Tier 2 tests are usually designed to detect substances that are not mutagenic in vitro but are metabolized to an active form in the intact mammal. Tests used at this level may include the dominant lethal test, in vivo cytogenetic tests, the hostmediated assay, and body-fluid analysis. Substances that are negative in Tiers 1 and 2 are generally considered safe for use and are given very low priority for further testing.

Only substances for which it is important to assess risk are subjected to Tier 3 testing, designed to permit quantitative evaluation of mutagenic potential. Tests used at this level include multigeneration mammalian studies, such as the heritable translocation test, $\mathrm{X}$ chromosome loss test, and specific loci test in mice.

Tier testing may represent an efficient use of resources in large-scale mutagenicity testing, but the use of prescreening tests carries serious disadvantages in determining mutagenic potential, If test chemicals are prescreened by a single microbial test, the proportion of false negatives may be unacceptably high, and potentially hazardous or useful substances may escape further testing. The use of two or three tests at this level, including both micro-organisms and mammalian 
cell cultures with and without activation by mammalian enzyme systems, may substantially increase the reliability of prescreening (96). Nevertheless, such cell systems may not approximate metabolic events in the intact mammal closely enough to reveal the mutagenic action of some substances that are potential human mutagens.

Whatever the testing approach and test systems selected, mutagenicity tests should include a positive control as well as negative (untreated and solvent) controls. The positive control substance, a known mutagen in animal systems that is selected for its structural similarity to the test chemical, serves to demonstrate the sensitivity of the test organism and the efficacy of the metabolic activation system used.

\section{Current Test Systems}

Chromosomal effects of many substances have been demonstrated in plants such as Vicia faba and Tradescantia (42), and the latter organism has also been used in detection of somatic mutation (43). While the genetic events involved (alterations in DNA) are the same as those in mammalian cells, their relevance to human mutagenesis has been questioned because of the major phylogenetic and physiologic differences between plants and animals; thus, a negative result in plants does not indicate that a substance is not a mutagen in mammalian systems.

Of the many bacterial species that have been used to detect point mutations, the most extensively employed are the Salmonella typhimurium mutants developed by Ames $(44,45)$. The Ames test uses a series of histidine-requiring mutant strains that revert to histidine-independence by specific mechanisms, either base-pair substitutions or frameshift mutations, The original strains have undergone several further modifications that increase their sensitivity to mutagens by interfering with DNA repair or modifying the cell wall to enhance the penetration of chemicals into the cell. Bacteria treated with the test chemical are plated on selective media or cultured in liquid suspension to determine the number of revertants. A reproducible mutation rate twice the spontaneous (control) rate is usually considered evidence of mutagenic activity.

Because microbial cell systems do not possess the metabolic capabilities of mammals, they will not detect chemicals that exert a mutagenic effect through metabolic intermediates, Several activating systems have been developed for use with in vitro test systems to duplicate the effects of mammalian metabolism. The most extensively used means of metabolic activation is the addition of microsomal mixed-function oxidase enzymes, typically from rodent liver homogenates, to metabolize the test chemical in vitro, This activating system is added to the culture medium as part of the Ames testing procedure with S. typhimurium, and it has provided evidence for the mutagenicity of many substances that have no direct mutagenic effect on these bacteria (51). Microsomal enzyme activation is also used with other microbial test systems (46-48). The major drawbacks of this system are that it would not detect chemicals metabolized to mutagenic intermediates by mechanisms other than liver microsomal enzymes, e.g., substances metabolized by the intestinal flora, and it is possible that the in vitro metabolism of the substance does not adequately mimic its metabolism in the intact organism because of competing reactions. Another drawback is that a standardized in vitro activation system has not been devised to date.

Another widely used bacterial system is the multipurpose strain of Escherichia coli developed by Mohn and coworkers (49). This strain can be used to measure reverse mutations restoring the ability of the bacteria to synthesize the nutrients arginine and niacin. Forward mutation rates in two genes controlling galactose metabolism can also be scored in this strain of E. coli. Use of this test organism permits the detection of several types of mutation in a single experiment.

Eukaryotic micro-organisms that are used to detect the ability of chemicals to produce point mutations include haploid strains of the yeasts Saccharomyces cerevisiae and Schizosaccharomyces pombe $(47,50)$ and of the ascomycete $\mathrm{Neu}$ rospora crassa (51). A diploid strain of S. cerevisiae permits detection of chromosomal damage expressed as mitotic recombination that produces phenotypic color changes (52).

Whole-animal activation mechanisms can circumvent this problem but they are generally much less sensitive than tests using in vitro activation. In these systems, rodents are exposed to the test chemical by an appropriate route, and the effect of rodent metabolizes on microbial genetic markers is determined by either body-fluid analysis or host-mediated assay procedures,

In the body-fluid analysis (53-56), the microorganisms are treated with the urine, blood, or homogenized tissues of the exposed animals. Caution is necessary in interpreting negative results of these studies, in the absence of supplementary pharmacologic data, since even if the chemical is metabolized to a mutagen, other factors such as 
tissue-specific activation and detoxification mechanisms and the half-life of the compound and its metabolizes may affect test results. In the hostmediated assay $(57,58)$, the micro-organisms are exposed to mammalian metabolic products of the test substance by being introduced into the peritoneal cavity, circulatory system, or testes of the host mammal. The host is treated with the test substance, and after an appropriate incubation period, the indicator organism is removed and examined for mutations.

Genetic damage in micro-organisms can also be assessed indirectly through the use of DNA repair-deficient strains of bacteria $(44,59)$. These tests organisms and otherwise identical strains that have normal ability to repair DNA are treated with the test substance. Toxic action of the test substance produces zones in which bacterial growth is inhibited, and the difference in size between the inhibition zones in repair-deficient and normal strains indicates the extent to which this toxicity is due to damage to the DNA.

A number of mammalian cell systems in culture have been developed for detecting point mutations, including cell lines derived from mouse lymphomas, Chinese hamster ovaries and embryos, and human fibroblasts and lymphoblasts (60-63). In addition, gross chromosomal changes such as breaks, gaps, and rearrangements can be microscopically observed in these cells. Stable rearrangements, such as translocations and inversions, are considered evidence of heritable changes. The induction of only gaps and breaks is not regarded as evidence of mutagenicity, because these aberrations often occur as a result of general cytotoxicity and thus may be present only in moribund cells. Like microbial systems, in vitro mammalian cell tests can be used in conjunction with activation by mammalian enzymes or with whole-animal activation to permit detection of mutagenic effects by metabolic products of a test substance.

Mammalian cells, including human white blood cells, are also used to detect chemical damage to DNA by measuring unscheduled DNA synthesis (63). This indirect indicator of genetic damage is evaluated by measuring the uptake of radioactive thymidine for repair of damaged DNA during those stages of cell growth when DNA synthesis does not normally occur. A similar test with mouse spermatocytes exposed in vitro or in vivo demonstrates effects on DNA in germinal cells (64),

Sister chromatid exchange, a reciprocal exchange of segments at homologous loci. measured by autoradiographic methods, has also been used to examine a variety of chemicals $(65,66)$. Sister chromatid exchange in various cell systems has been demonstrated following exposure to known mutagens, but additional work is needed to define the extent to which results are correlated with more traditional mutagenicity tests,

The fruit fly Drosophila melanogaster is used in a comprehensive and extensively characterized mutagenicity test system which can detect all types of mutagenic activity at a fraction of the time and cost of in vivo mammalian testing (67). The large number of genetic markers and known chromosomal aberrations make it possible to assay a chemical for many types of mutagenic activity in a single test. The sex-linked recessive lethal test (68) in Drosophila is a very efficient mutagenicity assay, since about 20 percent of the insect's genetic material is located in the $X$ chromosome. Recessive lethal changes caused by point or chromosomal mutation can be mapped and in most cases the nature of the change causing the mutation can be determined. This test also permits a quantitative assessment of mutagenic activity. Because of the large size of Drosophila chromosomes, this organism can also be used readily to assess meiotic and mitotic recombination, dominant lethality, translocations, and deletions. Some indirect mutagens that required metabolic activation have been shown to be mutagenic in Drosophila, indicating that these insects have a microsomal mixed-function oxidase system (69). However, to determine whether Drosophila test results are useful for risk assessment, more information is needed on how their metabolism of foreign chemicals compares to that in humans. A few other species of insects have also proven useful in mutagenicity testing, including 'several species of the parasitic wasp, Habrobracon (70).

Clearly the most accurate predictions of mutagenic potential in humans can be drawn from tests that determine direct genotypic and phenotypic effects in mammals exposed to the test chemical by routes relevant to human exposures, Several direct mammalian tests exist, but these have the disadvantage of detecting only a few of the possible types of genetic damage. Chromosomal damage occurring in vivo can be detected in several different cell types, such as bone marrow cells and circulating lymphocytes, and the presence of micronuclei in red blood cells (7 I). Cytogenetic tests using mammalian lymphocytes and the micronucleus test offer the advantage of permitting direct comparison with effects on humans resulting from accidental exposures; how- 
ever, these tests demonstrate only effects on somatic cells and do not provide direct evidence of heritability.

Cytogenetic changes in mammals can also be evaluated in germinal tissue from the testes. In the direct spermatocyte test (72), male mice are exposed to the test substance. After sufficient time for the treated spermatogonia to reach the spermatocyte stage, they are examined for cytogenetic abnormalities. This test allows for the actual observation of induced cytologic changes in premeiotic male germ cells, but it does not permit detection of effects in postmeiotic cells or their transmission to the offspring.

Effects on offspring can be evaluated in mice by the heritable translocation test (73) and the $X$ chromosome loss test (74). In the heritable translocation test, $F_{1}$ male offspring of treated mice are mated to determine sterility, and indication $o$ possible translocation heterozygosity. Chromosomal effects are then confirmed by cytogenetic analysis of the germinal cells of the male of offspring. The $X$ chromosome loss test permits the detection of chromosome loss resulting from nondisjunction in the female, since, unlike somatic chromosome aneuploids, animals of $\mathrm{XO}$ genotype are usually viable, Aneuploidy for the $X$ chromosome can be detected by genetic markers and confirmed by cytologic observations.

The dominant lethal assay, usually performed in the rat or mouse, uses fetal loss as an indicator of induced chromosomal mutations in male germinal cells (75). The death of the zygote is assumed to result from chromosomal abnormalities in the sperm of male mice exposed to the test chemical. This test is relatively easy to perform and its results have been positively correlated with mutagenicity in other animal systems. Preimplantation loss alone is not used as an indication of mutagenicity since it has been found to occur for reasons other than chromosomal changes in the sperm. Disadvantages of this test are its relative insensitivity and difficulty in clearly distinguishing weakly positive results.

Only one test is available at present that can detect heritable gene mutations induced in mammalian germ cells. In the specific locus assay in mice, forward mutations at seven loci, affecting characteristics such as coat and eye color are mated with mice homozygous for recessive alleles at these loci (75). Because such a small number of loci are involved, this test required the scoring of 20,000 to 30,000 offspring at each dose level to produce reliable results and is therefore very costly and time consuming.

\section{Carcinogenicity}

In the event of massive or long-term environmental contamination of food destined for human consumption, one of the decisions to be made is whether the contaminant appears to pose a significant carcinogenic risk. With this in mind FDA submits the candidate compound to the Chemical Selection Working Group at the National Cancer Institute for consideration under the carcinogen bioassay screening program $(76,77)$.

\section{Prerequisites for a Carcinogenicity Study}

Once the compound has been selected, it is screened using a chronic or lifetime exposure regimen $(76,78,79)$. However, before the long-term study is undertaken, specific toxicologic profiles must be obtained. Young healthy adult animals of each sex and strain to be used in the long-term studies should be used in the preliminary studies. The animals should be of uniform age and weight and should be tested using the same formulation and route of exposure to be used in the long-term studies. The first is an acute study designed to gain additional information on the acute toxicity, assuming there is a paucity of data on this aspect of toxicity, and to determine the lethality of the test compound. The duration of this test should not exceed 24 hours and should include at least three dose levels determined by a geometric progression. one of the dose levels selected should represent the highest dose to be used in subsequent studies. Throughout the investigation, all relevant clinical signs should be recorded. Necropsies should be performed on a random selection of animals of each sex and strain, and any abnormal histopathologic changes should be noted.

After the 24-hour study, a 14-day investigation should be initiated in an effort to ascertain the doses necessary for the subchronic study, the next prerequisite investigation for the chronic study. This toxicologic study requires five dose levels, with the highest one, estimated from the 24-hour acute study, producing no more than 10percent lethality. The other dose levels should represent geometric decrements of the highest dose. Animals should be treated with the test substance for no more than 14 days, held another 24 hours, and then sacrificed for necropsy. Throughout the study, the animals should be observed for clinical signs of toxicity. Other toxicity data, such as those derived from organ function tests and metabolism studies, are also necessary. 
The next toxicity study involves the administration of the test substance for 90 days and is used as a predictor of the maximum tolerated dose (MTD). This can be defined as the highest dose given during a chronic study that can be predicted to not alter the animals' normal longevity from effects other than carcinogenicity, In practice, MTD is considered to be the highest dose that causes no more than a 10-percent decrement in weight compared to controls. Five dose levels are required in this study, with a minimum of 10 animals of each sex and strain in each dose group. The highest dose level used should be the lowest concentration that produced any detectable untoward toxic effects in the 14-day study. The remaining dose levels should be determined as in the 14-day study, If the selected dose levels do not produce a discernible no-effect level, the study should be repeated with lower doses.

\section{Carcinogenic Bioassay}

The chronic study $(76,78,79)$ represents the essence of the carcinogenicity bioassay. It is used to determine the carcinogenicity of a compound in males and females of two mammalian species, usually the rat and mouse. The species selected are tested throughout their entire lifespan. Each test group should consist of a statistically representative number of animals. The highest selected dose should represent MTD and the remaining dose levels should be adjusted accordingly. There should be at least one control group, in which the animals receive only the vehicle used for administration of the test material, If no vehicle is used, this control group should be untreated but identical in every way to the experimental groups. In addition to the concurrent control group, a colony or historical group should be used for the comparison of longevity, spontaneous diseases, and spontaneous tumor incidence. The historical control may also be used for statistical comparisons. In some studies, a positive control group that has been treated with a compound structurally similar to the test compound and known to be carcinogenic in the test species may be indicated. However, because of the added risk of handling a known carcinogen, a positive control group is seldom used.

Throughout the study, animals must be observed for signs of toxicity. Every animal should be examined carefully each week, Animals should be weighed and food consumption measured. In some cases, it is desirable to evaluate tissue distribution and concentration of the substance or its metabolizes.
The animals in any one test group should be sacrificed at an adjusted or prearranged date. However, a group can be terminated earlier if there has been high cumulative mortality. Moribund animals should be sacrificed immediately upon discovery to lessen the likelihood of unobserved deaths and subsequent autolysis or cannibalism. Control groups should be sacrificed according to the original or adjusted sacrifice date; the later date is preferred. Because of the strong dependency on histopathologic results and to avoid possible criticisms of the study, necropsies and histopathologic examinations should follow standard procedures required by regulatory guidelines.

The major drawback to the carcinogenic bioassay procedure is the time and cost required to complete and analyze such a study. If the study is done properly, however, the results should be conclusive and for the most part indisputable, although there still remains the question of extrapolation of results to the human population.

\section{Short-Term Testing as a Prediction of Carcinogenicity}

Evaluation of carcinogenicity has generally relied on the results of long-term animal studies, To use this kind of testing approach for every substance suspected of being carcinogenic would be cost-prohibitive and certainly impractical in terms of the overall time required to test all suspected chemicals. Therefore, short-term tests are being developed to identify carcinogenic substances. There has been much criticism concerning the comparison of short-term testing results from different laboratories because of the varying conditions and refinements in techniques practiced among testing facilities. The protocols for these short-term tests, especially those involving mammalian enzyme activation systems, have not been standardized or validated through interlaboratory comparative testing procedures; thus, comparisons of the data from one laboratory to the next have often produced conflicting conclusions. In this light, a study conducted in one laboratory that compares several short-term testing systems has added importance in clarifying the relative usefulness of the compared systems,

In a recent study by Dr. Ian Purchase (80), 120 organic chemicals (50 known carcinogens and 62 noncarcinogens, based on published experimental data) were evaluated for activity in six shortterm test systems. These systems included: 1) mutation of Salmonella typhimurium (45), 2) cell transformation (81), 3) degranulation of endoplas- 
mic reticulum (82), 4) sebaceous gland suppression (83), 5) tetrazolium reduction (84), and 6) lesion formation after subcutaneous implant (85). Four additional tests used in a preliminary study were found to be insufficiently accurate or sensitive to justify a full evaluation. The tests rejected were transplacental blastomagenesis (86), piperidine alkylation (87], iodine test (88), and the acridine test (89).

Although there were considerable variations between tests in their ability to predict carcinogenicity, two tests were quite accurate in distinguishing between the known carcinogens and the noncarcinogens. These were the cell transformation test and the bacterial mutation test, which had accuracies of 94 and 93 percent, respectively. The use of cell transformation and bacterial mutation together provided an advantage over the use of either alone, predicting 99.19 percent of carcinogens. Not surprisingly, the inclusion of the other four tests in a screening battery with these two resulted in an improved ability to detect carcinogens (99.97 percent), but greatly decreased the accuracy and discriminatory value of the battery. It is important to note that all tests generated both false positives and false negatives: the percentages for both can be readily calculated by subtracting the positive predictability values from 100 percent.

A description of each of the tests, with comparative percent accuracies for predicting carcinogenicity as determined by Purchase et al. (80) is as follows:

- Bacterial mutation. The procedures used were those of Ames, in which four strains of S. typhimurium (TA 1535, TA 1538, TA 98, TA 100) were tested with each compound in an assay medium containing a metabolic activation system composed of rat liver postmitochondrial supernatant (S-9 fraction) and cofactors. The overall accuracy of the test in predicting the carcinogenicity of the compounds in this study (80) was 91 percent for carcinogens and 94 percent for noncarcinogens. These figures agree with the previously published value of 90 percent by McCann et al. (90) but are considerably higher than those published by Heddle and Bruce (91) who found 65 and 81 percent, respectively.

- Cell transformation. The procedures used were those of Styles (81) and involved three types of mammalian cells-human diploid lung fibroblasts (WI-38), human liverderived cells (Chang), and baby Syrian hamster kidney cells (BHK 21/cl 13). In all assays, the cells were used with and without metabolic activation with S-9 fraction as described previously. Without activation, very few carcinogens transformed hamster or human cells in the period of study. With activation, all cell lines detected carcinogens with an accuracy of 88 percent or better. Furthermore, by the use of both the hamster cells and either of the human cells, the overall accuracy was improved to 94 percent (91 percent for carcinogens and 97 percent for noncarcinogens).

- Degranulation. The procedure used is that of Williams and Rabin (82) and the test is commonly called the Rabin test. The test measures the loss of ribosomes (degranulation] from isolated rat liver endoplasmic reticulum following incubation with the test compound. The overall predictive value was 71 percent for both carcinogens and noncarcinogens.

- Sebaceous gland test. The procedures used were those of Bock and Mund (83) where test chemicals were applied directly to the skin of mice and a depression in the ratio of sebaceous gland to hair follicles indicates a positive response. The overall predictive value of the test was 65 percent $(67$ percent for carcinogens and 64 percent for noncarcinogens).

- Tetrazolium reduction. The procedures used were based on those described by Iversen and Evensen (84). Test solutions were applied directly to the skin of mice and the skin samples were incubated in tetrazolium red solution. An increase in the in situ biologic reduction of the colorless tetrazolium to a colored formazan compound indicates a positive response. The overall predictive value of this test was 57 percent (40 percent for carcinogens and 71 percent for noncarcinogens).

- Subcutaneous implant. The procedures used were essentially those of Longstaff and Westwood (85] and involved the subcutaneous implantation of a filter disc overlaid with a gelatinous suspension of the test $\mathrm{c}_{\mathrm{om}}{ }^{-}$ pounds into mice. After 3 months, the surrounding tissues were scored for lesions. The overall predictive value for this test was 68 percent (37 percent for carcinogens and 95 percent for noncarcinogens).

While the work of Purchase and associates (80) does present a very salient assessment of the more pertinent in vitro assays, it also points out 
the shortcomings of this type of approach. Shortterm tests do not use the induction of cancer as an endpoint, but each has a parameter, such as induction of a point mutation, that varies with the carcinogenicity or noncarcinogenicity of the test substance. Accordingly, the authors feel that these test parameters should not be given a greater weight than that of any other arbitrary response, regardless of how biologically significant any of these tests might appear to be with respect to the theories of the chemical induction of cancer. Also, however much generalized data might be generated to support the predictive accuracy of the given test, this accuracy should not be assumed to apply uniformly to compounds of every chemical class,

\section{Teratology and Effects on Reproduction}

An investigation of a teratogenic agent involves the study of congenital malformations other than those that are inherited. Teratogens themselves act as triggers for malformation induction. Malformations may include gross, histological, molecular, and behavioral anomalies,

The sensitivity of an animal to a teratogen is determined by: 1 ) the period in which the insult is received during the gestation period (this includes before germ layer formation and during embryogenesis or organogenesis); 2) the dose and the route of administration of the compound; 3) placental transfer of the suspect teratogen, including its lipid volubility, protein binding ability, and metabolism and 4) uterine and dietary factors.

A toxicologic profile of a suspect teratogen requires an evaluation of potential hazards to reproduction and, particularly, to developmental processes that respond to environmental insult through mutation, chromosomal aberrations, mitotic interference, altered nucleic acid synthesis, enzyme inhibition, and altered membrane characteristics. This is particularly important in the conceptus, embryo, and the neonate where the biochemical, morphologic, and physiologic properties change rapidly. Thus risk assessment must not only address the teratogenicity of a contaminant but also its effect on reproduction.

\section{Classical Approach}

For an adequate teratologic assessment (79,92, 93), exposure to the toxicant should parallel as closely as possible that expected in the human population. The pharmacologic activity of the compound as well as its acute and chronic toxici- ty is also a consideration. For teratogenic studies, the toxicant is usually administered daily on the specific days of gestation representing the period of greatest sensitivity. Administration of the test substance should begin at or before implantation and should continue throughout the period of major organogenesis.

The selection of an animal species for evaluation of teratogenicity is an important consideration. Test protocols currently in use recommend at least two mammalian species, the first being a rodent (e. g., mouse. rat, hamster) and a nonrodent mammalian species (e.g., rabbit). One species should be the same as that used in the test for reproductive effects.

In conducting the teratogenic investigation, at least three dose levels should be used, along with concurrent control groups. These control groups should consist of untreated animals, animals treated with the vehicle of administration only, and, possibly, animals treated with an agent that is known to cause the effect that is being investigated. The use of historical or colony controls may also be helpful in evaluating the data. Both test and control animals must be young, mature, prima gravida females of uniform age, size, and parity. The control groups should be handled and maintained like the test groups. The highest dose level to be considered should produce signs of embryo or fetotoxicity as suggested by fetal growth retardation and more significant-

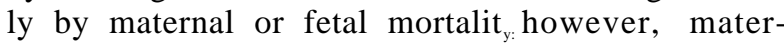
nal mortality should not exceed about 10 percent. The other dose levels can be obtained in a decreasing logarithmic fashion to a suspected no-observable-adverse-effect level. In the classical teratology study, treatment may be by gavage so that the administered dose is accurately known. It has been shown, however, that use of this route of administration may induce anomalies in progeny that are a consequence not of the test compound but of the stress of dose administration (94).

Prior to initiating the study, it is important to determine whether sires and dams have successfully mated, This includes examinations for the presence of plugs or evidence of sperm in vaginal smears. Throughout the study, females should be observed for behavioral changes, food and water consumption, body weight, vaginal bleeding indicating possible abortion, and spontaneous deaths. Females showing signs of aborting or de-

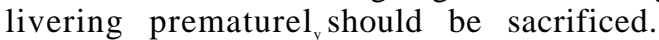

Fetuses should be obtained 24 hours before anticipated parturition by cesarian section, Dams should be sacrificed and a complete necropsy per- 
formed with emphasis on the reproductive organs. Data to be obtained include maternal weight and weight of the gravid uterus, the number of fetuses, location of fetuses within the uterine horn, number of corpora lutea, the number of sites of resorptions (either early or late), the number and weights of live and dead or moribund fetuses, the number of dams having dead fetuses or showing reproductive wastage, and any internal or external anomalies through appropriate procedures (complete necropsy or Alizarin Red staining). Approximately one-half to two-thirds of each litter should be screened for skeletal anomalies while the remainder of the litter should be screened for aberrant soft-tissue effects. The significance of each observed anomaly should be evaluated relative to its natural incidence and its occurrence in controls.

\section{Effects on Reproduction}

The intent of the reproductive study is to determine whether the substance under consideration produces demonstrable effects from mating through lactation, in pregnancy, or in growth and development of progeny from conception through maturity $(79,92)$.

Reproductive studies are normally performed in at least two mammalian species, one of which should be the same as one of the two species used for teratogenic evaluation. For rodents, at least three dose levels are selected in addition to the controls. The highest dose must produce an observable effect but no more than 10-percent lethality, whereas the lowest dose should yield no demonstrable adverse effects. The control groups used in reproduction studies are similar to those discussed for other studies, namely a vehicle control and possibly a historical control.

The period of dosing and observation for these studies is rather long. i.e., about 1.5 years. The test material is administered to the $F_{0}$ and the $F_{2}$ generations. The third generation of animals $\left(\mathrm{F}_{2}\right)$ is then exposed in utero and through weaning.

Dosing of the $\mathrm{F}_{\text {" generation should begin as soon }}$ as possible after weaning and definitely before the animals (males and females) are approximately 6 weeks of age. The substance under test should be administered daily to the $F_{1}$ generation for approximately 100 days before breeding; dosing should continue in the females that have been bred and have delivered progeny until all of the $\mathrm{F}$ generation have been weaned.

At approximately 30 days of age, a statistically valid number of animals, males and females, from the $F_{1}$ generation are selected and exposed to the test material for at least 120 days. At this point they are bred to produce the $F_{2}$ generation.

The types of data to be collected include growth and time of delivery for each weanling as well as overt signs of toxicity, the general behavior and condition of the mothers, measurements of spermatogenesis in all $F_{1}$ generation males used to produce the $F_{2}$ generation, litter size, number of stillborn/live births, and any physical or behavioral anomalies.

A statistically valid number of animals (males and females) obtained from the $F_{1}$ generation and used to produce the $F_{2}$ generation should be sacrificed and examined at the appropriate time, with special emphasis on the histopathologic state of the reproductive system. In addition, an adequate number of weanlings of each sex from each dose level, including controls, should also be sacrificed and used for histopathologic analyses.

Data derived from the above should be evaluated for the existence of a relationship between exposure and the incidence and severity of effects on reproduction and behavior, tumors, and mortality. The no-observable-adverse-effect level should also be determined.

Compared to a teratology investigation, which requires about 3 months to complete the exposure and to analyze the results, a three-generation reproduction study represents a considerably longer expenditure of time, approaching 1.5 years between the initiation of the study and the completion of the analysis of the gathered data. However, an elementary profile on teratogenicity and reproductive performance can be obtained within a period of a year or less as a part of a continuing long-term toxicity study by adding the appropriate number of animals at the beginning of the long-term study (2).

\section{Metabolism}

Metabolic assessment studies are not directly used for the assignment of risks, tolerances, or action levels, These studies are used to determine parameters, such as absorption, distribution, storage, and excretion, that may affect the performance of materials in biologic systems, thus enabling the researcher to design testing protocols that measure the overall effect of exposure to the material rather than just measuring a part of the biologic response. These protocols can then take into account such factors as tissue concentration, length of time in contact with specific organs or tissues, ease of reactivity, and the production of significant (or nonsignificant) changes 
in overall body concentrations that may alter the outcome of a specific testing regimen.

Metabolic study systems are usually centered around the concept that the circulatory system is the major means of transportation of the material, regardless of the route of exposure, Although specific materials may be readily metabolized at or near the site of entry into the body (e.g., the lungs, skin, and intestines), the majority of materials are transported unchanged to the liver and then distributed via the circulatory system to other tissues and organs. At the same time, some of the material may be excreted unchanged in the urine, feces, and air or converted to various metabolizes that are excreted or bound in the tissues. The proportions of the metabolic products that are excreted or bound depends on the chemical nature of the original compound, the dose, route of administration, species, strain, sex, diet, and environmental factors.

The objective of the metabolic study is to mathematically evaluate the rates and relative importance of these processes in limiting the concentration of materials in the tissues of the body. For this purpose, the body is usually visualized as a group of pharmacokinetic compartments, a simplistic view that surprisingly approximates fairly accurately the complex, interdependent processes that actually occur in the body (95). These compartmental models allow the researcher to use measurements of blood concentration as indirect estimates of tissue concentrations and to determine the length of time the material remains unaltered (i. e., the biologic half-life). Rates of absorption from one compartment to another can also be readily measured, thus giving the researcher information on the rate constants of diffusion into tissues and on the rates of distribution from the blood to various tissues. Clearance or elimination rates can also be determined for the excretion of the material from the body via the kidneys, gastrointestinal tract, lungs, saliva, and perspiration.

The rate of metabolism of materials depends on many factors, among the most important of which are the physiochemical characteristics of the molecule itself. Polar compounds are usually excreted very rapidly from biologic systems largely unchanged because of the chemical activity of these compounds, whereas nonpolar compounds such as lipids usually remain in the body longer because they must be metabolized to polar compounds before excretion occurs.

Another important factor in the metabolism of a compound is its structural resemblance to natu- rally occurring substances in the body. Foreign compounds that closely resemble normal body constituents are frequently metabolized by the same specific enzyme systems that metabolize their normally occurring analogues. Most foreign compounds, however, have no endogenous counterpart and must be metabolized by relatively nonspecific enzyme systems. These nonspecific enzymes catalyze many different types of reactions leading to a diversity of metabolic products. In general, the nonspecific enzyme reactions can be categorized into two types, The first includes the conversion of one functional group into another (oxidation of alcohol to aldehyde), the splitting of neutral compounds to fragments having polar groups (hydrolysis of esters and amides), or the introduction of polar groups into nonpolar compounds (hydroxylation). The second type includes the conjugation of the created polar group with glucuronate, sulfate, glutathione, or methyl groups to form a soluble, excretable product. The product formed in the first reaction may be either more or less toxic than the parent compound or may possess a different type of toxicity. Often, it is this product that actually causes the toxic effects, including cancer, mutations, cellular necrosis, hypersensitivity, fetotoxicity, and blood dyscrasias. A portion of the chemically reactive metabolize formed becomes bound to tissue macromolecules, such as cellular proteins, DNA, RNA, glycogens, or lipids. In this way, it disrupts the normal function of the macromolecule, causing adverse biologic effects. In contrast, the majority of the second type of reaction products are usually either nontoxic or considerably less toxic than the parent compound,

The metabolism of a foreign material is controlled by enzymes, and any factor which affects these enzymes also affects the metabolism of the compound and consequently its toxicity. The metabolism of a compound may be inhibited or stimulated by the presence of competing substrates. Pretreatment with drugs, steroids, food additives, pesticides, polycyclic hydrocarbons, polycyclic amines, and normal constituents of food can result in an increase in the activity of enzymes that metabolize foreign compounds. This increase in enzyme activity differs according to the inducing chemical, but is mediated through an increased rate of synthesis of enzyme protein, a decreased rate of turnover of enzyme, or an activation of enzyme, possibly by changes of structure or conformation. Because of this phenomenon, chronic administration of a foreign material may enhance 
the activity of the enzymes that catalyze its metabolism.

Several types of biologic phenomena are readily studied using a metabolic test system. These include activation, antagonism, synergism, and potentiation. Activation has been briefly touched on above with the description of the process of toxification: it involves the rendering of an inactive molecule into an active molecule, usually through the removal or substitution of a neutralizing factor attached to the molecule, but it may also involve the direct addition of a constituent to the molecule. One of the most common examples of activation, the conversion of a precarcinogen to a carcinogen, is the addition of oxygen molecules to polycyclic aromatic hydrocarbons via the microsomal NADPH-dependent cytochrome P-450 mixed function oxidase enzyme system. This conversion to the oxide produces a carcinogenic agent of considerable potency, whereas the parent material does not possess a direct carcino- genie potential per se. The determination of metabolic activation can permit the experienced researcher to predict the kind of adverse effect that is likely to be elicited from the parent molecule, thus enabling the researcher to better design experiments to observe these effects.

From a properly designed and well-carried-out metabolic study, the researcher can gain valuable insight into the potential toxic actions and biologic effects to be expected from a foreign compound. If a compound is not absorbed or if the compound is destroyed or rapidly eliminated from the body there is little likelihood of pronounced toxic effects. If the principle metabolic products are polar, conjugation (and thus elimination) rapidly occurs and again there is little potential for pronounced toxicity. On the other hand, if metabolism produces an activation product, toxicity is enhanced and biologic effects may be pronounced.

\section{APPENDIX c REFERENCES}

1. 41 CFR 51206 (1976).

2. Food and Drug Administration Advisory Committee on Protocols for Safety Evaluations: Panel on Reproduction Report on Reproduction Studies in the Safety Evaluation of Food Addit ives and Pest iicicle Residues ( 1970). Tox.Appl.Pharm. 16:264.

3. Modern Trends in Toxicology (1968). E. Boyland and R. Goulding, eds., But terworths, London.

4. Methods in Toxicology (1970). G. E. Pagett, cd., Davis, Philadelphia.

5. Frawlev, J. P. (1955). Food Drug Cosmetic Law J. 10: 703 .

6. Smyth,H.F.,Jr., and C. P. Carpenter (1944). J. Ind. Hyg. and Toxicol. 26:269.

7. Friedenwald, J. S., W. F. Hughes, Jr., and H.Hermann ( 1944). Arch. of Oph th.31:279.

8. Carpenter, C. P., and H. F.Smyth.Jr. (1946). A m . J.Ophth. 29: 1363.

9. Draize, J. H., G. Woodard, and H. O. Calvery [ 1944),]. Pharm. find Expt. Therapeu 82:377.

10. Draize, J. H., and E. A. Kelly ( 1952). Proc. of the Sci. Sec. of Toilet Goods Assoc. 17:1.

11. Draize, J. H. ( 1955). Food Drug Cosmetics Law J. 10:722.

12. Draize, J. H. ( 1965). Assoc. of Food and Drug Of ficials of the U. S., Topeka, Kans., 46.

13. Lansteiner, K., and J. Jacobs (1935). J.Exp.Med. 61: 643 .

14. Lansteiner, K., and J. Jacobs (1936). J.Exp. Meal, 64:625.

15. Buehler, E. V. (1965). Arch. Derm.91:171.

16. Baer, R. L., S. Rosenthal, and C.J. Sims [1956). J. Invest. Derm. 27:249.
17. Marzulli,F.N., and H.I.Maibach ( 1970). J. Soc. Cosmet.Chem. $21: 695$.

18. Maguire, H. C., Jr.( 1973). J. Soc. Cosmet.Chem 24: 151.

19. Levine, B. B. ( 1960). J.Exp.Med.112: 1131

20. Salvin, S. B. (1965). Fed. Proc, 24:40.

$21_{4}$ Turk, J. L. (1964). Int. Arch. Allergy 24:191.

22. Biology of the Immune Response ( 1970). P. Abronoff and M. LaVie, eds., McGraw-Hill, N.Y.

23. Textbook of Immunopathology (1976). H. G. Muller-Eberhard,cd., Grune and St rat ton, N. Y..

24. Medical Physiology (1968). V. B. Mountcastle, cd., Mosby, St. Louis.

25. A Textbook of Histology ( 1964). W. Bloom and D. W. Fawcett,eds., Saunders, Philadelphia.

26. Pathology (1971). W. A. D. Anderson, cd., Mosby, St. Louis

27. Nelson, A. A. [ 1955). Food Drug Cosmetic Law J 10:732.

28. Comroe, J. H.,Jr., R. E. Forster II, A. B. Dubois, W. A. Briscoe, and E. Carleson ( 1962). The Lung: Clinical Physiology and Pulmonary Func tion Tests, Yearbook Medical Publ., Chicago.

29. Armstrong, M. L. (1974). Electrocare tiograms. Yearbook MedicalPubl., Chicago.

30. Current Practice of Clinical Electroencephalography (1977). D. W'. Klass and D. D. Daly, eds.. Raven.

31. Goodale, R.H. ( 1965). Clinical Interpretation of Laboratory'Tests, Davis, Philadelphia.

32. Henry, R. J. ( 1974). Clinical Chemistry: Principles and Techniques, Harper Medical. 
33. Amuino, J.S. (1976). Clinical Chemistry: Principles and Procedures, Little.

34. Xintaras, C., and B. L. Johnson (1976). Essays in Toxicology, W. J. Hayes, cd., 7:155, Academic Press, New York

35. Ekel, G. J., and W. H. Teichner (1976), A n Analysis and Critique of Behavioral Toxicology in the U. S. S. R., 13 HEW (NIOSH) Publication No. 77-60, Cincinnati.

36. Behavioral Toxicology (1974). C. Xintaras, B. L. Johnson, and I. DeGroot, eds. DHEW (NIOSH) Publication No. 74-126, Cincinnati.

37. Weiss, B., J. Brozek, H. Hanson, R. C. Leaf, N. K. Mello, and J. M. Spyker (1975). Principles for Evaluating Chemicals in the Environment t. $198 \mathrm{Na}$ tional Academy of Sciences, Washington, D.C.

38. McCa arm, J., and B. M. Ames ( 1976). Ann. N. Y. Acad. Sci. $271: 5$.

39. Bridges, B. A. (1973). Environ. Health Perspect 6:221,

40. Bridges, B.A. (1974).Mut.Res. 26:335

41. Flamm. W. G. ( 1974). Mut. Res. 26:329.

42. Ehrenberg. L. ( 1973). In: Chemical Mu tagens: Principles and Methods for Their Detection, vol. 2. A. Hollaender, ed., Plenum Press, New York.

43. Underbrink, A. E., L. A. Schaierer, and A. H. Sparrow (1973), In: Chemical Mutagens: Principles and Methods for Their Detection, vol. 2, A. Hollaender, cd., Plenum Press, New York.

44. Ames, B. N.. F. D. Lee, and W. E.Durston (1973). Proc. Nat. Acad.Sci., U.S.A. 70:782.

45. Ames, B. N., J. McCann, and E. Yamasaki ( 1975). Mut. Kes. $31: 347$

46. Laishes, B. A., and H.F. Stich (1973). Biochem. Biophys. Res. Commun. 52:827.

47. Brusick. D. J., and V. W. Maver (1973). Env. Health Perspect. 6:83.

48. Ong. 'T-M, and H. V. Malling ( 1975). Mut. Kes. 31: 195 .

49. Mohn. ('., J. Ellenberger, and D. MoGregor ( 1974). Mut.Res. 25: 187.

50. Mortimer, R. K., and T. R. Manney (1971). In Chemical Mutagens: Principles and Methods for Their Detection, vol. 1, A. Hollaender, cd., Plenum Press, New York.

51. IeSernes, F. J., and H. V. Malling ( 1971). In: Chemical Mu tagens. Principles and Methods for Their Detection, vol. 1, A. Hollaender, cd., Plenum Press, New York.

52. Zimmerman F.K. ( 1975). Mut Res. 3 1:71.

53. Gabridge,M.G., A. DeNunzio, and M. S. Legator ( 1969).N(ltur[?68:221,

54. Siebert. D. (1973). Mut.Res.17:307

55. Durston, W. E., and B. N. Ames (1974). Proc. Nat. Acad.Sci. USA $71: 737$.

56. Commoner, B.. A. J. Vithavathil, and J. Henry (1974). Nature 249:850

57. Brew en, J. (3.. P. Nettesheim, and K. P. Jones ( 1970), Mut Res.10:645

58, l.egator. M. S., andII.V.hi; \}lling [ 1971 ). In: Chem. ical Mutagens: Principles and Methods for Their
Detection, vol. 1, A. Hollaender, cd., Plenum Press, New York,

59. Slater, E. E., M. D. Anderson, and H. S. Rosenkranz (1971). Cancer Res. 31:970.

60. Chu, E. H. Y. ( 1971). In: Chemical Mutagens: Principles and Methods for Their Detection, vol. 1, A. Hollaender, ed.. Plenum Press, New York.

61. Kao, F-T, and T. T. Puck (1974). Methods Cell Biol $8: 23$.

62. Cline, D., and J-A F. S. Spector (1975). Mut. Res. 31: 17.

63. Regan, J. D., and R. B. Setlow ( 1973). In: Chemical Mutagens: Principles and Methods for Their Detection, vol. 2, A. Hollaender, cd., Plenum Press, New York.

64. Sega, G, A., J. G. Owens, and R. B. Cumming (1976). Mut. Res. 36: 193

65. Taylor, J. H. ( 1958). Genetics 43:5 15.

66. Allen, J. W., and S. A. Latt (1976). Nature 260:449.

67. Abrahamson, S., and E. B. Lewis (1971). In: Chemical Mutagens: Principles and Methods for Their Detection. vol. 1, A. Hollaender, ed., Plenum Press, New York.

68. Vogel,E.,and B. Leigh ( 1975), Mut. Res. 29:383.

69. Casida.J. E. ( 1969). In:Microsomes and Drug Oxidation. J. R. Fouts and G. J. Mannering, eds., Academic Press. New York.

70, Smith, R. H., and R. (., von Borstel ( 1973). In: Chemical Mutagens: Principles and Methods for Their Detection. vol. 2, A. Hollaender,ed.,Plenum Press, New York.

71. Schmid, W. (1975). Mut. Res. $31: 9$.

72. Leonard, A. ( 1973). In: Chemical Mutagens: Principles and Methods for Their Deter; tion, vol. 2, A. Hollaender, cd., Plenum Press, New York.

73. I.eonard. A. ( 1975). Mut.Res. 31 :291.

74. Russell, 1,. B. ( 1976). [n: Chemical Mutagens: Principles and Methods for Their Detection, vol. 4, A. Hollaender, cd., Plenum Press, New York.

75. Bateman, A. J., and S. S. Epstein ( 1971 ). In: Chemical Mutagens: Principles and Methods for Their Detection, vol. 1, A. Hollaender, cd., Plenum Press, New York.

76. Sontag. J. M., M.P. Page, and U. Saffiotti ( 1976). In: Guidelines for Carcinogen Bioassay in Small Rodents. U.S. Department of Health, Education and Welfare, Public Health Service, National Insti tutes of Health. National Technical In forma$\mathrm{t}$ ion Service PB-264 061, Springfield, $\mathrm{Va}$.

77. Saffiotti. U., and H. Autrup ( 1978). In: In Vitro Carcinogenesis: Guide to th e Literature. Advancesand Laboratory Procedures, U.S. Department of Heal t h, Education and Welfare, Public Health Service, National Institutes of Health. National Technical Information Service PB-281 737, Springfield, Va.

78. Carcinogenicity (1973). In: The Testing of Chemicals for Carcinogenicity. Mu tagenicity, and Teratogenicity. Ministry of Health and Welfare, Canada. 
79. 43 ('FR 37336 ( 1978).

80. Purchase, I. F. H., E. Longstaff, J. Ashby, J. A. Styles. D. Anderson, P. A. Lefevre, and F, R. Westwood (1978). Br.J.Cancer (in press).

81. St vles. J. A. ( 1977), Br.J. Cancer 36:558.

82. Williams, I). J., and B. R. Rabin ( 1971). Nature 232: 102.

83. Bock; F.H., and R. Mund ( 1958), Cancer Research 18:887.

84. Iversen,O.H., and A. Evensen ( 1962). In: Experimen tal Skin Ca rein ogenesis in Mice. Norwegian University Press.

85. Longstaff. E., and F. R. Westwood (1978). Unpublished.

86. DiPaola, J. A., R. L. Nelson, P.J. Donovan, and C. H. Evans (1973). Archs. Pathol 95:380.

87. Epstein, J., R.W.Rosenthal, and R. J. Ess( 1955). Anal.Chem. 27: 1435.

88. Szent-Gyorgyi, A., 1. Isenberg, and S. I. Baird (1960). Proc. Nat.Acud.Sci.U.S.A. 16: 1444.

89. Szent-Gyorgyi, A., and J. McLaughlin (1!361 ). Proc. Not.Acrud.Sci.11.S.A. 47: 1397.
90. McCann, J., and B. N . Ames ( 1976). Proc. Nat. Acad. Sci. U.S.A. 73:950.

91. Heddle, J. A., and W. R. Bruce ( 1977). In: Origins of Human Cancer. Book C. Human Risk Assessment. H. H. Hiatt, J. D. Walson, and J. A. Winsten, eds. Cold Spring Harbor Labor a tory.

92. Teratogenicity (1973). In: The Testing of Chemicalsfor Carcinogenicity. M u tagenicity, and Te Itogenicity. Ministry of Health and Welfare, Canada.

93. Courtney, K. D., andM. Chernoff ( 1974). In: Training Manual for Teratology. ()ffice of Research and Development, U.S. Environmental Protection Agency, Research 'Triangle Park, N.C.

94. Green, E. L. ( 1962). Genetics. N.Y. 47: 1085.

95. Compartment ts, Pools and Spraces i n Medical Physiology ( 1967). P.-Ii. E. Bergnerand (. C. I,ushbaugh,eds. U.S. Atomic Energy Commission, Division of Terhnical In forma t ion, Oak Ridge. T'enn.

96. Purchase, I. ( 1976). Nat ure 264:624. 


\title{
Review and Evaluation of Methods of Determining Risks From Chronic Low- Level Carcinogenic Insult *
}

\author{
by Kenny S. Crump and Marjory D. Masterman
}

\section{BASIC PRINCIPLES AND CONSIDERATIONS}

To aid in determining a proper regulatory action regarding a carcinogen that is present in man's environment, whether it be a feed additive, industrial pollutant, or otherwise, it is helpful to have some knowledge about the number of extra cancers that are likely to be caused by the presence of the carcinogen in the environment. It is also helpful to have some knowledge of the likely change in number of extra cancers that would accompany some projected increase or decrease in the level of human exposure occurring either as a result of regulatory action or inaction. This kind of information is usually impossible to obtain directly from human data. For this reason it is often necessary to use data from animal feeding experiments to estimate human risk. This procedure involves two difficult steps: 1) relating the animal risk at high doses to doses very near to zero and 2) relating the animal risk to risk in humans.

Typically, animal experiments use on the order of 100 animals at each experimental dose. If a particular experimental dose causes a lifetime increase in cancer risk of $1 / 10$, this increase can be measured with a small degree of accuracy using 100 animals. But if the increased cancer risk is less than 1/100 this increase will often not even be detectable by an animal feeding experiment. For example, if the true risk is $1 / 100$ it would require that over 400 animals be tested at that dose in order to be 99-percent sure of detecting any carcinogenic response at all (i. e., for there to be a probability of 0.99 that at least one animal gets cancer). If background or spontaneous carcinogenesis is present even larger numbers of animals will be required, On the other hand, the extra human risk that we may want to estimate resulting from environmental exposure is usually (and hopefully) smaller than $1 / 100$ for any given chemical, perhaps on the order of $1 / 1,000,000$. It is clear that it would not be practical to conduct an experiment with enough animals to measure directly an increase in risk this small.

For these reasons the procedure has been developed of conducting lifetime animal feeding experiments using, in addition to a control dose of zero, several doses at which the projected extra cancer risk may be $1 / 10$ or larger, These highdose data are then used to estimate the extra risk at a dose where the extra risk may be no larger than, say, 1/1,000,000. An equally important variant to this problem is the calculation of the socalled "safe" dose, that is, a dose for which there is some measure of statistical assurance that the extra risk at that dose is no more than, say, $1 / 1,000,000$ These problems are often referred to collectively in the literature as the "low-dose extrapolation problem."

\section{IMPORTANCE OP THE MATHEMATICAL MODEL}

Performing a low-dose extrapolation involves the choice of a mathematical function to model the dose-carcinogenic response relationship and

\footnotetext{
*Excerpt from OTA Working Paper entitled "Assessment of Carcinogenic Risks From PCBS in Food." A complete copy of the paper can be obtained from the National Technical Information Service. (See app. J.)
}

the choice of statistical procedures to apply to the mathematical function. The choice for this mathematical function turns out to be extremely crucial to the outcome of low-dose risk estimation. If the assumed relationship between tumor occurrence and dose does not apply in the regions to which the extrapolation is being made, a serious over- 
estimate of the "safe" dose may result (Mantel and Bryan, 1961, p. 458). Chand and Heel (1974) compared five standard dose-response models and observed that they could differ by many orders of magnitude at low dose levels for which extra risks are on the order of 1/100,000,000.

It might be supposed that it should be possible to discriminate among the various potential doseresponse functions on the basis of experimental data but, unfortunately, two different dose-response functions can often fit experimental data equally well but still differ by several orders of magnitude at very low doses. Moreover, even if a particular dose-response function were to give a significantly better fit to data than several others this would still not furnish assurance that this function would necessarily correlate in any way with the true dose response at very low doses where it is not feasible to measure the true extra risk directly. As a consequence of the great disparity of dose-response functions at low doses it is imperative that the dose-response function be selected, neither arbitrarily nor solely on the basis of how well it can be made to fit experimental data, but, insofar as is possible, it should reflect known or at least plausible information regarding the biological mechanisms through which a chemical induces or promotes cancer.

\section{WHAT SHAPE SHOULD BE EXPECTED FOR THE DOSE-RESPONSE CURVE AT LOW DOSES?}

Tumors of so many different types arise in such a diversity of different tissues, their etiology is so little understood, and the agents that cause tumors affect a subject in such diverse ways, that it might seem that no general conclusions can be drawn. However, for a certain broad class of "directly acting" chemical carcinogens the range of uncertainty associated with the shape of the doseresponse curve at low doses can be greatly narrowed. As used in this paper, the term "directly acting carcinogen" encompasses (Guess, Crump, and Pete, 1977) carcinogenic agents for which either the agent itself or a metabolize acts directly at the cellular level and produces a heritable change that eventually leads to the formation of a tumor. Carcinogens that are carcinogenic by reason of their mutagenicity should fall into the category of "directly acting carcinogens. " Accordingly, carcinogens that test positively using the Ames mutagenicity screening test for carcinogenicity are very likely to be directly acting (see McCann and Ames, 1976). In a recent study (McCann, Choi, Yamasaki, and Ames, 1975) in which about 300 carcinogens and noncarcinogens were tested using the Ames test, 90 percent (157 out of 175) of the carcinogens were mutagenic including almost all of the known human carcinogens. This indicates that the class of directly acting carcinogens may encompass most of the known carcinogens,

A partial solution to the low-dose extrapolation problem for the case of directly acting chemical carcinogens has been given in Peto (1977), Crump, Heel, Langley and Peto (1976), and Guess, et al. (1977). The key result is that, at least as long as background carcinogenesis is present, we should expect the dose-response curve not to be absolute- ly flat at zero dose. What this means is simply that when risk is plotted against dose response on ordinary linear scales, the tangent line to the doseresponse curve at zero dose should have a positive slope. When a dose-response function has this property we will say it is linear at low dose. This simple property can have far-reaching consequences on low-dose extrapolation. For example, consider the two potential dose-response functions:

\section{1. $0.1\left[(99 / 999) d+(900 / 999) d^{2}\right]$ and}

2. $0.1 \mathrm{~d}^{2}$ for the dose interval $0 \leq \mathrm{d} \leq 3$.

Both of the curves give a risk of $1 / 10$ at a dose of $d$ $=1$ and are practically indistinguishable at higher doses. However, at a dose of $d=1 / 1,000$ : 1) predicts a risk of $1 / 100,000$ and 2) predicts a risk of $1 / 10,000,000$, a difference of two orders of magnitude. We note also that 1 ) has a tangent line with a positive slope at $\mathrm{d}=\mathrm{O}$ whereas 2) does not.

One explanation of why the dose-response function should be linear at low dose when background is present may be found in Crump, et al. (1976) and Peto (1977) and will be briefly outlined here. When background carcinogenesis is present, the cellular mechanism through which the test agent produces cancer should already be operative in producing background tumors. When this is true the effect of the test agent is to add to an already ongoing process. The result of this additive effect is illustrated in figure $\mathrm{D}-1$. The dose-response curve is for all tumors produced through the mechanism through which the test agent acts. Background carcinogenesis is allowed for in the figure by an effective background dose $d_{0}$. We see that, in this case, the added risk caused by a dose $d$ of the test agent should be ex- 
pected to increase approximately linearly near $d$ $=\mathrm{O}$ (i.e., the tangent line at $\mathrm{d}=\mathrm{O}$ will have a positive slope). Implicitly assumed by the way figure D-1 is drawn is the fact that an added dose of a carcinogen acting through this mechanism does not produce a smaller risk, If background carcinogenesis is allowed for as in figure D-1 by positing an effective background dose $d_{0}$ that is estimated from the data, then the wide range of risks obtained using different models effectively disappears (Pete, 1977). We note that the existence of a tangent line with a positive slope at zero dose does not, in itself, imply any lower bound for extra risk at low doses since the slope of the tangent line could possibly be very small.

The evidence given above for a positive slope to the dose-response function at zero dose applies particularly to the case in which background carcinogenesis is operative. This does not imply that we expect the dose-response curve not to be linear at low dose in the absence of background carcinogenesis. For example, the multistage models of cancer (Armitage and Doll, 1961) are a fairly broad class of models in which it is assumed that

\section{Figure D-1 .- Illustration of Why the Dose" Response Curve Should Be Linear at Low Dose in the Presence of Background Carcinogenesis. $d_{o}$ Is the Effective Background Dose and $d$ Is the Dose of the Carcinogen of Interest}

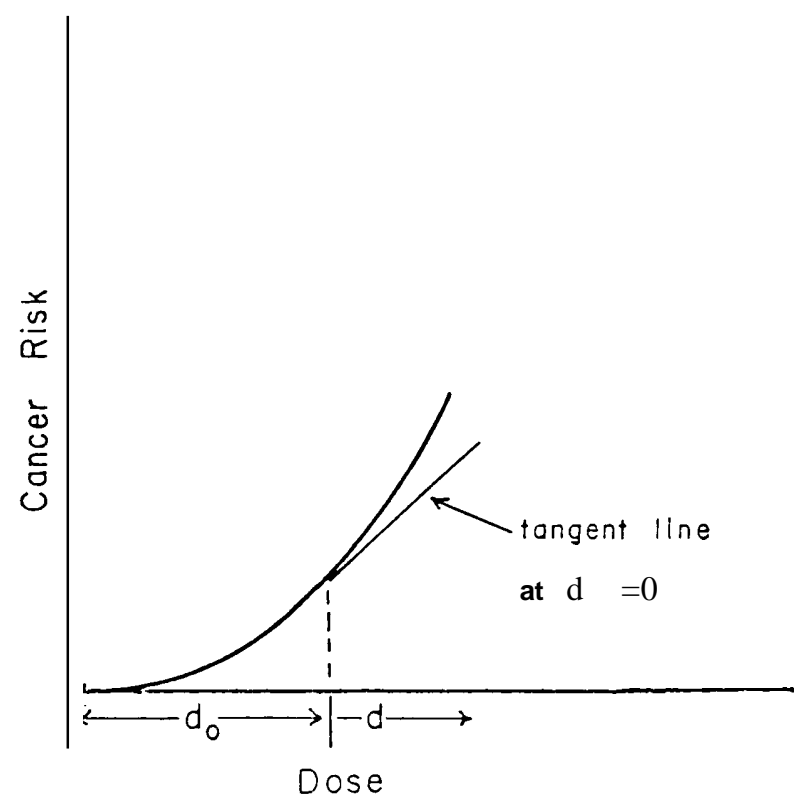

a number of events are required to occur at the cellular level to initiate cancer. Although all models in this class are linear at low dose provided background carcinogenesis is present, a sizable subclass of them are linear at low dose in the absence of background carcinogenesis (Crump, et al., 1976).

Watson (1977) has recently proposed a more specialized model for cancer induction and promotion based on reversible epigenetic cellular changes. Watson concludes that his model supports the low-dose-linearity hypothesis and states "as suggested by a different argument of Crump, et al. ... it is reasonable to assess the risk due to an additional carcinogen at low constant dosage by a linear relation. "

The evidence for low-dose linearity given above applies mainly to directly acting carcinogens. An indirectly acting carcinogen might be one that causes some gross physiological change such as suppression of ovulation that could predispose the subject to cancer. For such carcinogens the shape of the dose-response curve at low dose is highly speculative. There could possibly be a threshold dose below which the agent has no carcinogenic effect at all on an individual, However even if a threshold mechanism is operative, there is likely to be considerable variation in individual thresholds in a large population. Consequently the dose-response curve for the entire population could still exhibit a linear trend at risks as low as $1 / 1,000,000$ or lower.

The effects of metabolic activation and detoxification on carcinogenic dose response have been recently considered by Cornfield (1977) through a kinetic model that encompasses free toxic substance, metabolize, deactivator, and the interactions of these substances. Only a steady-state situation is studied in that variation over time of the concentrations of these agents is not considered. The model predicts a threshold dose below which there is no carcinogenic risk under the assumption that the deactivator is 100-percent efficient in deactivating the carcinogen. However, in a naturally occurring process it is likely that deactivation would not be perfect and would be less than 100-percent effective in always combining with 100 percent of the carcinogen before an amount of the active metabolize reaches a cancer target site, Any of a number of modifications to the model to allow for nonperfect deactivation would rule out a threshold and would lead directly to a model for which carcinogenic response varies linearly with dose at low doses, Cornfield's own modification of perfect deactivation, that of al- 
lowing the deactivating reaction to be reversible, leads, as Cornfield points out, to a model which is linear at low dose. This occurs regardless of how slowly the reverse reaction takes place, as long as the possibility is not eliminated entirely. Furthermore, even in the extremely unlikely case of perfect deactivation, an otherwise realistic model should still imply low-dose linearity since the theoretical time required for perfect deactivation would not be zero and would likely be infinite.

For most, perhaps all, carcinogens, the mechanisms through which cancer is produced are not sufficiently understood so that the shape of the carcinogenic-response curve can be theoretically predicted with certainty. As pointed out earlier, neither can experiments of sufficient size be conducted that would permit direct experimental investigation of the dose-response curve at low dose. We have noted that there are plausible arguments that the dose-response curve is linear at low dose for many carcinogens. On the other hand, this author knows of no serious proposal of a mechanism that would lead to a more conservative dose-response relationship such as the risk varying approximately as the square root of dose at low dose. In view of these uncertainties it would seem reasonable to base estimates of added risk of cancer on a mathematical model that encompasses low-dose linearity unless, of course, the mechanism through which the carcinogen operates is sufficiently understood so that low-dose linearity can be conclusively ruled out. Once the principle of low-dose linearity is accepted the problem of estimation of risks at low dose is nearly solved. This is because the disagreement between the upper statistical confidence bounds on risk at low doses based on a model that incorporates low-dose linearity and one that does not is typically several orders of magnitude whereas the corresponding disagreement between two reasonable models both of which incorporate low-dose linearity is usually much less than this.

\section{SPECIFIC METHODS FOR LOW-DOSE RISK ESTIMATION}

$$
\text { Mantel= Bryan }
$$

The Mantel-Bryan procedure as originally proposed (Mantel and Bryan, 1961) and "improved" (Mantel, Bohidar, Brown, Ciminera, and Tukey, 1975) is for the purpose of conservatively choosing a "safe" dose of a carcinogen, a "safe" dose being defined as one for which it can be expected that, with a given level of statistical assurance (e.g., 99 percent), the true dose producing a preassigned "safe" level (e. g., 1/1,000,000) of risk will lie above the "safe" dose. In the MantelBryan procedure the mathematical model used for the dose-response model is the probit function:

$$
P(d)=C+(1-C) J-\infty \quad\{<\pi)^{-\cdots} \exp \{-x<<\} d x
$$

where $d$ represents the dose of the carcinogen and $\mathrm{P}(\mathrm{d})$ represents the probability of a cancerous response in an animal subjected to a dose $d$. The parameters in the model are an intercept parameter $\mathrm{a}$, a probit slope parameter $\mathrm{b}$, and $\mathrm{C}$, which represents the probability of a response in untreated animals. The parameter $b$ is not estimated from the data but rather is arbitrarily set equal to 1. This choice is stated as being conservative (Mantel and Bryan, 1961), the argument for this being that typical dose data exhibit a probit slope in the experimentally observable region above 1 percent incidence that is greater than one, In Mantel and Schneiderman (1975) it was observed that a set of DES data (Gass, Coats, and Graham, 1964, C3H females) exhibited a probit slope of one-half, but the general use of a probit slope of $b$ $=1$ was still suggested.

With the probit slope parameter fixed at $\mathrm{b}=1$ the remaining parameters, $\mathrm{a}$ and $\mathrm{C}$, are estimated from the data and then adjusted so as to produce a higher level of risk at a given dose that corresponds to an upper 99-percent statistical limit on the true risk at a given dose. The safe dose is then determined to be the one producing a given low risk (e. g., 1/1,000,000) based on the adjusted values of a and $\mathrm{C}$.

As pointed out in Mantel, et al. (1975), the Mantel-Bryan procedure rewards larger and better experiments in that the more evidence there is of safety, the higher the calculated safe dose will be. However this advantage should be shared by any extrapolation method that uses reasonable statistical procedures.

Some have considered the Mantel-Bryan procedure to be too conservative (Federal Register, vol. 42,1977 , p. 10419) in that it involves three conservative choices (99-percent statistical assurance, lifetime risk of $1 / 1,000,000$, and probit slope set equal to 1) and that any one of these assumptions alone could provide adequate protection to the public. The first two of these choices are regulatory decisions that would have to be made with any extrapolation procedure. However, the arbi- 
trary selection for the slope parameter seems to be peculiar to the Mantel-Bryan procedure. To investigate its effect upon the extrapolation procedure a typical fit is presented in figure D-2 (from Crump, 1977b) of the Mantel-Bryan probit curve (equation 1) to experimental carcinogenicity data when the probit slope parameter is fixed at $b=1$. As can be readily seen the probit curve typically provides a very poor fit, curving downward even when the trend of the data is toward an increasingly upward curvature. This typically bad fit to data of the probit curve raises serious questions regarding the validity of statistical procedures associated with the Mantel-Bryan method (see Salsburg, 1977; and Crump, 1977b).

On the other hand, the Mantel-Bryan procedure utilizing the choice $b=1$ was put forth as conservative procedure and it gives that appearance in figure D-1 since the probit curve appears to lie far above the trend of the data at the lowest doses. However as mentioned earlier and also pointed out by Mantel (Mantel and Bryan, 1961, p. 458) a procedure may, while appearing conservative at experimental dose levels, at the same time seriously overestimate the "safe" dose (i. e., be seriously anticonservative) if the assumed dose-

Figure D-2.-Typical Fit of Mantel” Bryan Curve to Experimental Data (From Crump, 1977b)

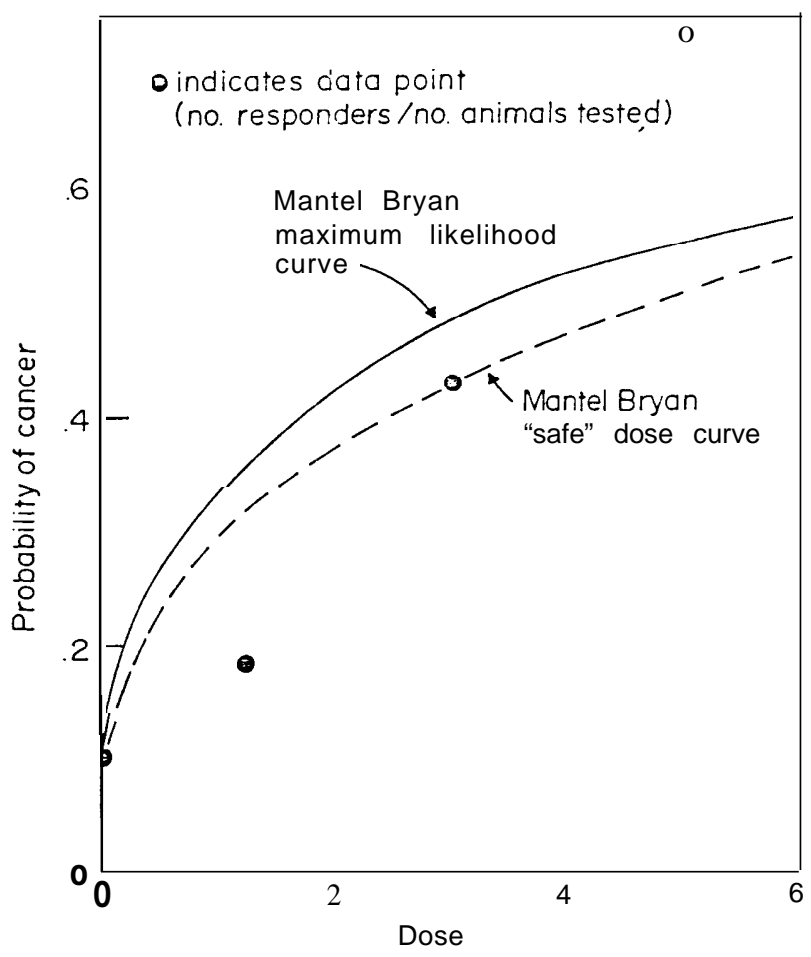

response relationship does not apply at the low risk levels to which extrapolation is 'being made. Thus, before the degree of conservation can be evaluated for any procedure, the properties of the dose-response curve at very low doses must be evaluated,

As described earlier, there are strong arguments that indicate the dose-response curve should be "linear at low dose" particularly for directly acting carcinogens in the presence of background carcinogenesis. This has led Peto (1974) to recommend extrapolation procedures using only dose-response functions from a class containing only dose-response functions that are linear at low dose, At the very least, however, it would seem prudent not to go to the opposite extreme and use a dose-response function that rules out linearity at low dose by assumption, However, the Mantel-Bryan procedure, through its use of the probit curve (equation 1) rules out linearity at low dose in favor of a "flatness property" (see Hartley and Sielken, 1977; Mantel, 1977; and Crump, 1977b) at low dose which is anticonservative to the extreme. This property implies that mathematical derivatives of all orders of the probit curve approach zero (through positive values) as the dose approaches zero. This unusual property is more often discussed within the context of mathematical oddities rather than in connection with a scientific investigation. It implies that if the true dose-response curve comes from an extremely broad class of functions known as analytic functions and which pervade scientific applications of mathematics, then the probit curve will eventually underestimate the true risk at low doses. Furthermore, at low enough doses, the degree to which the risk will be underestimated will be arbitrarily large (i.e., the ratio of the true risk to the probit estimate will grow arbitrarily large).

It was emphasized earlier that it is important when extrapolating to low doses for the assumed dose-response function to incorporate known or at least plausible facts about the mechanisms of carcinogenesis. In neither the original paper (Mantel and Bryan, 1961) nor in the paper outlining the improved version is biological justification given for the selection of a curve having the above described "flatness property." It should be mentioned at this point that the incorporation of background carcinogenesis into the Mantel-Bryan probit model (equation 1) using the parameter $\mathrm{C}$ implies that the mechanism through which the test carcinogen produces cancer is independent of the mechanisms through which all of the back- 
ground cancers are produced (Crump, et al., 1976). In keeping with the discussion in the last section it would seem more proper to incorporate background into the probit model by positing an effective background dose $d_{0}$ which adds to the dose $\mathrm{d}$ of the test carcinogen. If background is incorporated in this way the probit curve no longer has the "flatness property" and becomes linear at low dose (Guess, et al., 1977). In fact. with background incorporated in this way, the probit curve assumes a shape similar to the one-hit model, sometimes referred to as the most conservative of all procedures (e.g., Mantel, 1977).

Even though the "flatness property" implies the probit curve should at suitably low doses be anticonservative to the extreme, the "flatness" property holds only for doses approaching zero and the feature of arbitrarily fixing the probit slope at 1 mitigates the anticonservativeness implied by the "flatness" property at any given low dose (although the property itself will hold for all choices of the parameters $a, b, C)$, The cancer risks that are typically extrapolated to are in the risk ranges $1 / 10,000$ to $1 / 100,000,000$. We will examine the outcome of Mantel-Bryan extrapolations to these risk levels in a later section when we compare them with extrapolations based on the multistage model.

\section{Linear Extrapolation}

The technique for linear extrapolation was recommended by Heel, Gaylor, Kirschstein, Safiotti, and Schneiderman (1975) for use on an interim basis until better procedures could be developed. The procedure is straightforward and is based on an assumed linear relationship between dose and response at low dose. The procedure utilizes only the data for the group of control animals and a single other dose group, usually either the highest dose that elicits no response or else the lowest dose that elicits some response. In the case there are no cancers in the control animals the "safe" dose, based on a maximum risk of $1 / 1,000,000$ and 99-percent statistical assurance, is calculated as follows: An upper 99-percent confidence bound is calculated for the cancer risk in the dose group of animals. From this risk and dose one extrapolates back toward zero dose and zero risk using a straight line relationship. The dose corresponding to a risk of $1 / 1,000,000$ on this straight line is taken to be the "safe" dose. If there are cancers in the control animals this procedure is modified to allow for the statistical treatment of the response in the control group while retaining the straight line relationship. When data at other experimental doses are available this method of linear extrapolation has the obvious shortcoming of not fully utilizing the available data.

A linear dose-response curve is linear at low dose but the converse is not necessarily true. A curve can be linear at low dose and still have a high degree of nonlinearity at higher doses.

Linear extrapolation is viewed by some as a very conservative procedure. For example, comments were made during the decision on which extrapolation procedure to incorporate into the SOM document to the effect that linear extrapolation is the most conservative of all procedures. Crump, et al. (1976) examined the extent of the conservatism of a linear dose-response function when compared with a multistage dose-response model (Armitage and Doll, 1961). The multistage model assumes that a cell must go through a number of different stages before cancer is initiated in that cell and the model can encompass a high degree of nonlinearity. It was determined that the maximum possible degree of conservatism of a linear model relative to a multistage model depended rather heavily on the incidence at the experimental dose relative to the background incidence. (This is consistent with the general relationship between background carcinogenesis and linearity at low dose as discussed earlier. ) For example, when the incidence at the experimental dose is four times the incidence at zero dose the extra incidence at low dose derived from the linear dose response differs from the incidence derived from the multistage model by, at most, less than a factor of 2.5 regardless of the number of stages in the multistage process. Thus, when background carcinogenesis is present, the linear dose-response curve is not overly conservative relative to the multistage dose-response curve. In fact the linear dose-response curve is anticonservative when compared to the one-stage or onehit models.

Linear extrapolation has long been proposed for use in radiation carcinogenesis (see Brown, 1976, for a review of the relevant reports). The BIER (1972) report on radiation risks from the National Academy of Science recommended linear extrapolation as a "best estimate" approach as opposed to a conservative approach. Brown reviewed arguments both for and against linearity and concluded that "linear extrapolation of human data from high dose of low LET radiation cannot be said to overestimate the risk at low 
doses. In fact, there is some doubt as to whether the risk is not underestimated.

Certainly much remains to be learned about both radiation and chemical carcinogenesis. However, if both radiation and chemicals cause cancer through similar mechanisms then it should be expected that there would also be similarities between the respective carcinogenesis dose-response functions. Direct damage to DNA by the carcinogenic agent has been implicated as one cancer-initiating mechanism for both radiation and chemicals (Brown, 1976; and McCann and Ames, 1976). Thus, the findings related to the potential linearity of the dose-response function for radiation has implications for chemical carcinogens as well, particularly for "directly acting" carcinogens.

\section{Extrapolation Methods Based on the Multistage Model}

Two methods of low-dose extrapolation which are alternatives to the Mantel-Bryan or linear procedures have recently been proposed independently by Guess, Crump, and Deal (Guess and Crump, 1976, 1978; and Crump, Guess, and Deal, 1977) and Hartley and Sielkin (1977). Both of these methods utilize a multistage dose response function of the form:

$$
P(d)=1-\exp \left\{-\left(q_{0}+q_{1} d+q_{2} d^{2}+\ldots+q_{k} d^{k}\right)\right\}(2)
$$

where $q_{0}, q_{1}, \ldots, q_{k}$ are all non-negative parameters to be estimated from the data. This dose-response function is general enough to yield a considerably wide range of responses at low dose. On the one hand, if $\mathrm{q}_{1}>0$ and $\mathrm{q},=0$ for $\mathrm{i} \geq 2$ the doseresponse function (equation 2) becomes the onestage model which yields risks at low doses comparable to what would be obtained with linear extrapolation, On the other hand, the model can produce risks even as low as the probit curve (equation 1) down to any fixed positive low dose. Thus this model is capable of fitting both highly linear and highly nonlinear dose-response relations. Since the model has the property of "linear at low dose" if $\mathrm{q}_{1}>0$ and does not have this property if $\mathrm{q}_{1}=\mathrm{O}$, use of this model does away with having to make the arbitrary but crucial decision of having to either assume linear at low dose as in linear extrapolation. Thus "safe" doses computed using this model should provide a more realistic measure of the true uncertainty of low-dose extrapolation than would "safe" doses based on either an assumed linear curve shape or an assumed highly nonlinear curve shape.
The dose-response relation (equation 2) contains all of the Armitage and Doll (1961) multistage dose-response models as special cases but also contains curves that are much flatter at low dose than any of the multistage curves.

The two extrapolation procedures based on equation 2 have some features that are different. When computing "most likely" estimates the procedure of Guess, Crump, and Deal uses an infinite dimensional maximization procedure so that it is not necessary to specify a value of $k$, the degree of the polynomial in equation 2 . However the two methods differ chiefly in the way the statistical confidence intervals are computed. There have not yet been sufficient comparative calculations made to determine how safe doses may differ using the two approaches, Mantel (1977) has made a critical review of the statistical procedure used by Hartley and Sielkin for calculating the "safe' dose.

Both the Hartley and Sielkin and the Guess, Crump, and Deal (as extended by Daffer et al., 1979) procedures can utilize times at which cancer is detected in the experimental animals rather than just the dichotomous information of whether or not an animal contracted cancer before it died of some other cause or before the termination of the experiment, The utilization of time data in low-dose extrapolation is important for at least two reasons: 1) the age at which cancers occur should be important in assessing the magnitude of the harmful effect of a carcinogen on man (e.g., cancers that occur early in life should be viewed as more serious than those that occur in extreme old age); and 2) in many animal carcinogenicity experiments the response data at the highest doses lies below the trend of the lower dose data, This sometimes appears to be due to the fact that at the highest doses some of the animals are being poisoned by the chemical before they have a chance to develop cancer, When this occurs the high-dose data is often just deleted from the analysis. However if the times at which the animals die are properly used the high-dose data might not appear anomalous. More research needs to be done on the proper utilization of animal time of death data to assess the harmful effects of chemicals to man.

\section{Comparisons Between Mantel-Bryan and Multistage Extrapolations}

To compare low-dose extrapolations using the Mantel-Bryan probit model (equation 1) with 
those using the multistage model (equation 2) we present figure D-3 based on the same data as figure D-2. In this figure, the Mantel-Bryan "safe" dose is plotted on a log-log scale as well as both the multistage "most likely" curve and the "safe" dose based on (equation 2) and computed as outlined in Crump, et al. (1977). A 99-percent statistical assurance was used for both "safe" dose curves. We note that the Mantel-Bryan "safe" dose lies above the multistage safe dose curve at values of added risk below $5 \times 10^{-4}$. The MantelBryan "safe" dose curve lies above the multistage "safe" dose curve by a factor greater than 20 for an added risk of $10^{-6}$ and by a factor greater than 300 for an added risk of $10^{-8}$. Because of the "flatness" property of the Mantel-Bryan probit function (equation 1) described earlier, the Mantel-Bryan "safe" dose curve will lie above the multistage safe dose curve by arbitrarily large factors at extreme low doses. Guess, et al. (1977) have compared the Mantel-Bryan "safe" dose curves to the multistage "safe" dose curves and found this to be a typical situation. Thus it is clear that if the true dose-response curve could be similar to the multistage dose-response function (equation 1) then the Mantel-Bryan procedure could not be justifiably called conservative (see

\section{Figure D-3.-Comparisons of "Safe" Doses Computed From the Mantel" Bryan Procedure and From a Procedure Based On the Multistage Model (From Crump, 1977b)}

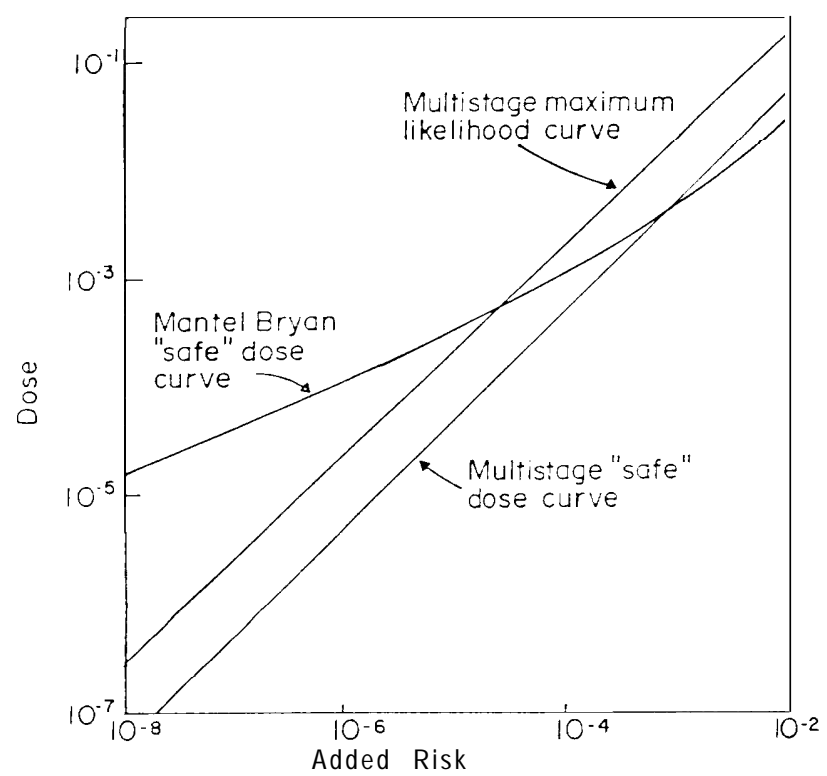

also Crump, 1977 for further discussion of this point.) On the other hand, we have seen that there are quite plausible arguments for the true doseresponse curve to have the same shape at low dose (linear) as the estimated multistage curve.

We note that both the multistage "safe" dose curve and "most likely" curve have a slope 1 in figure D-3 as plotted on the log-log scales which is equivalent to the curves being linear at low dose. The fact that the "most likely" curve has slope 1 is due to the fact that with this particular data set the linear coefficient $q_{1}$ in equation 2 will always be linear at low dose regardless of whether or not the linear coefficient $\mathrm{q}_{1}$ is estimated to be positive. This property should be shared by any valid statistical procedure based on a dose-response function that does not rule out linearity at low dose by assumption as Mantel-Bryan does. Just as it is not possible to prove statistically the existence of a threshold, it is likewise not possible to rule out the possibility that the true dose-response curve is linear at low dose on the basis of statistical analysis. (See Guess, et al. (1977) for a thorough discussion of this important point.) The MantelBryan obtains "safe" dose estimates which are considerably higher than those obtained using the multistage model because it assumes away linearity at low dose, an assumption that we have seen is probably unwarranted for that majority of carcinogens which are classified as "directly acting" carcinogens.

Since extrapolation based on a model such as the multistage model (equation 2) must always be linear at low dose, the question arises as to how different the result will be from simple linear extrapolation. For some data the difference will be minimal. For example, for the data upon which figure D-2 is based, "safe doses" computed using the multistage model are almost identical with *'safe" doses based on linear extrapolation. For some data sets, however, the difference could be considerable. For example, with the Gass, et al. (1964) DES using the C3H female mice, the "safe" dose based upon linear extrapolation is lower than the "safe" dose based on the multistage model by a factor of about five and there are other data sets where this difference is greater than an order of magnitude.

\section{The Gamma Multihit Carcinogenesis Dose-Response Model}

Rai and Van Ryzin (1978) have proposed basing risk estimation on the gamma multihit model: 


$$
\begin{aligned}
& P(d)=C+(1-C) \int_{0}^{\lambda^{d}} \frac{1 j^{k \cdot 1} \cdot e^{u}}{(k-1) !} d u \quad k \geq 0 \\
& =C+(1-C)\left\{1 \cdot e^{-\lambda d}\left[1+\lambda d+\frac{(\lambda d)^{2}}{2 !}+\ldots+\frac{(\lambda d)^{k-1}}{(k-1) !}\right]\right\}
\end{aligned}
$$

where $P(d)$ is the lifetime probability of cancer in a tissue when subjected to a constant dose rate, d, of the carcinogen. This model is obtained by assuming that cancer due to the carcinogen occurs randomly according to a Poisson distribution. The manner in which background carcinogenesis is incorporated into the model is equivalent to assuming that the event "cancer occurs due to the action of the carcinogen" is independent of the event "cancer occurs spontaneously," This assumption would not apply, for example, to processes in which the effect of the carcinogen is to speed up the rate at which the "spontaneous" events occur which lead to the background cancers. At low dose rates, the response is approximately given by:

$$
P(d) \cong \lambda^{k} d^{k} / k !
$$

Consequently, this dose response model is linear at low dose rates when and only when $\mathrm{k}=1$. Rai and Van Ryzin calculate confidence intervals for added risk at a given dose and for the dose producing a fixed added risk using asymptotic maximum likelihood theory. Although in the theoretical development $\mathrm{k}$ must be an integer, in the applications $\mathrm{k}$ is allowed to assume any positive value.

Lower statistical confidence limits on the dose producing a given low amount of extra risk ("virtually safe dose" or "VSD") computed using this procedure can be compared with those computed from the multistage model (equation 2) by considering two general classes of data.

If the data exhibit a general downward curvature as illustrated in figure D-4a, lower confidence limits on a VSD computed from the gamma multihit model (equation 3) should generally be less than or equal to corresponding lower confidence limits computed from the multistage model (equation 2). In certain instances the gamma multihit lower limits could be much less than the corresponding multistage lower limits. This could occur when the data is consistent with $\mathrm{k}<1$ in the gamma multihit model (presumably corresponding to a fraction of a hit).

On the other hand, if the data exhibit a general upward curvature as illustrated in figure D-4b the reverse situation will hold; gamma multihit lower confidence limits on VSDs will generally be greater than or equal to corresponding multistage limits, Gamma multihit limits will not in general share the low-dose linearity of multistage limits by orders of magnitude at low doses. The reason these large differences can occur is as follows. The multistage family of models contains members which are simultaneously linear at low dose and exhibit upward curvature at moderate doses. For example, the particular multistage model:

$$
1-\mathrm{e}^{-\left(\mathrm{q}, d+q_{2} d^{2}\right)} \quad \mathrm{q}_{1}, \mathrm{q}^{2}>0
$$

is linear at low dose since $\mathbf{q}_{i}>0$ and still can exhibit upward curvature at moderate doses since $\mathbf{q}_{2}>0$. This means there are dose-response curves in the multistage class which are both linear at low dose and can adequately describe data of the type exemplified by figure D-4b. On the other hand, this will generally not be true of gamma multihit models. All dose-response curves in the

\section{Figure D-4a.- Example of Data Exhibiting Downward Curvature}

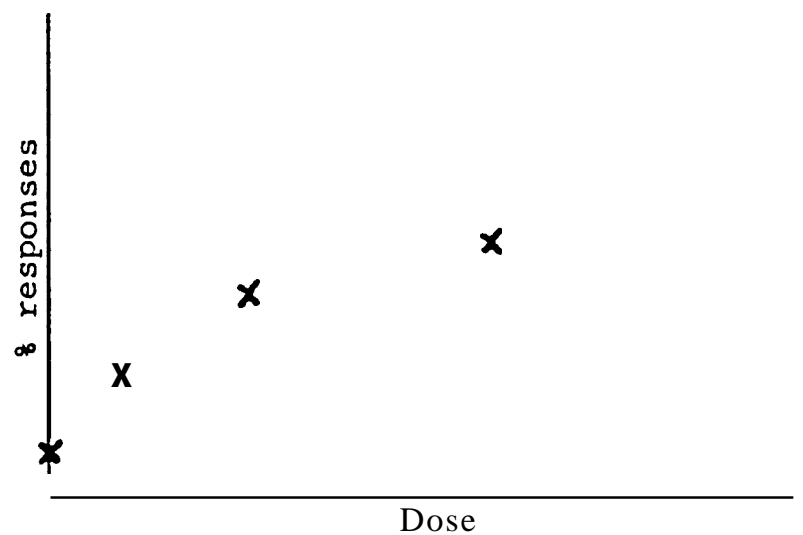

Dose

Figure D-4b._Example of Data Exhibiting Upward Curvature

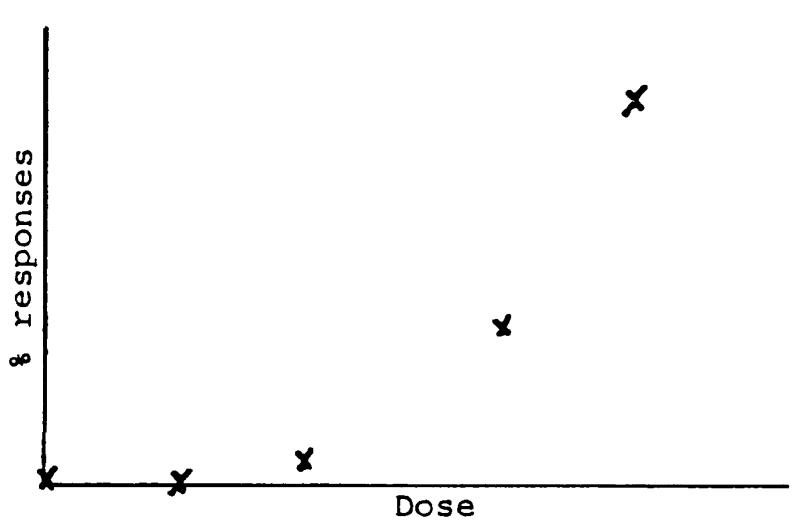


gamma multihit class that are linear at low dose must exhibit downward curvature and consequently would generally not be consistent with the data in figure D-4b. When this is true gamma multihit lower confidence limits on VSDs will be sublinear (e.g., quadratic) at low dose and consequently much larger than the multistage confidence limits.

Confidence limits based on the gamma multihit model will be approximately correct whenever this model is the correct model, A similar statement could be made for the probit model, multistage model, or any other model to which reasonable statistical methods are applied. However, there may be considerable uncertainty as to what the true model may be in a particular situation. The multistage model not only reflects some reasonable assumptions regarding the carcinogenic process which dovetail nicely with epidemiological data for many cancers (Pete, 1977 b), but it also reflects some of the uncertainty with regard to the true model by virtue of encompassing a relatively large class of dose-response functions, For example, as noted earlier, the multistage class contains dose-response functions which are linear at low dose and also exhibit upward curvature at moderate doses. On the other hand, the gamma multihit class is more restrictive at this point in that it does not permit such behavior. Is this extra restrictiveness of the gamma multihit model justified? To help answer this question, consider the following modification to this model. Suppose that the hits (phenomenological events which are required to occur in a tissue in order that a cancer appear) can possibly occur spontaneously in the absence of the carcinogen. Part of the effect of the carcinogen could then be to speed up the rates at which the spontaneous hits are occurring. For example, if one of the "hits" is in incorrect base substitution in DNA during mitosis, the carcinogen could speed up the rate at which these "hits" are occurring in individual cells by providing more aberrant bases for use in such an incorrect substitution. With this modification to the model, the gamma multihit lower confidence limits on VSDs will no longer be sublinear at low dose and should be at least as small as corresponding limits calculated from the multistage model (equation 2). Thus, in order to obtain sublinear lower confidence limits on VSDs with the gamma multihit model a modification such as the one described above must be ruled out, not on the basis of data, but by assumption.

By way of summary, confidence limits based on the multistage model will always be linear at low dose. Confidence limits based upon the gamma multihit model may be either "super linear" (corresponding to $\mathrm{k}<1$ ) or "sublinear" (corresponding to $\mathrm{k}>1$ ). Superlinearity is achieved by making the model too broad in that a fraction of a hit is allowed which has no biological basis. On the other hand, sublinearity is achieved by making the model possibly too restrictive in that models which are reasonable from a biological viewpoint are ruled out by assumption.

In addition to questions as to the appropriateness of the gamma model, there is also a very significant difficulty with the statistical procedure applied to the model in the Food Safety Council (1978) report. This difficulty is illustrated in table D-1. Here are listed lower confidence limits for VSDs for the gamma multihit model which were taken from table D-1 in Food Safety Council (1978). For each of the 14 data sets a goodness-offit test was conducted for compatibility of the data to the subclass of gamma models for which $\mathrm{k}=1$. As indicated by table $\mathrm{D}-1$, the subclass of models with $\mathrm{k}=1$ provided an adequate fit in 10 out of the 14 data sets. For these 10 data sets are listed the VSD predicted by the best fitting gamma model with $\mathrm{k}=1$. The significant point is that in 8 of these 10 cases, the lower confidence limit on the VSD is greater than the VSD from the model with $\mathrm{k}=1$, greater in some cases by enormous factors, e.g., a factor of 3,600 for aflatoxin $\mathrm{B}_{1}$, a factor of 1,000 for diethyl-nitrosamine, and a factor of 18,000 for sodium saccharin. Put another way, in the case of the sodium saccharin data, there is a member in the gamma multihit class which fits the data quite adequately and for which the VSD is less by a factor of 18,000 than the lower confidence limit for the VSD reported in Food Safety Council (1978). This obviously implies there is a serious problem with the computation of the lower confidence limits for the VSD. These lower confidence limits are much too large, even based on assuming the gamma multihit model is the correct model, for these 8 data sets. This problem also exists for the 4 data sets which could not be fit adequately by a model with $\mathrm{k}=1$. This is wellillustrated by the graph in Food Safety Council (1978) for the NTA data. In this graph, the lower 97.5-percent confidence limit for the VSD corresponding to a risk of $10^{-6}$ falls almost directly on top of a data point for which the measured risk is 1/91. Put another way, even though the measured risk at this dose is 1/91, the statistical procedure used indicates, with 97.5-percent assurance, that the risk is no greater than $1 / 1,000,000$.

These difficulties can be overcome by using a different statistical procedure. However, when this is done, the lower confidence limits will be 
Table D-1.-Comparison of Lower 97.5-Percent Confidence Limits on Virtually Safe Doses From Gamma Multihit Model Given in to Virtually Safe Doses From Best Fitting Gamma Multihit Model With $k=1$

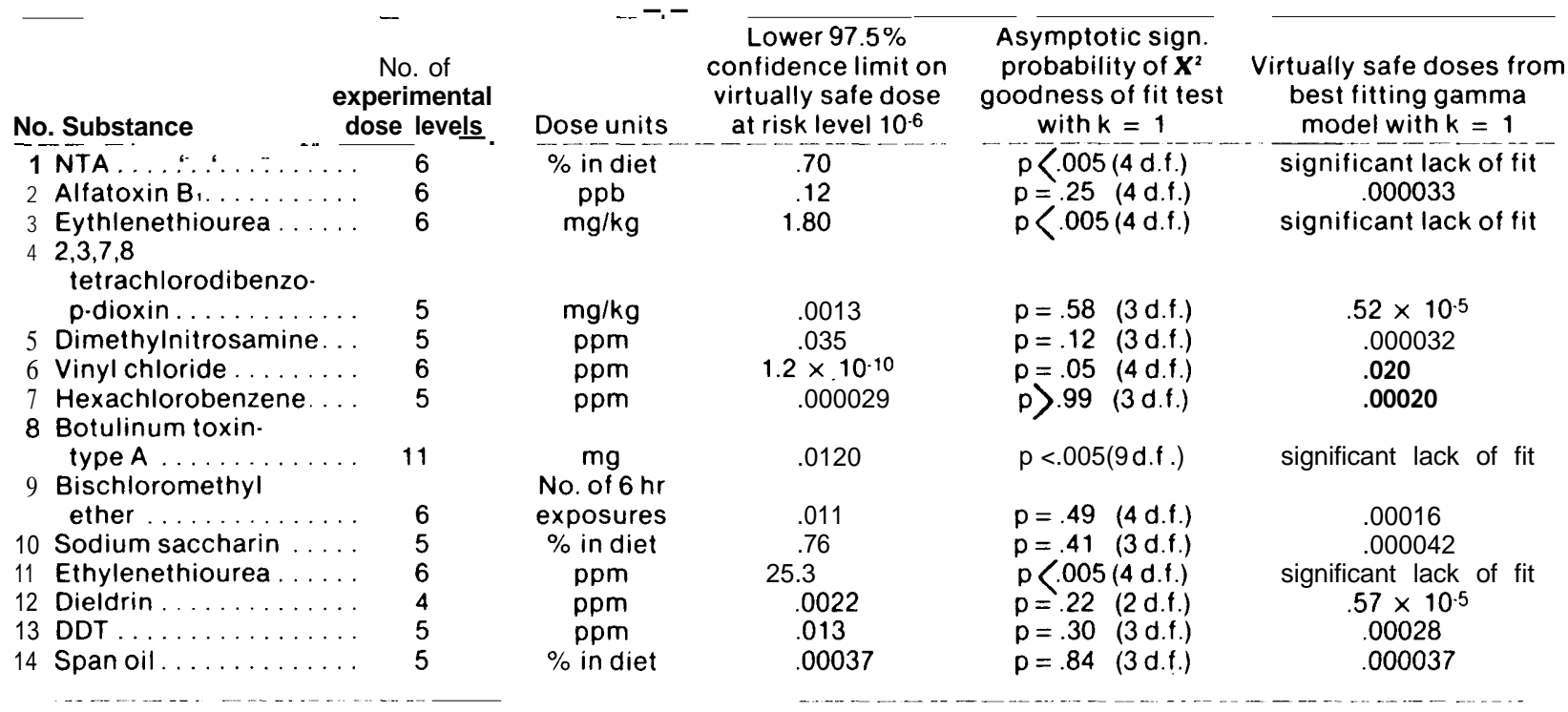

many times smaller than those reported in Food Safety Council (1978). For data sets such as the 10 referred to in table D-1 which are compatible with $\mathrm{k}=1$, lower confidence limits for VSDs must essentially be calculated from a model with $\mathrm{k}<1$ and consequently be smaller than those calculated from the one-hit model. This will lead, in many cases, to super-small confidence limits, as illustrated by the lower confidence limit for vinyl chloride, For those data sets, such as botulinum toxin-type $\mathrm{A}$, which are incompatible with $\mathrm{k}=1$ because of their strong upward curvature, a lower confidence limit for the VSD would still be larger than one computed from the one-hit model. However, the theoretical objections raised earlier to the mathematical form of the gamma model would still apply.

It may not be generally realized that it is uncommon to find data sets which are incompatible with $\mathrm{k}=1$ by reason of strong upward curvature. The four data sets in table D-1 which fall into this category all involved a large number of animals and a number of different experimental dose levels. These four data sets contained from 330 animals distributed over 11 dose levels to 720 animals distributed over 6 dose levels. Most carcinogenicity bioassays are smaller than these, For example, the standard bioassay for a given sex and strain in the National Cancer Institute program seems to be 200 animals distributed over 4 dose levels. For experiments of this size it is more difficult to rule out $k=1$. Consequently it would seem that, for most of the data sets that are likely to result from a large screening program, appropriate statistical procedures applied to the gamma multihit model would yield lower confidence limits for VSDs which are even smaller than those calculated from the one-hit model. 


\section{APPENDIX D REFERENCES}

1. Armitage, P. and R. Doll. Stochastic models for carcinogenesis. In Proceedings of the Fourth Berkeley Symposium on Mathematical Statistics and Probability, vol. 4 (Berkeley and Los Angeles, Calif.: University of California Press, 1961), pp. 19-38.

2. BIER. The effects on populations of exposures to low levels of ionizing radiation, National Academy of Sciences. National Research Council (Washington, D. C., 1972).

3. Brown, J. M. Linearity vs. nonlinearity of dose response for radiation carcinogenesis. Health Physics 31: 231-245, 1976.

4. Chand, N., and D. G. Heel. A comparison of models for determining safe levels of environmental agents. In Reliability and Biometry, F. Prochan and R. J.Serfling (eds.) (Philadelphia, Pa.: SIAM,1974), pp. $382-401$.

5. Cornfield, Jerome. Carcinogenic risk assessment. Science 198:693-699, 1977.

6. Crump, K. S., D. G. Heel, C. H. Langley, and R. Pete. Fundamental carcinogenic processes and their implication for low dose risk assessment. Cancer Research36:2973-2979, 1976.

7. Crump.K.S., H. A. Guess, and K. L. Deal. Confidence intervals and tests of hypotheses concerning dose response relations inferred from animal carcinogenicity data. Biometrics 33:437-451. 1977.

8. Crump, K. S. Response to open query: Theoretical problems in the modified Mantel-Bryan procedure. Biometrics 33:752-755, 1977.

9. Da ffer, P. Z., K. S. Crump, and M. D. Masterman. Asymptotic theory for analyzing dose response survival datawith application to the low-dose extrapolation problem (submitted), 1979.

10. Food Safety Council. Proposed system for food safety assessment. Food and Cosmetic Toxicology (December), 1978.

11. Gass, G. H.. D. Coats, and N. Graham. Carcinogenic dose response curve to oral diethylstilbestrol.J. Natl.CancerInst. 33:971-977, 1964.

12. Guess, H. A. and K. S. Crump. Low-dose rate extrapolation of data from animal carcinogenicity experiments-analysis of a new statistical technique. Ma thematical Biosciences 30:15-36, 1976.

13. Guess, H. A. and K. S. Crump. Maximum likelihood estimation of dose-response functions subject to absolutely monotonic cons $t$ ra ints. Annals of Sta tis tics 6:101-1 11, 1978 .

14. Guess, H. A., K. S. Crump, and R. Pete. Uncertainty estimates for low-dose rate extrapolations of animal carcinogenicity data. Cancer Research 37:3475-3483. 1977.
15. Hartley, H. O., and R. L. Sielken. Estimation of "safe doses" in carcinogenic experiments. Biometrics 33:1-30, 1977.

16. Heel, D. G., D. W. Gaylor, R. L. Kirschstein, U. Saffiotti, and M. A. Schneiderman. Estimation of risks of irreversible, delayed toxicity. J. of Toxicology and Environmental Health1:1 33-151, 1975.

17. Mantel, N. and W. R. Bryan. Safety testing of carcinogenic agents. J. Natl. Cancer Inst. 27:455-470, 1961.

18. Mantel, N., and M. Schneiderman. Estimating "safe" levels, a hazardous undertaking. Cancer Research 35:1379-1386, 1975.

19. Mantel, N., N. R. Bahidar, C. C. Brown, J. L. Ciminera, and J. W. Tukey. An improved Mantel-Bryan procedure for the "safety" testing of carcinogens. Cancer Research 35:865-872, 1975.

20. Mantel, N. Aspects of the Hartley-Sielken approach for setting "safe doses" of carcinogens. In origins of Human Cancer Book C. Human Risk Assessment, H. H.Hiatt, J. D. Watson, and J. A. Winsten (eds. ) (Cold Springs Harbor, N. Y.: Cold Springs Harbor Laboratory, 1977). pp. 1397-1401.

21. McCann, J., E. Choi, E. Yamasaki, and B. N. Ames. Detection of carcinogens as mutagens in the Salmonella/microsome test. Assay of 300 chemicals. Proc. Nat. Acad. Sci. USA 72:5135-5139, 1975.

22. McCann, J., and B. N. Ames. Detection of carcinogens as mutagens in the Salmonella/microsome test. Assay of 300 chemicals: Discussion. Proc. Nat. A cad. Sci. USA 73:950-954,1976.

23. Pete, R. Paper delivered at the NIEHS Wrightsville Beach conference on Low-Dose Risk Estimation, 1974.

24. Pete, R. The carcinogenic effects of chronic exposure to very low levels of toxic substances. Presented at an NIEHS conference on cancer risk estimation, Pinehurst, N. C., Mar. 10-12, 1976. Environmental Health Perspectives, 1977.

25. Pete, R. Epidemiology, multistage models, and short-term mutagenicity $y$ tests. In Origins of Human Cancer, Book C, Human Risk Assessment, H. H. Hiatt, J. D. Watson, and J. A. Winsten (eds. ) (Cold Springs Harbor, N. Y.: Cold Springs Harbor Laboratory, 1977 b), pp. 1397-1401.

26. Rai, K. and J. Van Ryzin. Risk assessment of toxic substances based on a generalized multihit dose response model. Proceedings of SIMS Conference, Alta, Utah, June 26-30, 1978.

27. Salsburg. D. S. Open query: Theoretical problems in the modified Mantel-Bryan procedure. Biometrics 33:4 19-421, 1977.

28. Watson, G. S. Age incidence curves for cancer. Proc. Nat. Acad. Sci. USA 74:1341-1342, 1977. 


\title{
Measuring Benefits and Costs*
}

\author{
by Donald S. Epp
}

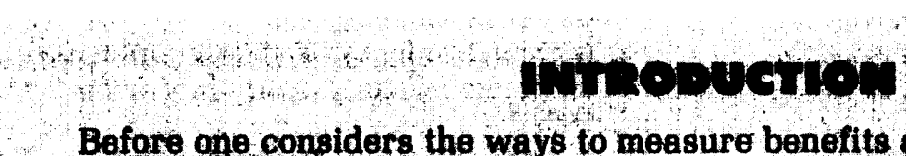

Before one considers the ways to meesure benefits and costs, it is essential to know preclesty what causes the benefits and costs. It would be possible to measure the benefits and costs of a particular tolerance level. Actually, what should be measured are the differences in effects between alternative tolerance levels, including no restriction at all. The findings of such an analysis would tell the decisionmaker whether the benefits of moving to the glven tolerance level exceeded the costs, but it would not tell whether that was the best tolerance level. It is possible that either higher or lower tolerance levels would increase the net benefits to society. The economic approach to setting tolerance levels requires consideration of several alternative levels and the changes in benefits and costs as one moves from one level to the next. The economic decision criterion is to lower the tolerance level so long as the additional benefits exceed the additional costs. When the costs of furthor restriction are greater than the benefits of such restriction, the efficient tolerance level has been found (assuming that bensfits and costs exhibit continuous relationships to tolerance levels that are at all points either concave or convex to the origin). Thus, the initial step in the enalysis is to choose a likely tolerance level and examine the consequences of moving from the status quo (no restriction) to the selected tolerance level

\section{Benefits}

The benefits to be evaluated are defined as the reduction in hazards to human health. This definition follows directly from the concern of the Food, Drug, and Cosmetic Act and the activities of FDA. Any effects on the health of animals or plants, except as they become food and thus affect the health of humans, is not considered in this report. This is not to say that economic analysis is incapable of considering other values associated with animal and plant life. Rather, it is to limit discussion to those aspects most germane to the decision under consideration.

The measurement of human health and the determination of a relationship between a particular contaminant in food and subsequent human health impairment must be made in biological, medical, and physical science terms. For example, the conclusion that daily consumption of food containing $x$ parts per million $(\mathrm{ppm})$ of a given

*Excerpt from OTA Wori ing Paper entitled "Priority Setting of Toxic Substances for Guiding Monitoring Programs. A complete copy of the paper can be obtained from the National Technical Information Service. (See app. J.) substance over a period of 3 years or longer will result in a 50-percent loss of function of the arms and hands is a medical and biological science conclusion. Until a determination of the health consequences is made, very little can be done toward comparing benefits and costs of regulation,

Health hazards from exposure to food contaminants may be stated in various ways depending on the health effects of the substance and on the state of knowledge about those effects. The hazard from a substance that increases mortality in the exposed population could be stated as the number of premature deaths per year. If illness requiring time-off from regular activity results from a certain exposure level, the hazard to the population might be stated as the number of person-days lost to illness per year. When it is not certain that exposure will cause a particular consequence, but that the likelihood is increased, probabilities may be attached to the outcomes. The hazard from exposure would then be stated that some percentage of the exposed population would die prematurely or would lose a specified number of days from normal activity due to illness, This method of stating hazard is encountered with cancer risks due to smoking tobacco. 
In many instances the substance in question may not have been studied for health effects or it may have been developed so recently that longterm effects of chronic exposure are uncertain. It may, therefore, be impossible to calculate probabilities, let alone specify the numbers of people affected, This does not mean the matter is of no concern. Based on the chemical structure of the substance, scientists may believe that the long-run consequences may be very serious. In such cases a precise probability cannot be attached to a specific event occurring, It cannot even be said with certainty that a health hazard exists. What can be said is that a serious health risk may be present and the consequences of that risk are so grave that society may wish to avoid whatever risk may result. While this type of statement of the health hazard is more difficult to use in a benefit-cost analysis, it is not impossible.

No matter how the hazard is stated, the first task in economic analysis is to convert the hazard statement into units of measurement that are comparable with the statements of costs to be developed later. Many (although not all) of the costs associated with those decisions are measured in monetary units-dollars. Likewise, at least some of the most important benefits to be achieved from restricting exposure to health risks are also measured in dollars. The most fruitful way to proceed, then, is to assemble the appropriate monetary measures and make conversion into monetary terms wherever possible for those effects not so measured.

\section{Cash Cost Approach}

One of the simplest ways to measure the cost of illness and at least part of the cost of premature death is to add up the expenditures made for treatment or burial and other out-of-pocket costs, These costs can be obtained in a straightforward manner and summed for the number of individuals that will be affected at the tolerance level being evaluated,

Even though the procedure does not involve complex calculation, this approach does involve decisions and judgments about the appropriate costs to us. Medical service costs vary greatly between procedures and between locations, It would be advisable, therefore, to obtain medical costs for the specific types of procedures re-

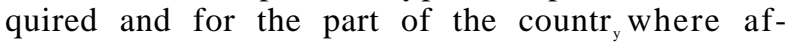
fected individuals would likely reside, In some cases where the contaminated food would likely be consumed nationwide, a weighted average cost of the appropriate medical service could suffice. . Where the contaminated food is consumed in particular regions of the country, e.g., catfish, the health effects would likely be concentrated in those regions also and the appropriate treatment costs should relate to the same regions.

While the cash cost approach is relatively simple to calculate, it ignores some of the true costs to society from an untimely death or an illness. One of the most obvious omissions is the failure to account for an individual's contribution to economic output, had sickness or early death not intervened. This weakness is corrected with the forgone-earnings approach.

\section{Forgone-Earnings Approach}

Generally, the forgone-earnings approach uses the discounted value of the future stream of earnings as the appropriate estimate for the cost of an untimely death, and thus, the appropriate estimate of the value of a life saved from a premature death. While it is clear that if an individual had not died or become ill in a particular year he would have continued to be productive for a number of years, it is not immediately obvious whether the correct measure to use is gross earnings or net earnings.

Those who support the use of gross earnings argue that what is of interest to society is lost production, and an individual's contribution to that production is measured by his or her earnings stream. Thus, the loss to society is the discounted present value of the stream of annual earnings weighted by the probability that the individual will be alive and well enough to earn that year's income. This approach is best suited to the analysis of illness rather than death and has been used to study the loss to society from mental illness, syphilis, and illness of all kinds collectively,

Those who prefer to use net earnings argue that, in the case of death, society may lose the production of the decedent, but it also no longer has to supply goods for the decedent's consumption. Death releases resources to the rest of society for their use. Thus, the appropriate measure of loss to society is the discounted present value to the stream of differences between an individual's expected income in each year and that individual's consumption during the same period. Each annual net earnings would be weighted by the probability of the individual surviving to that year. With this method of calculating, the cost to society of a death could be negative (or a benefit) if the individual consumes more than he or she produces. 
Retired and unemployed individuals are likely to have such negative life valuations.

The possibility of a negative value for a life has led to several criticisms of the approach. Some individuals produce products that are not marketed, but are none-the-less, real and valuable, The largest single example is the output of housewives. Other objections have been raised to the conclusion that the unemployed and retired are of no value. It must be admitted, however, that these latter objections appear to confuse the economic value of an individual with an ethical value of a human life, It is clear that the forgone-earnings approach examines only one portion of an individual's contribution to society-the contribution to those things measured in the net national product (NNP). If the goal of society is the maximization of NNP, then assessing the costs or benefits of a health-impacting project according to the forgone-earnings approach can be rationalized. If society holds other goals as well, then the use of forgone earnings is merely an expedient approximation.

\section{Willingness-to-Pay Approach}

The most telling argument against the previously discussed evaluation techniques is that they are conceptually incorrect for use in benefit-cost analysis. They are directed to find the answers to questions like "What is my life worth to other people like my heirs and society in general?" The conceptually correct question to ask is "How much would I pay to avoid a small probability of my death or illness?" Approaches that answer this question are consistent with other measures of societal welfare since they estimate the aggregate consumer surplus involved in the reduction in mortality or morbidity rates.

What has come to be known as the "willingness-t o-pa y" approach has received extensive treatment in recent economic literature. A review of much of the theoretical literature and the discussion of some technical points related to a person's willingness to pay for small changes in mortality rate may be found in Epp, et al. (ch. 4), That review will not be repeated here, but rather, the remainder of this section will be addressed to reviewing techniques for determining an individual's willingness to pay. Two approaches have theoretical validity: compensation for risk-taking and questionnaires of willingness to pay.

Compensation for risk-taking as a technique for estimating the loss in consumer surplus due to increased risk of death, illness, or injury, has the advantage of being observable to the marketplace. If an individual agrees to undertake a hazardous occupation which increases his probability of death in exchange for a given sum of money, that sum can be used as an estimate of his willingness to pay for safety, Thaler and Rosen used data that measures the relative riskiness of jobs to estimate the tradeoff between wages and risk. Thirty-seven broadly defined job classifications shown to be actuarially riskier than the average occupation were matched against a cross-sectional earnings survey. The results showed that individuals in the risky occupations received increased compensation of about $\$ 200$ per year (1967 dollars) for jobs where the risk of death was 1 in 1,000 greater than the average.

This estimate may be conservative when applied to the general population. The occupations surveyed in the Thaler and Rosen study are approximately five times riskier than the average U.S. occupation. People who take these jobs have different reservation prices for risk than individuals who pick less risky jobs. The derived estimate of compensation required to offset greater risk is, therefore, an extremely conservative approximation of aggregate willingness to be compensated. A further rationale for believing this estimate to be conservative is that the riskiness of these jobs can in some measure be affected by the individual employee. $\mathrm{He}$ or she is to some degree in control of the personal risk level within the context of the risk level of the occupation. If the individual is not able to affect his or her personal risk exposure, it is quite likely that they would need to be compensated to a greater extent to undertake the unwanted risk.

The other technique for assessing willingness to pay is the questionnaire method. A representative sample of individuals are asked how much they would be willing to pay to achieve a specified change in a particular condition. For the problem of environmental contamination of food, the question would most likely be directed toward the willingness to pay for a reduction in the probability of suffering specific health effects, such as speech impairment and nerve sensations from exposure to methylmercury. Questionnaire design ranges from direct questions about dollar amounts for a specific risk change to a series of questions about items related to the specific risk change of interest from which the analyst can infer an answer to the "what would you pay?" question,

While the willingness-to-pa $a_{y}$ approach is asking the conceptually correct question, it has seri- 
ous difficulties in application, because the items of interest are going to be consumed in equal amounts by everyone and no one can be excluded from consuming the item if it is provided for anyone. For example, a reduction in the risk of lung disorders due to reduced sulfur dioxide levels in the air will be available to everyone in the area regardless of their preferences or payment, If it is provided for one person, it is impossible to exclude others. The communal consumption aspect of this large class of similar goods and services provides an incentive for people to misrepresent their true preferences when asked, For instance, if a person believes he will be required to pay some amount in proportion to his answer yet there is a high probability the good will be provided whether or not he contributes and if it is provided, he will be able to enjoy it, then it is rational for him to respond that he is unwilling to pay anything for it, even if he knows he really would pay some amount, if necessary. On the other hand, if an individual knows that someone else will pay for the good if it is found to be valued highly will find it rational to grossly overstate its value to him. Thus, the incentive to be a free rider makes it difficult to accurately measure the value to society of an improvement in food safety.
In spite of the difficulties associated with empirical measurement of willingness to pay, several studies have been done and have reported some success with various methods of presenting the question to the respondents. Randall, et al. (1974) used pictures of the powerplant at Fruitland, N. Mex., at various levels of smoke emission to elicit responses from residents and tourists as to the amount they would pay (in the form of a sales tax increase) to move from a less preferred view to the respondent's preferred view. The technique was used in a similar fashion to examine questions about a powerplant location in southern Utah (Brookshire, et al. ) and about reclamation of stripmined land in Kentucky (Randall, et al., 1978). A modification of the technique was used by Mann, et al. to determine willingness to pay for small changes in human mortality. Their study found that people are able to comprehend risk changes of the order of $10^{-6}$. Although the risk premiums stated in the Mann, et al. study were substantially different from those' found by Thaler and Rosen, the study indicated that individuals were able to answer the type of question posed, and that with improvements in the survey instrument, the willingness-to-pay questionnaire may be a workable approach.

\section{COSTS}

The costs associated with establishing a particular tolerance level are the loss of social welfare resulting from the reduction in supply because some otherwise useful food products can no longer be sold for consumption. The reductions in consumer and producer surplus, which are discussed in detail in this section, account for the economic value of resources, such as labor and production capital, which may no longer be employed in producing the food product being considered, It is possible, however, that some resources may not have any alternative employment, In such case the tolerance level may cause these resources to be unemployed. While this lack of alternative employment is not strictly an economic cost, it is clearly a consequence that a decisionmaker may wish to consider. Therefore, other effects (some might wish to call them costs) of the decision are examined in the following section entitled "Distributional Effects."

The starting point for assessing the costs of moving to a particular tolerance level is the physical product that will be affected. The results of the monitoring program and various special test procedures can tell what proportion of various food products from any specified area will not meet the tolerance level. The task of the economic analysis is to evaluate the impact of such a reduction in the supply of that food product.

When analyzing the effects of a tolerance level that removes some food products from the market, there are two major alternative methods. These two are what have been called the alternative cost method and the opportunity cost method. Either of these methods may employ models of the affected industry, but as will be shown in the following sections, the opportunity cost method requires more sophisticated models and delivers a more comprehensive description of likely effects.

\section{Alternative Cost Approach}

With the alternative cost method, the analyst examines the additional cost necessary to achieve a given objective using the next best alternative method to the one under study. Applying this to the PCB contamination of lake trout in Lake Superior, for example, would require that the analyst 
examine the next best method for producing $164,000 \mathrm{lbs}$ of lake trout that did not exceed 2 ppm of PCB to replace that amount from Lake Superior which does exceed $2 \mathrm{ppm}$ of PCB. This approach requires that there be some alternative method available which will achieve the same level of output in order that the costs of the two alternative ways of producing the output can be compared.

If there are several alternative methods available for producing a "replacement" amount of production, the alternative cost approach requires not only that the cost of total replacement with each method be examined, but that combinations of methods be considered also. To properly evaluate the alternative cost figure, the analyst must know the costs of various amounts of product from each alternative method. To continue with the PCB contamination of lake trout example, the analyst must consider not only the cost of 164,000 additional lbs of lake trout with less than 2 ppm of PCB from each of the other Great Lakes (and any other large sources), but the costs of various increments of production, such as 10,000 lbs. from each source. The least cost combination of increments would be found by starting with the least costly way of obtaining 10,000 additional lbs and adding to that the next least costly way of getting 10,000 more lbs until a total of $164,000 \mathrm{lbs}$ are accounted for.

\section{Opportunity Cost Approach}

With the opportunity cost method the analyst examines the additional cost necessary to achieve a new market equilibrium amount of a food product which reflects the likely higher price and, therefore, lower consumption of consumers. Since restricting the supply of a food product from previous sources means a shift to higher cost alternative sources, we can use our knowledge of markets and people's preferences for the food product to estimate their adjustment to the restriction, This gives a different estimate of costs due to restriction than does the alternative cost method which assumes no change in the amount of the food product consumed.

A simplified partial analysis of the difference between the alternative cost method and the opportunity cost method is presented with the use of figure E-1. The decision to establish a tolerance level such that some portion of the product currently offered for sale may no longer be sold is shown by the shift in the supply curve. Curve $S_{\text {" }}$ represents the industry supply curve prior to the change in tolerance level, while curve $S_{1}$ represents supply under the higher cost next best alternative method of production which does not violate the new tolerance level. The alternative cost method examines the increase in cost of obtaining the initial equilibrium quantity, $Q_{0}$. This increase in cost is represented by the area $\mathrm{ABP}_{1} \mathrm{P}_{0}$. The opportunity cost method, on the other hand, recognizes that a new equilibrium price and quantity will emerge after the shift in supply. Quantity will shift to $\mathrm{Q}_{1}$ and price to $\mathrm{P}_{2}$. Comparing the new equilibrium with the old, the analyst using the opportunity cost method observes that the consumer surplus has been reduced by an amount equal to area $\mathrm{ACP}_{2} \mathrm{P}_{0}$ in figure $\mathrm{E}-1$. This reduction in wellbeing is clearly less than the one calculated with the alternative cost method. Because the opportunity cost method recognizes changes in production and consumption, it is the preferred method. The alternative cost method tends to overstate the cost of more restrictive tolerance levels.

Figure E-1 .-Analysis of a Shift in Supply

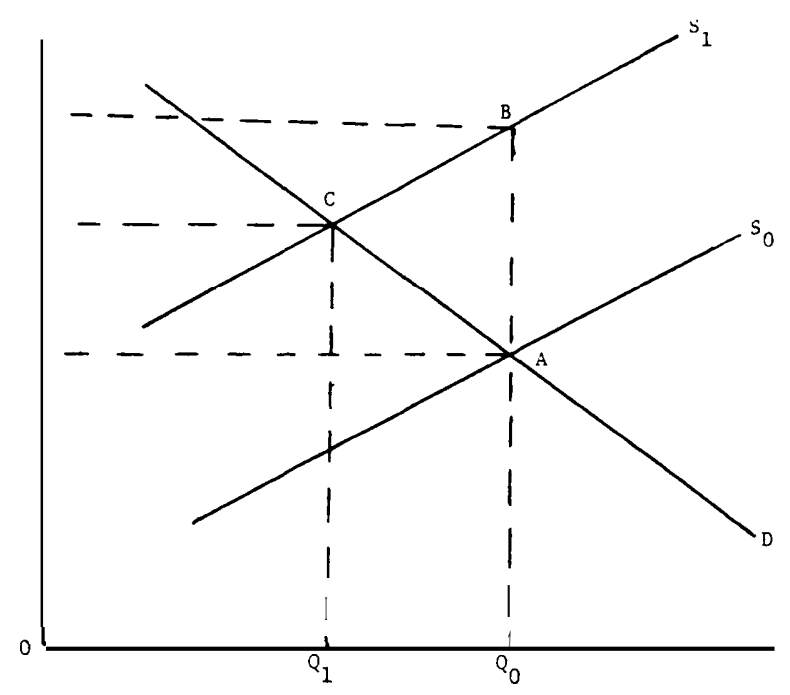

The above diagrammatic analysis presents the essential features of the budgeting approach to the opportunity cost method. This is discussed in more detail below. Another approach, modeling, carries the above analysis further to show changes in the market for factors of production as well as other product markets, While this method cannot employ graphic analysis due to the complexity of relationships, the mathematical modeling techniques described below permit a much more comprehensive analysis of likely effects of a change in tolerance level. 
As was noted above, the opportunity cost approach measures effects of a change in tolerance level as the change in consumer surplus in the economy. There is abundant literature discussing the problems of estimating consumer surplus; some authors even question the value of the concept in many empirical contexts. For this exercise, however, it seems appropriate that consumer surplus be used. The ultimate consumer of the contaminated food products should provide the basis for evaluation. This becomes particularly crucial where ramifications of the production shift may include a variety of products, not just the contaminated product. Consumer surplus becomes the common denominator allowing comparison among a variety of production effects. In practice, the opportunity cost approach may employ either of two methods: budgeting or modeling.

\section{Budgeting}

With budgeting the analyst acknowledges that production shifts are likely for the contaminated product. This technique uses data on inputs used in the production of a product to calculate the costs of producing a particular amount of that product, assuming that other commodities will be produced in the same amounts and at the same prices as with the status quo. The budgeting approach is a more limited and restricted approach than the modeling one, but for some circumstances may prove advantageous.

\section{Modeling}

The second and more complete method of applying the opportunity cost approach is through the use of production models. With this method, mathematical models of the relevant portion of the economy are employed to trace the shifts in supply curves and the changes in the amount and price of various commodities and factors which result from the restriction on the use of a contaminated product. If these models are specified to include geographic areas and the various alternative production activities which take place in each of these areas, they are able to project changes in the location of production of particular crops and changes in the use of various production factors in each region of the country. This more realistically describes the likely reactions to the change in tolerance level and permits the calculation of a more accurate estimate of the change in consumer welfare resulting from the action level decision.

\section{Comparison of Approaches}

The alternative cost and opportunity cost approaches are not equally adapted to handling all problems-each has several advantages and disadvantages. The alternative cost approach has the advantage of using data that is more easily obtained and not requiring extensive development of mathematical models prior to the analysis. Since the alternative cost approach compares the status quo with the next best alternative, subject to the change in tolerance level, the only additional data needed beyond present production techniques is an estimate of how the same amount of product could be produced under the best alternative available with the new tolerance level. This data can frequently be obtained from experts in the production of the food under consideration.

The major disadvantage of the alternative cost approach is that it is conceptually erroneous. Our knowledge of economic adjustments to changing production conditions recognizes that adjustments will be made in the enterprise combinations and in factor combinations for producing a particular food product. If a significant portion of the total production is removed from use or a specific location is no longer capable of producing a safe food product, the ensuing production adjustments will not be limited to factors of production, but may also include changing the foods produced or the location of production. The amount consumed of the food for which a new tolerance level is being established will probably adjust to changes in the cost of obtaining the food in a safe form (within tolerance levels). The alternative cost approach ignores all of this knowledge by assuming that the food in question, using the next best alternative, will be produced at the same level as before.

The conceptual error leads to a second errorthe overstatement of the costs associated with the tolerance level, Because this approach ignores the adjustments in the amount of a food product produced, it leads the analyst to a cost figure that is greater than the one that would actually result. As production costs increase it is likely that the quantity produced will decrease. Thus, the alternative cost approach has the analyst multiply a higher cost per unit by more units than would actually be produced. This leads to an erroneous calculation of the change in consumer surplus and to the overstatement of the cost of setting tolerance at a particular level. 
The major advantage of the opportunity cost approach is conceptual: it allows the analyst to see the adjustments that the economy is likely to make in response to changing production costs as a new tolerance level removes some of the product from the market. Where agricultural products are involved, the use of an econometric model of the agricultural sector facilitates the analysis of changing comparative advantage and the resulting production pattern, This gives a more accurate indication of the ultimate cost of making the tolerance level decision,

The use of models can also facilitate distributional analysis if those models have spatial (regional) variables introduced into them. Through the use of regional supply and demand models, the analyst is able to estimate not only the market equilibrium supply and demand adjustments, but also the regional production adjustments in response to the overall market changes. It is thus possible to note changes in the regional location of production and to estimate the impact of these changes on various income and social groups that are distributed differently in the various regions of the country. Thus, the models that are used for the opportunity cost approach can facilitate a more detailed and sophisticated analysis of the effects of a change in the tolerance level. The reasons for examining distributional effects as well as a brief description of some ways these may be done are included in the next section of this paper.

The major disadvantage of the opportunity cost approach is that it requires a very large amount of data. Production costs, outputs, factor requirements, factor prices, and a variety of other bits of information are required for each alternative food production activity in each region including alternative ways of producing the food under consideration. Even if the supply equations and activities are limited to those most likely to enter into the solution, the requirements are formidable for most agricultural and fishery commodities. The demand side also requires regional consideration with specification of the demand for each of the commodities included in the supply side of the model. Careful attention must be given to the inclusion of complements and substitutes so that the model will give a reasonable approximation of actual market adjustments.

A second disadvantage of the opportunity cost approach is that the models developed for analyzing production and market shifts are usually short-run and static, This means that these models must be revised periodically in order to in- clude new developments in factor prices, product prices, and production technology. Thus, the models are expensive to maintain. They are also expensive to create in the first place. Most models require a great deal of prior research on the technical relationships in production and specification of the factors related to consumption. While much of this work has been done for agricultural commodities, it is recognized that the material is frequently inexact and often the models are out of date, It would be necessary, therefore, to undertake some rather expensive research in order to incorporate the opportunity cost approach for a commodity that did not already have substantial prior work. This is likely to be the case for seafoods, freshwater fish, and certain agricultural products produced in a few local areas.

Obviously, the detailed knowledge of production relationships and therefore the expense is reduced if one uses the budgeting method rather than the modeling method in the opportunity cost approach, Budgets usually involve a partial analysis of the adjustments and therefore do not require the development of production relationships for commodities not closely related to the commodity under consideration, Even so, the budgeting approach requires more information than the alternative cost approach because of the consideration of production changes. It is unlikely that the budgeting approach can be used for a regionalization analysis that involves more than a very few regions. Thus, the lesser cost produces less information.

Since the two approaches, alternative cost and opportunity cost, differ in the amount of data that they require and in the cost of acquiring and processing the data, it is necessary for an analyst to choose between these two methods. Several points should be considered when making a selection of the most appropriate analytical method.

\section{Demand Elasticity}

If the product for which the tolerance level decision is being made has a very inelastic demand, ' it may be appropriate to use the alternative cost approach. With a very inelastic demand, it is likely that the assumption of producing the same quantity regardless of cost is a reasonably close approximation to reality, Thus, the disadvantages enumerated above for the alternative cost approach are of less significance for many agricul-

Inelastic demand means that the percentage change in quantily de: mand as the price of a gund ehanges is smaller than the percentage change in price. Perfectly inelastic demand describes the caste where there is no change in the quantily demanded when the price rhanges. 
tural products, such as certain vegetables, sugar, and cereals for human consumption, as well as for fish.

\section{Information Availability}

Since the opportunity cost approach requires a great deal of information about production relationships, not only for the commodity under consideration but for alternative commodities, it is helpful to have these relationships previously developed. Much of this information is so time consuming to develop that it would be virtually impossible to create a research program that could give results in time to be useful for any action level decision if a great deal of groundwork had not been laid prior to that research effort. If no information is available concerning production of the product in question or the alternative product that might be produced or consumed, it is likely that the alternative cost approach would be adopted. This approach requires less data and the material that it requires probably can be produced in time to be useful in making a tolerance level decision.

\section{Complexity of Interrelationships}

If a product is produced in many areas with a large variety of production alternatives, both with regard to factors of production and to alternative products, one needs to use an opportunity cost approach. In such a situation the interrelationships are so complex that it is difficult to judge from observation of the data the likely combination that will result from a tolerance level decision. Under these conditions it is very desirable to use the opportunity cost approach if at all possible.

\section{Availability y of Mathematical Models}

The stage of development of the mathematical programing models for a particular product or sector of the economy is an important consideration in choosing a method. If programing models are fairly well-developed for most of the important alternative commodities as well as the commodity under consideration, it may be possible to modify the existing work relatively inexpensively to obtain the information needed for the tolerance level decision, For example, a great deal of work has been done with the feed grain-food grain sectors of American agriculture. Less work has been done on the livestock sectors, although there are some models that incorporate feed grains and livestock and some models include the dairy subsector. If the decision to be made involves food or food grains, serious consideration should be given to using the opportunity cost approach and some of the models that have been developed, with whatever modification seems appropriate. On the other hand, there has been very little modeling that includes specialty crops, such as specific fruits and vegetables, into a general agricultural model. It would be expensive and of dubious value to develop a programing model for a tolerance level decision involving those crops.

It is readily apparent that the key assumption of the alternative cost approach-no change in the quantity of product produced after restricting the amount of a particular contaminant that may be present-is not realistic in most cases and can lead to substantial error in estimating the social costs of a decision. It is the opinion of the author that the alternative cost approach is appropriate only in those cases where an alternative source of the product is available at virtually the same effective price or where alternative production techniques are available at no increase in per unit cost of production and which would involve not more than negligible shifts in the location of production. These are very restrictive conditions. For most tolerance level decisions involving major agricultural products these conditions do not hold and the opportunity cost approach is strongly advised.

\section{DISTRIBUTION EFFECTS}

The previous two sections have outlined considerations and methods for determining the benefits and costs of a change in tolerance level for a particular contaminant in a specific food product. From an economic perspective and a society-as-awhole viewpoint, these sections cover the economic analysis of welfare changes. There are, however, related areas of concern which may be crucial to the social acceptability of a decision and for which economic analysis can be useful. Of ma- jor interest here is the analysis of how a decision impacts on various groups within society. Even though a decision may produce an improvement in welfare for the whole of society, there may be particular groups for which welfare is reduced. Such distribution effects may be due to the benefits and costs being shared differently by different income groups or by different regions of the country, There may also be concern about how the consequences of the decision affect the 
environment in various locations or its effect of esthetic considerations. Each of these points is reviewed briefly in this section.

The analysis of benefits and costs may show greater addition to benefits from a particular tolerance level change than the addition to costs; thus, a desirable decision, The consequences of that decision may not, however, be desirable for all affected parties, It is, therefore, important in some cases to examine the distribution of benefits and costs among social and economic groups.

For example, the decision to set a very restrictive tolerance level on a particular contaminant in fish may mean that fishing in certain bodies of water is no longer possible. That may have a very slight effect on consumers, since they can easily switch to fish from other areas. The fishermen who lose their employment may, however, have much lower incomes than the average consumer and may have few or no alternative sources of employment. In this case the benefits in health hazard avoided by a large number of people may greatly exceed the costs of idled resources in fishing and thus, the decision is appropriate. It is well for a decisionmaker to realize, however, that the major portion of the costs will be borne by people of much below average incomes with few alternatives to mitigate the losses.

Another decision may involve restriction of contaminant levels in a food eaten primarily by a specific ethnic or racial minority whose members have low incomes, The producers of this food may have several alternative products they can produce and the consumers may also find substitutes readily available. In this case the costs are slight and a low-income minority receives substantial health benefits.

Most of the data needed to describe the distribution of effects among income groups is available from the analysis of benefits and costs. In some cases it is not clear which income or social group is affected, but the analyst can usually determine this at the time he collects the data on effects. Thus, it is important that benefit-cost analysts be alert for information about the incidence of effects as they collect their data. What may be relatively easy to determine while the original source is being contacted may be time-consuming and difficult to find out at a later point in the analysis. The presentation of distributive impacts would probably be in narrative form rather than a quantitative analysis. The important point is to include this additional information for the decisionmaker so that the fullest possible knowledge of impacts is available when the decision is made.
The interregional differences in the impact of benefits and costs may also be of interest to the decisionmaker. Frequently the alternative source of product to replace that which is contaminated above the new tolerance level will be in a different area. Where a particular stream or valley produces contaminated food, the production may shift only a few miles to a nearby river or valley. Then the impact on the economy of a particular community may be slight. In other cases, a widespread area, such as a large estuary or part of a State, may be contaminated and production will shift to a completely different part of the country, Interregional differences may arise from products which are produced in one area, but consumed in another, For example, most of the winter head lettuce eaten in the United States is grown in the Imperial Valley of California, yet only a minuscule portion of the crop is consumed there. If a tolerance level for some contaminant made it impossible to sell Imperial Valley lettuce, almost all of the cost would be borne by that small part of California while the health hazard reduction would be shared throughout the rest of the country.

The data for constructing the regional impacts of a tolerance level decision can be found most easily in mathematical programing analyses under the opportunity cost approach. Not all programing models have regional specifications, but most agricultural models do, Careful review of budgeting results by experts on the production and marketing of the foods in question can also reveal some of the regional impacts or can call attention to the likely importance of regional differences. Further regional analysis can be performed if desired. The alternative cost approach does not provide much data on regional impacts. Here again, however, a careful review of the results by a knowledgeable analyst can suggest the likelihood of regional shifts that warrant further analysis with finer detail on regional specification.

The fact that a particular decision will severely harm a particular region or social group does not necessarily imply that the decision should be avoided. If the society-wide analysis of benefits and costs suggests a net gain from the decision, there is good reason to go ahead. The analysis of the distribution of effects may serve to point out the need for special programs to assist those harmed adjust to the decision. For example, regional impact analysis may point out the need for a retraining program for unemployed workers in a particular region far enough in advance that the 
program can be ready to go when the tolerance level decision takes effect.

The analysis of impacts on various regions and social groups can provide the starting point for analysis of the sociological and psychological impacts of the tolerance level decision. In some, perhaps many, cases the effect on a particular community or group of people may have serious consequences for social structure, organizational stability, and even the mental health of the affected people. Such a possibility was pointed out and briefly discussed by O'Mara and Reynolds (PP. 61-66) in their analysis of restricting fishing in the James River of Virginia due to kepone contamination. Since the present report is directed toward economic analysis, the techniques of sociological analysis will not be discussed here. Some of the analyses likely to be used are briefly outlined in Epp, et al. (pp. 134-138).

Another area of potential concern is the impact of a tolerance level decision on the environment or on the esthetic properties of a region. It is possible that a tolerance level decision may cause production to shift to an area where environmental damage would increase.

For example, prohibiting the sale of milk from one area may cause production to concentrate in another, leading to increased wastes from dairy farm holding pens and milk-processing plants. What may have been an acceptable load on the environment previously may become excessive. Analysts should be alert to the possible environmental and esthetic impacts of decisions. In some cases these effects can be measured in monetary terms and compared with benefits and costs. In other cases, the effects must remain in biological terms, but described in such a way as to make them useful in a tolerance level decision. The use of environmental food chain models and impact matrices has been suggested by the author with respect to pesticide decisions (Epp, et al., chs. 5 and 6) and the interested reader is referred to that discussion for greater detail, Not all tolerance level decisions will involve a significant environmental effect, but the procedure of analysis should include a notation to check for such effects and an analytical protocol for use when needed.

\section{ALTERNATIVE TOLERANCE LEVELS}

The sections on benefits, costs, and distribution effects have described how an analysis might be conducted of a particular decision to change the tolerance level from one point to another. For example, one decision might move from no restriction to prohibiting the sale of a particular food with $5 \mathrm{ppm}$ or more of a specific contaminant. If benefits exceed costs and distributional as well as other effects are not overwhelming, then the move to a 5-ppm tolerance is efficient. That does not say that $5 \mathrm{ppm}$ is the most efficient tolerance level. There may be some other level, say 2 ppm, that would produce even greater net benefits. The proper use of economic methods requires that consideration be given to the likely changes in benefits and costs if the tolerance level were changed a little either way. If it seems possible that net benefits would increase significantly by moving to a different tolerance level, then a detailed analysis as described above should be made of the change from the originally proposed tolerance level (5 ppm in the example) to the new level (2 ppm). 


\section{LITERATURE CITED IN APPENDIX E}

Brookshire, D. S., B. C. Ives, and W. D. Schulze. 1976, "The Valuation of Aesthetic Preferences," Journal of Environment tal Economics and Management $3: 325-346$.

Epp, D. J., et al., 1977, Identification and Specification of Inputs for Benefit-Cost Modeling of Pesticide Use, EPA-600/5-77-01 2, U. S. Environmental Protection Agency. Washington, D.C.

George, P. S., and G. A. King, 1971, "Consumer Demand for Food Commodities in the United States with Projections for 1980," Giannini Foundation Monograph Number 26, California Agricultural Experiment Station, Berkeley.

Lewis, P. F., 1977. "Economic Impact Assessment for Proposed Reduction of Temporary Tolerances for Polychlorinated Biphenyls in Food. "” Unpublished manuscript, U.S. Food and Drug Administration, Washington, D.C.

Mann. S. H., et al., 1976, "The Determination of the Economic Effects of Pesticide Use on Human Health: A Gaming Approach to Willingness-to-Pay, "Working Paper Series No. 32. The Center for the Study of Environmental Policy. The Pennsylvania State University, University Park, Pa.

McNally, J. M.. 1978a, "Polycholorinated Biphenyls: Environmental Contamination of Food," Congressional Research Service, Washington, D. C., OTA Working Paper.
McNally, J. M., 1978b, "Polybrominated Biphenyls: Environmental Contamina t ion of Food, " Congressional Research Service, Washington, D. C., OTA Working Paper.

O`Mara, G. K., and R. R. Reynolds, 1976, Evaluation of Economic and Social Consequences of Restricting Fishing Due to Kepone Pollution in the James River, Virginia. U. S. Environmental Protection Agency, Washington, D.C.

Randall, A. B., et al.,1974, "Benefits of Abating Aesthetic Environmental Damage from the Four Corners Power Plant." New Mexico State University Agricultural Experiment Station Bulletin, Las Cruces, N. Mex.

Randall, A., et al.. 1978, "Reclaiming Coal Surface Mines in Central Appalachia: A Case Study of Benefits and Costs," Land Economics 54:472-489.

Reichelderfer, K. H., et al., 1979, “'Pesticide Policy Evaluation Models," NRED Working Paper (unnumbered draft), Natural Resource Economics Division, Economics, Statistics, and Cooperatives Service, U.S. Department of Agriculture, Washington,D.C.

Thaler. R., and S, Rosen, 1973, The Value of Saving a Life: Evidence from the Labor Market. National Burea $u$ of Economic Resea rch, Con ference on Income and Weal $\mathrm{t} h$.

'1'racer Jitco, Inc., 1978, "Risk Assessment of Polybromina tedBiphenyls, "O'TA Working Paper, 


\section{Priority Setting of Toxic Substances for Guiding Monitoring Programs*}

by Clement Associates, Inc.

\section{INTRODUCTORY REMARKS ON THE ESTABLISHMENT OF PRIORITIES}

There are many purposes for which the Federal Government must set priorities among toxic chemicals in the environment. The universe of environmental contaminants is very large, and resources for research, testing, monitoring, and regulation are limited. Some statutes explicitly require Government agencies to set priorities for testing or regulation, and in many other cases agencies may be called on to explain their decisions to tackle some problems before others. Accordingly it is very desirable to develop rational systems for setting priorities.

Establishment of priorities involves preparing a list of problems, ordered according to their perceived *'importance" or urgency, before attempting to solve them. It is by definition a preliminary step and should be designed to maximize the efficiency of the problem-solving process, Although it is desirable to use as much relevant information as possible in setting priorities, it is also possible to use too much information. If too much time is spent in overelaborate priority-setting exercises the gain in efficiency achieved by tackling the most urgent problems first is offset by a delay in taking any action at all. The first task in designing an efficient priority-setting system is thus to make a reasonable compromise between the effort expended on this preliminary step and the gain in efficiency achieved by doing it well.

Priority-setting exercises are usually limited primarily by the unavailability of data. (If extensive data are available, then it is usually obvious which problems should be tackled first. ) For this reason there is usually little to be gained by developing elaborate multifactorial schemes to rank

*Excerpt from OTA Working Paper entitled "Priority Setting of Toxic Substances for Guiding Monitoring Programs." A complete copy of the paper can be obtained from the National Technical Information Service. (See app. J.) problems in numerical order. Good systems for establishing priorities will inevitably involve a substantial element of sound scientific judgment in weighing and interpreting incomplete and unsystematic data. The second task in designing an efficient priority-setting system is thus to make a reasonable compromise between the use of objective criteria and scientific judgment.

This report is concerned specifically with the establishment of priorities for monitoring toxic chemicals in food. There are two general methods by which this might be done: 1) directly, by sampling the food supply for chemical contaminants and ranking them according to potential hazard; and 2) indirectly, by surveying the universe of industrial chemicals and ranking them according to their potential for entering the food supply in toxic amounts. The first method has obvious advantages and in fact has been pursued by FDA. The method is, however, limited by the available analytical methodology: specifically, most of the chemicals recognized to date as food contaminants are those relatively easily detected by gas chromatography or atomic absorption spectrometry. The principal question addressed in this report, therefore, is whether the second method can complement the first by identifying potentially significant contaminants that have not yet been detected in foods. Although this report indicates that it can do so, it should be recognized that the second method also is limited to some extent by available analytical methodology. The factors we can use to identify the potential of a chemical for entering the food supply are selected on the basis of a knowledge of the properties and environmental behavior of other chemicals already known to do so. This in turn is based on our knowledge of the extent of contamination and hence on our analytical capabilities. Thus, there is an inherent bias towards identifying as potential food con- 
taminants those chemicals that are similar to chemicals already identified in food. This bias can only be offset by the use of good scientific judgment. This point illustrates a general problem in all systems for setting priorities: chemicals on which there is no information at all will automatically be given low priority, unless some room is left for largely intuitive judgments,

\section{CRITERIA AND METHODS USED IN PREVIOUS EFFORTS TO ESTABLISH PRIORITIES AMONG TOXIC CHEMICALS IN THE ENVIRONMENT}

\section{Survey of Toxic Chemical Priority Lists}

Thirty-two priority lists of toxic chemicals compiled during the last 5 years have been identified. Most of these lists (28 out of 32) were prepared for the Federal Government. For each list, a description sheet indicating the purpose of the list and the criteria used in selecting chemicals was prepared. In addition, the name of the list, the Federal agency or other institution for which the list was compiled, the date of completion, the contract or document number, type of substances, the number of chemical substances, the means by which the chemical was identified (for example, CAS number or chemical name), whether the list is machine readable, and relevant comments were included.

\section{Methods}

\section{Chemical Selection}

Compiling a comprehensive list. The first step in priority-setting efforts is usually compilation of a comprehensive list defining the universe of chemicals under consideration. Since chemical compounds and mixtures are often referred to by several different names, each chemical compound on the comprehensive list should be uniquely identified. This can be done by specifying for each chemical on the list the IUPAC chemical name, synonyms, molecular structure, and/or the Chemical Abstracts Service (CAS) registry number. The use of CAS numbers facilitates computerization.

Most often, the source of chemicals for this comprehensive list is previously existing lists. Errors of omission are likely with this method. A chemical not considered previously will not be considered by someone using existing lists as a source, The more comprehensive the source lists, the less likely chemicals are to be omitted from consideration. An alternative method for selecting chemicals for consideration is reliance on recommendations of experts. The likelihood of errors of omission when this method is used will depend on the extent of knowledge and experience of the experts. These methods can be combined by supplementing source lists with chemicals of concern to experts.

Reducing the size of the comprehensive list. It is almost always necessary to reduce the size of the comprehensive list before ranking. Since the resources needed for compiling and evaluating the necessary data for setting priorities are usually limited, a "narrowing" or "truncating" step is performed. Errors are very likely to occur at this step because, for practical reasons, decisions at this stage are usually based on incomplete information, In a multistage priority selection scheme, such as the one by which the TSCA Interagency Testing Committee selected chemicals to recommend to the EPA Administrator for testing, additional information is obtained at each stage of reduction. In that effort, an initial listing of 3,650 substances was reduced to a master file of 1,700 substances, then to a preliminary list of 330 substances from which approximately 100 chemicals were selected for dossier preparation. Twenty-one chemicals or groups of chemicals from that list have been designated for recommendation by the committee. In this type of process, the earliest stages may be the most subjective and the most likely to result in errors of omission.

Grouping. Individual chemicals are often combined in groups or classes. This is usually done in an attempt to reduce a list of chemicals to a more manageable set, There are, however, other reasons for grouping. Some monitoring or analytical techniques do not distinguish between closely related chemicals. Some classes of chemicals, such as fluorocarbons, may be considered as a group for regulatory purposes. Such chemicals as PCBs are not manufactured or used as individual chemical compounds and are, therefore, nearly always considered as a group. Groups can be based on chemical and physical properties, on chemical 
structure, on uses (e.g., flame retardants, fluorescent brighteners), or on some combination of these factors. Sometimes a single compound or several compounds are selected as representative of the group.

An advantage of grouping chemicals with similar structure or functional groups is that it sometimes allows chemical, physical, and biological properties of individual compounds to be predicted. Members of a chemical class may behave in a qualitatively similar fashion. In addition, some properties increase or decrease systematically along homologous series. Attempts to derive structure-activity correlations are much more likely to be successful for chemical and physical properties than for biological properties. Because of some unique properties of biological systems, such as enzyme specificity, it is possible for closely related chemical compounds, even such stereoisomers as aldrin, dieldrin, and endrin, to have very different biological properties.

Some weaknesses associated with grouping are:

- Members of the group may vary widely with respect to the factor being scored, making it difficult or meaningless to assign a single score to the group.

- No single compound can truly represent an entire class.

- It is often difficult to assign compounds with several different functional groups to a single chemical class. This difficulty can sometimes be overcome by computerized substructure search systems that allow all chemical compounds with a specific functional group to be retrieved regardless of the presence or absence of other functional groups.

At one stage of the National Science Foundation (NSF) Workshop Panel, multistage effort grouping was done in an attempt to reduce the list of chemicals to a more manageable set. Fourteen chemical classes were defined, and a representative chemical was identified for each. Weaknesses the panel found to be associated with this procedure included:

- The classes were not completely valid either chemically or functionally.

- The physical and biological properties of compounds in a given class varied widely.

- No single compound was truly representative of a class.

The class concept was abandoned in the next stage of the effort and selected members were considered as individual chemicals.

\section{Determining Factors To Be Considered in Setting Priorities}

The purpose for which the list is to be used must be clarified. Once the problem the priority list is intended to solve is understood, factors relevant to that problem can be determined. Criteria based on those factors can then be formulated for assigning chemicals a place on the list. General criteria must often be defined in terms of specific parameters for which information is available. Those parameters for which quantitative data are readily available are the easiest to use for scoring and ranking. On the other hand, data are frequently lacking on the factors of greatest relevance to the specific problem. In such cases factors of less relevance may have to be selected. The choice of factors to be used in setting priorities often involves a compromise between the factors that are most relevant and the factors on which sufficient information is available.

\section{Assembling Data on Each Chemical}

Sound decisions on assignment of priorities require reliable data on each chemical to be ranked. The available information may, however, be massive, contradictory, unverifiable, scanty, or absent. It is usually difficult or impossible to obtain existing information that has been classified as proprietary or confidential.

When information is needed on a large number of chemicals and only a limited amount of time or money is available for assembling data, secondary sources are usually relied on. Certain types of information (e. g., annual production volume, $\mathrm{LD}_{51}$. octanol-water partition coefficients) are often tabulated in secondary sources. Other types of information (e.g., fate and transport in the environment, metabolic pathways) may be obtainable only from primary reports of research studies. These individual studies require considerable time and effort to locate, obtain, and review. In a multistage screening process, primary sources may be used only in the last stage, when the number of chemicals has been reduced to a small portion of the comprehensive initial list. In a very limited effort, primary sources may not be used at all.

\section{Assigning Priorities}

Two general methods used for assigning priorities to toxic chemicals are the ranking of individual chemicals [or classes) and the assigning of individual chemicals (or classes) to priority categories. Only 6 of the 32 lists surveyed are actually ranked. In most of the other lists, chemicals were 
either assigned to a single "high priority" category or distributed among several priority level categories.

Some numerical index or score is nearly always used for ranking and often used for assigning chemicals to priority level categories. When chemicals are to be ranked solely by parameters for which comparable quantitative information is available (e.g., oral $\mathrm{LD}_{50}$ in the rat or annual production volume) these data can be used directly. When the criteria for ranking cannot be defined in terms of parameters for which comparable quantitative information is available for all chemicals under consideration, chemicals are usually assigned scores. Scoring is also usually used when quantitative and nonquantitative factors are jointly considered for ranking.

The scoring process involves assigning numerical values to one or more parameters to produce an index or series of indices for each chemical. Indices can then be weighted and combined to produce a single score for each chemical,

Scoring can be totally subjective, totally objective, or somewhere in between, Subjective scoring involves the use of experts to assign priorities. The experts may be provided with information on each chemical or may simply be asked for their opinion. The credibility of subjective scoring will depend on the perceived expertness of panel members. Delphi techniques can be used to improve the validity or rankings, Scoring systems based on the Delphi method rely on the development of consensus among experts acting independently or as part of a panel. In the NSF Workshop Panel effort to select organic compounds hazardous to the environment, panel members identified chemicals of concern on the basis of their own experience and refined their judgments by considering available data for production, imports, uses, disposal, and toxicity. Through successive examinations by panel members, individually and together, in progressively greater detail, a priority list was developed. A weakness of such subjective scoring methods is their probable lack of reproducibility, which exists because different experts would be likely to reach different conclusions, (However, we know of no investigation of the reproducibility of such results in which different groups of experts were asked to assign scores to the same chemical s.)

Where scores can be assigned to specific values of parameters for which quantitative information is available, objective scoring may be preferable. Often, however, modifying factors may make the use of some degree of subjective judgment desirable in the assignment of scores even where quantitative information is available. For example, the octanol-water partition coefficient can be used as an index of bioaccumulation, but a more sophisticated scoring system would consider such other factors as chemical reactivity, metabolic fate, and vapor pressure of the compound.

The human health hazard posed by a chemical substance is a function of exposure to the substance and the inherent toxicity of the substance itself. Scores for various exposure and toxicity factors are usually combined for hazard-ranking purposes, No consensus has yet been developed, however, as to the optimum algorithm for hazard ranking in this way.

Linear weighting schemes are efficient and easy to use, and they have produced reasonable results. These schemes rely on the assignment of scores to a number of factors. Weights are assigned to each factor, and the weighted factor scores are summed to produce an overall score for the chemical. The choice of weight is usually a subjective judgment, involving consideration of both the importance of each factor and the reliability of the information used in assigning the score.

Multiplication of individual factor scores provides a wider spread of overall scores, Multiplication is frequently used with actual unscored quantitative data. This is especially likely where some of the data are in the form of fractions. For example, fraction of production lost would be multiplied by total production or fraction of dose absorbed would be multiplied by total administered dose. Multiplication cannot be used unless some correction is applied to allow for data gaps. In the development of one list, an algorithm for toxicity was applied which compensated for types of toxicities for which data were unavailable. The scores actually assigned to a chemical for various categories of toxicity were summed [T(total)]. The maximum toxicity possible for each of these categories for which data were available were also summed $[\mathrm{T}$ (possible)]. The ratio of these sums $[\mathrm{T}$ (total) $\div \mathrm{T}$ (possible)] was used as the toxicity factor in the hazard-ranking algorithm. The hazard-ranking algorithm used for ranking manufactured organic air pollutants was:$$
\mathrm{r} \times{ }^{\circ} \mathrm{pl} \times \mathrm{V} \times[\mathrm{T}(\text { total })-\mathrm{T}(\mathrm{possible})]=\mathrm{Sf}
$$$$
\text { where } \mathrm{P}=\text { score for annual production }
$$$$
\text { ipl score for fraction of production lost }
$$$$
\checkmark=\text { score for volatility }
$$$$
\text { I(total) = naximum toxicity score possible for those cate- }
$$
zories of toxicity for which data are available

$\Gamma($ possible $)=$ ium of maximum scores for each of the factors ised in compiling T(total)

if = inal scorefor hazard ranking
} 
In some cases the scores assigned to certain factors are logarithms of data or are selected subjectively in such a way as to approximate logarithms. In such cases, addition of the scores achieves the same result as multiplication of the actual data. Weighting of the factors before addition then corresponds to changing the base of the logarithm.

\section{Criteria}

The two general criteria used for assessments of health hazard are exposure and toxicity. Previous efforts to set priorities among toxic chemicals in the environment have been reviewed, and specific criteria used to assess the extent of exposure to these chemicals or the toxicity of these chemicals have been considered.

\section{Exposure}

The three aspects of exposure that are of greatest importance in hazard ranking are the number of people exposed, the frequency of exposure, and the quantities of the chemical to which they are exposed. Where direct information is available on these exposure factors, e.g., from monitoring of the ambient environment, of food, or of human tissue, it is obviously desirable to use this information. In most cases, however, we do not have direct information regarding the amount of a chemical to which members of a population are exposed, so that we must rely on indirect information, Several factors have been used, alone or in combination, as surrogates for direct measures of exposure.

Production.-The most commonly used index of exposure is the annual production volume. Since this information is quantitative, it is easy to use for scoring or ranking. In most cases, production volume does provide a rough indication of the amount of a chemical substance potentially available for release into the environment. However, the fraction actually released may vary over several orders of magnitude, from less than 0.001 for well-contained industrial intermediates to 1 for chemicals which are used dispersively.

For organic compounds produced by three or more manufacturers and produced in volumes greater than 5,000 $\mathrm{lbs}$, production information is compiled in the annual reports of the International Trade Commission and is easily available. production information can sometimes also be found in other literature sources or obtained from manufacturers or trade associations, but it is not easy to acquire and will be missing for a large number of chemicals. Production data for chemicals produced by less than three manufacturers are considered proprietary. In the near future, the EPA Toxic Inventory List, being prepared under section 8 of the Toxic Substance Control Act, may be a convenient source of production information, but at this time it is not clear how much of this information will be released to the public.

In addition to annual production volume, data on production capacity, transport volumes, imports, exports, net sales, or levels of consumption may sometimes be available. A major problem with using any of those figures as indices of environmental exposure is that these figures are based on commercial production and sales. The amounts of a chemical used as a captive intermediate are not included although such a chemical may be an occupational health hazard or a neighborhood pollutant in the vicinity of the plant. Very toxic and highly reactive chemicals are often manufactured at the same plant in which they are used. Manufacturing byproducts may never enter commerce but because they are waste products they are very likely to be released to the environment in plant emissions and in effluents. Toxic chemicals formed in the environment (byproducts of microbial degradation or photochemical reaction) also will not be reflected in production figures.

For naturally occurring chemical substances, such as heavy metals, the most relevant parameter is not production, but transfer from one compartment of the environment to another or conversion from one chemical or physical form to another. Such transfers are measures of the increase in the amount of the substance converted into a form in which it is potentially available for human exposure.

Occurrence in the environment. Actual measurements of chemicals occurring in the environment are available for only a small number of substances. Federal agencies tend to monitor on a regular basis only when required to by law. Air pollution measurements are available for carbon monoxide, total hydrocarbons, nitrogen oxides, sulfur oxides, total suspended particulate, and photochemical oxidants. Water pollution monitoring data are available primarily for pesticides, PCBs, and heavy metals. Monitoring information in Government data banks is often not retrievable in readily usable form. There may be a timelag of several years before a Federal agency summarizes, analyzes, and reports monitoring data. For qualitative or quantitative data on occurrence in the environment of chemicals not included in 
monitoring programs, it is necessary to search the literature for individual studies. Since these studies will usually report data for a small number of samplings at a limited number of locations (often one), they are difficult to use as a basis for the comparisons needed for ranking chemicals. The number of reported incidents of environmental occurrence will often be a function of interest in the compound rather than of actual frequency of occurrence.

An advantage of using this type of information, when available, is that it provides direct evidence that the chemical is an environmental contaminant.

Use. Patterns of use can be very useful indicators of potential for human exposure. A greater exposure risk is usually associated with dispersive uses than contained uses. Contained uses are those in which the chemical is not systematically released to the environment as a direct result of use (e.g., PCBs in transformers). In dispersive uses, chemicals are released to the environment as a direct consequence of use (e.g., pesticides, aerosols, and volatile solvents). The size of the population at risk is usually greater for consumer uses than for commercial or industrial uses. The class of the use (e. g., food additive, pesticide, or drug) will often determine which Federal agency has regulatory responsibility for a chemical. The use pattern will be a major determinant of the type, frequency, and amount of human contact with a chemical substance.

Unfortunately, accurate, complete, and up-todate use information is very difficult to acquire. Many industrial uses are considered "proprietary. "Uses can change rapidly so that information compiled in secondary sources is frequently out of date. One problem encountered in using use information is the difficulty of obtaining quantitative data. Since some uses are much more likely to lead to human exposure than others, a quantitative breakdown of uses would be particularly valuable. Even when use data are available, it is important to realize that not all human exposure to chemicals occurs during use. Exposure can also occur during production, formulation, and disposal or after environmental transformation.

Release. The rate at which a chemical substance is released into the environment can be a useful parameter in ranking toxic chemicals. Chemicals that are found in effluents or emissions are most likely to become environmental pollutants. Information on routes of entry of chemicals into the environment can in certain cases be essential to determining population exposure. In
1971 the sole U.S. producer of PCBs voluntarily restricted sales to those for closed-system applications. They assumed that this would minimize risk to human health and the environment. They overlooked the fact that most environmental release of PCBs occurs, not through use, but rather through disposal.

The quantity of a chemical released into the environment is usually unknown so that scores assigned for this factor must be based on estimates. The "release rate" is an interesting index recently developed by the NSF Workshop Panel to Select Organic Compounds Hazardous to the Environment. The release rate $(\mathrm{R})$ was defined as follows:

$$
\mathrm{R}=(\mathrm{P}) \mathrm{L}+(\mathrm{P}+\mathrm{I}) \mathrm{D}
$$

where $\mathrm{P}=$ overall annual U.S. production

$\mathrm{I} \quad=$ annual quantity imported

$\mathrm{L}=$ fraction of the production lost at plant site during manufacturing, conversion, and formulation

D $\quad=$ fraction of the material which goes to nonintermediate dispersive uses

Estimates of L and D are usually semisubjective, based on knowledge of the manufacturing process and on the likely breakdown of uses.

Environmental transport. A universal or widely dispersed contaminant will usually be given higher priority than a contaminant found only at specific locations or a contaminant whose entry into the environment results from a unique event or sporadic events.

For a persistent chemical, the distinction between a local, widely dispersed, or universal contaminant may be a function of time. Unexpectedly wide transport has been observed in recent years for chemicals such as DDE and PCBs, PBBs entered the environment as a result of a single accidental occurrence and contaminated a small number of Michigan farms. Since these chemicals are persistent and have entered the soil, water, and food supply, they will continue to disperse.

Substances that appear to be relatively innocuous or inert at their point of release may be transported to segments of the environment where they have significant adverse effects. Useful models for the reliable prediction of the movement of chemicals in the environment would be extremely valuable for setting priorities among toxic chemicals for purposes of monitoring, control, or regulation. Such information would also be valuable in determining which segments of the environment should be monitored or controlled. Unfortunately, although models are available for problems such as plume dispersion for stack emissions, there are no generally accepted, easy to apply, widely used 
models for environmental transport. Moreover, realistic models of environmental transport are usually extremely complex, and it is impracticable to use them in priority-setting exercises.

Persistence. Chemicals can be transformed and degraded in the environment through chemical, photochemical, or microbial processes. The relative importance of these processes will be influenced by the compartment of the environment with which we are primarily concerned. Reactions leading to simpler compounds which are part of a series leading ultimately to the free elements or elemental oxides are considered to represent "degradation," Other reactions lead to "secondary pollutants.

Persistent chemicals are often given priority over easily degraded or transformed chemicals in hazard assessment systems, on the assumption that persistent chemicals are more likely to spread through the environment and will continue to accumulate as long as they are released. When persistence is used as a criterion for hazard assessment, it is important to be aware, however, that there are cases in which degradation products or secondary pollutants are more toxic than the chemical substances initially released,

Biodegradability. Although all living things can play a role in transformation of chemicals in the environment, the lead role is attributed to microorganisms. The role of micro-organisms in transforming chemicals is extremely important in the lithosphere and also important in the hydrosphere. Micro-organisms are frequently efficient at metabolizing natural chemicals in their environment. Industrial chemicals are inherently less likely to be rapidly degraded by micro-organisms. Parameters often used as indices of biodegradability are the biochemical oxygen demand (BOD) or chemical oxygen demand (COD), Estimates of biodegradability can sometimes be based on chemical structure.

Chemical reactivity. The assessment of the chemical reactivity of a compound is often based on known physical and chemical properties such as:

- physical state,

- vapor pressure,

- solubilities,

- auto-oxidation propensity,

- redox potential,

- acid-base characteristics,

- rate of hydrolysis, and

- rate of photochemical degradation.

One parameter which can-be used for persistence in the environment is half-life, the time re- quired for removal of half of the molecules of a given compound from the environment or from a specific compartment of the environment. Halflife in the atmosphere, for example, would be a function of such properties as photoreactivity, reactivity towards active forms of oxygen, water volubility, volatility, and adsorption to fine particulate.

Bioaccumulation. Bioaccumulation can be defined as the long-term presence of a xenobiotic substance in living tissue at concentrations significantly higher than those in the surrounding environment. Chemicals that bioaccumulate are often given priority over other chemicals in hazard-assessment schemes. Concentrations of chemicals that bioaccumulate are likely to increase along a food chain, a process referred to as "biomagnification. " For such chemicals, residue levels tend to be higher in herbivorous animals than in plants, higher in carnivorous animals than in herbivores, and highest in carnivores that eat other carnivores. Since humans may eat organisms in any of these categories and since chemicals which bioaccumulate will usually be stored in human tissues, if such chemicals have any toxic properties they may pose a higher risk to human health than would be predicted on the basis of environmental concentrations alone.

Since bioaccumulation is often associated with a high lipid volubility relative to water volubility, the partition coefficient can be used as an indicator of the tendency to bioaccumulate. The partition coefficient is a measure of the distribution of a solute between two immiscible liquid phases in which it is soluble. For a single molecular species, the partition coefficient is a constant and does not depend on relative volumes of solutions used. The most commonly used coefficient indicates relative volubility in organic and aqueous phases, providing information on the hydrophobic or hydrophilic nature of the compound.

Although absorption and accumulation of toxic pollutants are related to their partition coefficients, partition coefficient alone does not determine bioaccumulation. Other characteristics of the chemical (reactivity, vapor pressure) and of the organism (metabolic pathways, nutritional status, fat content of body tissue) will also influence the extent of bioaccumulation. Active accumulation of a substance that resembles another chemical is frequently predictable from the properties and structures of both,

For inorganic compounds relative volubility is not the primary factor in determining extent of storage in body tissues. Passive accumulation 
through formation of complexes with organic ligands, especially those containing sulfhydryl and amine groups, can also occur. The synthetic compounds most likely to accumulate in this way are organometallic chemicals. The tendency of a chemical to accumulate in this way can be predicted from knowledge of the likelihood of the chemical to form complexes with natural bases.

Population at risk. The size or type of population exposed may be considered as a factor in setting priorities for toxic chemicals. Types of populations at risk could be:

- the general human population,

- population of a geographical region,

- population of a specific neighborhood,

- population in the immediate vicinity of an industrial plant,

- people whose diets contain a high amount of particular foods,

- occupational groups, and

- highly susceptible groups.

Population groups can be further classified according to whether their exposure is voluntary or involuntary. Priority considerations should be given to chemicals to which there is extensive involuntary public exposure or to which susceptible segments of the population are exposed. Some ranking schemes consider certain segments of the population more 'valuable' than others (e.g., the young more "valuable" than the old). This approach is highly subjective and ethically questionable.

\section{Toxicity}

The toxicity of a chemical is the second determinant of hazard. The difficulties encountered in estimating the biological activity of a chemical are as severe as those encountered in estimating exposure. Here we find ourselves at "the frontiers of scientific knowledge" in attempting to evaluate the significance of laboratory data for human experience.

Most priority-setting efforts have scored chemicals on a series of toxic effects, There are, however, some general indices of toxicity, such as the threshold limit values for atmospheric exposure in the workplace established by the U.S. Department of Labor and the American Council of Governmental and Industrial Hygienists, tolerance levels for pesticides in food set by EPA, or "action levels" for food contaminants set by FDA. These indices are based on reviews of a range of toxicity data and represent attempts to identify threshold levels for hazard or for lack of assurance of safety. Although the reliability of such indices as precise measures of degrees of hazard is debatable, where available they are useful in prioritysetting exercises because they represent an attempt to weigh all toxicity data available at the time when they were established.

Metabolic factors.

1. Uptake and absorption.-Even if the amounts to which people "may be exposed could be determined, we still would not know the amount that could be expected to enter the human body and cause biological damages. The term "penetrability" is sometimes used to represent that portion of the chemical to which the person is exposed that is expected to be absorbed into the body, This factor includes consideration of both the routes of exposure and the fraction of the chemical absorbed after exposure by each route,

2. Biotransformation. -The metabolic conversion of a chemical within the human body should be considered, as far as possible, in assessing potential health effects. A chemical can be detoxified, activated, or converted to a compound with different biological properties. The metabolic pathway can be dosedependent.

3. Elimination. - The turnover rate in the human body can be an important factor in assessing biological effects. If, however, a chemical has been scored for bioaccumulation as an exposure factor, it may not be necessary for it to be scored for turnover in humans, as these factors are closely related,

Absorption, bioconversion, and elimination are complex factors and are difficult to handle for ranking purposes, Information is not readily available and is rarely compiled in easy-to-use secondary sources. For many chemicals, no metabolic information is available. When metabolic studies are found, they are often reported in qualitative rather than quantitative form. Relating metabolic information to adverse health effects often requires a high level of expertise in toxicology. Despite all these problems, a toxicological ranking system which takes into account these metabolic factors is more sophisticated than and usually superior to a system which does not,

Acute toxicity. The biological effect of a chemical depends on the quantity of the chemical with which the organism must deal, Chemicals having adverse effects at low dosage would be considered more toxic than chemicals having similar effects only at much higher dose levels. It is difficult to perform this ranking at low effect levels. The 
effects of low dosages are often subtle. Direct experimental estimation of the level affecting 1 percent of the population may require several hundred animals to obtain adequate statistical precision. For these reasons, lethal dosage levels are usually used in ranking chemicals for acute toxic ity.

A common parameter of acute toxicity is the LD $D_{\text {in }}$, the dose of a substance at which half the test animals would die. To be comparable, results should be based on animals of the same species, strain, sex, and age. The same route of adminis$\mathrm{t}$ ration should be used.

Other commonly used parameters of acute toxicity based on lethal doses are:

$L_{\text {i11 }}=$ the concentration which is lethal to half the test population. The duration of exposure should be specified. LC is usually used for concentration in air, but can also be used for concentration in the ambient water to which aquatic organisms are exposed

$\mathrm{LD}_{1}$, = the lowest reported lethal dose

$L_{1,1}=$ the lowest reported lethal concentration

Available data on these parameters for 16,500 different chemicals are summarized in the Registry of Toxic Effects of Chemical Substances published by NIOSH. The major objection to acute lethality as a basis for ranking environmental contaminants is that the acute lethal doses are very much higher than those levels one would expect to find in the environment. Furthermore, values for acute lethality can vary widely between species. Use of this parameter can be justified, however, on the basis that the $L D^{\prime \prime \prime}$ is at least a crude index of biological activity. It is reasonable to consider a chemical $\mathrm{w}$ i $\mathrm{t} h$ a very low $L D_{n 11}$ to be a relatively toxic chemical. on the other hand, a chemical with a high $L_{5}$ is not necessarily safe, since long-term exposure a t low concentrations may have such adverse effects as carcinogenicity or damage to the reproductive system.

Data on acute toxic effects other than lethality are less frequently compiled, are more difficult to use for ranking purposes since they arc not usually reported in numerical form, and may not be indicative of effects occurring at the low dosage levels ordinarily found in the environment. Nevertheless, nonlethal acute effects can provide useful information about target organs and should be considered as part of the overall picture when subjective judgments are made.

Carcinogenicity. In principle, it would be useful to categorize chemicals as strongly carcinogenic, moderately carcinogenic, weakly carcinogenic, co-carcinogenic, or having no neoplastic effects. There is usually not enough information available, however, to do this. Dose-response curves are rarely determined for carcinogens. Negative results in carcinogenicity tests are usually not accepted as proof of noncarcinogenicity. Comparisons between carcinogenic "potency' of various chemicals have been made on the basis of percent age of a test population developing malignant tumors, the number or types of species in which positive results have been reported, the types of tumors observed, the lowest dosage causing malignancy, or the lag time between administration of chemical and observation of tumors.

Chemicals are sometimes classified with respect to the degree of certainty of their carcinogenicity (e. g., known carcinogen or suspected carcinogen). EPA has recently attempted to order the NIOSH list of suspected carcinogens according to the relative degree of concern that might be warranted regarding possible human carcinogenic potential. A four-digit code was used. The first digit represented the species in which carcinogenic response was reported. The second digit designated the number of different species for which a carcinogenic response was reported. The third digit was assigned on the basis of route of administration. The last digit was a count of the number of different species-route combinations.

The most complete source of data and references related to chemical carcinogenesis is the Public Health Service's Survey of Compounds Which Have Been Tested for Carcinogenic Activity. A master index of the series is maintained on tape at the National Cancer Institute headquarters.

Evaluations of carcinogenic risk are made by the International Agency for Research on Cancer under the auspices of the World Health Organization. They do not use a formal ranking system.

Mutagenicity, A mutation is defined as any heritable change in genetic material. The results of mutations are usually undesirable and may include abortion, congenital anomaly, genetic disease, lowered resistance to disease, decreased lifespan, infertility, mental retardation, senility, or cancer.

The reliability and accuracy of the prediction of mutagenicity in man from the ability of a chemical to produce mutations in experimental tests systems is controversial. Many types of tests are currently available for use as indicators of mutagenic potential. Some (e. g., the Ames test, the dominant lethal test) are actual tests for mutage- 
nicity. Others test for a variety of events relating to, or at least correlated with, mutagenesis. Some such tests are for chromosome aberrations, DNA damage or repair, increased rate of sister chromatid exchange, or other phenomena considered to be relevant to mutagenic potential. Because even those tests that actually demonstrate mutagenicity are highly specific for detecting a single type of mutation, results of a battery of tests are desirable for an evaluation of mutagenic potential of a chemical substance,

Structure-activity relationships have met with limited success in prediction of mutagenicity. Many chemical mutagens (but not all) act as electrophiles permitting a prediction of mutagenic potential of compounds that might form a reactive electrophilic species.

Many difficulties are encountered in extrapolating to man results obtained in microbial, mammalian cell culture, or test animal systems. Potential for mutagenic effects in man should be assessed only after results of testing in various experimental systems are considered in conjunction with other relevant factors such as chemical structure, biochemical activity, and metabolism in man.

Teratogenicity. Certain chemicals, when administered to a pregnant female, can interfere at a critical stage of embryogenesis or organogenesis and can cause embryonic death or malformation, A chemical with such properties is a teratogen. Testing for teratogenicity should ideally involve administration of the test substance only at the period critical for organogenesis of the system sensitive to the teratogen. Sensitive periods of organogenesis are characteristic of particular chemical teratogens.

At the present time, only tests in pregnant mammals are generally accepted by scientists and regulatory agencies as valid tests for teratogenicity, because the maternal-placental embryonic relationship cannot be duplicated in submammalian test systems. Other test systems sometimes used as indicators of teratogenic potential include bacteria and other unicellular organisms, somatic cells in culture, tissue culture, organ culture, intact invertebrate embryos, cul- tured mammalian embryos, and incubating chick embryos. Of these systems, chicks and to a lesser extent fish are the most widely used,

It is difficult to rank chemicals on the basis of teratogenic potency. The type of dose-response data needed for quantification of teratogenic risk is seldom available. Interspecies extrapolation is poorly understood. There is an enormous range of variability of teratological end points. Chemical structure has been found to be of little use in predicting teratogenicity.

Other toxic effects.

1 , Effects observed in humans, - Reports of toxic effects in humans can be based on epidemiological or clinical observations. There are severe problems in interpreting this type of information. Humans are rarely exposed to only a single chemical substance, It is usually difficult to find control groups with zero exposure to an environmental contaminant. Results based on occupational exposure may underestimate risk, because susceptible segments of the population (infants, children, the aged, the chronically ill) are excluded from the workplace. When workers become sick they often stop working and are no longer part of the occupational cohort. Another problem in interpreting human data is that the doses are often unknown.

2. Chronic toxicity, -Biological effects of a chemical administered in low doses over an extended period of time may be quite different from acute effects. Chronic exposure test protocols are more relevant than are acute protocols to the types of exposure due to environmental contaminants.

Because of the wide range of end points observed during chronic exposure tests, comparisons are difficult to make. Comparing one type of effect with another (for example, comparing impaired fertility with liver damage) on the basis of severity is highly subjective. Ranking chemicals on the basis of chronic toxicity therefore requires a high level of scientific expertise. 


\section{Approaches to Monitoring Organic Environmental Contaminants in Food*}

by J ohn L. Laseter, Ph.D.

\section{INTRODUCTION}

Trace quantities of potentially toxic organic compounds are frequently found in the environment. These compounds sometimes possess properties that may have teratogenic, mutagenic, and even carcinogenic effects on humans and animals. Some of the compounds, such as pesticides, have been intentionally released into the environment (1), while others have found their way into the environment by accident, through carelessness, or as byproducts of industrial processes. Many of these compounds are subject to breakdown in the environment as a result of both physiochemical and biological processes, such as chemical weathering, photodecomposition, metabolism, and biodegradation by micro-organisms $(1,2)$. In many cases, it is not uncommon for the breakdown products to have greater health effects on humans and animals, than the parent compounds.

Because of the potentially toxic properties of so many of these compounds and their breakdown products, several pieces of Federal legislation have been enacted in recent years to monitor, evaluate, and control the amounts of the pollutants in order to protect our health and environment. As a result, monitoring (screening) programs have been established for many types of organic pollutants in the environment. All of these programs rely heavily on the ability of analysts to correctly identify and quantify these compounds at the parts per million (ppm) and parts per billion (ppb) levels in a variety of sample matrices (3). In order to successfully obtain useful information at these concentration levels, it is essential that methods which are sensitive and selective be developed and used. Whatever method is ultimately

* For purposes of this document, food represents all solid. sem isol id, a nd liqu id forms of food products. including bott lext water, consumedbuman.

'I he terms "momitor ang" and "sceening" areused in terchangeably $n$th is document. selected, it is imperative that it provide unequivocal results.

Most methods for monitoring and for the analysis for trace levels of organic pollutants in the environment necessarily consist of several steps These include sample collection and storage, sample workup, and component identification and quantification.

The collection and storage of samples is an important phase of an analytical method if meaningful interpretation of the data obtained is to be achieved. The sample selected for analysis must be representative of the whole system being examined, and must be free of contamination due to improper collection and handling techniques. Once collected, the samples must be stored under conditions that will reduce or eliminate changes in their composition.

Another important phase in an analytical method is the sample workup. During this stage, the sample usually undergoes an extraction process whereby the compounds of interest are removed from the sample matrix. Organic solvents, and in some cases inert gases, are usually employed in the extraction process. Because the extraction process is seldom very selective, many organic compounds in addition to those of interest are also extracted. In order to reduce the amounts of these other compounds that may interfere with the analysis, a sample cleanup procedure usually follows the extraction process.

The last and most difficult phase of an analytical method is the identification and quantification stage. The identification process is often accomplished by comparison of the physical and chemical properties of the unknown compound against the same properties of an authentic standard compound. For complete unknowns, the identification process can be very difficult. The quantification process can only be accomplished after the unknown compound has been identified and usu- 
ally involves comparison of the detector response for the compound of interest against the detector response for known quantities of an authentic standard.

In the analysis for trace levels ${ }^{2}$ of organic compounds in the environment, it is often very difficult to obtain accurate and reliable qualitative as well as quantitative results. This is evident by the countless examples in the literature of errors in both qualitative identifications and quantitative estimations of trace quantities of many organic compounds in the environment (5).

Of the many methods currently available for the qualitative identification and quantification of organic compounds, few are sensitive and specific enough for meaningful trace analysis. Table G-1 summarizes the techniques for organic analysis and some of their advantages and disadvantages $(4,6)$.

The most common techniques in use for trace organic analysis are gas chromatography with the use of selective detectors (7) such as the electron capture (EC). Hall electrolytic conductivity, and flame photometric detectors; high performance liquid chromatography (HPLC) (8); and combined gas chromatography-mass spectrometry (GC-MS). Selective detectors for gas chromatography are necessary because environmental samples are often very complex and the selectivity of the detectors simplifies the analysis by allowing only certain compound classes to be detected at any one time. The most powerful of these techniques is the GC-MS technique $(4,6,9-11)$, since it not only provides qualitative information of nanogram quantities of single compounds present in the sample, but also provides information which can be used for quantification of individual components in the sample.

A more recent technique involves the combination of liquid chromatography and mass spectrom-

For purposes Of this document. " rare levels" is definedas a concentrat ionbelow the low parts-per-m ill ion level. Detection at these concent ra t ions is important becatuse many organics arebiologica 1 lvac tive even at the pa rts-per-trillionlevel. etry (12-14). This technique expands the area of trace organic analysis to the identification and quantification of compounds that are not suitable for analysis by gas chromatographic techniques.

\section{Table G-1 .-Techniques Available for Qualitative and Quantitative Organic Analysis}

\begin{tabular}{|c|c|c|}
\hline Method & $\begin{array}{l}\text { 4pproxımai } \\
\text { detection } \\
\text { lımıt.gm }\end{array}$ & $\begin{array}{l}\text { Specificity or } \\
\text { common uses }\end{array}$ \\
\hline \multicolumn{3}{|l|}{ Gas chromatography } \\
\hline Retention indices... & $\begin{array}{c}10^{-10} \\
\left(\mathrm{H}^{2} \text { flame }\right)\end{array}$ & $\begin{array}{l}\text { Detects most } \\
\text { compounds }\end{array}$ \\
\hline Electron capture ... & $10^{-12}$ & $\begin{array}{l}\text { Halides, conjugated } \\
\text { carbonyls. nit riles. di- } \\
\text { and trisulfides }\end{array}$ \\
\hline Flame photometer. . & $\begin{array}{l}10^{9}(\mathrm{~S}) \\
10^{-11}(\mathrm{P})\end{array}$ & Phosphorus, sulfur \\
\hline $\begin{array}{l}\text { Nitrogen/ } \\
\text { phosphorus ...... } \\
\text { Chemical methods }\end{array}$ & $10 \cdot 12$ & Nitrogen, phosphorus \\
\hline Pyrolysis ......... & $10^{9}$ & $\begin{array}{l}\text { Compound type } \\
\text { determination }\end{array}$ \\
\hline Chemical reagents. . & $10^{6} 6$ & $\begin{array}{l}\text { Classical functionality } \\
\text { determination }\end{array}$ \\
\hline Electrolytic systems & $10^{-8}$ & $\begin{array}{l}\text { Sulfur, nitrogen, } \\
\text { halogens }\end{array}$ \\
\hline \multicolumn{3}{|l|}{ Instrumentation } \\
\hline Infrared-grating & $10^{-6}$ & $\begin{array}{l}\text { Lompound category type } \\
\text { identification }\end{array}$ \\
\hline interferometer. & $10^{-7}$ & $\begin{array}{l}\text { Compound category type } \\
\text { identification }\end{array}$ \\
\hline Ultraviolet ........ & $\begin{array}{l}\text { Variable } \\
\text { to } 10^{-10}\end{array}$ & $\begin{array}{l}\text { Aromatics conjugated } \\
\text { carbonyls }\end{array}$ \\
\hline $\begin{array}{l}\text { Proton magnetic } \\
\text { resonance... }\end{array}$ & $10^{\cdot 5}$ & $\begin{array}{l}\text { Excellent for function. } \\
\text { some molecular } \\
\text { weight data }\end{array}$ \\
\hline \multicolumn{3}{|l|}{ Mass spectrometer } \\
\hline Batch inlet ...... & $10^{-7}$ & $\begin{array}{l}\text { Best for complete } \\
\text { identification, }\end{array}$ \\
\hline GC.MS mode ... & $10^{-11}$ & $\begin{array}{l}\text { molecular weight } \\
\text { structure, and }\end{array}$ \\
\hline $\begin{array}{l}\text { Multiple ion } \\
\text { detection . }\end{array}$ & $10^{\cdot 12}$ & $\begin{array}{l}\text { function Confirm any } \\
\text { compound }\end{array}$ \\
\hline
\end{tabular}

SOURCE Adapted from W Mctadden. "lechniques or Combined Lias Cnromatography:Mass Spectrometry "Wiley-Interscience. New York. NY 1973, p. 4 and Trace Organic Analysis. "Environmental Science and Technology. 12,757 i1978 


\section{ANALYSIS FOR EPA PRIORITY POLLUTANTS IN FOODS AND WATER}

The recent EPA/NRDC consent decree established an analytical procedure for the analysis of 129 priority pollutants (chemical indicators of organic pollution) in industrial waste water $\left(\begin{array}{ll}1 & 5\end{array}\right)$. Of these, 15 pollutants are metal, with the remainder being individual organic compounds and compound classes. The procedure for the analysis of the organics is strictly a GC-MS computer method designed to provide qualitative as well as quantitative information about the presence of the priority pollutants in waste waters.

Basically, the procedure requires that four separate analyses be performed on the sample. These are an analysis for the more volatile organics, an analysis for the basic and neutral extractable organics, an analysis of the acidic extractable organics, and an analysis for organochlorine pesticides. The analysis for volatile organics is usually accomplished by a vapor-stripping lechnique more commonly called the purge and trap technique, whereas all other analyses involve liquid-liquid extractions, and subsequent injection into a GC-MS system.

The procedure for waste water analysis can easily be applied to water and other liquids including foods: however, some modifications of the EPA procedure are necessary. This can be accomplished, since techniques similar to those developed for the analysis of tissues (16-18), can be adapted for foods. However. because of the greater complexity of the sample matrix, the resulting extracits usually contain large quantities of high-molecular weight organics that must be removed in order to achieve analysis of the individual trace organics. This removal process can be accomplished by one of several cleanup methods which are available, such as, liquid-liquid partitioning. chemical digestion, thin-layer chromatography (TLC), and liquid chromatography (LC). Whatever method is selected, it must be genthe enough to preserve the composition of the mixture of the trace organics, it must have a high recovery of the compounds of interest, and it must be quick and reproducible. Once the extraction and cleanup stages are complete, the remaining sample can be divided into different fractions and treated according to the methodology prescribed for the waste water analysis. The general analytical scheme is illustrated in figure G-1. Figure G-2 shows the general scheme modified for analysis of

\footnotetext{
As noted in the revisent April 1977 HPA document.
}

food samples. An estimate of the space, manpower, and cost associated with the analysis of the present 114 organic EPA priority pollutants in food and water samples is given in table G-2. It should be pointed out that considerable research and development effort must be expended to adopt the methods proposed to foods in general.
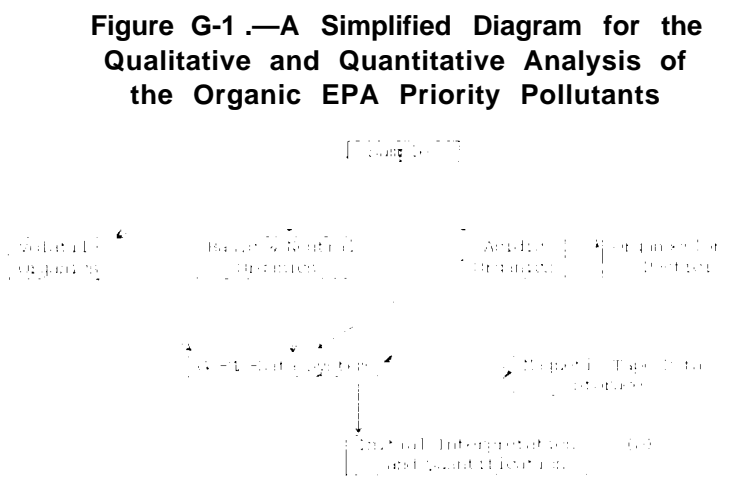

NOTES

St toods. this procedure requires modification. See figure G.2

Identification is based on the presence of characteristic ion fragments and as sociated chromatographic retention lime data Absolute identification would require a detalled interpretation of the complete mass spectrum of the organic compound of interest

Figure G-2. - A General Scheme for the Qualitative and Quantitative Analysis of the EPA Organic Pollutants in Semisolid Foods

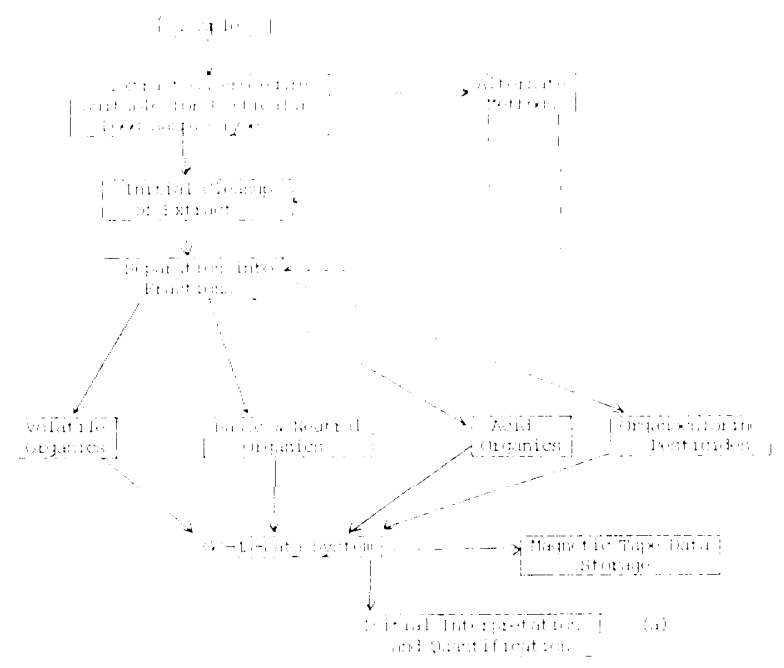

JTE ldentification is baspon on the presence of characteristic ion fragments and associated chromatographic metention time data Absolute idem Iffication woule regure at detaled interpretation of the complete mass spectrum of the? argate compount of interest. 
Table G-2.-Estimated Space, Manpower, and Cost Associated With the Analysis of Priority Pollutants in Tissue and Water Samples According to EPA Analytical Protocol ${ }^{a}$

Category

A. Instrumentation . . . . . . . . .

B. Space. . . . . . . . . . .

C. Downtime for instrumentation

D. Minimum assignable . . . . . . manpower
Response and/or cost

GC-MS data system with automated liquid injection device.

$-\$ 200,000$ each

$1,500 \mathrm{ft}^{2}$ equipped with typical laboratory facilities and furniture including adequate air-conditioning.

$30 \%$ (this figure can vary from 20 to $50 \%$ as a function of staff experience and logistics support).

Ph. D or equivalent - 1

M.S. or equivalent - 1

B.S. or equivalent - 2

Total

Estimated operational cost per routine sample $-\$ 2,000$ to $\$ 2,500$ (Scheme One as illustrated in figure G-I).

Estimated operational cost per routine sample $-\$ 2,500$ to $\$ 3,000$ (Scheme Two as illustrated in figure G-2, as a function of cleanup difficulty).

$\mathrm{a}_{\text {It }, \mathrm{s}}$ assumed that the laboratory has other ongoing activities and further that samples would be analyzed on ly once and In succession The ultimate size and cost of such a monitoring facilityıs based on processing only a few hundred samples per year No capital costs are calculated into the operational cost per sample

b Typicallaboratory facilities and furniture Include laboratory benches tables with sinks shelves forreagents and equipment. vented hoods, refrigerators, laboratory balances and scales, $\mathrm{pH}$ meters, hot plates, laboratory glassware, c hem ical, etc This Includes special toxic chemical and carcinogen processing fac ilities Laboratory space of the type desc ribedabove cost on the order of $\$ 100 / \mathrm{ft}^{2}$

$\mathrm{c}_{\text {Manpower requirements are not Included for maintenance and logistic suPPort }}$

\section{SCREENING FOR UNSPECIFIED POTENTIALLY TOXIC COMPOUNDS AND CHEMICAL CLASSES IN FOOD AND WATER}

There is a possibility that other compounds or classes of compounds not included in the EPA priority pollutants list will find their way into the environment. For this reason, it is essential that some form of monitoring system be established that will look for the appearance of particular compounds or classes of compounds in food and water over a period of time. A recent National Research Council report on environmental monitoring (20) recommends the establishment of new monitoring programs to anticipate pollution problems and to discover environmental pollutants in their early stages of development so that appropriate corrective measures can be implemented before the problem becomes unmanageable, or worse, irreversible. A typical monitoring program is the EPA Mussel Watch Program (2 I), the EPA National Pesticide Monitoring Program, and the National Pesticide Monitoring Network for Birds operated by the U.S. Fish and Wildlife Service.

In establishing a monitoring program, the type of compounds to be monitored would be selected as candidate compounds on the basis of their chemical class, their use, and their suspected toxicity, These candidate compounds might include steroids, phenols, amines, halogenated organics, polynuclear aromatic hydrocarbons, and any newly appearing organic that illustrates a marked increase in concentration over a period of time.

The monitoring program design would involve the establishment of appropriate sampling and sample-handling guidelines, together with the modification of currently used analytical procedures to include the new compounds or classes of compounds. A typical monitoring program would involve the use of high-resolution gas chromatographic techniques using the universal flame ionization detector (FID) capable of detecting traces of known candidate compounds, and computer techniques that allow for rapid comparisons of samples to establish trends. With the use of high-resolution gas chromatography (employing glass capillary columns"), one can readily separate in an environmentally derived sample,

'Glass capillary columns reflect the state-of-the-art of gas chromatography, and are superior to the conventional packed columns of vears past. For example, glass capillary columns are generally about 10 to 12 times more effective in separating complex mixtures. 
several hundred organic species, the vast majority of which have not been characterized.

The analytical program would involve a preliminary screening to establish baseline levels of the candidate compounds in samples of foods and water, or selected indicator species over a given period of time, followed by periodic screening of similar samples to determine changes or trends with time. If alarming trends are observed, corrective actions and measures can be implemented.

Of the techniques available for analysis, the methods best suited for the class or classes of compounds under consideration would be selected for routine monitoring. These would include high-resolution gas chromatography or high-performance liquid chromatography and supporting computer methods which would include the use of internal and external standards, and use of pattern recognition techniques. ${ }^{5}$ The monitoring would be set at a concentration level below the actual legal accepted level (for example, $0.01 \mathrm{ppm}$ or one-tenth of the action level if it is known),

Should the screening result in an observation of an increase in levels of either unknown or selected compounds or classes of compounds, suggesting the entry into the food or water of new materials, additional analytical efforts would be employed to attempt characterization of the new compound(s) observed. Preliminary information would be transmitted to associated toxicologists for evaluation and comparison with information available on known toxic compounds.

Pattern recognition technigues are "data-interpretation processes developed by empirical examination of data from known sources, to elucidate relations from unknown data. "(22)

\section{LABORATORY REQUIREMENTS}

Because organic analysis at the submicrogram (ppm and ppb) levels is a complicated and difficult process requiring sophisticated instrumentation and expertise, the laboratory selected to perform analyses for trace organics in foods and water must be equipped with state-of-the art technology and experienced personnel. The best setting for such a laboratory is in a location where it can establish ties with the R\&D community where experts in a variety of disciplines can be found who can serve and participate as consultants at various levels. It should be pointed out that analytical techniques may require modifications to upgrade the technology and a substantial expenditure of funds to keep the program operating at maximum efficiency and information output.

The typical nationwide monitoring program would have several regional centers, each wellequipped with the appropriate instrumentation and personnel to perform the analyses and conduct the program. In addition each center would have a review board composed of senior scientific personnel in such fields as toxicology, analytical chemistry, environmental chemistry, etc., to assess and interpret the data developed. To facilitate the handling of all the data acquired, each center would be equipped with data-archiving facilities for both GC, LC, and GC-MS data and dataprocessing methods that would allow for rapid retrieval, comparison, and evaluation of these data, Some of these data-handling techniques are now available and others are under development (23-26] for such a central data management system (see table G-3). Figure G-3 illustrates a proposed analytical scheme to monitor for the appearance of unknown organics in addition to the current EPA priority pollutants in food and water samples.

In support of the nationwide monitoring program would be a quality control laboratory which would coordinate intercenter calibrations, and would spot-check and confirm selected data developed by the regional laboratories. Of necessity, the quality control laboratory would be better equipped than the regional centers, so that it could resolve the problems and issues that can occur during routine analyses. A minimum of 10 percent of all samples analyzed by the regional centers would be confirmed by the quality control laboratory, Additionally, a library of samples used in the actual analytical studies would be stored for future reference. 
Table G-3._Estimated Space, Manpower, and Cost Associated With the Monitoring of Unknown Potentially Toxic Compounds in Addition to the EPA Priority Pollutants
Category

A Instrumentation

B Space

C Downtime for instrumentation

D Minimum assignable manpower operational cost per routine sample

\section{E Estimated}

Response and/or cost

a Small, high throughput GC-MS data-system with automated liquid injected device $-\$ 50,000$.

b $\mathrm{El} / \mathrm{Cl}$-equipped high-resolution MS-data system with automated injection device $-\$ 200,000$ to $\$ 300,000$

c GC-FID-EC system $\$ 15,000$ (additional chromatographic systems may be required)

d LC Interfaced into MS system $-\$ 20,000$

e Central data management system $\$ 750.000$ to $\$ 1.000 .000$

f. Cold storage and processing facilities $\$ 200.000$

-5.000 to $6.000 \mathrm{ft}^{2}$ equipped with typ ical laboratory facilities and furniture including adequate air. conditioning.

$30 \%$ (this figure can vary from 20 to

$50 \%$ as a function of staff experience and logistics support)

a. Direct

Ph.D or equivalent - 2

M.S. or equivalent - 3

B.S. or equivalent - 3

Total 8

b. Indirect support

Ph.D. or equivalent - 2

B.S. or equivalent - 2

Total 4 b

- $\$ 3.000$ to $\$ 4.000$ per sample if data are routine. The cost per sample which has new components and requires extensive characterization is difficult to estimate. but would certainly be greater than the cost per routine sample. For example. cost may exceed $\$ 10,000$ per individual compound.
Typical laboratory facilities and furniture include laboratory benches. tables with sinks. shelves for reagents and equipment, vented hoods, refrigerators, laboratory balances and scales. $\mathrm{pH}$ meters. hot plates, lanoratory glassware. chernicals, etc. This includes special toxic chemical and carcinogen process ing facilities.

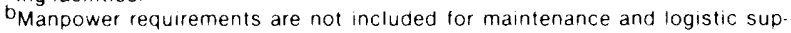
port.

NOTE 1: It is assumed that the laboratory is not involved in toxicological and human hazard evaluation and the general data system hardware is available. However. Only a portion of the required software can presently be employed and $R \& D$ work $i$ mains to be done to make the entire proposed system functional (22-25)

NOTE 2: The size and estimated cost of such a monitoring facility is a function of the numbers of samples to be processed per year At this time it is impossible to estimate the number of indepth characterizations may be necessary per year. The above described lacilty is designed may be necessary per year. The above described lacilly is designed to process only a few nundred samples per year No capital costs are calculated into operation cost per sample.

\section{Figure G-3.-General Analytical Scheme To Detect and Monitor New Trace Organics and Priority Pollutants in Food and Water Samples}

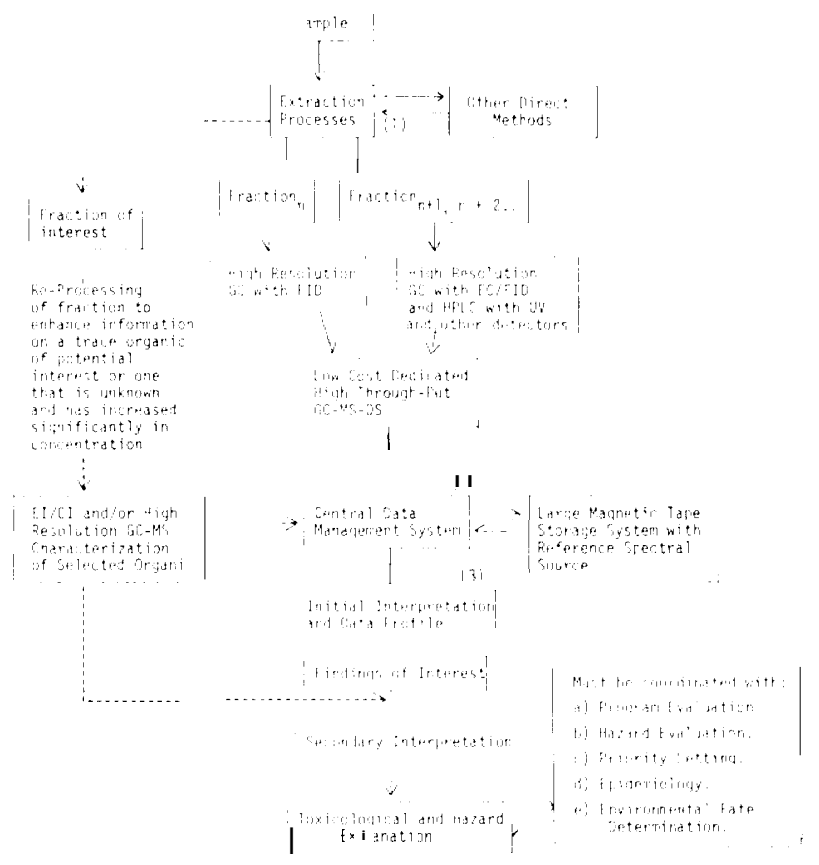

NOTES

1 A variety of $f$ raclionat 1011 andisolationprocedures may be employed in addi t ionto I iqu iell igu Id ext ract ton

2 Central data system would conduct the following activities

- index and store all raw data on magnetic tape or disc

- calculate a retention index for each unresolved component

- compute changes with turne? for each resolved component and estimate - normal concontration variations per unit lime

- llag variations outsule expected threshold values andior thes appearance of a newly resolved component.

- contuct wattern recogrtion techniques for new groups or species of organics

- store all primary and secondary interpmetations of finds. qualitative and quantitative fondungs and information for toxicological and hazard evalua tion

- cross correlate mass spectral data of known compounds with gas chromatographic data for unresolved unknowns

3. A cumpleter mass spectrometric evatuation of each traction from a given sam Whe type or location would be accomplished initially to establish the identity of resolved components and followed by analytical exercises designed to provite intormittion on speteiffe components

4. Prelumnary interpretations of data woukd be designed to pick out the presence of new combounds or an increase in concentration of those

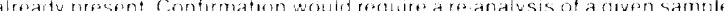




\section{DEVELOPMENTS THAT WILL IMPACT LOW CONCENTRATION ORGANIC MONITORING}

Of all the developments in technology for chemical analysis, those which will have a greater impact on monitoring programs are the following: 1 ) developments in mass spectrometry instrumentation, such as pulsed positive-negative chemical ionization and detection techniques; 2) developments in selective detectors for gas chromatography and liquid chromatography, such as atomic absorption (AA) and atomic fluorescence (AF) spectrometry, and Fourier transform-infrared
(FT-IR) detectors; and 3) developments in computer system software capable of handling massive volumes of chromatographic as well as mass spectrometric data of the type obtained in a monitoring program. Developments in other methods such as electrochemical techniques and plasma chromatography show some promise for trace organic monitoring, but will only be useful if they can be coupled to GC or LC systems.

\section{ACKNOWLEDGEMENTS}

The technical assistance and cooperation of 1 . R. DeLeon and the help of D. Trembley in preparing this manuscript is gratefully acknowledged. The author also wishes to thank the personnel of
FDA and EPA and Mr. R. Finnigan, of Finnigan Corpora tion, for their helpful technical discussions.

\section{SELECTED REFERENCES}

1. I. H. Ford, C. A. MeDaniel, F. C. White, R. . Vest and R. F. Roberts. I. Chromatogr. Sci.. 13. 291 (1975).

2. "Principles for Fvaluating (hemicals in the Environment." National Academy of Sriences, Washington. D.C., p. $56(1975)$.

3. H. S. Hertz, W. F. May, S. A. Wise, and S.H. Chesler. Anal. Chem. 50. 429A (1978).

4. "Trace (Organic: Analysis." Environmental Science and Technology. 12, 757.(1978).

5. I. I. Laseter, I. R. Deleon, and P. C. Remele. Anal Chem. 50.1169(1978).

6. W. Mafadden. "Techniques of Combined Gas Chromatography Mass Spectrometry," Wiley-Interscience. New York, N.Y.. p. $4\{1973\}$.

7. 1). J. David. "Gas Chromatographic Detectors." Wilev Interscience. New York. N.Y. (1974).

8. P. R. Brown, "High Pressure Liquid Chromatography. Biochemical and Biomedical Applications." Academic Press. New York. N.Y. (1973).

9. C. Fenselau. Anal. Chem.. 49. 563A (1977).

10. M.L. Gross (ed.). "High Performance Mass SpecIrometry: Chemical Applications." American Chemical Sorietv. Washington. D).C.(1978).

11. I'. Cairns, Assoc. of Food/Drug Offic. Quart. Bull. 4'2, 3( 1978)

12. W. II. MoFadden, H.l. Schwartz, and S.Fvans. J. Chromatogr. 122, 389(1976)

13. P. J. Arpino. B.J. Dawkins, and F.W. McLaffertv, I (hromotogr. Sei. 12.

14. 1). I. (arroll. I. I)zidic: R. N Stillwell. K. I). Haegle. and F. C. Horning, Anal Chem. 47, 2369(1975).
15. U.S. EnvironmentalProtection Agency. "Sampling and A nalys is Proceduresfor $\mathrm{S}$ creening Indust $\mathrm{rial}$ Effluents for Priority Pollutants." ( Jincinnati. Ohio (Revised, April 1977).

16. 1. R. Politzer, S. Git hens, B. J, Dowtv and J. I. Iaraseter.J.Chromatogr.Sci.13.378 (1 975).

17. B. J. Dowty, L. E. Green, and J. L. Laseter, Anal. Chem., 48, 947( 1976]

18. I. R. Politzer, B. J. Dowty. and J. I. Iaseter, Clinical (hem. 22, 1775( 1976).

19. I). W. Kuehl. Anal. Chem.,50.182 (1978).

20. "Environmental Monitoring." VolumelV. Nat ional Academy of Sciences, Washington. ]]. (..,pp. 6-8 (1977).

21. E. I). Goldberg. V. '1'. Bowen, J. W. Farrington. (: Harvey, J. H. Martin, P. L. Pa rker. R. W. Risebrough. W. Robertson, F.Schneider, and E. Gamble, Environ. Conserv.5, 1 ( 1978).

22. ' 1 '. L. Isenhour, B. R. Kowalski, and P. C.Jurs. CKC Critical Rev in AnulChem. (July 1974).

23. E. B. Overton, (1. F. Steele, and J. L. Laseter, 'Improved Data Proressing Software fur Class Capillarv Separation of Complex Environmental Sampies, "PittsburghconferenceFet.27-Mar.3. 1978, Cleveland, ()hio. 22,

24. 1. H.Suffetand E. R. Glaser.J. Chrommtogr. Sci.16. 12 ( 1978),

25.1. W. Karasek. Industrial R'D), 20.113(April 1978).

26.E. 13. ( )verton. (; F. S I (?(?1 e. and J. 1, . Laset e r. I. H i gh Resolutonchromotography (submitted 1 978). 


\section{GLOSSARY OF ABBREVIATIONS AND TERMS USED}

\section{Abbreviations}

AA-atomic absorption

$\mathrm{AF}$-atomic fluorescence

$\mathrm{CI}$-chemical ionization

DS-data system

EC-electron capture

EI-electron impact

FID_flame ionization detector

FT-IR-Fourier transform infrared

GC — gas chromatography

GC-MS--combined gas chromatography-mass spectrometry

HPLC--high performance liquid chromatography

LC-liquid chromatography

LC-MS--combined liquid chromatography-mass spectrometry

MS-mass spectrometry

TLC--thin-layer chromatography

UV-ultraviolet

\section{Terms}

Atomic Absorption Spectrometry-a form of spectrochemical analysis usually applied to the determination of the elements. The sample is heated to a relatively high temperature to cause dissociation of the chemical compounds into atoms. A source of radiation characteristic of the element to be determined is passed through the sample. If the sample contains the element, absorption of the radiation by the sample atoms occurs and the amount of absorption can be measured for quantitative determinations.

Atomic Fluorescence Spectrometry-a form of spectrochemical analysis usually applied to many organic compounds and some inorganic compounds which emit radiation energy after they have first absorbed radiant energy of a particular frequency. The simplest form of $\mathrm{AF}$ is the fluorescence provided by a monoatomic vapor, such as sodium.

Chemical Ionization-a method for ionizing samples for mass spectrometric analysis. The ionization of the sample results from the reaction between the sample molecules and low-velocity reagent ions which results in the transfer of a charged species other than an electron.

Electron Capture Detector - a very sensitive and selective detector for gas chromatography. This detector responds to the presence of a variety of compounds containing atoms with an affinity for electrons, such as the halogens--chlorine, bromine, and fluorineand $\mathrm{o} \mathrm{t}$ her atoms, such as oxygen, and sometimes. even sulfur.

Electron Impact-a method for ionizing samples for mass spectrometric analysis. The ionization of the sample results from the bombardment under high vacuum of the sample molecules by a beam of electrons, usually at an energy of 70 electron volts $(\mathrm{eV})$.

Flame Ionization Detector-a universal detector for gas chromatography. This detector is fairly sensitive and very linear, and responds well to most organic compounds.

Fourier Transform-Infrared Spectrometry-this is the state-of-the art of infrared spectrometry. This technique employs minicomputers and Fourier transform methods in the acquisition of infrared spectra. Advantages of this technique include the making of measurements in a fraction of the time required for the more conventional methods, and increased sensitivity.

Gas Chromatography - one of the most widely used analytical techniques for trace organic analysis. The technique is simple and very rapid to use, is extremely sensitive, allowing the use of minute amounts of samples, and can be very useful for preliminary screening of environmental samples.

Gas Chromatography-Mass Spectrometry-a very powerful analytical technique that combines the features of gas chromatography and mass spectrometry.

High-Performance Liquid Chromatography-high-resolution, high-speed, and high-sensitivity liquid chromatography.

Liquid Chromatography - a separation technique that allows the partition of the sample between two phases, a liquid and a solid, or two liquid phases.

Liquid Chromatography-Mass Spectrometry-a recently developed analytical technique that combines the features of liquid chromatography and mass spectrometry.

Mass Spectrometry-a very powerful tool for providing the structural identity of complex organic molecules. Mass spectra furnish information about the arrangement of atoms within a molecule on the basis of the fragmentation pattern of the compound as a radical ion, which is usually produced by electron bombardment in the ion source of the mass spectrometry.

Thin-Layer Chromatography-a form of liquid-solid chromatography conducted on the surface of specially prepared plates. This technique is generally very quick and simple to use and is effective in performing separations of small amounts of sample.

Ultraviolet Spectrometry-a form of absorption spectrometry generally suited for analysis of compounds that are capable of absorbing ultraviolet radiation. These include aromatic compounds, conjugated ketones, and other conjugated compounds, such as polyolefins. 


\section{Analytical Systems for the Determination of Metals in Food and Water Supplies}

by R. K. Skogerboe, Ph. D.

\section{INTRODUCTION}

The analysis of food products or water supplies for toxic or potentially toxic elements, e.g., heavy metals, is the central problem addressed in this report. An evaluation of this problem in terms of the capabilities and limitations of applicable analytical techniques is the primary thrust of this report. Thus, recommendations regarding the selection of the best analytical methods and techniques for the determination of toxic elements are presented below. Before doing so, it is appropriate to delineate the criteria used in the development of the recommendations and to discuss philosophical rationales on which these judgmental guidelines are based.

\section{The Analytical Process}

Any chemical analysis can be divided into three sequential steps: 1) collection of the sample(s); 2) chemical and/or physical preparation of the sample(s) for analysis: and 3) measurement of the concentrations of the target constituents in the sample(s). Although these steps are interdependent and should not be considered otherwise, the present discussion will focus on sample preparation and measurement. Sampling should be discussed in the broader context of the overall problem of monitoring. The selection of an appropriate analytical technique must be based on the type of information desired and the purposes of collecting that information. In the present context, this may be delineated in fairly general terms.

\section{The Monitoring Question}

With rare exceptions, the central question associated with a monitoring program is:

- Are there one or more chemical entities present in the target material (e.g., food or water) in sufficient quantities to cause deleterious effects on the consumer population?

This question may be considered qualitative in that it actually requires only a yes or no answer. Given the knowledge that each of the chemicals being monitored must be present at or above some threshold' concentration before they individually or collectively produce observable effects on the recipient population, the answer is no if all are below their respective threshold effect levels and yes if one or more is above. As a result, it is quite common to use analysis approaches capable of determining or detecting the chemicals only at levels down to, but not below, their respective threshold levels. Although this practice can often be justified on an economic basis, it is subject to challenge for scientific reasons.

The astute recipient of the answer to the central question will immediately raise other questions regardless of whether the answer is positive or negative. If informed that all constituents of concern are below their individual or collective threshold levels, two questions are obvious:

- What degree of confidence can be assigned to the results?

'It must be noted that there is considerable disagreement whether or not a threshold concentration actually exists for any chemical that can deleteriously affect a recipient. Many argue, with at least some justification. that a chemical entity that affects a recipient deleteriously at any exposure concentration will cause the same effect(s) to a lesser degree even when the exposure is considerably less. While this view may prove to be totally valid, it is also certain that the effects produced must progress to certain stages before they become recognizable or measurable. In brief, the occurrence of a deleterious effect cannot be claimed unless it can be observed. The seriousness of the effect cannot be truly defined unless it can be measured. From this viewpoint, it is justifiable to define the threshold concentration as that required to produce an observable (measurable) effect on the recipient(s). This is the connotation of the term when used in this report. 
- Are the concentrations of any chemicals increasing significantly over time for a particular monitor and/or a particular collection site?

When advised that one or more constituents are present above threshold levels, these same two questions are raised and a third becomes pertinent, i.e.,

- What is the cause or source of the observed contamination?

These questions are clearly quantitative in nature. Decisions regarding possible impacts on consumer populations, or the prevention thereof, should not be based on less than quantitative and defensible information. Adherence to this philosophy is complicated by the facts that reliable designations of threshold effect levels are often lacking and that two or more contaminants may act synergistically or antagonistically.
There is one general criterion that may be defined on the basis of the above discussion. All analyses should rely on analytical methods that are capable of determining all target contaminants at concentration levels below the threshold effect levels. While methods capable of making these measurements at concentrations 100 to 1,000 times below these levels would clearly be desirable, the use of methods providing measurement capabilities 10 to 100 times below the levels may prove more practical on an economic basis.

This discussion provides a basis for delineating a general protocol for the operation of the analytical laboratory responsible for answering the above questions. A brief discussion of this is presented here to provide a general basis for succeeding topics.

\section{OPERATIONAL PROTOCOL FOR A MONITORING LABORATORY}

A general flow diagram of the laboratory operation is presented in figure $\mathrm{H}-1$. The analysis sequence given is depicted in the context of the monitoring questions, the checks required to validate the results, and the regulatory actions likely to prevail. Examination of this protocol indicates the probable need for inclusion of: 1) a quality-assurance program as a means of validating results; 2) complementary analytical methods to ensure that all required analyses can be completed and to provide comparative information relevant to validation of results; and 3) a data storage-retrieval system consistent with the requirements of the monitoring questions, the quality assurance program, and regulatory actions. The general utility of this protocol diagram and the actions it portrays will be expanded upon below.

\section{CRITERIA FOR SELECTION OF ANALYSIS METHODS}

The selection of an appropriate analysis approach must be based on the information requirements. These may be formulated on the basis of the answers to two questions:

- What are the chemical entities that must be determined?

- What are the concentration levels (threshold levels) of primary concern for these target entities?

Although answering the first question should not be particularly difficult in most instances, the issue for toxic elements has often been obscured by the use of expedient analysis approaches which may or may not provide the information required. Historically, most analysis methods used for the determination of elements simply measure the total amount of an element present without differentiating between the various chemical states of the element that may be present. Such measurements may, in fact, be relatively nonspecific indicators of potential or actual deleterious effects on biological systems. In numerous instances, the identification and measurement of the active or functioning forms of the elements is actually needed. The following examples testify to the general importance of this statement.

1. Although-arsenic is toxic, the plus three oxidation state (As(III)) is clearly more toxic than the plus five state $(\mathrm{As}(\mathrm{V}))$; the compound arsine $\left(\mathrm{AsH}_{3}\right)$ is perhaps the most toxic chemical form of arsenic.

2. Although chromium is classified as a nutritionally essential element, $\mathrm{Cr}$ (III) is toxic while $\mathrm{Cr}(\mathrm{VI})$ is relatively innocuous.

3. A measurement of the level of vitamin $B_{12}$ in animal tissue is ordinarily more useful than a 
Figure H-1. - Flow Diagram of the General Operational Protocol of a Monitoring Laboratory

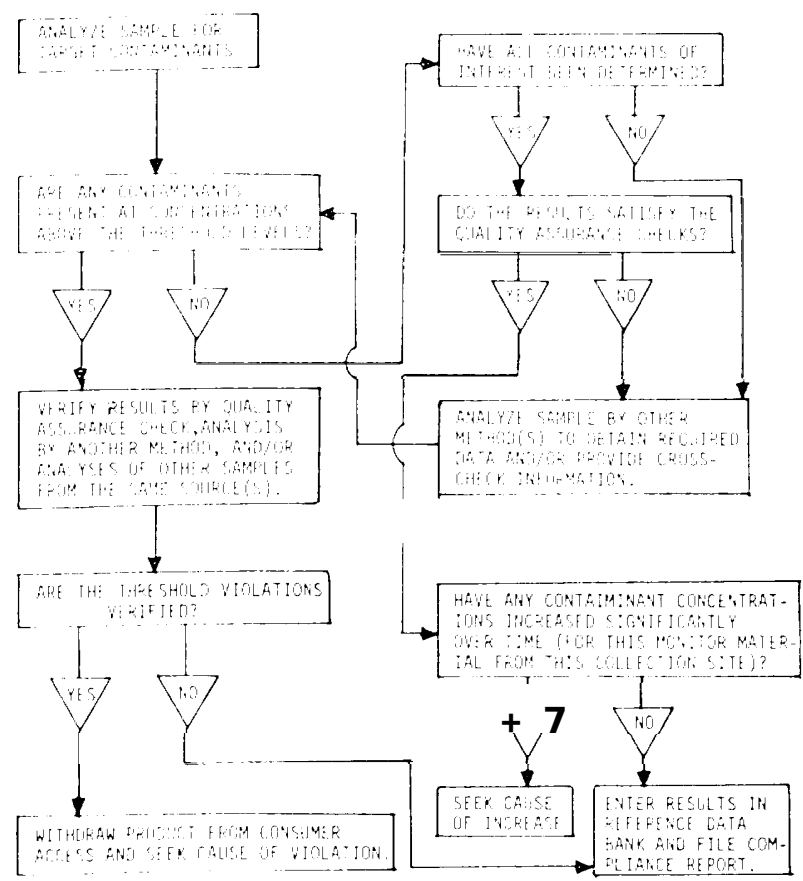

determination of tolal Co even though $\mathrm{Co}$ is the primary metal contained in $B_{1}$.

4. Alkyl (organic) mercury compounds apparently possess greater propensities for bioaccumulation and the associated health effects than the more common inorganic forms of mercury.

Clearly. chemical form or state is important! The selection of elemental analysis techniques capable of specifically measuring those rhemical forms most important from a biological effects viewpoint should be a primary objective of anv environmental monitoring program. The status of the present technology is inadequate for this purpose in many instances. As a result, the functioning forms of several elements are not well-known for various types of biological effects. Hence, the designation of the important (threshold) concentration levels for these cases has typically been rather crude. The capabilities and limitations of current analytical measurement systems are discussed in relation to the chemical form problem in the analvical instrumentation section. Research needs are also defined in that section

Although the measurement of the various chemical forms of each element is often important, it should be noted that the easier and more common measurement of the total concentration of each element (in all of its chemical forms) can be rationalized. For example, if the analysis indicates that the total concentration is below the threshold effect level for any one or combination of the particular chemical forms for an element, the need for measurement of the concentrations of the chemical forms is negated. The total concentration measurement consequently serves as a screening device indicative of the possible need for chemical form measurements.

Once the elements and the concentration ranges of interest for each have been defined, the criteria which must be invoked in the selection of an appropriate analysis method include: sensitivity, selectivity, reliability, scope, sample preparation requirements, and time-cost considerations. It would be folly to select an approach incapable of providing measurements a t or below the required concentration (threshold effect) levels. Thus, selection of a sufficiently sensitive method is of paramount importance. Although it is common to discuss sensitivity in the context of the term detection limit, it is also usually impossible to obtain a sufficiently accurate concentration measurement when said concentration is barely detectable. Thus, sensitivity should be considered in association with the term determination limit, i.e., the lowest concentration at which a sufficiently reliable concentration measurement can be carried out.

The scope of an analytical approach is defined on several bases. The ability to determine reliably large numbers of individual chemical constituents each of which may be present in a variety of sample types within a broad concentration range is the primary connotation, Thus, universal applicability (utility) is an alternate terminology implying the ideal case. It can also be argued that an analytical method which requires simple sample preparation operations offers greater scope than one requiring more complex preparative steps: the ideal case involves direct analysis of the samples without prior chemical treatment.

Analytical selectivity is usually interpreted synonymously with specificity. The analysis approach used must provide an unequivocal means of identifying each chemical constituent of interest irrespective of the compositional characteristics of the sample material being analyzed. In addition, the measurement of the concentration of a $\mathrm{n} \mathrm{y}$ constituent of interest should provide accurate results independent of the variations in the concentrations of other constituents present in the sample materials. Analysis methods that do not satisfy these conditions lack selectivity (or 
specificity): such methods are subject to what are often termed interference or matrix effects, In essence, reliability (accuracy) is the criterion which defines a sufficiently selective analysis; confidence in the results and the decision(s) based on them is explicit.

Minimization of the time-cost commitments must be an objective considered in the selection of an analysis approach. It is unfortunate that this consideration often leads to one of two extreme stances. Management often opts for the adoption of analytical technology of limited utility on the basis of lower initial capital costs and/or the fact that such technologies frequently can be applied by analysts with lower levels of expertise. Such selections are often economic errors when considered on a longer term basis, At the other extreme, management may invest in expensive facilities and expertise and then require that they be used to carry out analyses that can be accomplished more economically via another approach. This, too, can prove to be a false economy.

\section{Summary}

This background discussion has been presented as a preface to the evaluations presented below; the intent has been to provide a common basis for comments, evaluations, and suggestions which follow.

\section{PREMISES FOR SELECTION OF LABORATORY FACILITIES}

In considering the operation of a laboratory dedicated to monitoring food and water supplies for toxic elements, several premises may be established. These are essential in defining the necessary facilities and require discussion. The following list is not necessarily all inclusive nor is it set down in order of importance.

1. The majority of analysis requests will require the determination of several elements per sample.

2. To be most effective, the laboratory must be able to comply with these requests in a reasonable (short) time.

3. The analyses carried out should be accomplished at reasonable costs.

4. The analytical results must be sufficiently accurate to avoid challenge of the integrity of any decisions based on them.

The combination of these premises clearly implies that the ideal laboratory facility would be one capable of accurately analyzing for all constituents requested at concentration levels down to and below their respective threshold effect levels in a short period of time. The use of a system capable of simultaneously measuring all constituents of interest in each sample is definitely implied. The time and cost premises further imply the desirability of utilizing analysis techniques which do not require extensive sample preparation operations: the ability to directly analyze samples in an "as received" form may be considered ideal. Sample preparation operations are also primary sources of contamination or loss of the analytical constituents. The direct analysis, minimal preparations, capability is desirable from the accuracy standpoint as well. Finally, the accuracy requirement indicates the need for a highly specific analysis approach which is not subject to significant interference problems and the maintenance of a quality assurance program. No single analytical technique will necessarily satisfy all of these requirements for the elements and/or sample types of interest; a combination of techniques will be required. Properly selected, the techniques used may be complementary in terms of providing the range of elemental analyses required and in terms of providing redundant analyses for some elements. The latter will be useful for accuracy validation purposes (see discussion below).

In effect, these premises lead to the defensible conclusions that: 1) the laboratory facilities should be primarily comprised of multicomponent analysis systems; 2) more than one such system will likely be required: 3) the selection of systems that will provide some redundant analytical information is desirable; and 4) the inclusion of secondary analytical systems to be used in supportive capacities may be essential. The following evaluation is predicated on these bases. 


\section{EVALUATION OF ANALYTICAL TECHNIQUES FOR THE DETERMINATION OF TOXIC ELEMENTS}

The qualitative identification and quantitative determination of toxic or potentially toxic elements in food products and water can be based on several different analytical techniques for which commercially available instrumentation exists. The primary techniques to be considered include:

1. neutron activation analysis (NAA);

2. molecular absorption and fluorescence spectrophotometry:

3. solids (spark source) mass spectrometry (SMS);

4. atomic absorption (AAS) and atomic emission spectrophotometry (AES);

5. electrochemical techniques - anodic stripping voltammetry (ASV), - differential pulse polarography (DPP);

6. plasma emission spectrometry (PES);

7. X-ray techniques

-X-ray emission spectrometry (XES),

- proton-induced X-ray emission spectrometry (PIXE).

Each of these are considered below.

\section{Neutron Activation Analysis}

This technique is perhaps the most sensitive of those available when all elements are considered. Absolute determination limits, expressed as nanograms $\left(1 \times 10^{-9} \mathrm{gm}\right)$ that must be present in the sample for quantitative determination, are summarized in table H-1. Such determination limits should be converted to actual concentrations to lend them perspective for the present evaluation. For the analysis of food products or water, a 10gm or $10-\mathrm{ml}$ sample generally represents a reasonable upper limit on the sample size that can be activated for analysis. Thus, taking $0.5 \mathrm{ng}$ as the determination limit for arsenic as an example (table H-1) this element can be determined at approximately $0.5 \mathrm{ng} / 10 \mathrm{gm}=0,05 \mathrm{ng} / \mathrm{gm}$ ( rig/ml for water) or 0.05 parts per billion by NAA. Evaluation of the other elements of interest on this proximate basis indicates that most could readily be determined at required concentration levels via this technique. While this is an encouraging conclusion, there are other factors which detract from it.

\section{Table H-1 .-Determination Limits for Neutron Activation Analyses ${ }^{a}$}

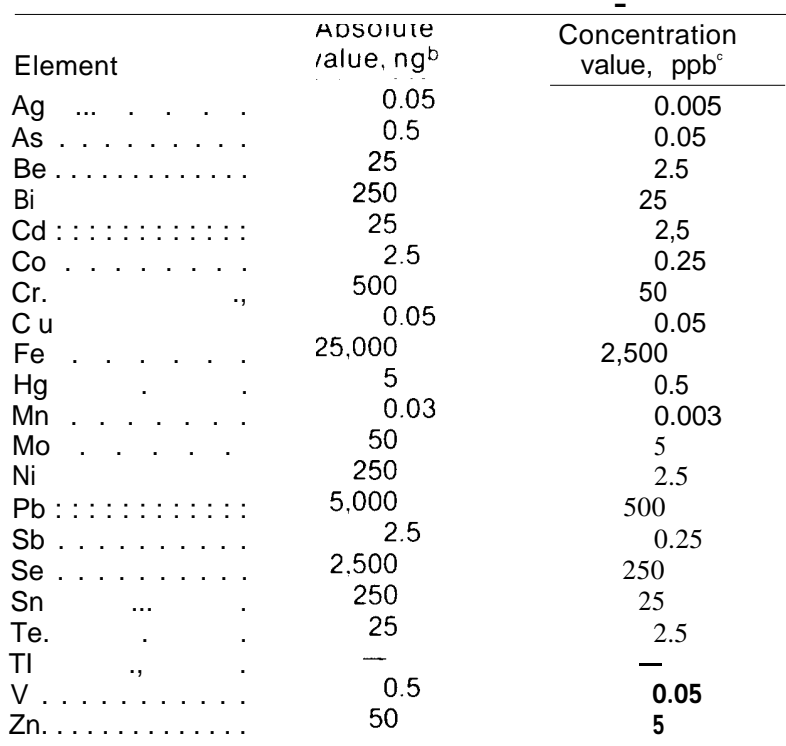

aDatataken from $\mathrm{A}$ k Skogerboe and G H Morrison, Trace Analysis E ssen tial Aspects, in Treatise on AnalyticalChemistry $(1 \mathrm{M}$ Kolthoff and $\mathrm{P} \mathrm{J} \mathrm{EI}$ ving. eds ). New York Wiley and Sons, 1971, pp 5842-5843

b Nanograms of element that must be present insample activated to Permit a quantitative determination; detection limits are approximately a factor of 5 lower Interference free measurement conditions are assumed

${ }^{c}$ Assuming $10 \mathrm{gm} \mathrm{s}_{\mathrm{amp}}$ le is act ivated, thus concen trat Ion values absolute values - 10 These values should be Increased by a factor of 100 to 1000 to compensate for the loss of neutron fluxif a neutron accelerator were used in stear of a nuclear reactor

To achieve the sensitivity required, the use of a nuclear reactor providing a high flux of thermal neutrons is required. The acquisition cost of the reactor is several million dollars; the operational costs are also comparatively high. Considerable reduction of these can be achieved by replacement of the reactor with a neutron accelerator. The neutron fluxes available with such accelerators are, however, about a factor of 100 to 1,000 less than those typical of a reactor. The analytical sensitivity available is reduced in proportion [see footnote to table $\mathrm{H}-1$ ).

If chemical separations are carried out after activation of the samples, proponents of NAA argue that analytical interferences can be virtually eliminated. This argument is often specious simply because the use of chemical separations is 
often a primary source of errors. Since such separations, even when free of errors, are time consuming and add to the expense; activation analysts usually prefer what is called the purely instrumental approach.

Two types of interferences are common to this approach. Direct interferences occur when two or more sample constituents emit radiation (gamma rays or beta particles) of nearly the same energy. If the emitting species undergo radioactive decay at significantly different rates, correction for these interferences can be based on measurement of the radiation at different times. Otherwise, the interfering species must be chemically separated prior to the measurement step. Indirect interferences due to contributions to the sample spectrum from Bremsstrahlung and Compton interactions are also common, Correction for these must be obtained by subtraction techniques. The net effect is, however, a reduction in the analytical sensitivity and/or an increase in the uncertainties associated with the measurements, While these instrumental approaches are widely used in NAA to avoid the need for chemical separations, such avoidance still restricts the potential scope of the analyses. They also tend to lengthen the time required to obtain analysis results. On these bases, NAA does not appear to be the best choice for a laboratory facility: capital costs, operational costs, and operator training requirements are primary weighting factors influencing this negative judgment.

\section{Molecular Absorption and Fluorescence Spectrophotometry}

These techniques have been used extensively for elemental analysis. They generally rely on carrying out a reaction of the element of interest with a reagent (or series of reagents) to form a product which has properties required for the absorption of light. Identification of the element incorporated into the light-absorbing product is based on the wavelength of light absorbed while measurement of the concentration relies on the extent of absorption; hence the term molecular absorption, Some light-absorbing species regain a more stable energy configuration by release of the light energy absorbed as light (fluorescence or phosphorescence). Measurement of the wavelength at which this occurs and the intensity of the light emitted is used to identify the species responsible and the amount present,

The use of these techniques requires that specific or semispecific chemical reactions be used for the formation of the absorptive and/or fluorescent products, While such reactions are generally available for the elements of interest, the analysis of any one sample for several constituents would necessarily have to rely on carrying out several individual reactions. Even then, there are only limited instances for which a reaction will occur for only one constituent (a specific reaction). Most reactions involving a particular reagent set tend to occur for each of several elements having similar properties and, as a result, their absorption of fluorescence spectra tend to be quite similar. Such spectra are subject to some degree of wavelength coincidence such that spectral interferences are not uncommon; the measurements are less specific than desirable, While other features of these techniques could be discussed, their chemical reaction requirements coupled with the specificity problem are deleterious from the multicomponent analysis standpoint. The techniques should not be classified as essential for the present elemental analysis purposes.

\section{Solids Mass Spectrometry}

This analytical technique offers sensitivity competitive with that characteristic of NAA (see table H-1) for a wide range of elements. To utilize it, nonconducting samples must be rendered electrically conducting. The constituents of an aqueous sample would ordinarily be analyzed after the water was evaporated away; the residue would be mixed with a conductor for analysis. Solid materials such as foods are also typically nonconductive; by mixing them with a conductor such as graphite or silver powder they become conductive. Food products must also be oxidized (by wet or dry oxidation techniques) to destroy the organic constituents which cause serious spectral interferences in the analysis step. ${ }^{2}$ These sample preparation steps are quite extensive and can obviously be the sources of serious errors unless carried out with caution.

In practice, the analyses must be based on two sample mixtures to obtain a complete analysis subject to less interference problems. Elemental subset A may be determined based, for example, on mixing the sample residue or ash with highpurity graphite. However, the carbon polymers of which graphite is composed are observed in the mass spectrum and preclude the possibility of analyzing for those elements that would normally

See the following" reference for a more complete discussion of the problems and capabilities: C. A. Evans and G. H. Morrison, Anal.Chem. 40.869 (1969). 
be measured at these mass positions. The analysis for elemental subset $B$ would subsequently be based on the use of high-purity silver, gold, copper, or aluminum powder as the conducting matrix. Again, the use of any of these choices to achieve sample conductivity results in spectral interferences which necessitate analyses based on two or more subsets of elements. The conductive materials used must be high purity, i.e., $6-9 \mathrm{~s}$ or 99.9999-percent pure. This requirement limits the possibilities and affects their costs, Finally, it should be noted that solid mass spectrometric analyses require unusually long times even though it is possible to obtain analyses for -40 to 60 elements on each sample. Although the sample preparations required are time consuming, the analysis itself is also rather slow. Given samples ready for analysis, a well-organized SMS laboratory would be hard-pressed to analyze more than 5 to 10 samples per man-day. These factors, coupled with high acquisition costs $(\sim 250,000)$ and high operational and instrument maintenance costs, place solids mass spectrometry in a negative posit ion relative to other possibilities,

\section{Atomic Absorption and Emission Spectrophotometry}

Recent instrument sales figures indicate that only gas chromatography is more widely used than atomic absorption spectrophotometry. When a flame is used as the energy source required to produce the gaseous atomic populations from the sample dissolved in aqueous solution, atomic absorption offers favorable analysis capabilities for a reasonably impressive array of elements (see table H-2]. Atomic emission from the same atom populations in the flame is totally complementary and supplementary to atomic absorption. Factors which affect absorption also tend to affect emission; the ultimate sensitivity achieved with either measurement approach is limited by the ability to produce the atomic populations. As a result, it can be shown that atomic absorption is generally most favorable (on the basis of sensitivity) for the determination of those elements requiring more energy to produce atomic emission, i.e., an excitation potential above approximately 4.5 electron volts $(\mathrm{eV})$. Elements with lower excitation poten-

table H-2.-Determination Limits for Flame Atomic Absorption and Emission and Furnace Atomic Absorption'

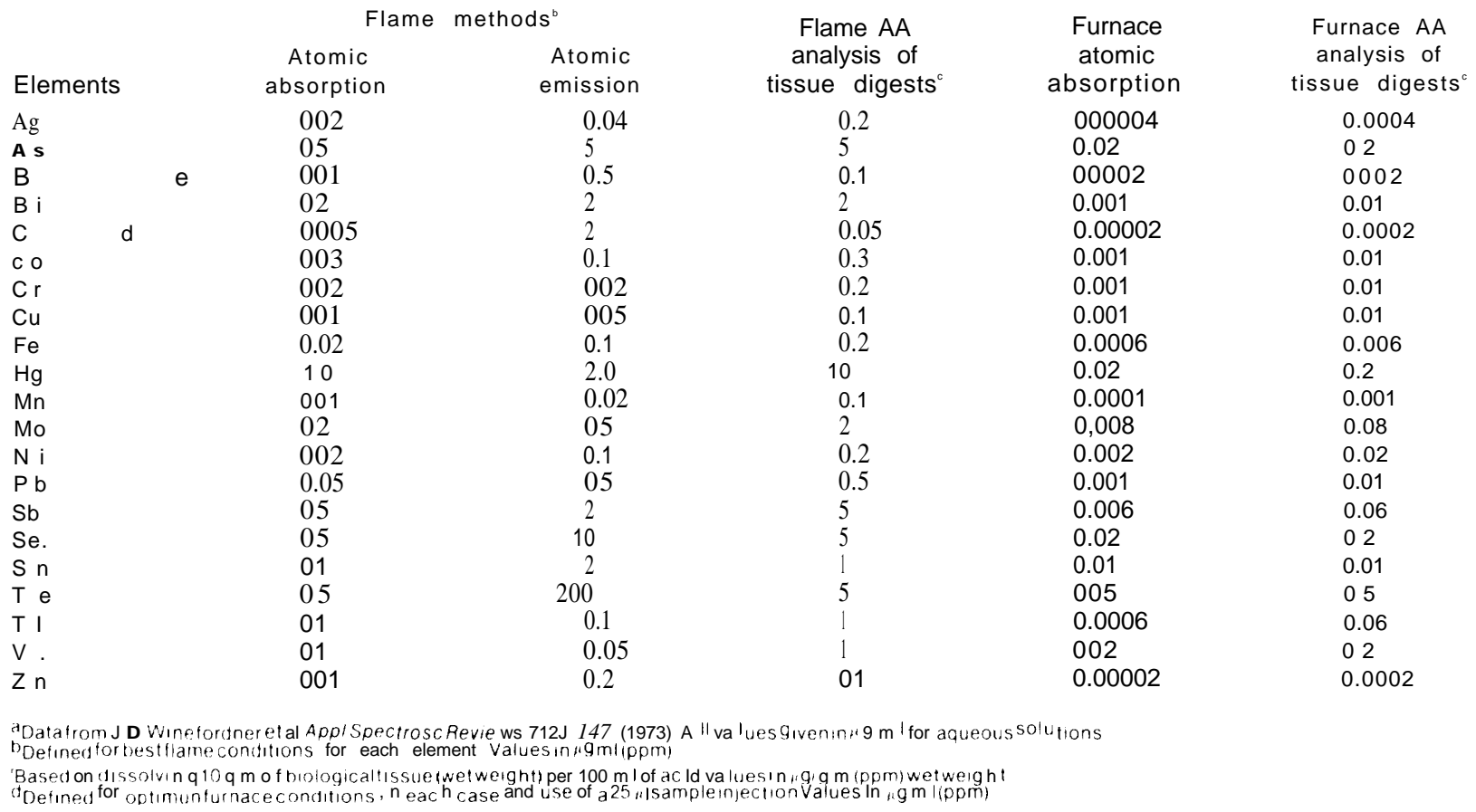


tials are best determined by atomic emission when flames are the energy media. These generalizations require the use of an instrument that is well-designed for both absorption and emission measurements (two of the three major U.S. atomic absorption manufacturers supply such instruments).

In spite of the combined capabilities offered by flame emission and absorption, they are frequently inadequate for the types of analyses in question unless preconcentration and/or separation procedures (e.g., solvent extraction) are used. These inadequacies may be due to a lack of sufficient sensitivity and/or to the occurrence of interference effects. These, coupled with the difficulties attendant to separation/preconcentration procedures, have led to the development of what is often called nonflame atomic absorption. The first significant development in this area involved the reduction of mercury ion in aqueous solution to atomic mercury $(\mathrm{Hg})$ such that it could be carried directly to the gas phase into the optical path of an AA instrument for measurement. The measurement actually involves separation of the $\mathrm{Hg}$ from the sample under conditions less subject to interferences. If carried out under appropriate conditions so that the $\mathrm{Hg}$ arrives at the measurement cell rapidly, an effective preconcentration is also realized. In effect, the measurement sensitivity is determined by the concentration of atoms delivered to the measurement cell per unit time. The nonflame methods are all designed to take advantage of this thereby enhancing the ability to analyze at lower concentrations; methodological developments for this purpose have taken two general tacts, i.e., chemical generation and furnace vaporization.

Beyond the $\mathrm{Hg}$ method described above, the hydride generation method for the determination of arsenic, selenium, germanium, lead, tellurium, tin, antimony, and bismuth has received wide attention. The hydrides of these elements are rapidly formed by reaction in acid media with sodium borohydride. The metal hydrides, being gases at ambient temperatures, are readily transported via carrier gas to a flame or a heated $\left(-800^{\circ} \mathrm{C}\right)$ quartz cell for atomization and measurement. The success of this general approach has led to the commercial availability of hydride generator accessories for atomic absorption. It has also been adopted in commercial autoanalyzer systems. Detection limits of less than $1 \mathrm{ng}$ metal/ml (1 ppb) solution are readily obtained.

Furnaces, fabricated from graphite or tungsten, that can be temperature ramped by resist- ance heating have been developed to high levels of refinement. Liquid samples are delivered to a furnace, which is resident in the optical path of an AA unit, and a programed heating cycle is initiated, In this cycle, the liquid is first evaporated at $\sim 120^{\circ} \mathrm{C}$; the salt residue remaining is "ashed" to convert it to a "common" chemical form at -3000 to $500^{\circ} \mathrm{C}$; and the ash is rapidly vaporized and atomized at $-2,0000 \mathrm{C}$ for the absorption measurement. The use of such systems provides improvements in the analytical sensitivity for most elements (see table $\mathrm{H}-2$ ). The extent of improvement is limited by the amount of sample that can be placed in the furnace $(\sim 25$ to $50 \mu 1)$ but generally amounts to a factor of 10 to 100 when compared with flame AA capabilities.

These capabilities combined with the general simplicity of operation and lower instrument costs have been largely responsible for the widespread acceptance of atomic absorption. During the principal time of AA development, the primary competing techniques were flame, arc, and sparkemission spectrometry. The last two were rather quickly abandoned by the analytical spectrometry community because they were "notably subject to interference problems associated with the vaporization-atomization system. It is rather ironic that the same community, in less than 10 years, reverted to the use of furnace AAS systems which are subject to the same interference problems for the same reasons. It is also ironic that an extensive fraction of the furnace AA research reported in the past 5 to 10 years has dealt with the study of interference effects and means for their elimination. A large percentage of these studies are reaching the same conclusions and developing the same compensatory methods that resulted from arc and spark spectrometry interference effect studies before the advent of atomic absorption. Nevertheless, atomic absorption analysis is wellestablished and here to stay. The furnace methods, in particular, clearly offer the required sensitivities for a wide range of analysis problems.

A primary historic limitation has been that atomic absorption has been basically a single element analysis technique; analyses for several elements in a sample are carried out sequentially in time. The emergence of plasma emission spectrometric systems which allow the simultaneous analysis of several elements (to be discussed below) has forced the atomic absorption community to the development of multicomponent analysis systems. Only one commercial instrument offering this capability for more than two elements is available at this date, This unit is, in fact, a se- 
quential analysis system since the elements of interest are not measured simultaneously. Its use saves analysis time but not as much as it would in the simultaneous mode. At least one other manufacturer will introduce a truly simultaneous multielement AA analysis system in the next 1 to 2 years. Prototypes of such a unit have been developed and tested at Colorado State University. ${ }^{3}$ The tests have shown that sets of 5 to 10 elements can be determined simultaneously without sacrifice of analytical sensitivity. The availability of such instrumentation will advance the state of the art for atomic absorption.

The inclusion of atomic absorption in an elemental analysis laboratory may be considered worthwhile as a secondary facility at least. If said instrument offers the simultaneous measurement capability, the inclusion may be justified on a better economic basis.

\section{Electrochemical Techniques}

These techniques are perhaps the most classical of those considered herein, And yet, electrochemistry has been reborn in the past two decades largely through the development of what may be classified as pulse or differential techniques. The electrochemical techniques of primary interest in the present context rely on the measurement of the Faradaic current (FC) produced or used during oxidation or reduction reactions involving the element to be determined; the potentials at which FC changes occur depend on the elements (ions) involved in the reactions. Such reactions are almost universally accompanied by non-Faradaic processes involving other sample constituents which also result in the production or utilization of current. In classical direct current polarography or voltammetry, the ability to measure a low Faradaic current (low analyte concentration) is limited by the magnitude of the nonFaradaic current changes occurring simultaneously. The revitalization of electrochemistry has been largely based on the fact that when the potential required to induce a redox reaction is removed, the non-Faradaic current decays more rapidly than the Faradaic current. Thus, by "pulsing" the potential up to the reaction level required, shifting it back to below the reaction level, and waiting an appropriate time period for the non-Faradaic current to decay; the Faradaic to non-Faradaic current ratio can be significantly

'See for example: F. S. Chuang, D. F. S. Natusch, and K. R. O'Keefe, Anal, Chem. 50, 525 [1978). improved. A variety of these differential pulse approaches have been developed to achieve analytical measurements at concentrations 10 to 1,000 times lower than previously possible. The techniques which appear to offer the most significant capabilities are differential pulse polarography (DPP) and differential pulse anodic stripping voltammetry (DPASV). At least one commercial instrument offering both of these measurement capabilities is available.

The electrochemical literature indicates that -16 to 20 elements can be determined by DPP, DPASV, or cathodic stripping voltammetry (CSV). This is true, but all such elements cannot be determined under a single set of experimental conditions. Some must be determined using a gold working electrode (for example) while others require the use of a mercury electrode. Some determinations require the use of a specific supporting electrolyte solution while others do not. Finally, the redox potentials of some entities are sufficiently similar that they cannot be determined in the presence of each other without the use of correction techniques. In some cases, prior separations are required.

The electrochemical analysis literature indicates that $\mathrm{Cd}, \mathrm{Cu}, \mathrm{Pb}, \mathrm{Zn}, \mathrm{Tl}$, and $\mathrm{Fe}$ can usually be determined simultaneously in a single supporting electrolyte solution using mercury as the working electrode material, These elements can be determined at levels of $1 \mathrm{ng} / \mathrm{ml}$ or less in the electrolyte solution by DPP or DPASV. As a result, these techniques are quite widely used for the analysis of the above elements. Although $\mathrm{Cu}$ and $\mathrm{Zn}$ interfere with each other via formation of the $\mathrm{Cu}: \mathrm{Zn}$ intermetallic in the mercury electrode and the reduction of $\mathrm{Fe}$ (III) to $\mathrm{Fe}$ (II) interferes with the $\mathrm{Cu}$ determination, these can be corrected via use of expedient instrumental procedures. Other elements frequently determined are As(III) by DPASV or DPP and $\mathrm{Se}(\mathrm{H})$ by CSV. Again, analyses at or below the part per billion concentration level are common.

The electrochemical methods detect only the electroactive species, e.g., the ions, This statement must be qualified in terms of the time-scale of the measurement step. To illustrate, consider an electroactive metal ion $\left(\mathrm{M}^{+}\right)$which may be present in the sample solution primarily as a complexed or molecular species designated, for example, by $\mathrm{MX}_{\mathrm{n}}$. To measure the total $\mathrm{M}^{+\mathrm{n}}$ plus $\mathrm{MX}_{\mathrm{n}}$ concentration, the following reaction must occur either prior to or during the measurement period:

$$
\mathrm{MX}_{\mathrm{n}} \rightarrow \mathrm{M}+\mathrm{n}+\mathrm{nX}-
$$

If the duration of the measurement is short, the above reaction must go to a reproducible state of 
completion within that time period; in such cases the complexed/molecular entity may be classed as labile. Thus, there is a growing investigative effort in the electrochemical community involving the use of this conceptual approach as a means for the identification of the chemical forms of metals in natural systems. The general thrust of these efforts involves either the use of chemical or electrochemical means for systematically shifting the above type of reactions toward the formation of an electroactive species for identification purposes. A typical electrochemically induced shift experiment, for example, might involve ASV. By electrodepositing from the sample solution for successively longer periods and measuring the amount of $\mathrm{M}+$ " reduced during each period, the time required to drive the above type of reaction to completion might be deduced. This may be indicative of the "lability" of the complexed/molecular form, i.e., its thermodynamic or kinetic stability, Extensive research must be completed to establish the potential utility of such approaches. However, these possibilities combined with the high sensitivities that can be achieved with modern electrochemical techniques suggest that such facilities should prove to be valuable laboratory facilities. (See further discussion below.)

\section{Plasma Emission Spectrometry}

It has been suggested that atomic emission spectrometry offers what may approximate the ideal approach to multielement analyses. Indeed, the use of the radio frequency inductively coupled plasma (ICP) as the atomic excitation source in combination with a direct-reading emission spectrometer permits the simultaneous determination of numerous elements at low concentration levels. ${ }^{4}$ Similarly, the use of a direct current plasma (DCP) excitation source for direct-reading emission spectrometry shows comparable promise. The evolution of such systems over the past decade has brought emission spectrometric analysis back to the forefront of analytical capabilities. Such plasmas are principally used to excite analytical constituents delivered to them via solution nebulization (aerosol production) systems. The conversion of solid samples to aqueous solutions for analysis (e.g., by wet oxidation) results in elimination of many of the major compositional differences between samples such that interfer-

4 good introductory review has been presented by V.A. Fassel and R. N. Kniseley. Anal. Chem, 46, 11 10A (1974). See also, Science, 202, 183( 1978). ence effects due to matrix differences may be eliminated or reduced. As a result, a single set of operational conditions may be used for the simultaneous determination of 20 to 60 elements,

Quantitative determination limits for the ICPand DCP-Multielement Atomic Emission Analysis Systems (MAES) are listed in table H-3. Examination of these data indicates that these systems are adequate for the simultaneous determination of a major fraction of the elements of interest at levels commensurate with the anticipated threshold effect concentrations. This is one reason why the ICP-MAES and DCP-MAES manufacturers have enjoyed significant annual sales improvements over the past 5 years.

Some elements of high concern, because of their toxicities or propensities for bioaccumulation, cannot be determined at low enough concentrations by direct solution analysis, e.g., $\mathrm{Hg}$, As, and Se. However, the hydride generation approaches used to solve this problem when AAS is the analysis method, are equally applicable to MAES. In fact, all hydride-forming elements can

\section{Table H-3.-Multielement Atomic Emission Determination Limits for Two Common Plasma Excitation Systems ${ }^{\mathrm{a}}$}

\begin{tabular}{|c|c|c|c|c|}
\hline \multirow[b]{2}{*}{ Element } & \multicolumn{2}{|c|}{ ICP-MAES } & \multicolumn{2}{|c|}{ DCP-MAES } \\
\hline & $\begin{array}{c}\text { In solution, } \\
\mu \mathrm{g} / \mathrm{ml}\end{array}$ & $\begin{array}{l}\text { For tissue } \\
\text { digest in } \\
\mu \mathrm{g} / \mathrm{gm}(\mathrm{ppm})^{\mathrm{b}}\end{array}$ & $\begin{array}{l}\text { solution, } \\
\mu \mathrm{g} / \mathrm{ml}\end{array}$ & $\begin{array}{c}\text { Tissue } \\
\text { digest } \\
\text { g/gin }(p p m)^{b}\end{array}$ \\
\hline $\mathrm{Ag} \ldots .$. & 0.01 & 0.1 & - & - \\
\hline As $\ldots$. & 0.3 & 3 & 0.2 & 2 \\
\hline$B \ldots \ldots$ & 0.02 & 0.2 & 0.02 & 0.2 \\
\hline $\mathrm{Be} \ldots \ldots$ & 0.002 & 0.02 & 0.05 & 0.5 \\
\hline $\mathrm{Bi} \ldots \ldots$ & 0.2 & 2 & - & - \\
\hline Cd ..... & 0.01 & 0.1 & 0.05 & 0.5 \\
\hline Co ...... & 0.01 & 0.1 & 0.08 & 0.8 \\
\hline $\mathrm{Cr} \ldots .$. & 0.02 & 0.2 & 0.02 & 0.2 \\
\hline $\mathrm{Cu} . . .$. & 0.005 & 0.05 & 0.01 & 0.1 \\
\hline $\mathrm{Fe} \ldots$. & 0,01 & 01 & 0.01 & 0.1 \\
\hline $\mathrm{Hg} \ldots .$. & 0.1 & 1 & 0.01 & 0.1 \\
\hline $\mathrm{Mn} \ldots$. & 001 & 01 & 0.05 & 0.5 \\
\hline Mo..... & 0.03 & 0.3 & 0.05 & 0.5 \\
\hline $\mathrm{Ni} \ldots .$. & 0.05 & 0.5 & 0.05 & 0.5 \\
\hline $\mathrm{Pb} \ldots$. & 0.1 & 1 & 0.05 & 0.5 \\
\hline $\mathrm{Sb} \ldots .$. & 1 & 10 & - & - \\
\hline $\mathrm{Se} \ldots .$. & 0.2 & 2 & 0.2 & 2.0 \\
\hline Sn & 1 & 10 & 1 & 10 \\
\hline $\mathrm{Te} \ldots .$. & 0.4 & 4 & - & - \\
\hline TI ..... & 1 & 10 & - & - \\
\hline $\mathrm{U} \ldots$. & 0,2 & 2 & 0.5 & 5 \\
\hline$\vee \ldots \ldots$ & 0,02 & 0.2 & 0.02 & 0.2 \\
\hline $\mathrm{Zn} \ldots$. & 0.01 & 0.1 & 0.02 & 0,2 \\
\hline
\end{tabular}

aDatafor the ICP from R K Winge et al Spectrochim Acta, $32 B 327$ ( 1977) Data for the DCP from R K Skogerboe H E Taylor and G W Johnson, Spec

trochim Acta in press
ofor $10 \mathrm{gm}$ ol biological $\mathrm{issue}$ (wet wt) dissolved in $100 \mathrm{ml}$ of solution 
be determined at required concentrations on a simultaneous basis. (Tissue detection limits of 0.01 $\mu \mathrm{g} / \mathrm{gm}$ (ppm wet weight) can be achieved. )

Although the measurement of atomic emission offers a high degree of qualitative specificity, there are two common types of interferences which affect the quantitative specificities of such systems. Both plasma types are highly efficient excitation media. As a result, extremely intense radiation from common elements such as $\mathrm{Ca}$ and $\mathrm{Mg}$ is delivered to the spectrometer-measurement system, The result is the observation of stray light interference effects for some other elements. The magnitudes of these effects depend on: the design characteristics of the spectrometer used, the concentrations of the elements from which the stray light originates, the concentrations of the elements subject to the interferences, and the types of approaches used to alleviate the effects. An expedient means of correcting for these effects may be based on determination of the concentrations of the causative elements. To illustrate, let $\mathrm{C}_{\mathrm{m}}$ represent the measured concentration of a particular element (analyte) subject to interference due to a concomitant constituent present at concentration $C_{C}:$ The true (corrected) concentration $\left(\mathrm{C}_{\Gamma}\right)$ of the analyte may be determined from:

$$
\mathrm{C}_{\mathrm{T}}=\mathrm{C}_{\mathrm{m}}-\mathrm{aC}_{\mathrm{C}}
$$

where a is a correction coefficient determined by simple experimental procedures.

The other type of interference effect involves interelemental processes in which the presence of one constituent changes the extent of excitation of another in the plasma, While such interelement effects are less common, the above correction procedure can also be used for compensation. Commercial ICP- and DCP-MAES systems are routinely equipped with minicomputer or microprocessor systems for control, data acquisition, and data correction purposes. The use of the types of corrective procedures described above is, thus, easily automated.

In effect, the general capabilities of plasmaMAES are such that it should be considered a primary facility.

\section{X-Ray Emission Techniques}

The bombardment of samples with $\mathrm{X}$-rays to produce X-ray fluorescence (XRF) has remained in wide usage. In fact, the development of the lithium drifted germanium, $\mathrm{Ge}(\mathrm{Li})$, or silicon, $\mathrm{Si}(\mathrm{Li})$, detectors has advanced the status of this approach by the reduction of spectral interference problems and a general increase in the sensitivity available. Such energy-dispersive detection systems have rather rapidly replaced the conventional wavelength-dispersion units. The production of $\mathrm{X}$-ray fluorescence (emission) by X-ray bombardment and measurement with an energy dispersive detector offers reasonable analysis capabilities for several elements of interest (see table H-4).

In 1970 , the potential of heavy, charged particles for X-ray excitation was recognized, and improved capabilities have been demonstrated. The capability improvements of accelerator (particle) beams are due to: 1) the high particle fluxes available, z) the relatively low background radiation associated with the excitation process: 3 ) the fact that the excitation cross-sections of many elements for particles are higher than for photons or electrons, and 4) a single charged particle can induce emission of several X-ray photons as it pene-

\section{Table H-4. - Determination Limits for X-Ray} Fluorescence Techniques

\begin{tabular}{|c|c|c|c|c|}
\hline \multirow[b]{2}{*}{ Element } & \multicolumn{2}{|c|}{ X-ray flourescencea } & $\begin{array}{l}\text { Proto } \\
\text { X-ray }\end{array}$ & $\begin{array}{l}n \text { induced } \\
\text { emission }\end{array}$ \\
\hline & $\mathrm{ng} / \mathrm{cm}^{2 \mathrm{c}}$ & $\begin{array}{c}0.1 \mathrm{cc} d r y \\
\text { tissue, } \mu \mathrm{g} / \mathrm{gm}^{d}\end{array}$ & $\mathrm{ng} / \mathrm{Cm}^{2 \mathrm{~d}}$ & $\begin{array}{c}0.1 \mathrm{cc} d r y \\
\text { tissue, } \mu \mathrm{g} / \mathrm{gm}^{d}\end{array}$ \\
\hline$\ldots$ & -e & $-e$ & 50 & 0.3 \\
\hline Al...... & 800 & 4 & $-e$ & - $^{e}$ \\
\hline As $\ldots \ldots$. & 8 & 0.04 & 5 & 0.03 \\
\hline $\mathrm{Ba} \ldots$. & 120 & 0.6 & - & - \\
\hline $\mathrm{Bi} \ldots \ldots$ & - & - & 20 & 0.1 \\
\hline $\mathrm{Cd} \ldots . .$. & 20 & 0.1 & 50 & 0.3 \\
\hline Co ..... & - & - & 3 & 0.02 \\
\hline $\mathrm{Cr} \ldots \ldots$ & - & - & 3 & 0.02 \\
\hline $\mathrm{Cu} \ldots .$. & - & - & 4 & 0.02 \\
\hline $\mathrm{Fe} \ldots \ldots$ & 25 & 0.1 & 3 & 0.02 \\
\hline $\mathrm{Hg} \ldots .$. & - & - & 20 & 0.1 \\
\hline$M n \ldots$. & - & - & 3 & 0.02 \\
\hline Mo...... & - & - & 30 & 0.2 \\
\hline $\mathrm{Ni} \ldots . .$. & - & - & 4 & 0.02 \\
\hline $\mathrm{Pb} \ldots$. & 25 & 0.1 & 20 & 0.1 \\
\hline $\mathrm{Sb} \ldots$. & - & - & 150 & 0.8 \\
\hline $\mathrm{Se} \ldots .$. & 10 & 0.05 & 5 & 0.03 \\
\hline $\mathrm{Sn} \ldots .$. & 25 & 0.1 & 150 & 0.8 \\
\hline Sr...... & 10 & 0.05 & - & - \\
\hline $\mathrm{Te} \ldots .$. & - & - & 150 & 0.8 \\
\hline TI ...... & - & - & 20 & 0.1 \\
\hline$V \ldots \ldots$ & 50 & 0.3 & 3 & 0.02 \\
\hline$Z n \ldots$ & 15 & 0.08 & 4 & 0.02 \\
\hline
\end{tabular}

aData from J.M Jaklevic and $R$. L Walter in 'X-ray Flourescence Analysis of Environmental Samples" (T G. Dzubay, ed.) Ann Arbor Publishers. Inc. 1977. p 68 Excitation with $\mathrm{Cr}$ or $\mathrm{Rh}$ X-ray tubes and measurement with a $\mathrm{S}$ (ILi) energy dispersive detector

energy dispersive detector
bData from C J Umbarger et al.. "Advances in X.Ray Analysis." vol 16 (L. S. Birks et al eds I. Plenum Press. N.Y..1973. pp 102-110. 3 Mev protons used

${ }^{\mathrm{C}}$ Nanograms element per square $\mathrm{cm}$ of sample surtace could be converted to solution analysis capabilities by evaporating $X \mathrm{ml}$ of liquid on a $1 \mathrm{~cm}^{2}$ area for analysis

$d_{1} \mathrm{~cm}^{2} \times 0.1 \mathrm{~cm}$ thick dry tissue: wet weight of $0.2 \mathrm{gm}$ assumed to estimate determination limit in $/ \mathrm{g}$ gm (ppm) wet weight

eFalure to cite a value due to lack of data: does not necessarily indicate analysis is impossible 
trates the sample. As a result, proton induced $\mathrm{X}$ ray emission analysis (PIXE) has rapidly emerged as a sensitive analysis approach which may be subject to fewer interference problems than $\mathrm{X}$ ray induced emission. Thus, the $\mathrm{X}$-ray source is replaced by a van de Graaff accelerator to produce proton beams in the 2.5 to $3.0 \mathrm{Mev}$ energy range.

A potentially significant capability associated with PIXE analysis is that of direct analysis of biological tissue sections or blood. Recent publications ${ }^{56}$ have shown that $\mathrm{Cl}, \mathrm{K}, \mathrm{Fe}, \mathrm{Cu}, \mathrm{Zn}, \mathrm{Pb}, \mathrm{Se}$, $\mathrm{Br}, \mathrm{Sr}$, and $\mathrm{S}$ could be directly measured in a 30 $\mu \mathrm{m}$ thick section of human kidney; that the same elements plus $\mathrm{Mn}, \mathrm{Ni}, \mathrm{Hg}, \mathrm{Rb}$, and $\mathrm{Zr}$ could be determined in a thick section of carp muscle; and that several elements can be directly measured in tissue sections of liver, kidney, lung, and bone. The analysis of liquid- or wet-digested materials may be based on evaporation of the liquid phase to leave a residue deposit on an appropriate ana-

J. L. Campbell et al. "Advances in X-ray Analysis," vol. 17 (C. L. Grant et al.,eds.). Plenum Press, New York, 1974, pp. 457-466.

'P. S. Ong et al., [bid., vol. 16 (L. S. Birks et al., eds. ), 1973, pp. 124-133. lytical substrate, Ions in solution may also be preconcentrated for analysis using filters impregnated with ion exchange resins. 'Determination capabilities for XRF and PIXE are listed in table H-4,

The general capabilities of these techniques are such that they can be used in a laboratory of the type considered. It should be emphasized, however, that quantitation of the measurements is subject to difficulties particularly for direct measurements. Proponents of the techniques argue that these problems can be readily overcome; others (cynics??) argue that this will require extensive development efforts. The truth appears to be intermediate between these extremes. The ultimate decision to include the X-ray capabilities in the laboratory facility should probably be based on the essentiality (desirability) of being able to analyze solid samples (e.g., tissue thin sections) directly for several elements of potential interest. This capability may be considered by some to be advantageous simply from the semiquantitative screening standpoint.

S. L. Law and W. J, Campbell, "Advances in X-ray Analysis, ” vol. 17, Plenum Press, New York, 1974, pp. 457-466.

\section{PREPARATION OF SOLID MATERIALS (BIOLOGICAL TISSUE) FOR ANALYSIS}

Many techniques require that solid samples be converted to solution form for analysis. The literature on methodology for sample decomposition is immense, The procedures cited find both wet(acid digestion) and dry-(ashing) oxidation methods extensively used. Dry-ashing methods are usually implicated when problems with recovery or losses of analytical constituents are reported. In comparing wet- and dry-ashing methods, the paucity of data specific to real-life samples makes it inappropriate to state categorically that one method is superior to another. Some generalizations can, however, be made on the basis of procedural differences. Wet oxidation has the advantage of requiring a minimum of apparatus and is less prone to volatilization and retention losses than dry-ashing. Wet-ashing may suffer in that relatively large amounts of reagents having significant levels of contamination may be required and contact with glassware may account for a higher risk of contamination than dry-ashing. Dry-ashing requires few, if any, reagents and handling of larger samples presents less problems. The risk of volatilization, convection, and retention losses is higher, however, unless ashing conditions are carefully controlled, These risks have led to the fairly widespread adoption of low temperature ashing (LTA) based on reaction of the sample of the oxygen free radical generated via a radio frequency field. While it is true that materials can be converted to oxides under these conditions, it is also apparent that several elements may still be lost by volatilization at typical operational temperatures of $100^{\circ}$ to $150^{\circ} \mathrm{C}$. These are largely elements with a tendency to form volatile chlorides, oxychlorides, or hydrides, Thus, the extent of their loss may be particularly influenced by the halogen (chloride) content of each sample, The use of programed-temperature ashing furnaces has been shown to be effective in preventing or reducing volatilization losses. Raising the temperature at a rate permitting slow charring and oxidation of the sample is to be highly recommended.

Nitric acid is a universally used wet-oxidant, The azeotrope boils at $120^{\circ} \mathrm{C}$, a factor which assists in its removal after oxidation but also limits 
its effectiveness in completing the oxidation process. The most effective medium for wet oxidation is a mixture of nitric and perchloric acid; the azeotrope boils at $180^{\circ} \mathrm{C}$ to force oxidation. Extraordinary care to avoid explosions and fume hoods is required. The precaution of keeping sufficient nitric acid present until all easily oxidized material is gone (cessation of brown fumes) is particularly important in wet digestion of tissues having lipid (fat) contents. Because the acids used are never absolutely free of metal contamination, the objective of wet oxidation should always be to complete the oxidation with the smallest possible amount of acid. This minimizes the blank problem. The use of reflux digestion apparatus equipped with condensers is focused on this objective as well as that of minimizing losses. Recent systems which rely on microwave ovens for heating the acid-sample mixture also show considerable promise. ${ }^{89}$ The microwave system heats the solu-

"A. Abu-Samra, J.S. Morris, and S. R. Koirtyohann. Anal. Chem., 47. 1475 (1975].

"U. S. Patent No. 4,080.168 (Mar. 21, 1978). tion rapidly and prevents bumping and frothing. Recovery studies run on a range of elements suggest that loss problems are minimal. Bovine liver is notorious for being difficult to wet digest. The report ${ }^{10}$ that $2 \mathrm{gm}$ (wet weight) or $1 \mathrm{gm}$ (dry weight) of beef liver can be digested in $10 \mathrm{ml}$ of nitric/perchloric acid in 2 to 3 minutes indicates an attractive capability that will surely guarantee extensive use of the microwave system.

For any of the analysis techniques which require sample dissolution, the approximate upper limit of "dissolved salts" that can be tolerated is 2 percent (wt/vol). Thus, for a wet biological tissue which yields 10 percent ash, this upper limit would be equivalent to dissolving $20 \mathrm{gm}$ of the tissue (wet wt) per $100 \mathrm{ml}$ of analytical solution. Working with half that weight per $100 \mathrm{ml}$ would have practical advantages.

“'Report to the U.S. FDA (Contract No. 223-75-2268] Dec..2. 1976.

\section{ASSURANCE OF ANALYTICAL ACCURACY}

Quality assurance in an analysis laboratory refers specifically to the question: Are the analytical results valid (accurate or reliable]? Obtaining the answer is complicated by the fact that all analyses are subject to random (indeterminate) errors and may also be subject to systematic (determinate) errors. Errors are cumulative; those which characterize the accuracy of any analysis result may include the composite contributions of the random and systematic errors inherent in the analysis method(s) used and those characteristic of each analyst, Laboratory, or set of equipment involved in the analysis. To maintain quality assurance, an operating laboratory must base its program on some combination of the possible strategic approaches. These include:

1. Recycling of submitted samples to obtain cross check analyses,

2. Recycling of certified or secondary standard reference materials to estimate analytical accuracy,

3. Spike-recovery studies to estimate accuracy,

4. Participation in collaborative test programs, and

5. Comparison of results obtained by independent analysis methods.

Each of these provides specific types of relevant information but all should be included in the program. Moreover, any of the above tests should be carried out incognito. Otherwise, the analysts may be tempted to devote inordinate attention to the check analyses.

All of these methods add to the expense of lab operation. It is necessary, however, for the lab personnel to be acutely aware of the ways in which they or their analytical methods can fail and (ideally) know when they have failed. Otherwise, their products-the analysis results-will be subject to challenge. Experience with the legal process suggests that the agreement between results obtained by two or more independent analytical methods is a primary indicator of success (accurate analyses). Thus, the planned inclusion of analysis redundancy ( 5 above in particular) in the laboratory operation is to be recommended; a typical quality assurance program would involve check analyses amounting to 5 to 10 percent of the total load.

' I An expanded discussion of quality assurance programs and the necessity thereof is given in the paper by R. K. Skogerboe and S. R.Koirtyohann, "Accuracy Assurance in the Analysis of Environmental Samples.” NBS Special Bulletin 422 ( 1976). 


\section{LABORATORY INSTRUMENTATION AND FACILITIES REQUIREMENTS}

Consideration of the above discussion indicates that the majority of the analytical requirements can be satisfied by plasma emission spectrometry or by atomic absorption spectrometry with heavy reliance on furnace atomization systems. The inclusion of more than one measurement technique in the laboratory can also be justified on various bases. The advantages, limitations, and tradeoffs involved will be considered below. Regardless of which instrumentation facilities are chosen as primary; the space requirements, ancillary facilities requirements, and personnel requirements are comparable. Thus, discussion of these prior to consideration of the analytical instrumentation facilities is appropriate,

\section{Personnel, Space, and Ancillary Facility Requirements}

The general design of the laboratory should include four physically separated types of space: 1) office space for personnel, 2) a sample receiving and storage room, 3) a sample preparation laboratory, and 4) the analysis laboratory. Working desk space should be included in the last three types of space in addition to the facilities discussed below. All lab facilities will require temperature control to plus or minus $5^{\circ} \mathrm{F}$ and humidity control (less than 50 percent).

It is recommended that computer capabilities should be a primary ancillary facility included in the laboratory operation. This is based on several rationales including:

1. several types of instrumentation likely to be present are most effectively used under a computer control-data acquisition mode of operation:

2. personnel requirements, time commitments, and human errors can be reduced in a computer-oriented operation; and

3. a computer system may be essential as a data management, quality assurance evaluation, warning assessment, and report preparation tool in a laboratory operation of the size likely for the present program.

Although the computer configuration selected will be dependent on the laboratory purpose(s), the analytical facilities installed, the size of the operation, and other subsidiary factors, it is likely a computer acquired with one of the instruments described below could be adapted for use in an in- tralaboratory, interactive (time-shared) mode and tied to a larger computer system (external to the laboratory) which would perform those data management functions that need not be carried out on a real-time (fast response) basis. Systems which use internal, instrumentation-coupled, computers for control and data acquisition purposes and transmit the data to central management computers are presently in operation at the USGS Water Resources Laboratory in Denver and the EPA Laboratories in Cincinnati. Although these operations may not be the best model examples, the conceptual approach which they embody is recommended for the present operation. Further comments relating to this are inserted in appropriate sections which follow.

Sample Receiving and Storage Room-The receiving operation will necessarily include facilities for logging in samples and the associated analytical work requests. A computer terminal should provide the most effective capability. The storage facilities should include: shelving or cabinets for those samples that can be stored under ambient conditions, a cold room $\left(4^{\circ} \mathrm{C}\right)$ for storage of water samples, and a freezer for storage of certain biological samples. The size of the room and storage facilities required will depend on the anticipated sample load and variations in the sample submission rate. Since such data have not been supplied, size estimates and costs are not included in this report,

Sample Preparation Laboratory-The design of this laboratory will be sample type and sample load dependent. If the sample types to be analyzed include those which must be ground in the dry form such that atmospheric contamination can occur, the grinding facilities should be physically isolated from the other sample preparation operations, In addition, the sample preparation lab should basically be a clean room $^{12}$ operation or it should be equipped with laminar flow, filter hoods in which certain sample preparation operations can be carried out. Both wet- and dry-oxida-

\footnotetext{
Clean room or clean hood environments are classified on the basis of controlling the concentrations of particles (dust) in air whichcan rontaminate the samples by fallout. A class-100 clean room, for example, must have no more than 100 particles in the 0.5- to 5- micrometer diameter size range per cubic foot of air. A class- 1,000 room permits 1,000 pa rticulates per cubic foot in the above range. ' 1 'o meet these specifications, air filtration is required.See the 1963 Federalstandard No. 209:1,
} 
Table H-6.-Summary of Cost-Productivity Estimates for Various Analytical Techniques

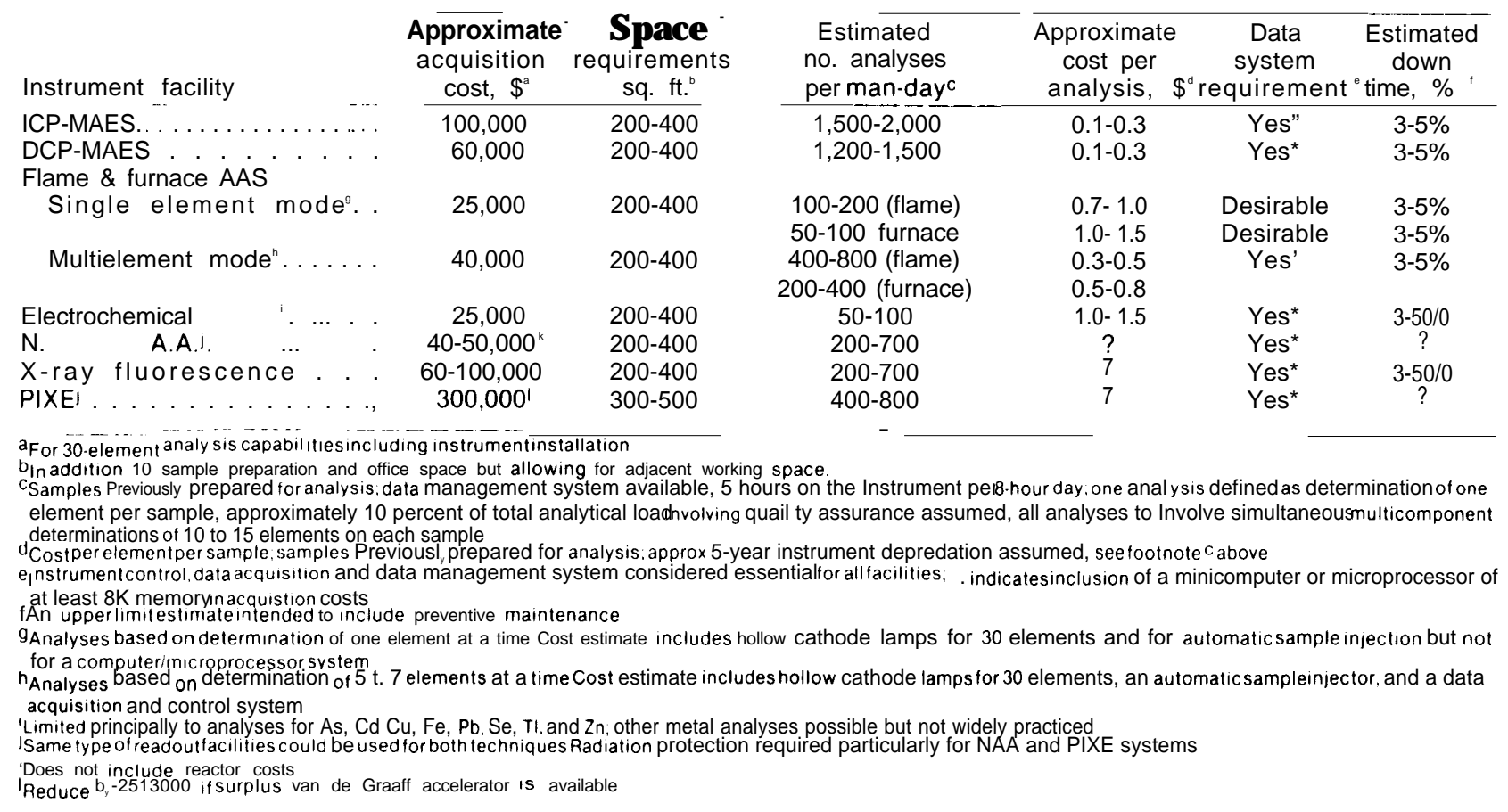

structure, to actual types and numbers of analyses to be performed, the efficiencies of the employees involved, and the final laboratory design configuration, The instrument acquisition estimates include initial installation and assume that the experience of the operating analyst will minimize startup time requirements. The estimated number of analyses per 8-hour day assume samples have been previously prepared for the analysis; that the instrument operation will rely on computer-controlled data acquisition and management; that the salary of the operation would approximate $\$ 20,000$ to $\$ 24,000$ per annum; and that the instrument would depreciate completely over a 5-year period. These estimates do not allow for laboratory refitting or remodeling costs or for overhead costs above the direct instrument operational costs. Such factors have been excluded because the costs involved are highly contingent on the extent of lab refitting required, the location of the lab, and the wide variations in overhead charges. It should also be noted that the overhead costs will be essentially the same regardless of the lab instrumentation facilities selected. The footnotes to table H-6 provide further qualifying information.

The decision to exclude estimates of the costs of preparing the samples for the analyses has been based on several factors including: 1) lack of information regarding sample types and numbers, 2) the influences of the preparation procedures actually selected on costs, and 3) the fact that the sample prep costs will be essentially the same for each of the analytical techniques considered.

Comparisons of the estimates presented indicate that the plasma emission techniques offer superior economic advantages which are complementary to the sensitivity advantages previously discussed. Although more analyses per day can be carried out with the plasma techniques, the present estimates have been based on the expectation that it will be necessary to analyze each sample via two different sample introduction methods to achieve the required sensitivity. Thus, several elements may be determined by direct nebulization of the aqueous sample solutions. A second set of elements, e.g., $\mathrm{As}, \mathrm{Bi}, \mathrm{Hg}, \mathrm{Sb}, \mathrm{Se}$, and Sn, may have to be determined using the hydride generation method to achieve the required sensitivity (low-threshold effect levels). A similar situation is anticipated for atomic absorption spectrophotometry; direct flame measurements will be adequate for some elements while furnace measurements will be required for others. Analysis in the single-element mode for AAS is clearly more costly. The inclusion of the simultaneous multiele- 
ment AAS estimates is based on present experience at Colorado State University. Conversion of single-element AAS units to the multielement capability can be anticipated in the near future. The long-term cost advantages are obvious.

The restricted capabilities of the electrochemical system tend to remove it from competition except as a supplementary or specialized capability. Primary arguments for the use of NAA or the Xray techniques are based on the ability to directly analyze tissue (solid) samples without dissolution. Unless this capability is important, the cost differentials evident in table H-6 argue against their use.

In view of these data, the use of the ICP-MAES system as the primary instrument appears quite rational, The inclusion of an AAS system and an electrochemical (EC) system as support (e.g., cross-check) techniques should be recommended. The cost estimates given for the AAS and EU units in table H-6 were based on the expectation that each might be the primary lab facility. When their roles are reduced to a secondary (support) level, less sophisticated (high versatility and production) units may be acceptable. The cost of an adequate AAS facility could thus be reduced by $-\$ 8,000$ to $\$ 10,000$ and that for the electrochemical unit by $-\$ 5,000$ to $\$ 10,000$. It should also be noted that these lower cost units could be interfaced to the ICP-MAES computer system preferably using inexpensive (\$300 to $\$ 500)$ microprocessors as buffers.

\section{ESTIMATED ANNUAL SAMPLE THROUGHPUT LOAD}

The above estimates of sample throughput capabilities may be used to approximate annual analytical productivities. These are based on a total of 260 working days per annum minus 60 days for vacations, holidays, downtime, quality assurance time, etc., leaving 200 effective 8-hour days. The projection below is based on the assumption that the primary load will involve the analysis of biological (organic) tissues; such analyses probably represent the most rigorous time/cost requirements,

Using the estimate of 15 to 30 samples per day as the load that can be handled by a sample preparation technician, the annual preparative capability ranges from 3,000 to 6,000 samples. Analysis of these samples for 30 elements (as an upper limit example) using the plasma emission capability involves performing 90,000 to 180,000 analyses. Applying the lowest analytical throughput (1,500 analyses/day; table H-6) suggests that 60 to 120 days would be required for these analyses once the samples were prepared. In essence. this emphasizes a fairly universal observation, i.e., preparation of samples for analysis is often the factor which limits laboratory productivity. Several inferences may be drawn from the above sample preparation and analysis estimates:

1. The analytical capability proposed is sufficient to keep 2 to 4 sample preparation personnel busy if biological tissues comprise the principal workload of the lab.

2 The sample preparation methods used need to be upgraded in terms of throughput per unit time.
3. The analytical facility could be used for additional types of analyses which do not require sophisticated (time consuming) sample preparation operations, e.g., water samples.

Comments on the last two possibilities are appropriate.

Primary factors which affect the sample preparation time/cost requirements include: the elements to be determined and the types of samples. The determination of toxic elements in natural and effluent waters is perhaps the simplest case. The water samples must be filtered and appropriate preservatives added; these operations must be carried out as soon after sample collection as possible and preferably in the field so essentially no lab prep operations are required, Such analyses could occupy the additional time available on the instrumentation. The preparation of animal tissues (particularly liver) is at the other extreme. These contain varying amounts of fatty materials (lipids) which are difficult to decompose by wet oxidation techniques thereby requiring that rigorous conditions be used. Although dry oxidation essentially circumvents this problem, the fact that several elements may be lost by volatilization forces the use of slower oxidative methods, e.g., temperature-programed furnaces, low-temperature ashing units, or sealed (high-pressure) bomb systems. The estimates given above have been predicated on the use of wet oxidation (reflux) techniques in common use; these appear to be less susceptible to problems than the dry techniques. It has also been assumed that complete destruc- 
tion of lipids present is required. An examination of the literature, however, has not produced convincing evidence that this is essential. The point raised is simply: Can the most significant fraction (e.g., >90 percent) of the elements of interest be "extracted" from the tissue matrices via wet digestion without complete destruction of the fat, lipids, or cellulose present? If so, the sample preparation times can be prominently reduced. Finally, it should be noted that the wet ashing of biological materials in microwave ovens shows prom- ise in alleviating the digestion time problem. ${ }^{15}$ Spike-recovery studies carried out when food products were digested via this method are very encouraging. The ability to reduce sample digestion times by a factor of two or more appears to be a reasonable estimate. Factors of this nature must be considered in the final planning stage for the present program.

Adel Abu-Samra. J. S. Morris, and S. R. Koirtvohann. Anal. Chem., 47. 1475 ( 1975). Seealso reports on USPHcontract No. 223-75-2268 and U.S. Patent 4,080,1 68.

\section{ANALYTICAL ACCURACY COMPARISONS}

As emphasized above, the validity (accuracy) of the measurements strongly influences the integrity of any decision based on the results, Any measurement is subject to errors which may be influenced by several factors including the measurement methods used and the analyst responsible. This is the primary reason for stressing the importance of a quality assurance program. The general degree of accuracy that can be achieved with analytical techniques discussed above can be anticipated to be essentially the same for all techniques. This first approximation expectation is based on the following. First, each of the techniques discussed essentially requires the same sample preparation procedures. Since these are likely to prove to be significant (primary?) sources of analytical errors, the errors which may accrue during the preparative steps will be essentially the same for all techniques. Second, the analysts involved can be responsible for the cause or prevention of errors depending on the expertise and caution they exercise. The analyst that tends to use poor technique or judgment when applying analysis method A will, in all probability, do the same for method B; the errors for which he is responsible will be comparable in both cases. Finally, the measurement accuracies of each technique discussed above are generally similar. As a result, there are no clear-cut, easily defensible reasons for suggesting preference for one approach over another on the basis of improved accuracy.

\section{RESEARCH NEEDS ASSOCIATED WITH THE PRESENT PROGRAM}

In the section discussing the criteria involved in the selection of an analytical method, the general importance of being able to identify and measure the chemical forms or oxidation states of several elements was emphasized. This emphasis was based on the fact that all segments of the ecological system are affected to varying extents by the elements (e.g., metals) present. Indeed, the statement that "the life processes of every living cell are conditioned by the types and amounts of metals present" would be accepted by a majority of scientists. Some metals are essential to the health of living systems and, yet, they may also be insidious pollutants because of their generally nonbiodegradable nature. Only a few metals are completely nontoxic at any concentration level; most cause deleterious effects at some exposure level. The ultimate definition of: 1) what constitutes a deleterious effect?; 2) what actually causes it?; and 3) what are the operative threshold-effect concentration levels? cannot be considered trivial problems. It is unfortunate that simply measuring the total amount of a particular element present in an ecological system may, in fact, be only a gross indicator of potential or actual deleterious effects, What is often needed is the identification of the active or functioning forms of the elements in question. Although numerous examples which support this statement can be cited, the fact remains that chemical form is often extremely important.

For this reason, the development and refinement of analytical methods and techniques for the identification of chemical form (chemical speciation), at the trace to ultratrace concentration levels so often of interest, has received considerable attention in the past decade, Although this is 
clearly an important area of research, progress has been slow for several reasons. These include:

1. The analytical techniques most suitable for the determination of chemical form are also generally those which lack sufficient sensitivity for use at the low concentrations characteristic of many elements in biological systems.

2. Biological systems are inherently very complex mixtures of a wide variety of chemical constituents. It is a truism that the complexity of the identification problem is dramatically enhanced by the compositional complexity of the target system.

3. While it can be argued that chemical identification techniques are necessary for the delineation of what chemical forms are biologically or toxicologically important, it appears equally valid to argue that such knowledge on an a priori basis is highly beneficial as a $\mathrm{n}$ aid $\mathrm{i} \mathrm{n}$ focusing the development $\mathrm{t}$ work.

4. The analytical chemical community is often only partially aware of what types of chemical speciation measurements are considered most important by the medical, toxicology, and ecology communities. Similarly, the latter are often only peripherally aware of the most promising emergent analytical technology.

5. The scientific communities involved in this chemical speciation question have often been forced by the pressing circumstances so frequently associated with deleterious effects to expend their efforts on the use of less than satisfactory approaches leaving less time for the required types of development.

Although other contributing factors could be cited, the case in point is simply that the chemical speciation capabilities so badly needed are available in only limited instances. The development of adequate speciation technology should be a central thrust of research efforts involving collaborative efforts between the toxicology, biological effects, and analytical chemical communities. Governmental and private funding agencies should strongly encourage these efforts, Because propogation of chemical speciation developments will ultimately be essential to the success of the overall monitoring program, the laboratory facilities available at the outset should take advantage of present capabilities. Moreover, the prime stance of the program should be that of providing feedback to the relevant scientific communities as one means of focusing speciation research efforts on those elements which may appear to be more important from the effects standpoint.

Technology presently exists for the differentiation between the organic (alkylated) and inorganic forms of the toxic elements: arsenic, lead, mercury, and selenium. These rely on the fact that the inorganic forms can be easily converted to gaseous forms (mercury vapor and the hydrides) by reaction with borohydride while the organic forms must first be photodecomposed by exposure to ultraviolet (UV) radiation, Thus, analysis of the gaseous reaction products before and after UV irradiation provides measurements of the relevant organic and inorganic concentrations. This chemical differentiation approach has been interfaced with atomic absorption and emission spectrometry as the measurement tools; inclusion of this capability in the laboratory is recommended.

Similarly, the ability to differentiate between the possible oxidation states for some metals is important, e.g., As(III) versus $\mathrm{AS}(\mathrm{V})$ or $\mathrm{Cr}(\mathrm{III})$ versus $\mathrm{Cr}(\mathrm{VI})$, Such differentiation for $\mathrm{As}, \mathrm{Cr}$, and some other metals can be accomplished by use of selective oxidation-reduction (redox) reactions. The redox reactions may be controlled by proper selection of chemical reagents or by judicious use of electrochemical (polarographic or voltammetric) principles. Electrochemical measurements can also be used to differentiate between electroactive and nonelectroactive (chemically bound) forms of some elements and between "labile" and "nonlabile" bound forms of some elements. In this context, labile and nonlabile refer to the thermodynamic and/or kinetic stabilities of the metalligand systems in question. Although this type of differentiation may be only semiquantitative or semiempirical in nature, such measurements have been shown in some instances to be pertinent in experimentally defining bioavailability, transfer mechanisms, transfer rates. etc. It is certain that research must still be done to further delineate the diagnostic potentials of such types of differential measurements, Present knowledge seems sufficient, however, to support the inclusion of polarographic and voltammetric instrumentation in the laboratory facilities for "lability" and/or oxidation state measurements.

A limited number of other possibilities exist for chemical speciation measurements. These are largely very specialized in terms of applicability to certain types of samples and will not be discussed herein. It is certain that the need for chemical speciation exists and that its importance will become even more apparent as more definitive 
monitoring programs evolve. It is also certain that no single analytical technique for speciation or any other purpose will be a universal panacea. The ultimate solutions to the majority of specia- tion problems will involve the application of some combination of chemical and physical principles, The research programs most cognizant of this are most likely to be successful.

\section{GLOSSARY OF ABBREVIATIONS}

AAS-atomic absorption spectrophotometry

AES-atomic emission spectrometry

ASV_anodic stripping voltammetry

cc-cubic centimeters

cm--square centimeters

$\mathrm{DCP}$-direct current plasma

DPP_differential pulse polarography

FC-Faradaic or analytical current

$\mathrm{Ge}(\mathrm{Li})$ - designates a germanium crystal which has lithium drifted into it to give it uniquely defined properties

gin-gram

ICP-inductively coupled (radiofrequency) plasma

MAES-multielement atomic emission spectrometry

Mev--million electron volts

ml-milliliter

NAA-neutron activation analysis
$\mathrm{Ng}$ - nanograms - one billionth of a gram

PES-plasma emission spectrometry

PIXE-proton induced X-ray emission (spectrometry)

$\mathrm{ppb}$ - parts per billion ( $1 \times 10^{-9} \mathrm{gms} / \mathrm{gm}$ on a weight basis or $1 \times 10-\mathrm{j} \mu \mathrm{g} / \mathrm{ml}$ on a weight per unit volume basis)

$\mathrm{ppm}$ - parts per million $\left(1 \times 10^{-6 ;} \mathrm{gm} / \mathrm{gm}\right.$ or $1 \mu \mathrm{g} / \mathrm{ml}$ as for $\mathrm{ppb})$

$\mathrm{Si}(\mathrm{Li})--\mathrm{a}$ silicon crystal which has lithium drifted into it to provide unique physical properties

SMS—solids mass spectrometry

$\mu \mathrm{g}$ - one millionth of a gram

$\mu \mathrm{l}$-one millionth of a liter

$\mu \mathrm{m}-\mathrm{one}$ millionth of a meter

XES-X-ray emission spectrometry

$\mathrm{XRF}$-X-ray fluorescence (emission spectrometry) 


\section{Analysis of Foods for Radioactivity*}

by Naomi H. Harley

\section{INTRODUCTION}

The analysis of foods for radioactivity should not be considered as a primary defense against human intake. The first indication should always come from information on releases or from measurements of radioactivity in the airborne or waterborne releases. Once the existence of contamination has been established then the foods can be analyzed to evaluate potential hazard to man.

In contrast to most other pollutants the effects of radiation are considered to have a linear response regardless of the level, thus, there is no threshold and no absolutely safe limit. Instead it is necessary to set some lower level below which the radioactivity in foods is no longer of interest as compared with other sources of radiation or other hazards of life. The analytical significance of this is that the lower limits of detection for radioactive substances have been brought down to very low levels and the simple yes or no testing for acceptability that satisfies regulations for many other pollutants in foods cannot be used.

The radionuclides of interest in the case of contaminating events are almost all present now in foods in measurable quantities. Short-lived nuclides are the exception and the transuranic elements are only present at levels that require con- siderable effort in analysis. Since most of the radionuclides are already present, measurements made for background information should produce a numerical answer, not merely an indication that the amount is less than some pre-set value. The accumulation of background data provides a valuable baseline for evaluating excursions following a contamination event, The natural activity data are equally valuable since the amount of information on food concentrations is presently insufficient for valid comparisons with manmade radioactivity.

This report will describe the requirements and considerations for establishing a system to produce acceptably accurate measurements of radionuclides in foodstuffs. The basic concepts will be described, but to maintain the necessary brevity, the detailed procedures that might be used will be given only by reference.

During the preparation of this report, FDA has proposed certain recommendations for State and local agencies on Accidental Radioactive Contamination of Human Food and Animal Feeds. This material appeared in the Federal Register for December 15 , 1978, page 58790, and is interesting background material for this topic.

\section{CLASSIFICATION OF RADIONUCLIDES}

The most useful classifications of a radionuclide are those based on the characteristics of the radiation emitted and by the identity of the chemical element. The former is both a guide to the nature of the hazard involved and to the measurement required. The chemical species (e.g., element, oxidation state) regulates the metabolic pathways in the biosphere as well as the nature of any chemical separations required in the measurement procedure.

* Exrerplfromano I A WorkingPatperentitled "Arlili siscot Fourts for Radioactivits." A completecopy of the paper canbe?

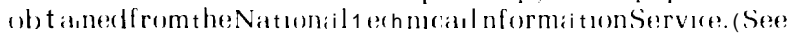
ispp.].)
The emitted radiations are generally grouped as alpha $(\alpha)$, beta $(\beta)$, and gamma $(\gamma)$. Alpha radiation is characteristic of the natural and artificial radionuclides of high atomic weight and consists of energetic particles with very low penetrating power. Its hazard is significant only within the body, where alpha-emitting nuclides can irradiate specific sensitive tissues. Beta radiation appears in both heavy and light natural and manmade radionuclides, and consists of electrons possessing kinetic energy and having modest penetrating power. Gamma radiation is pure electromagnetic radiation and is extremely penetrating. Thus, it can be a hazard externally as well as when it is 
present in the body. For the present purpose, we are only concerned that the penetrating nature of gamma radiation allows its direct measurement in foodstuffs, while alpha and beta emitters generally must be separated from the bulk constituents of the sample before measurement is possible.

There are other processes in radioactive disintegration that produce emissions. Alpha emission is usually accompanied by low-energy gamma rays that may be used for measurement. X-rays can be produced by electron capture and some gamma emitters decay by internal conversion, a process where a fraction of the gamma rays are converted to monoenergetic electrons. These processes do not really modify our measurement concepts but the decay modes of the significant radionuclides must be known for their accurate measurement.

The physical half-life of the radionuclide tends to control its persistence in the environment. For example iodine-1 31, with a half-life of about 8 days, is important for only a few weeks, while cesium-137, with a half-life of about 30 years, may be a problem for centuries.

A third classification that has some value is the source that produced the radionuclides. Knowledge of the source of radioactive contamination gives a good indication of the nuclides that are to be expected in the sample. This is of considerable assistance in planning the analysis since requesting a complete analysis for all radionuclides or even for all types of radioactivity in a single sample would lead to a lengthy and expensive opera- tion. Many radionuclides are not potent health hazards, particularly those that are not metabolized by the body. The general groups of nuclides to be expected include the natural activities, specifically radioactive potassium and members of the uranium and thorium series, and the artificial fission products, transuranic elements, and other activation products that result from nuclear weapon explosions and nuclear reactor operations.

Fission products are a very complex mixture at the time of formation but the short-lived radionuclides die out rapidly and the mixture becomes simpler within a few days, The transuranics (plutonium, americium, etc., formed by activation of the basic fissionable material) are of some interest because of their high toxicity when incorporated into the body but present evidence indicates that their uptake through the gut is relatively small and that dietary intake is not a significant problem. This should be true particularly if the relative hazard of other radioactive contaminants probably present in the same sample is taken into account, The other activation products are frequently elements that make up steel or other metal containers or structural elements. Radioactive manganese, chromium, cobalt, zinc, and iron are particularly common and result from interactions of the materials with neutrons released in the nuclear reaction. It is worth pointing out that contamination of foodstuffs with single nuclides is extremely unlikely, and that more than one member of any group will probably be present in any sample.

\section{DISTRIBUTION OF RADIONUCLIDES}

The source of the radionuclides involved will generally control their distribution in the environment and their consequent transfer to the food chain. The sources considered here will include natural radioactivity, releases from operation of nuclear reactors and processing plants, and fallout from nuclear weapons tests.

Natural activity may be of concern when it is enhanced by man's intervention, say by mining to bring material to the surface and processing the ore to yield either products or wastes that may concentrate the radionuclides. Good examples are radium in uranium tailings, in phosphate rock waste, or in slags from phosphorus production, Radium may enter the food chain by dissolving in ground water and transferring through plant roots.
Nuclear reactors in normal operation release chiefly the radioactive noble gases that are not of interest in considering foods. Reactors do contain large inventories of fission products, transuranics, and other activation products, however, and accidental releases can contaminate vegetation by deposition or through the water pathway. Gaseous releases would most likely involve the volatile elements such as iodine and tritium or those with volatile precursors, such as strontium-90 and cesium-137, Aqueous releases would follow failure of the onsite ion exchange cleanup system and any water-soluble elements could be involved.

Processing plants could also have either gaseous or aqueous releases, but only fuel reprocessing is likely to be a significant contributor. In 
this case, the fission products are aged before processing and iodine and the gaseous precursor radionuclides are not released. Tritium and carbon-14 are the major airborne products, while the waterborne radionuclides are the same as for reactors.

Atmospheric nuclear weapons tests distribute their fission products, transuranics, and other activation products globally, with local deposition being more or less, depending on the size of the weapon and the conditions of firing (high altitude, surface, underground).

In summary, the deposition of airborne material on vegetation or on soil is the route by which foodstuffs become contaminated and the subsequent behavior of the radionuclide is controlled by its chemical nature, including solubility and plant or animal metabolism.

\section{CONTAMINATION OF FOODSTUFFS}

Contamination of foods can occur either through atmospheric deposition or by transfer with water. In the first case it is possible for the radioactive material to be in the form of insoluble particulates rather than in a more available form where it will follow the chemistry of the elements involved. A knowledge of the pathway is not absolutely necessary but it does assist in deciding on the proper preliminary treatment of the sample of evaluating exposure. For example, surface contamination may have a different significance than the same material present in a plant through root uptake.

Since pathway information is not always available $\mathrm{i} t$ is generally considered proper to measure radioactivity in samples that have been prepared as if for eating, so as to approximate the true expected intake. This will usually result in stripping off or washing off of a considerable fraction of surface contamination. Cooking is generally not part of the preparation, as the mode of cooking and the use o f j u ices, cooking water, and the like cannot be predicted,

Milk is often recommended as an indicator food for studying radioactive contamination. It has many advantages:

1. It is available locally at most desired sampling locations.
2. It is marketed rapidly, so that short-lived radionuclides. such as iodine-131, can be evaluated.

3. It is a major diet component in the United States, both directly and as an ingredient of prepared foods.

4. There is a lot of background information available on previous contaminating events.

It is worth noting that some of these "advantages' are the factors that contribute $t$ o the role of milk as a source of human exposure.

Milk is a poor indicator of many contaminants. The natural activities, the transuranics. and the activation products have relatively low concentrations in milk. The first two are low because of poor biological transfer and the last because their pathways are almost entirely through the aquatic or marine food chain. Thus, the best approach is to know what is in the environment through other monitoring systems, and to design the food analysis program to fit the circumstances.

Other monitoring data are also necessary in fixing the geographical extent of a contaminating event. As a general rule, nuclear tests are global in radionuclide distribution, with enhanced levels near the test site. Releases from other nuclear operations tend to be more local in their effects and the food-monitoring plan can be modified to suit.

\section{SAMPLING}

The mechanics of obtaining representative food samples will not be considered here since the procedures are common to all types of food analysis. There are certain points that must be considered however. The first is whether the measurements are being made to determine human intake or the source of the contamination. In the latter case the early approach used by $\mathrm{FDA}(1)$ for radioactivity is approp $\mathrm{r}$ i a te. There, each sample was identified as to its place of origin. For evaluating intake $\mathrm{i} t$ is possible to collect total diet samples for a particular population group and to measure the radioactivity in this composite diet. This approach has been described by $\operatorname{FDA}(2)$ and by $\operatorname{EPA}(3)$ and is normally applied in institutions where mass feeding is carried out. A more elaborate procedure is to simulate a total diet by measuring a number of component food classes selected on the basis of 
statistical information regarding consumption. This approach was originally used by the Atomic Energy Commission(4) and was applied originally to three major cities. This approach does allow identification of specific food types that are contaminated but requires much greater effort and cost in analysis.

The use of indicator foods as an intermediate type of monitoring is widespread. As mentioned previously, most of the systems depend on the sampling of milk which is available in most parts of the country either with specific information as to place of origin or the general area of the milkshed. Part of the reason for using milk is the ease in sampling but it also has significance as a primary food for the youngest and most susceptible population group and it also does tend to pick up several of the fission product nuclides of dietary significance such as radiostrontium, radiocesium, and radioiodine. Most other foods give limited geographical or seasonal coverage and are less satisfactory.

The preservation of samples in the field during transport and in the laboratory awaiting analysis is only an esthetic matter in the case of radionuelides. Decomposition processes do not change the radioactivity, and sample contamination by radioactivity is unlikely. Thus, freezing, formaldehyde addition, or any other method that will maintain the sample is adequate. Any additive should be checked to assure it does not contain significant amounts of the radionuclide sought.

For the purpose of this report we will assume that an adequate quantity of a representative sample is available for analysis and that another portion is available for storage, either permanently or until the analytical results are accepted as satisfactory.

\section{SAMPLE PREPARATION}

The measurement of radioactivity is a physical process and it is most efficient when the radioactivity from a relatively large sample can be placed close to the detector. This means that direct measurements of bulk samples are only useful at relatively high levels of contamination and that most measurements are preceded by preparation and possibly chemical separation to reduce the bulk of the material and to improve the efficiency of the measurement.

As mentioned previously, sample preparation may include removal of inedible portions of the foods or those portions not generally eaten. For example, citrus rinds, apple cores, outer leaves of leafy vegetables, and aboveground portions of root vegetables would normally be discarded. The general goal is to prepare the foods as if for cooking or consumption.

Foods generally have a high water content and a primary method of bulk reduction is drying at room temperature, at elevated temperatures, or freeze drying, Most of the radionuclides of interest are not volatile under these conditions and losses must be considered only for elements such as tritium and iodine. The dried material can be reduced further by ashing at elevated temperatures, by cold ashing with activated oxygen, or by wet ashing with oxidizing acids. The sample dryashing process is most likely to lead to loss of volatile elements but with care even cesium, polonium, and lead can be retained, The other proc- esses should not lead to losses of elements of interest with the exception of iodine and tritium, mentioned above, and of carbon.

Another approach that is essentially one of reducing bulk is to extract either the original sample or the dried or ashed material with acids or other solvents and thus remove the desired elements from the bulk of the sample. This requires considerable testing beforehand to be certain that the process operates in the desired manner.

All of these procedures reduce the bulk of the sample and in the case of extraction may also separate the desired constituents from some of the remaining inert material. They do not, however, separate the radionuclides of interest completely from the other radionuclides present in the sample. Such separations will be covered in the next section, but they may not be necessary if the measurement technique can provide both qualitative and quantitative information. In most cases this limits the possibilities to gamma spectrometry on the prepared sample.

If the samples are going to be subjected to chemical analysis, the sample preparation must include the dissolution of the dried or ashed material. This has already been done, of course, in preparing the wet ashed or extracted solutions. The radionuclides of interest in ashed foods should be soluble in strong acids if ashing temperatures have not been excessive, and this treatment is usually accepted. If there is concern that 
insoluble particulate may be present, it is necessary to use more drastic methods such as fusion to bring the sample into solution. This should not be necessary, however, if human hazard is the problem under consideration.

At the time of preparation, if not before, the basis of measurement must be established. Depending on the use of the data, wet weight, dry weight, ash weight, volume, or even numerical count (e. g., eggs) has to be determined. Frequently, this may have to be done in the field, but fortunately, relatively crude measures are adequate,
Since unforeseen questions often arise, it is recommended that as many of these quantities be measured as is possible.

The requirements for measurement of radionuclides in foods are such that sample preparation is best handled by the group responsible for the rest of the analysis. The chief difference from other types of analytical work is the initial sample. The usual range is from 1 to $20 \mathrm{~kg}$, and the reduction of this amount of material is a specialized problem.

\section{RADIOCHEMICAL SEPARATIONS}

Radiochemical separations are required to isolate the desired radionuclide both from the remaining bulk constituents and from other radionuclides which would interfere in the measurement. In addition, it is necessary to convert the final product to a form suitable for presentation to the counter. This may involve elect redeposition, precipitation, or other processes. There are a number of manuals giving the details of specific radiochemical procedures and these details will not be repeated here. There are a few generalities however that may be of interest.

The actual mass of radionuclide that is measured is almost always vanishingly small. This means that many of the normal chemical reactions used in analytical chemistry will not take place; for instance precipitates will not form. For this reason it is common to add a few milligrams of carrier material which is preferably the inert form of the same element. Where this does not exist it is frequently possible to use similar elements as carrier, such as the substitution of barium for radium. The inert form then follows normal chemistry, carrying the radionuclide with it. It is also worth noting that even when the separation technique does not depend on the mass of element present, a carrier may still be useful in preventing unwanted coprecipitation or absorption on glassware.

Because of the high degree of purification required in radiochemistry, it has been customary to make a number of repeated separations either identical or different to insure purity. To carry these out in a reasonable time it is better to lose a small amount of the nuclide sought while removing a large fraction of the undesired material rather than retaining all of the nuclide and much of the undesired material. Fortunately, it is possi- ble to measure these losses in analysis and to make a correction at the end. One approach is to measure the carrier at the end of the analysis either by gravimetric or instrumental analysis. If there was none of the carrier substance present in the initial sample, the fraction recovered will be equal to the fraction of desired radionuclide recovered. Where carriers are not available or where they are present in variable amounts in the original sample it is frequently possible to use radioactive tracers, that is radioactive isotopes of the same element or a similar element. The fraction of the amount added that is left at the end of the analysis can be used to determine the recovery of the radionuclide sought.

In the chemical separations there is considerable use of classical analytical chemistry based on precipitation and in most cases it will at least appear as $a$ final collection step to put the desired radionuclide in a condition suitable for counting. In addition the general techniques of ion exchange, liquid extraction, distillation, and electrolysis are used.

Since many of the separations in radiochemical analysis are carried out in small volumes the centrifuge is widely applied, It has become common practice to redissolve and reprecipitate rather than to wash precipitates carefully. This may be repeated several times very rapidly and will generally give good decontamination (separation from other radionuclides). As noted above, where the element sought and the contaminant have similar properties it is preferable to use two different precipitations, a precipitation followed by an extraction, or any two widely different steps to achieve good decontamination.

One process that is frequently of value is called scavenging. This term is usually applied to a pre- 
cipitation carried out to remove contaminating radionuclides after the bulk matrix of the sample has been removed. The process involves the addition of a group carrier, precipitation, and discarding of the precipitate. A typical example would be an addition of iron carrier followed by hydroxide precipitation to remove rare earths and other heavy metals in a determination of an alkali or alkaline earth. Frequently the scavenging procedure is repeated to improve decontamination, but it is only rarely that the scavenge precipitate is redissolved and reprecipitated to recover any of the desired constituent that may have been absorbed.

After suitable radiochemical separations have been made, it is necessary to collect and mount the sample for counting. This is most often done by precipitation or by elect redeposition. In the section on measurement, the requirements for sample preparation are discussed: for example, the sample area and mass should be reproducible and the sample should be mounted on a sample holder identical with that used for the counter standard. Since it is frequently necessary to determine the weight of precipitate for recovery determination, this factor must also be considered.

The selection of a mounting technique is usually a compromise between convenience and the counting requirements. Besides consideration of the type and energy of emission, practical matters such as the counter size and sample mounts available must be weighed,

In certain cases, the total amount of sample available may be limited and analysis for several radionuclides may be required. Procedures should be on hand for the sequential analysis of single samples, even though separate samples are used for routine work.

\section{MEASUREMENT}

The method of measurement to be selected depends on the type of radiation, the form of the sample, and to some extent on the amount of radioactivity. It is necessary that the complete analytical procedure be designed so that the sample is brought to a suitable form for the equipment and conditions that exist.

It is possible to measure the total gamma, total beta, or even the total alpha activity on a sample of food, Unfortunately, such data are valueless in estimating human exposure. The accuracy of the determination is very poor, natural potassium usually interferes, and the chemical and radiation characteristics needed to evaluate possible hazard are not known. It is possible, however, to set a particular total activity level as a screening level for a specific food. If the measured value is below the screening level, no analyses for individual radionuclides are performed. In such a case, the measurements should be considered as internal data only and the numerical results should not be published. Any report should merely list the samples as having activities below the stated screening level.

Qualitative identification of radionuclides on original samples of foods is limited to gamma emitters at relatively high levels. The sensitivity can be increased if some bulk reduction, as described under "Sample Preparation," is carried out. The identification of alpha and beta emitters depends on radiochemical separation for element identification and the measurement of energy or half-life on the separated material for radionuclide identification. The latter step may be omitted if other considerations limit the possibility to a single radionuclide.

The equipment available for quantitative measurement of radioactivity is sufficiently sensitive for all foreseeable cases. Instruments for detection of the three major emissions are described here, and their applications are shown in table 20 (chapter VIII) in terms of the detection limits for various radionuclides.

It is worth pointing out that a number of the instruments described are not commercially available. They have been in the past, but the low demand has removed them from the market, The necessary electronic components are available but the mechanical assemblies for the detectors are not, and the larger laboratories tend to build their own systems.

Alpha Emitters. The measurement of alpha activity is best carried out on a very thin sample to avoid self-absorption of the alpha particles. This is even more true for spectrometry since degradation of the original alpha energy will give a spectrum with poor resolution. The measurement of the total alpha activity can be carried out either in thin-window counters or by scintillation counting with zinc sulfide phosphor. Both techniques have high efficiency but the scintillation method can give a considerably lower background with a consequently lower limit of detection. Unfortunately, neither system is readily available as a 
complete unit and must be assembled from commercially available components. A somewhat less sensitive technique is to use the liquid scintillation spectrometer described in the next section.

Alpha spectroscopy also has two possibilities, the Frisch grid ionization chamber or the silicon diode solid-state detector. The Frisch grid unit can handle large area samples but is slightly poorer in resolving closely separated energies. On the other hand, the silicon diodes available are all quite small and can only count samples of about 1cm diameter with high efficiency. This is not a serious limitation, since the Frisch grid systems are not presently available in this country. This means that the form of the sample presented to the counter is limited. Most metals are electrodeposited onto small smooth discs of stainless steel. nickel, or platinum, with evaporation of pure solulions being used in some cases.

A specialized technique is available for measuring radium-226. by means of its gaseous daughter product radon-222. If the sample can be put into solution and stored for about 3 weeks the radon-222 will be close to equilibrium (equal activity) with the radium-226. The gaseous radon can then be transferred to a scintillation counter or an ionization chamber for measurement of its alpha activity. This is rather specialized, and the equipment is not listed in this report since radium-226 can be determined by other procedures. For example, separation and counting as the sulfate or chromate using barium as carrier is quite adequate.

Beta Emitters. Beta counting is a little more flexible in the mass of material that can be present at the time of counting. This is true for higher energy beta emitters but carbon-14 and tritium present a problem. Since each individual beta emitter gives off particles with a range of energies from zero to a characteristic maximum, beta spectrometry is not possible for food samples. Some qualitative information may be obtained from absorption measurements, which allow an estimate of the characteristic maximum energy of single emitters or simple mixtures (5).

The available counting equipment for quantitative measurement includes geiger counters, proportional counters, and scintillation counters. The geiger counter is relatively inexpensive and requires only simple electronics but is not popular and is generally not available as a counting system. The thin-window proportional counter is used widely and has both reasonably high efficiency and low background. For low-level samples the background can be further reduced by antico- incidence techniques. These add to the complexity and cost of the system but are sometimes necessary.

Scintillation counting can be performed in two ways. Solid scintillators can be used for counting chemical precipilates collected on filter papers and liquid scintillators can be used whenever the sample can be made miscible with the scintillating solution itself. This can even be done with solids by suspending them in a scintillating gel. The advantage of liquid scintillators is their high efficiency, even for the low-energy emitters carbon-14 and tritium. Scintillation systems for counting precipitates are not commercially available at present. There are, however, many liquid scintillation systems on the market. most of them with automatic sample changers. They enjoy a good demand in medical and biological studies with tritium and radiocarbon. These use high levels of activity and short counting time. The better systems also have a provision for rather crude spectrometry. They can distinguish qualitatively and quantitatively among carbon-14. tritium, alpha emitters, and higher energy beta emitters.

Gamma Emitters. Gamma rays are so penetrating that the detector must have a considerable mass to absorb enough to produce a response. For spectrometry, complete energy absorption is required, so solid detectors are most useful. Sodium iodide is a popular detector, since crystals can be fabricated in large sizes and the crystal is transparent to the scintillations produced by radiation. Sodium iodide is used for most of the gamma counting today, and is still useful in spectrometry. It has high efficiency but poor energy resolution and is now applied to samples where some separation has taken place. Milk is a good natural example, as the metabolism of the cow removes most gamma emitters other than isotopes of cesium and iodine.

More complex spectra can be resolved with the solid-state germanium diode detector. A comparison of a sodium iodide with a germanium diode detector spectrum is shown in figure I-1. It is possible to resolve the sodium iodide spectrum of a sample containing several radionuclides but this requires a sizable computer and very careful work to allow for drift in instrument characteristics and for the possibility of other radionuclides than those sought being present. The diode detector gives much better resolution and present spectrometer systems frequently include sufficient computer capacity to resolve the resulting spectra and produce quantitative data. At the same time, interferences from radionuclide im- 
Figure I-1 . Comparison of Gamma Spectra Taken With a Sodium lodide Detector (Upper Curve) and a Germanium Diode Detector (Lower Curve). Note that the main single peak in the sodium iodide spectrum is resolved into 9 peaks for 6 radionuclides with the germanium diode spectrum "

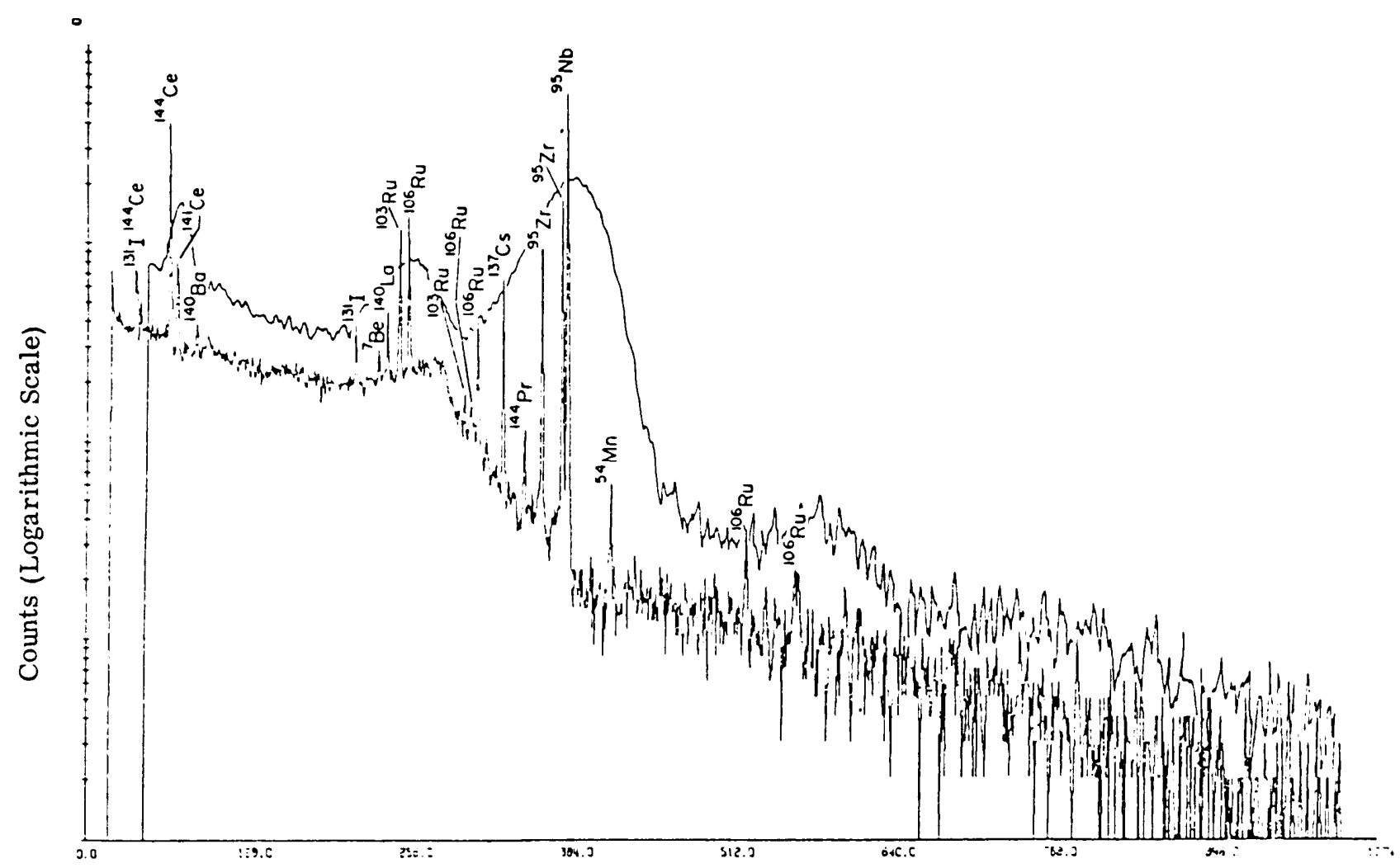

purities are greatly reduced compared to spectra from sodium iodide detectors. The efficiency of the diode is low and, for many analyses, a spectrometer can only be used for one measurement a day. Another disadvantage is that the detector must be kept at liquid nitrogen temperature to maintain its detection capability. Newer diodes have been developed that do not require storage at liquid nitrogen temperatures; however, they must be cooled during measurement. Since the system is in use most of the time, this may not be a significant advantage.

Diode spectrometers may also be used to measure the low-energy gamma-rays that accompany alpha emission. This allows direct measurement in some environmental samples, but the levels in foods have not been high enough for this technique.

General Requirements. The choice of a counting procedure depends on the precision required. In turn, the relative precision of a quantitative counting measurement is inversely proportional to the square root of the number of counts obtained. Thus, any improvement in precision must be obtained by increasing the number of counts. This can be done by using larger samples, by counting for longer times, or by using counters with higher efficiency. A secondary improvement is possible for low-activity samples by decreasing the background. Each of these improvements has some drawback, and selection of the optimum balance requires a degree of experience to weigh 
cost, manpower, and quality. For example, handling larger samples increases the effort in sample preparation and radiochemistry while longer counting times require more counters, and increased counter efficiency or lower background is expensive.

Counting-room operation requires the maintenance of detailed records on standardization, background, and sample measurement. This information can frequently allow the recovery of bad data or the correction of calculational errors.
In addition, maintenance of control charts(5) will signal when instrument problems arise.

Experience with modern nuclear instrumentation has been good, and downtime of the order of 5 percent is common. This requires that service be available immediately or the next day following a breakdown. The increasing complexity of measuring systems tends to preclude in-house servicing in most cases, but some diagnostic capability and competence in minor repairs is very valuable.

\section{CONTROLS}

An analytical laboratory carrying out measurements of radioactivity in foodstuffs will require a program to document the validity of the measurements, This is necessary in legal cases and is also highly desirable when data are being presented for use in decisionmaking. A suitable program of quality control should be carried on in addition to the necessary calibrations and standardizations.

Calibration is the determination of the relationship between a desired quantity and the response of a particular instrument. In the case of radioactive materials this may mean that the instrument must be calibrated with each radionuclide to be measured unless there is evidence that the instrument response is independent of the energy or other characteristics of the radiation. Alternatively a complete response v. energy calibration may be substituted. These calibrations, to have a legal standing, should probably be traceable to the U.S. National Bureau of Standards or comparable authority. This turns out to be a requirement that is far from trivial in the effort required.

Fortunately, a complete calibration is not required at frequent intervals and, depending on experience, may not be needed oftener than every few months. In the meantime of course it is necessary to have assurance of the proper operation of measuring equipment but this can be done with simple standards or even with samples that are reproducible over a period of time. For example, a simple counter standard may be run every morning before starting operations just to be sure that the counter is working properly. Similar standards should also be available for checking spectrometer energy response.

An ideal quality control program should include the checking of the complete procedure from sample preparation through measurement.
This requires that standard samples be available for testing the full procedure. Additional controls would include running of blind duplicate samples to test reproducibility of analyses, and blank samples to check on the possibility of laboratory or reagent contamination. These three types of samples should make up at least 10 percent of the laboratory output if the quality of measurement is to be followed closely. It is also necessary that the data be published with the ordinary laboratory results and that any deviations from the expected results should be used to make suitable corrections in laboratory operations.

There is always some danger that a laboratory may drift out of control in spite of an adequate internal quality control program, This is possible since an internal program may emphasize consistency while the absolute values drift. Therefore, some part of the quality control effort must be devoted to intercomparisons with established groups, such as IAEA or EPA. This should be done on an annual basis, at least.

It must be noted that standard samples are frequently not available, and recourse must be had to "spiking," which is the addition of a known amount of a radionuclide to a sample that is relatively free of that nuclide. Recovery of the spike is not necessarily a good test of a chemical procedure, since a natural sample may be more difficult to analyze.

A final component of a good quality control system is a careful, responsible review of all the data produced. This means checking the arithmetic, knowing the characteristics of the equipment and, hopefully, having a sixth sense that recognizes that certain results do not look right. This is especially true when relying on automatic counters or on computer processing of the data. 


\section{STAFF AND FACILITIES}

A minimum facility could be designed around a staff of six, including a B.S. or M.S. senior chemist with experience, a B.S. junior chemist and three technicians for chemistry, sample preparation, and counting-maintenance, A- secretary-administrative assistant could handle reports, local purchasing, and similar duties. With this small staff, considerable versatility and flexibility would be required.

The measurement of radioactivity in foods requires fairly extensive facilities to handle the varied analyses that might be called for, In addition to a modest office space, separate rooms would be necessary for sample preparation, wet chemistry, measuring equipment, and for maintenance support and storage. The first two laboratory rooms would require hoods, chemical benches, and laboratory safety devices such as showers and eye fountains. Since the samples to be measured are normal foods there are no special requirements for radiation safety, If the samples were radioactive enough to be a personnel hazard in the laboratory they certainly would not require this type of precise measurement.

If the laboratory is to be in continuous operation it is most likely that the principal chemical and measurement systems will have to be available at least in duplicate. This and similar considerations lead to the conclusion that a certain minimum size and sample throughput are not necessary if the radioactivity laboratory is part of a larger operation which can furnish support. One continuing problem is the need for electronic maintenance of radiation measuring equipment. This is rather specialized and must be considered either when staffing the laboratory or in locating it where such services are readily available.

It is not always possible to purchase the ideal counting equipment for a particular purpose. The total demand for many systems is small and commercial instrument makers are not interested. Large laboratories can make some of their own equipment but this is not possible for the group described here. The construction of alpha and beta radiation detectors requires services of a first-class machinist plus sufficient electronic know-how to transfer the detector signal to the available commercial equipment. Where such ca- pability already exists in the overall organization, it may be fruitful to copy advanced noncommercial instruments, but staffing specifically for this function is probably not economical. Thus, most small laboratories must operate with the lessthan-optimum commercial equipment,

If significant uses are to be made of gamma spectrometry there will be a need for at least modest computing facilities. These can be part of the purchased spectrometer system but this is an expensive method if suitable computer time is available otherwise. The former approach is used here, and it might be noted that the spectrometer computer has capacity for other work.

Additional space is required not only for stocking necessary reagents and materials but also for storage of incoming samples and for storage of residual material from samples that have been run. As a general rule it is desirable to take a larger sample than would be required and to set aside a portion of this for possible contingencies or if the data are later brought into question.

The output of a group this size should run between 1,000 and 4,000 samples ${ }^{1}$ a year, depending on the difficulty and the activity level, One limiting factor is that, at background levels, each counter can only turn out one or two samples per day. Following a contaminating event, it should be possible to process smaller samples and to count for shorter times, both of which would allow higher output. It might be possible to add 3 more laboratory staff plus 50 percent more space and equipment dollars and essentially double the output. Further increases would probabl run into other bottlenecks due to the need for added support.

Table I-1 details the costs for space modification and furnishings $(\$ 140,000)$, for equipment $(\$ 200,000)$, and for supplies $(\$ 20,000)$. These costs are based on a building in place, lights and partitions in place, and utility stubs in each room, It is also assumed that building services are provided.

IA sample is defined as a completegamma spectral analysis or a single radionuclideanalysis that requires preparation plus radiochemist rv. 
Table I-1. - Costs for Space, Furnishings, Equipment, and Supplies

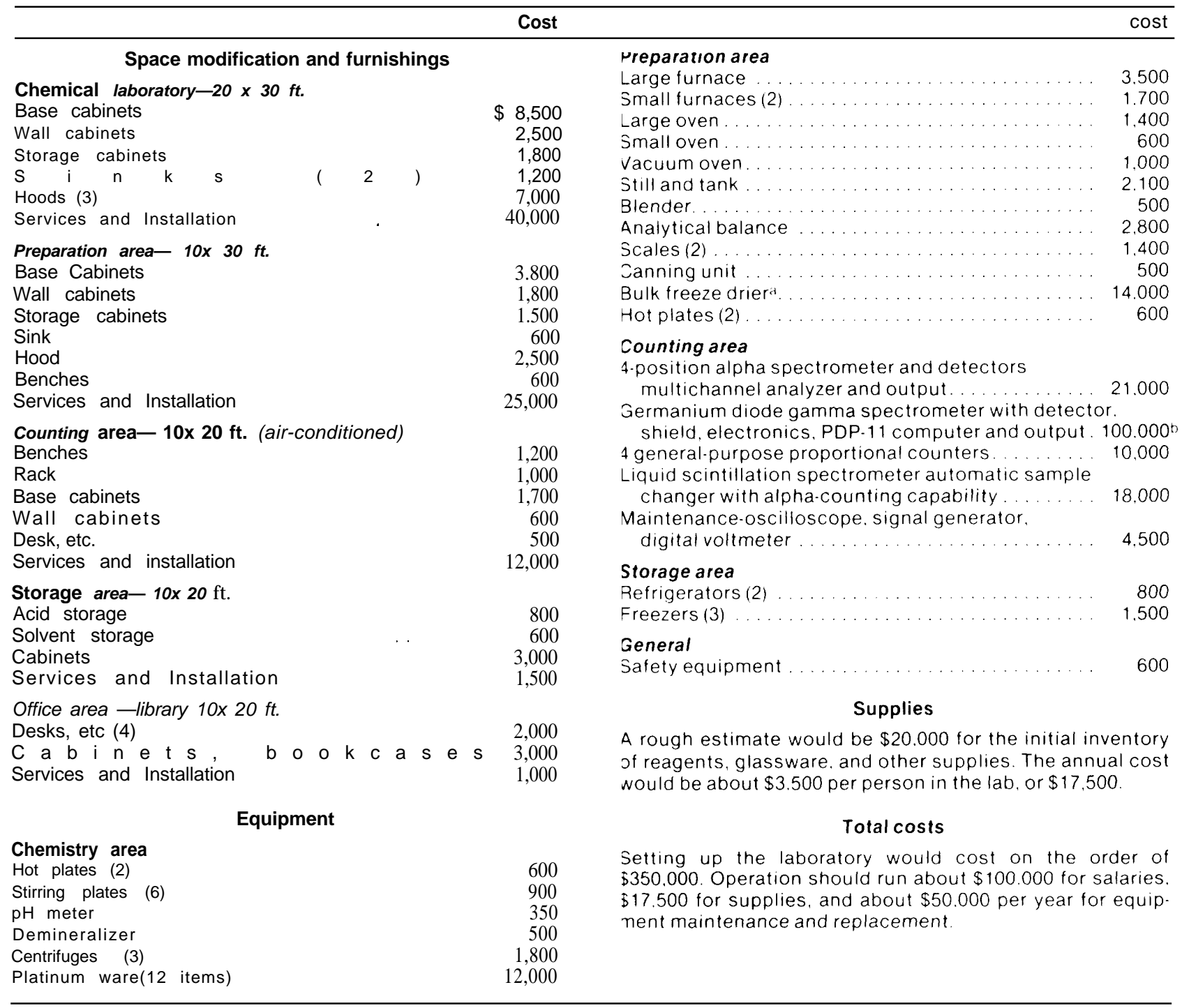

doesirable but not absolutely essentral

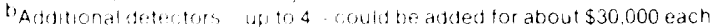

\section{APPENDIX I REFERENCES}

1. E. P. Laug et al. "radioactivity in Foods," Journal AOAC, 44, 52.3-30 ( 1961), also "Strontium-90 Analyses of Human and Animal Foods, Radiological HealthDota 1, 36-40 (May 1960).

2. Teen-Ager Diet Survey, Food and Drug Administration, Rordiological HealthData 4, 18-22 ( 1963)

3. R. E. Simpson.E. J. Barat ta, and C.F.Jelinek. "Radionuclides in Foods: Monitoring Program. "Rodicution
Dataand Reports 15, 647-56 ( 1974). ('This is a later report that summa rizes much of the EPA program. )

4. J. H. Harley and Joseph Rivera, Summary of Available Data on the Stron tium-90 Content of Foods and Total Diets in the United States, U. S. AEC Report HASL-90 (August 1960).

5. J. H. Harley (Editor). EML Procedures Manual. U.S. DOE Report F\{ ASL-300 ( 1972, revised annually). 


\section{GENERAL REFERENCES}

\section{Radiochemical Methods}

National Academy of Sciences, Nuclear Science Series (NAS-NS 3001 ff), Office of Technical Services, Washington (1960 and later), A Series of Monographs on Individual Elements and Techniques.

WHO-FAO-IAEA, Methods of Radiochemical Analysis, World Health Organization, Geneva ( 1966).

Johns (Editor), National Environmental Research Center, Las Vegas, Handbook of Radiochemical Analytical Methods, EPA-680/4-75-001, February 1975.

Harley (Editor), Environmental Measurements Laboratory, DOE, EML Procedures Manual, HASL-300, 1972 (Updated Annually).

International Atomic Energy Agency, Reference Methods for Marine Radioactivity Studies (Sr, Cs, Ce, Co, Zn). Technical Reports Series No. 118 (1970).

Ibid. (Transuranics, Ru, Zr, Ag, I)

Technical Reports Series No. 169 (1975)

\section{Measurement of Radioactivity}

National Council on Radiation Protection and Measurements, A Manual of Radioactivity Measurements Procedures, Report 58 ( 1977).

National Council on Radiation Protection and Measurements, Environmental Radiation Measurements, Report 50 (1976).

International Commission on Radiation Units and Measurements, Measurement of Low-Level Radioactivity, Report 22 ( 1972).

International Atomic Energy Agency, Measurement of Short-Range Radiations, Technical Reports Series No. 150 ( 1973).
International Atomic Energy Agency, Metrology and Radionuclides (Symposium), IAEA, Vienna ( 1960).

Crouthamel (Editor), Revised by Adams and Dams, Applied Gamma-Ray Spectrometry, Pergamon, New York (1970).

\section{Data Compilations}

Lederer and Shirley, Table of Isotopes-7th Edition, John Wiley, New York (1978).

Health, Scintillation Spectrometry, Gamma-Ray Spectrum Catolog ( $3 \mathrm{rd}$ Edition), USAEC Report ANCR-1000 ( 1974).

Chanda and Deal, Catolog of Semiconductor Alpha-Particle Spectra, USAEC Report IN-1261, March 1970,

Niday and Gunnink, Computerized Quantitative Analysis of Gamma-Ray Spectrometry, USAEC Report UCRL-51061, 4 Volumes (Includes Decay Scheme Data Library).

\section{Quality Assurance}

Kanipe, Handbook for Analytical Quality Control in Radioanalytical Laboratories, EPA Report 600/777-088 (April 1977),

Ziegler and Hunt, Quality Control for Environmental Measurements using Gamma-Ray Spectrometry, EPA Report 600/7-77-144 (December 1977).

See also:

Metrology Needs in the Measurement of Environmental Radioactivity Environment International 1, $7 \mathrm{ff}$ (1978),

\section{GLOSSARY}

Activation Products-The radionuclides formed in either fissionable material or in surrounding material during a nuclear reaction, usually by capture of neutrons,

Carrier-A stable element added in radiochemical analysis to provide sufficient mass that a radionuclide can follow various separation steps.

Detector-Any device that transforms the radiation emitted by a radionuclide into a signal that can be handled electronically. The most usual signal is an electrical pulse which can be recorded.

Disintegration-The spontaneous process by which a radionuclide gives off energy in the form of nuclear radiation.

Fissionable Material-Any of the heavy radionuclides which can undergo fission, or splitting into two or more lighter atoms. The fission process is accompanied by a large release of energy.
Fission Products-The radionuclides formed when fissionable material splits into two or more atoms.

Geiger Counter-A detector based on gas ionization which converts any ionization within the detector into a large electrical pulse.

Germanium Diode Detector-A gamma-ray detector composed of very pure germanium, usually activated with lithium.

Indicator Food-A food that is measured to give an estimate of the total dietary intake of man.

Ionization Chamber-A radiation detector based on gas ionization which converts any ionization within the chamber into an equivalent electrical pulse.

Pathway-The route by which a radionuclide is transferred from the source through the environment to man.

Picocurie-See units of radioactivity. Equal to 2.2 disintegrations per minute. 
Proportional Counter-A detector based on gas ionization which converts any ionization within the detector into an amplified electrical pulse proportional to the amount of ionization.

Radionuclide-Any atomic species which is unstable and gives off nuclear radiation to attain stability.

Scintillation Detector-A detector which converts nuclear radiation to a pulse of light. This, in turn, can be converted to an electrical pulse with a phototube.

Sodium Iodide Detector-A scintillation gamma-ray detector made up of a single crystal of sodium iodide activated with silver.

Spectrometry-The measurement of the energy of radiation emitted during decay of a radionuclide or mixture of radionuclides. Quantitative as well as qualitative information may usually be derived.
Tracer-A radionuclide added in radiochemical analysis to follow the distribution of the desired constituent in various separation steps.

Transuranic Element-An artificial element having a higher atomic number than uranium, formed by activation, usually with neutrons.

Units of Radioactivity-There are various ways of expressing the disintegration rate of a radionuclide. In this report the disintegrations per minute (dpm) unit is used. In other reports, the curie and its submultiple (millicurie, microcurie, picocurie) are used and the new International Standard nomenclature is the bequerel, equal to one disintegration per second. 
1. Clement Associates, Inc., Washington, D. C., "Priority Setting of Toxic Substances for Guiding Monitoring Programs. "

2. Congressional Research Service, Science Policy Research Division,

- Christopher H. Dodge, "Contamination of Food by Radioactive Nuclides. '

- Sarah L. Hartman, "Nitrate Food Contamination: A Case Study. ",

- Jo-Ann M. McNally, "Mercury: Environmental Contamination of Food," "Polybrominated Biphenyls: Environmental Contamination of Food, "Polychlorinated Biphenyls: Environmental Contamination of Food.

3. Dr. William E. Cooper and R. V. Farace, Department of Zoology, Michigan State University, "Toxic Substances in Food Information System: Design and Management.",

4. Dr. Kenny S. Crump and Marjory D. Masterman, Science Research Systems. Ruston, La.,

*Available through the National Technical Information Service as "Environmental contaminants in Food. Volume IIWorking Papers." "Assessment of Carcinogenic Risks From
PCBS in Food."

5 Dr. Donald Epp, Department of Agricultural Economics and Rural Sociology, Pennsylvania State University, "Determining the Economic Impact of Alternative Action Levels."

6. Dr. Naomi H.Harlev, Institute of Environmental Sciences, New "York University Medical Center, "Analysis of Foods for Radioactivity.

7. Dr. John L. Laseter, Environmental Affairs, Inc., New Orleans, La. "Approaches to Monitoring Organic Environmental Contaminants in Food' (appendix G).

8. Dr. Frank Lu, School of Medicine, University of Miami, "Environmental Contaminants in Food: International Activities.

9. Dr. Rodney K. Skogerboe, Department of Chemistry, Colorado State University, "Analytical Systems for the Determination of Metals in Food and Water Supplies" (appendix H).

10. SRI International, Menlo Park, Calif., "Assessment of Methods for Regulating 'Unavoidable' Contaminants in the Food Supply.",

11. Tracer Jitco, Inc., Rockville, Md., "Case Studies on Consumer Risk from Environmental Contaminants in Food. " 


\title{
Executive Agency Observers at Advisory Panel Meetings
}

\author{
U.S. Environmental Protection Agency \\ Fred Arnold* Marty Halper* \\ Rufus Morrison Janice Ryan
}

U.S. Department of Health, Education, and Welfare

Food and Drug Administration

John Wessel* Charles Jelinek Pasquale Lombardo

Bart Puma Sidney Williams

U.S. Department of Agriculture

Food Safety and Quality Service

Grace Clark* Richard Ellis

William Leese John Spaulding

*Official agency liaison to the assessment, 